\title{
CENTRIFUGAL SCALING OF ISOTHERMAL GAS-LIOUID FLOW IN HORIZONTAL TUBES
}
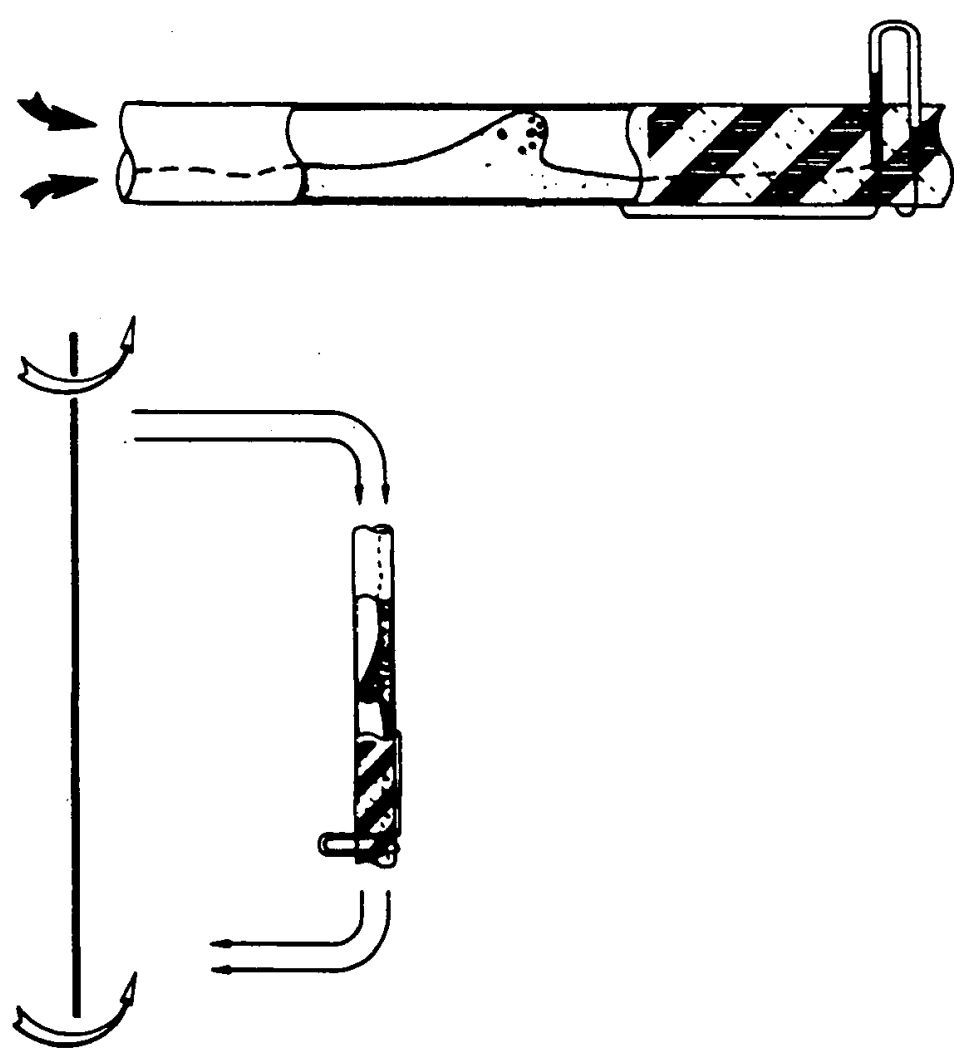

4 TR diss 
$33 \geq 162$

310231

ph diss is wo

CENTRIFUGAL SCALING OF ISOTHERMAL GAS-LIOUID FLOW IN HORIZONTAL TUBES 


\title{
CENTRIFUGAL SCALING OF ISOTHERMAL GAS-LIQUID FLOW IN HORIZONTAL TUBES
}

\author{
PROEFSCHRIFT \\ ter verkrijging van de graad van doctor \\ in de technische wetenschappen aan de \\ Technische Universiteit Delft, op gezag van \\ de Rector Magnificus, prof.dr. J.M. Dirken, \\ in het openbaar te verdedigen \\ ten overstaan van het College van Dekanen \\ op 9 oktober 1986 te 14.00 uur \\ door \\ JACQUES JOSEPH MARIE GERAETS \\ geboren te Neer \\ natuurkundig ingenieur
}

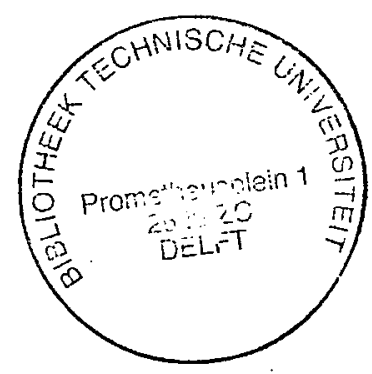


Dit proefschrift is goedgekeurd door de promotor prof.dr.ir. G. Ooms 
aan Nelly en Wim 


\section{SUMMARY}

1 INTRODUCTION

1.1 General introduction

1.2 Purpose of the investigation

1.3 Flow regimes in horizontal two-phase flow

1.4 Pressure drop and vold fraction in two-phase flow

1.5 Scaling in two-phase flow

2 SCALING OF ISOTHERMAL TWO-PHASE FLOW 9

2.1 Modelling laws from dimensional analysis 9

2.2 Similarity criteria from the conventional equations 10

2.2.1 The conventional equations 10

2.2.2 The similarity criteria 13

2.2.3 Validity of the conventional equations 16

$\begin{array}{lll}2.3 & \text { Satisfaction of the criteria } & 17\end{array}$

2.3.1 Constant gravity 18

2.3.2 8 variable: rotating model 18

2.3.3 Consequences of the use of rotation 20

2.4 Measurement programme 22

2.4.1 The scaling test A 23

2.4.2 Variation of the dimensionless groups 26

2.4 .3 The scaling test B 27

2.5 Conclusions 30

3 THE SENSOR FOR VOID FRACTION MEASUREMENT

AND FLOW PATTERN IDENTIFICATION 31

3.1 Background of the impedance method 31

3.2 Construction of the sensor 33

3.3 The capacitance of the helical cross-capacitor 36

3.4 Calibration curves 41

3.4.1 Annular flow 41

3.4.2 Dispersed flow 45

3.4.3 Stratified flow 47

3.4.4 Measurement strategy for intermittent flow 49

3.5 Flow pattern identification from void fluctuations 51

3.6 Conclusions 53 
THE TEST FACILITY FOR TWO-PHASE EXPERIMENTS

AT INCREASED GRAVITY

4.1 The centrifuge facility 54

$\begin{array}{lll}4.1 .1 & \text { The rotor } & 54\end{array}$

4.1.2 Critical speed and balancing of the rotor 58

4.1.3 The drive and bearings 60

4.1.4 The seals $\quad 62$

4.1.5 The slip-rings 63

4.2 The rotating test circuit 65

4.2.1 Description of the circuit and test section 65

4.2.2 Filling of the circuit 68

4.3 Measurement of pressure drop $\quad 69$

4.4, Adjustment of main parameters 75

4.4.1 The liquid circuit $\quad 76$

4.4.2 The gas circuit 76

4.4.3 General procedure for measurement $\quad 77$

4.4.4 Accuracy of main parameters and dimensionless groups 80

$\begin{array}{lll}4.5 & \text { Conclusions } & 84\end{array}$

5 THE 50 AND 100 MM TEST FACILITIES $\quad 85$

5.1 Description of $50 \mathrm{~mm}$ test facility 85

5.1.1 The test section $\quad 85$

5.1 .2 Measurement of pressure drop $\quad 87$

5.1.3 Supply of liquid and gas 88

5.1.4 General procedure for measurements 90

5.2 Preliminary experiments 91

5.2.1 Influence of the separator 91

5.2.2 Influence of the inclination angle 92

5.2.3 Flow development 93

$\begin{array}{lll}5.2 .4 & \text { Conclusions } & 94\end{array}$

5.3 The $100 \mathrm{~mm}$ test facility 95

5.3.1 Description of the loop 95

$\begin{array}{lll}5.3 .2 & \text { Measurement of pressure drop } & 97\end{array}$

5.3.3 Measurement of void fraction 97

5.3.4 Data aquisition and reproducibility 99

5.3.5 Experimental: influence of gas inlet geometry 100

$\begin{array}{llr}5.3 .6 & \text { Conclusions } & 103\end{array}$ 
6 EXPERIMENTAL RESULTS: CENTRIFUGAL SCALING 104

6.1 Length scale factor of 0.1 : the scaling test A 104

6.1.1 Comparison of the pressure drop 104

6.1.2 Comparison of the void fraction 106

6.1.3 Comparison of the flow pattern 106

6.1 .4 Conclusions 112

$\begin{array}{lll}6.2 & \text { Comparison with literature } & 113\end{array}$

6.3 Influence of the dimensionless groups $\quad 117$

6.3.1 Influence of the Euler number 117

6.3.2 Influence of the density ratio 118

6.3.3 Influence of the Reynolds number 119

$\begin{array}{ll}6.3 .4 & \text { Influence of the Froude number } \\ 6.320\end{array}$

6.3.5 Influence of the Weber number 124

6.3.6 Conclusions 126

6.4 Length scale factor of 0.05 and 0.5 : the scaling test $B \quad 127$

$\begin{array}{lll}6.4 .1 & \text { Comparison of the pressure drop } & 127\end{array}$

6.4.2 Comparison of the void fraction 129

6.4.3 Comparison of the flow pattern 130

$\begin{array}{lll}6.4 .4 & \text { Conclusions } & 133\end{array}$

$7 \quad$ APPLICATIONS OF CENTRIFUGAL SCALING 134

8 FINAL CONCLUSIONS 137

Appendix I Test conditions and experimental results of the scaling test A 139

Appendix II Test conditions and experimental results of the scaling test B 146

Appendix III Capacitance measurements with conducting media 150

$\begin{array}{lr}\text { LIST OF PRINCIPAL SYMBOLS } & 153\end{array}$

$\begin{array}{ll}\text { LIST OF REFERENCES } & 156\end{array}$

$\begin{array}{ll}\text { Samenvatting } & 163\end{array}$

$\begin{array}{ll}\text { Nawoord } & 165\end{array}$

$\begin{array}{ll}\text { Curriculum vitae } & 166\end{array}$ 
For testing the similarity criteria of two-phase gas-liquid flows, arising from the governing equations and boundary conditions, the flow of air and a water-glycerine mixture in a $50 \mathrm{~mm}$ diameter horizontal tube is compared with a two-phase flow of helium and water in a $5 \mathrm{~mm}$ diameter tube rotating around a parallel vertical axis (the effective gravity is $113 \mathrm{~g}_{0}$ ). A general test facility has been built, enabling unique measurements of pressure drop, void fraction and flow pattern at centrifugal accelerations up to 1200 times natural gravity.

The Reynolds number, Froude number, Weber number and gas-liquid density ratio are equal in the 5 and $50 \mathrm{~mm}$ flows. Compressibility and gas-viscosity effects are not scaled. Much attention is paid to the geometrical similarity of the two test sections: the same length to diameter ratio, the same gas inlet geometry and the same outlet conditions are applied. In behalf of the scaling tests a capacitive sensor for measuring void fraction and flow pattern (defined from the time-varying void signal) is developed. Because the same type of sensor is used for the 5 and $50 \mathrm{~mm}$ test sections very accurate comparisons of these quantities are possible.

With a few exceptions the agreement between the two "different" flows is excellent. The dimensionless pressure drops agree within $19 \%$ and the absolute differences of the void fraction are less than 0.03 . In seventeen out of eighteen examined flow situations the flow pattern was the same at the two scales. The frequency scale factor, determined from the measured plug and slug frequencles agrees extremely well with the theoretical value. At small liquid and gas flow rates larger differences are observed, probably due to specific loop-dependent conditions such as the uncertainty in the position of the test section with respect to the rotation axis, back flow of liquid from the separator and vibrations of the system.

The influence of the individual dimensionless groups has been investigated, the Froude number in particular being readily varied in a rotating system. It appears that this group and the gas-liquid density ratio are the most important parameters. However at high gas velocities an increase of the Weber number by a factor of 2 causes a 23\% decrease of the pressure drop. The influence of the Euler number and gas-liquid dynamic viscosity ratio is minor in the flow regimes investigated. 
Comparisons are presented with general pressure drop and void fraction correlations. The results emphasize that in general only dimensionless correlations will provide meaningful predictions. The homogeneous Dukler case I (1964b) correlation, which contains no two-phase information provides the best $f$ it (the standard deviation is $21 \%$ ) with the measured pressure drops. For predicting flow pattern the most promising approach is that of Taitel and Dukler (1976).

Finally some examples of scaling down large diameter, high pressure pipelines are presented. With a scale factor of $1 / 30$ equality of the Froude number, the gas-liquid density ratio and either the Reynolds number or the Weber number can be realized. Compressibility and gas viscosity are not properly scaled in general. 


\section{$I$ INTRODUCTION}

\subsection{GENERAL INTRODUCTION}

The prediction of pressure drop, vold fraction and flow pattern during the simultaneous flow of liquid and gas or vapour is necessary for economic design and optimization of operating conditions in the petroleum, power and chemical industry. In a gas-oll line for instance the mixture is transported at high pressures over long distances and for calculating the plpe diameter or the dimensions of the liquid gas separator it is important to know the pressure drop and vold fraction. The design of water tube bollers, refrigeration equipment, water cooled reactors, evaporators and many other major items of chemical and power plant is dependent upon a knowledge of the fluid dynamics and heat transfer processes. The conditions under which the mixtures flow are very divergent: pipe diameters from $1 \mathrm{~mm}$ up to $1 \mathrm{~m}$, pressures from $20 \mathrm{MPa}$ to atmospheric, horizontal and vertical $\mathrm{flow}$, concurrent and countercurrent flow.

While similarity criteria for flows of single-phase fluids have been well established and modelling using these criteria has long been an accepted practice, such determination for two-phase mixtures has been hampered by conflicting and incomplete formulations of the basic equations. However if only relatively simple two-phase flows are considered, 1.e. isothermal flow without mass transfer, similarity criteria can be developed in a rigorous way from the governing equations and boundary conditions (Chesters, 1975). In 1 tself the similarity criteria do not provide a relation for predicting pressure drop or flow pattern. However once the relationship is found (analytically or from experimental data) for one system, the conditions of dynamic similarity require the same relationship to apply to all similar systems.

Until now no comprehensive experimental study has been carried out on the applicability of similarity criteria for scaling down horizontal two-phase plpeflow. Chesters (1977) showed that a conslderable length scale down factor can only be realized if the gravitational force in model is several hundred times earth gravity. The latter can be achieved by rotating the tube with the two-phase flow around a parallel vertical axis. The direction of the centrifugal force is normal to the tube axis, which restricts the method to horizontal or nearly horizontal flow. 


\subsection{PURPOSE OF THE INVESTIGATION}

The aims of the present study are:

- 1 - to develop an instrument for scaling down two-phase flows in horizontal or nearly horizontal tubes.

- 2 - to test the applicability of the similarity criteria which are derived from the governing equations and boundary conditions.

- 3 - to examine the influence of the various dimensionless group separately e.g. the Froude number can easily be varied in a rotating test section, while keeping the other similarity parameters constant. This is not the case in static systems.

A centrifuge facility has been built which enables unique measurements of pressure drop, vold fraction and flow pattern in gas-liquid flows at gravities up to 1200 times natural gravity. For testing the similarity criteria two series of experiments have been carried out. The scaling test $A$ includes very accurate two-phase measurements for a length scale factor of 0.1 . The pressure drop, void fraction and flow pattern in a $50 \mathrm{~mm}$ stationary test section are compared with those measured in a $5 \mathrm{~mm}$ rotating test section. The system pressure, temperature and rotation rate are chosen in such a way that dimensionless groups can be varied relatively easy. Much attention is paid to the geometrical similarity of the two test sections.

For the scaling test $B$ besides the 5 and $50 \mathrm{~mm}$ test section, also a stationary $100 \mathrm{~mm}$ test section is used, allowing a length scale factor of 0.05 and 0.5 .

In chapter 2 the similarity criteria for isothermal gas-liquid flow without mass transfer are given. The capacitive sensor for measuring void fraction in the $50 \mathrm{~mm}$ and $5 \mathrm{~mm}$ test section is described in chapter 3 . The chapters 4 and 5 deal with the experimental set-ups for the rotating and stationary test sections. Moreover some experimental results on the influence of the separator, the mixing section and the downward inclination of the test section on the two-phase measurements are given. The comparison of the experimental results of the three test sections and the influence of various dimensionless groups are presented in chapter 6. In chapter 7 some examples of scaling down practical flows will be considered and the possibilities of the present facility are discussed. 


\subsection{FLOW REGIMES IN HORIZONTAL TWO-PHASE FLOW}

The most important characteristic of two-phase flow is the presence of interfaces separating the phases. Depending on the geometry of the interfaces, the flow of the two-phase mixture can be classified into several flow regimes. Flow regimes in horizontal flow tend to be somewhat more complex than those in vertical flow. This results from the asymetry in the flow induced by the gravitational force acting normal to the mean direction of the flow, which makes the heavier phase tend to accumulate at the bottom of the channel.

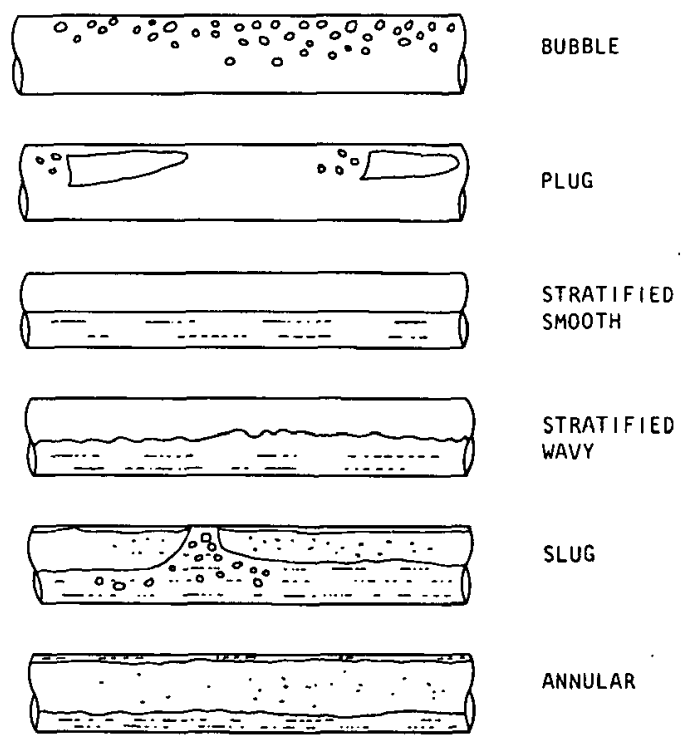

\section{Figure 1.1: Flow regimes in horizontal flow.}

A wide variety of flow pattern definitions have been proposed for horizontal flow. The following classification is often used, see fig. 1.1. - Bubble flow - Here, the bubbles are more or less dispersed in a liquid continuum. The bubbles tend to congregate near the top of the tube. At high liquid velocities, the bubbles may be more uniformly distributed. This flow 
pattern could not be established because of the limited capacity of the pumps.

- Stratified flow - The gravitational force separates the liquid and gas. At high gas velocities small waves are present at the liquid surface and the flow is called stratified wavy in contrast with stratified smooth.

- Intermittent flow - A variety of complex intermittent flows can exist in horizontal tubes and it is of ten appropriate to treat all such flows as being of a single generic type of flow regime. However the class of intermittent flow is often divided into two regions. The subdivision is very subjective and no general stringent criteria are available. An often used subdivision is:

- Plug flow - A kind of bullet shaped bubbles flow along the top of the pipe.

- Slug flow - This type of flow is characterised by passages of frothy slugs in which the liquid phase is continuous. Gas bubbles can be entrained in the liquid slug.

- Annular flow - The liquid flows on the wall of the tube as a film and the gas flows in the centre. (The film at the bottom of the tube is much thicker). A great number of small liquid droplets are entrained in the gas core. If the film at the top of the tube is very thin compared to the one at the bottom, here the flow is called semi-annular.

In this work beside visual observations the time-varying signal of a capacitive sensor for vold fraction measurements is used for determining the flow pattern. Because the active length of the sensor is six diameters, a somewhat different subdivision between plug and slug flow is used. If the distance between two bullet shaped bubbles is more than six diameter, what can easily be seen from the output signal, then the flow is called plug otherwise slug.

Reliable prediction of the flow regime for concurrent gas-liquid flow is still an unresolved problem in two-phase flow. The usual approach is to collect data for flow rates and fluid properties and to visually observe the flow pattern trough a transparent test section. Then a search is undertaken for a way to map the data in a two-dimensional plot by locating transition boundaries between the regimes. A major difficulty that arises is that flow pattern designations based on optical or visual observations depend much on individual interpretations. Much attention is paid to relate flow regime 
definitions to instrumental indications (Delhaye and Cognet 1984, v.d. Geld 1985. Jones and zuber 1975). However instrumental definitions first have to be related to the definitions extracted from visiual observations and the application of this method has not been wide spread. Presenting the flow regimes in a two-dimensional plot requires a decision to be made about the co-ordinates. Since, however, the flow regime is governed by at least six dimensionless groups a two-dimensional plot is quite inadequate for general representation.

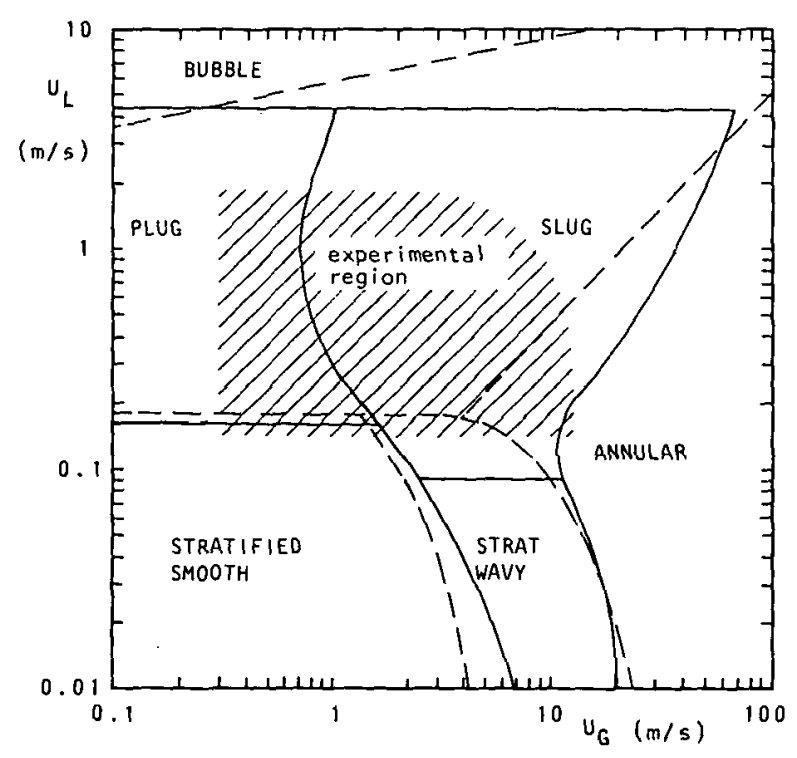

Figure 1.2: Flow pattern map for horizontal flow, Da50 mm, - Mandhane (1974), -- Taitel/Dukler (1976).

One of the best known flow pattern maps is that of Baker (1954). Despite 1ts dimensional nature, it is still used, mostly in the modified form of Bell (1969) or scott (1963). There have been a number of attempts at a theoretical or semi-theoretical description of flow regime transitions in order to be sultable for extrapolation to a wide range of conditions. A comprehensive treatment of plow regime tansitions on a semi-theoretical basis was presented by Taitel and Dukler (1976). The varlous transitions are 
expressed in terms of three dimensionless groups which are uniquely related to the independent variables of the systems. The transitional values of these groups are calculated in terms of the Lockhart-Martinelli parameter $x^{2}$, which is the ratio of the single-phase liquid to single-phase gas pressure drop. In figure 1.2 the Taitel Dukler prediction for an atmospheric air-water flow in a $50 \mathrm{~mm}$ dlameter pipe has been compared with the empirical map of Mandhane (1974) for atmospherlc air-water flow. Mandhane suggested a dimensional correlation of regime boundaries in terms of only the superficial velocities of the phases. In figure 2.1 the experimental region of the present investigation is indicated by the hatched area.

\section{Flow regimes in inclined tubes}

Most experiments in this study are carried out at small downward inclinations ( $<0.5$ degree) of the test section. Barnea e.a. (1980) have reported data for flow pattern transitions of atmospheric alr-water flow in a $25 \mathrm{~mm}$ diameter tube with small inclinations. The most remarkable feature is the great sensitivity of the position of the stratified to intermittent transition to the angle of inclination. With a downward inclination there is a preponderance of the stratified regime, and with upward inclination a preference of the intermittent regime. The influence of a small downward inclination on the flow pattern and pressure drop has also been investigated in the present work, see section 5.2.2.

\subsection{PRESSURE DROP AND VOID FRACTION IN TWO-PHASE FLOW}

The calculation of two-phase pressure drop and void fraction is an essential issue in many industrial applications and quite a number of studies and survey papers have been devoted to this area. For the determination of the frictional pressure drop, one must recall that this quantity is not usually measured directly. Most experimental methods provide values of the total pressure drop only. In general vold fraction measurements are needed to determine the acceleration and gravitational pressure drops. Even with the small downward inclinations $(<0.5$ degree $)$ used in this investigation the gravitational pressure drop can be a considerable part of the total pressure drop. An accurate measurement of the void fraction is therefore needed to enable determination of the frictional pressure drop, see chapter 3 . In 
1. Karakterisering van een twee-fasen stroming dient met behulp van dimensieloze getallen te geschieden. Grootheden met dimensie geven meer informatie over de gebruikte apparatuur en infrastructuur dan over de ingestelde stroming.

2. Door een ongelukkige keuze van de wanddikte van de perspex binnenmantel kan met de capacitleve sensoren van Gregory (1973) en Abouelwafa (1979) slechts stromingspatroon-onafhankelijke bepalingen van de gasvolumefractie uitgevoerd worden als de permittiviteit van het gas en de vloeistof weinig verschillen.

3. Hysterese effecten treden nagenoeg niet op bij twee-fasen stromingen van Newtonse fluida.

4. De invloed van oppervlakte actieve stoffen in praktische twee-fasen stromingen kan dusdanig groot zijn dat het Weber getal geen relevante parameter meer is.

5. De interactie van een eenheidsverstoring (bv. een zogenaamde "hairpin vortex") met een enkel stofdeeltje in een laminair stromingsveld dient de basis te zijn voor de ontwikkeling van modellen ten behoeve van de beschrijving van stoftransport in een turbulente grenslaag.

6. De regelingen voor indiensttreding en afvloeling bij het voortgezet onderwijs verlagen in niet onbelangrijke mate de mobiliteit van het onderwijspersoneel.

7. Het nuttig georuik van de computer in hulshoudens beperkt zich voorlopig tot activiteiten in de hobbysfeer.

8. Verlaging van het Froude getal bij horizontale twee-fasen stroming leidt, in tegenstelling tot hetgeen voorspeld wordt door de Friedel correlatie (1979), tot lagere drukvallen.

9. Problemen bij het opschalen van het electrostatisch copieerproces van A4-formaat naar A0-format komen grotendeels voort uit het niet consequent (kunnen) toepassen van de schaalfactor voor de geometrie.

10. Met behulp van zogenaamde "cellular automata" kunnen stromingsproblemen in de toekomst wellicht op een goedkopere en eenvoudigere manier opgelost worden dan met de bestaande numerieke technieken.

Frisch U., Hasslacher B. en Pomeau $Y$. Phys. Rev. 56, pp 1505-1508, 1986

11. Omdat "instrumentele definities" van stromingspatronen in veel gevallen gekoppeld zijn aan subjectieve visuele warnemingen is een redelijke mate van objectiviteit pas dan gewaarborgd als algemeen geldende afspraken omtrent de indeling van stromingspatronen gemaakt worden aan de hand van bijvoorbeeld fotografische waarnemingen. 
two-component two-phase flow the accelaration pressure drop, due to the expansion of the gas can usually be neglected.

A great number of empirical correlations and corrections to existing correlations which are often used have been presented in literature. Many of them can be found in comparative studies of Mandhane et al. (1977) and Dukler et al. (1964). With a few exeptions they have the common fault of lacking generality. Each correlation reproduces the data used for its development rather well but is often grossly incorrect beyond this domain. Even the best "general" correlations for two-phase pressure drop are capable of predicting only the level. Standard deviations of $40 \%$ are encountered. The correlations which have demonstrated the greatest success have been those based on a simplified model of the complex two-phase system.

In chapter 6 some of the best-known correlations (Lockhart-Martinelli 1949, Dukler et al. 1964, Friedel 1979, Chrisholm 1973) will be compared with the results of the scaling tests.

\subsection{SCALING IN TWO-PHASE FLOW}

The reason for the use of similarity criteria in two-phase flow arises from the considerations of cost and time. It also provides a better understanding of how to deal with two-phase flow and it assits in the comparison and generalisation of data.

In the petroleum industry an important question is how to translate small-scale laboratory tests into large-scale industrial plant performance. Often only two dimensionless groups are used: the Froude number and the gas liquid density ratio. In the power industry scaling of the fluid, rather than that of the physical dimensions is of interest, in order to obtain low heat fluxes, pressures and temperatures in model work (Mayinger, 1977). High pressure steam-water systems are scaled by freon systems at much favourable conditions on a 1:1 length scale. To obtain a reasonable agreement between prototype and model correction factors and ratios of dimensionless groups are often used in a somewhat arbitrary way (Friedel 1974). 
In general there are several methods avallable for developing scaling laws for a particular system of interest. These may be classified as follows:

- Method based on Buckingham's Theorem (1914). When the parameters involved in the phenomena are known or can be guessed, while the physical laws governing the variables are not known, Buckingham's Theorem can be used to obtain dimensionless parameters. Many of the conventional two-phase flow studies on pressure drop, bolling heat transfer and critical heat flux have been obtained in this manner.

- Methods based on physical laws. When the physical laws governing a phenomena are well understood and the equations describing them are available, these equations can be made dimensionless with the aid of scales characteristic of various physical parameters involved in the system. From these equations similarity groups, having clear physlcal significance can be obtained. This method is useful for examining the relative importance of various physical effects and mechanisms operating in the system. The usefulness of the similarity parameters obtained, often depends critically on the choice of characteristic scales.

Any exact or approximate solution of the governing equations can also be expressed in dimensionless variables with the help of characteristic scales, leading to the same similarity parameters as when this process is applied to the original equations. 


\section{SCALING OF ISOTHERMAL TWO-PHASE FLOW}

Modelling laws can be derived by either non-dimensionalizing the governing equations and their boundary and initial conditions or by using the method of dimensional analysis, starting only with a knowledge of the physical quantities which specify the problem. The first method is outlined in section 2.2, the second one is given below. In section 2.4 the scale factors and experimental conditions for testing the similarity criteria are given.

\subsection{MODELLING LAWS FROM DIMENSIONAL ANALYSIS}

Consider two systems with a two-phase flow of a gas and a liquid, having the same dimensionless initial and boundary conditions at all dimensionless times. For a horizontal tube for instance the two requirements imply that the systems must be geometrical similar and the same ratio of the gas and liquid volume flow rate must be applied. The requirement of "geometrical similarity" includes: the same length to diameter ratio, the same wall roughness to diameter ratio, the same inlet and outlet geometry etc. For comparing (=scaling) the flow pattern, the dimensionless pressure drop, the vold eraction or any other dependent dimensionless quantity of the two systems, the following nine parameters are of interest: the density $\rho_{L}$ and dynamic viscosity $\mu_{L}$ of the liquid, the density $\rho_{G}$ and dynamic viscosity $\mu_{G}$ of the gas, the surface tension $\sigma$, the system pressure $p$, the acceleration due to gravity 8 , a characteristic length $L$ of the system and a characteristic velocity $U$ of the flow. Only three of the nine independent physical parameters are dimensionally independent. It follows from dimensional analysis (Buckingham 1914) that six independent dimensionless quantities (similarity parameters) uniquely specify the problem.

Let $\rho_{L}, U$ and $L$ be the three dimensionally independent parameters, then the six independent similarity parameters can be found by replacing the physical constants by their dimensionless equivalents:

$$
\begin{array}{ll}
\mu_{L} \rightarrow \frac{\mu_{L}}{\rho_{L} U L}=\frac{1}{R e} & \text { Re }=11 \text { quid Reynolds number } \\
\rho_{G} \rightarrow \frac{\rho_{G}}{\rho_{L}} & \text { density ratio }
\end{array}
$$




$$
\begin{array}{ll}
\mu_{G} \rightarrow \frac{\mu_{G}}{\rho_{L} U L}=\frac{\mu_{G}}{\mu_{L}} \frac{1}{R e} & \text { viscosity ratio } \\
\sigma \rightarrow \frac{\sigma}{\rho U^{2}}=\frac{1}{W e} & \text { We }=\text { Weber number } \\
g \rightarrow \frac{g L}{U^{2}}=\frac{1}{F r} & \text { Fr }=\text { Froude number } \\
p \rightarrow \frac{p}{\rho_{L} U^{2}}=E u & \text { Eu }=\text { Euler number }
\end{array}
$$

Summarizing: if the six dimensionless numbers are equal for two geometrically similar systems then any dependent varlable, made dimensionless with respect to the three independent characteristic quantities must be equal for both flows. In this way the six dimensionless groups can be regarded as the recipe for carrying out scaling experiments for two-phase flows. The similarity criteria can be derived in a rigorous way from the conventional equations (Chesters, 1978), see section 2.2.

\subsection{SIMILARITY CRITERIA FROM THE CONVENTIONAL EQUATIONS}

\subsubsection{The conventional equations}

The situation to be described is that of discrete zones of each fluid bounded by a solid surface or by the other fluid (see figure 2.1).

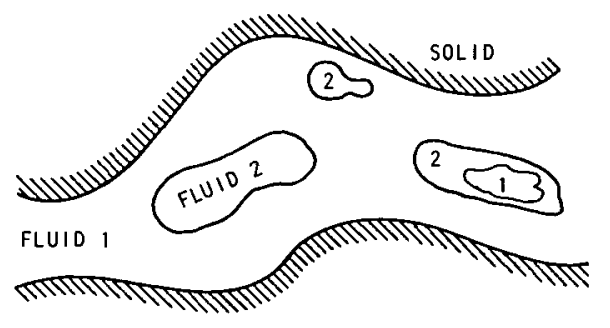

Figure 2.1: General gas-Iiquid flow. 
Under "normal" conditions (see section 2.2.3) the flow of each phase is governed by the conventional equations, that is: the continuum equations (Navier-Stokes, energy, continuity, state), the interface and solid-boundary conditions appropriate to an abrupt discontinuity in phase, and the initial conditions. For Isothermal flows, the local thermodynamic state of each fluid is determined by the extra information provided by its temperature, without recourse to the energy equation which thus becomes superfluous. Neglecting liquid compressibility, the equations to be satisfied within each phase are therefore:

\section{The gas}

$$
\begin{array}{ll}
\text { Navier-stokes } & \frac{D \underline{u}_{G}}{D t}=g \underline{k}-\frac{1}{\rho_{G}} \nabla p_{G}+\frac{\mu_{G}}{\rho_{G}}\left[\frac{1}{3} \nabla\left(\nabla \cdot \underline{u}_{G}\right)+\nabla^{2} \underline{u}_{G}\right] \\
\text { Continuity } & \frac{D \rho_{G}}{D t}=-\rho_{G}\left(\nabla \cdot \underline{u}_{G}\right) \\
\text { State } & p_{G}^{\prime \rho} \rho_{G}=\text { constant }
\end{array}
$$

The liquid

$$
\begin{array}{ll}
\text { Navier-Stokes } & \frac{D \underline{u}_{L}}{D t}=g \underline{k}-\frac{1}{\rho_{L}} \nabla p_{L}+\frac{\mu_{L}}{\rho_{L}} \nabla^{2} \underline{u}_{L} \\
\text { Continuity } & \nabla \cdot \underline{u}_{L}=0 \\
\text { State } & \rho_{L}=\text { constant }
\end{array}
$$

where $\underline{u}, p, \rho, \mu, g$ and $t$ denote respectively velocity, pressure, density, dynamic viscosity, acceleration due to gravity and $t i m e, k$ is a unit vector in the direction of $B$, and the subscripts $G$ and $L$ denote gas and 11 quid.

At the gas-liquid interface the conventional conditions to be satisfied are: Continuity of velocity (1.e. no slip):

$$
\underline{u}_{G}=\underline{u}_{L}
$$




$$
p_{G}{ }^{\prime} / \rho_{G}{ }^{\prime}=\text { constant }
$$

$$
\frac{D \underline{u}_{L}^{\prime}}{D t^{\prime}}=\left(\frac{g L}{U^{2}}\right) \underline{k}-\nabla^{\prime} p_{L}^{\prime}+\left(\frac{\mu_{L}}{\rho_{L} U L}\right) \nabla^{\prime 2} \underline{u}_{L}^{\prime}
$$

$$
\nabla^{\prime} \underline{u}_{L}^{\prime}=0
$$

$$
\rho_{L}^{\prime}=1
$$

$\underline{u}_{G}^{\prime}=\underline{u}_{L}^{\prime}$

$\left(\frac{{ }_{G}}{\rho_{L} U_{L}}\right)\left(\frac{\partial u_{3}^{\prime}}{\partial x_{1}^{\prime}}+\frac{\partial u_{1}^{\prime}}{\partial x_{3}^{\prime}}\right)_{G}=\left(\frac{\mu_{L}}{\rho_{L} U L}\right)\left(\frac{\partial u_{3}^{\prime}}{\partial x_{1}^{\prime}}+\frac{\partial u_{1}^{\prime}}{\partial x_{3}^{\prime}}\right)_{L}$

$\left(\frac{{ }_{G}}{\rho_{L} U^{\prime}}\right)\left(\frac{\partial u_{3}^{\prime}}{\partial x_{2}^{\prime}}+\frac{\partial u_{2}^{\prime}}{\partial x_{3}^{\prime}}\right)_{G}=\left(\frac{\mu_{L}}{\rho_{L} U_{L}}\right)\left(\frac{\partial u_{3}^{\prime}}{\partial x_{2}^{\prime}}+\frac{\partial u_{2}^{\prime}}{\partial x_{3}^{\prime}}\right)_{L}$

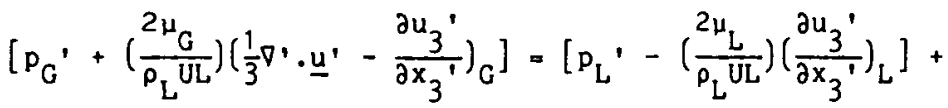

$\left(\frac{\sigma}{\rho_{L} U^{2}}\right)\left(\frac{1}{R_{1}^{\prime}}+\frac{1}{R_{2}}\right)$

$\underline{u}_{G}^{\prime}$ or $L=\underline{u}_{\text {Sol }}^{\prime}$

where $\nabla^{\prime}$ denotes the vector operator with components $\frac{\partial}{\partial x_{1}{ }^{\prime}}, \frac{\partial}{\partial x_{2}{ }^{\prime}}$ and $\frac{\partial}{\partial x_{3}{ }^{\prime}}$.

\subsubsection{The similarity criteria}

The dimensionless governing equations (2.11) to (2.20) are seen to be identical in two flows provided five dimensionless parameters are equal:

$$
\begin{array}{ll}
\text { Re }=\rho_{L} U L / \mu_{L}=\text { equal } & \text { Reynol ds number } \\
W e=\rho_{L} U^{2} / \sigma=\text { equal } & \text { Weber number } \\
\mathrm{Fr}=U^{2} / g L=\text { equal } & \text { Froude number } \\
E=p_{G} / \rho_{G} U^{2}=\text { equal } & \text { Euler number } \\
\mu_{G} / \rho_{G} U L=\text { equal } & \text { (or } \mu_{G} / \mu_{L} \text { in view of 2.21) }
\end{array}
$$


If, furthermore, the initial and boundary conditions of the flow variables $\underline{u}_{L}{ }^{\prime}, \underline{u}_{G}{ }^{\prime}, p^{\prime}$ and $\rho_{G}{ }^{\prime}$ are equal, the two flows, in so far as they are governed by the conventional equations, will be dynamicaliy similar. That is, $\underline{u}_{L}{ }^{\prime}, \underline{u}_{G}{ }^{\prime}, p^{\prime}$, and $\rho_{G}^{\prime}$ will have equal values at corresponding points at all (dimensionless) times. Equality of $\underline{u}_{G}{ }^{\prime}$, and $\underline{u}_{L}{ }^{\prime}$ is normally expressed as equality of flow pattern and includes not only global features but all details of the flow: the relative scale and intensity of turbulence, the relative size of droplets, etc.

Equality of initial and boundary conditions on $\underline{-}_{L}{ }^{\prime}, \underline{u}_{G}{ }^{\prime}, p^{\prime}$ and $\rho_{G}{ }^{\prime}$ requires that imposed distributions of these variables be geometrically similar. Thus, for example inlet velocity profiles must have the same shape and any solid boundary surfaces must, in view of (2.20), be geometrically similar (at all dimensionless times). Naturally, the absolute imposed values of $\underline{u}_{L}{ }^{\prime}, \underline{u}_{G}{ }^{\prime}, p^{\prime}$ and $\rho_{G}{ }^{\prime}$ must be equal in some characteristic point of the flow (at all dimensionless times):

$$
\begin{aligned}
& \left(\underline{u}_{L}^{\prime}, \text { char }\right)_{1}=\left(\underline{u}_{L}^{\prime}, \text { char }\right)_{2} \\
& \left(\underline{u}_{G, \text { char }}^{\prime}\right)_{1}=\left(\underline{u}_{-G, \text { char }}^{\prime}\right)_{2} \\
& \left(p_{\text {char }}^{\prime}\right)_{1}=\left(\rho_{\text {char }}^{\prime}\right)_{2} \\
& \left(\rho_{G, \text { char }}^{\prime}\right)_{1}=\left(\rho_{G}^{\prime}, \text { char }\right)_{2}
\end{aligned}
$$

In cases where a liquid velocity is imposed (2.26) is most easily satisfied by the choice $U=u_{L,}$ char in which case $u_{L, \text { char }}=1$ in both flows. Requirement (2.27) then becomes equivalent to the requirement of equal velocity ratio of the two phases. Here for $u_{L, \text { char }}$ is chosen to be the superficial liquid velocity $U_{L}$, which is the mean liquid velocity in the tube before gas is added to the flow.

In view of the requirement of equal Euler number $\left(p_{G} / p_{G} U^{2}\right)$ in both flows, requirement $(2.28)$, equal $\mathrm{p} / \mathrm{\rho}_{\mathrm{L}} \mathrm{U}^{2}$ in any characteristic point (here the gas outlet of the separator is chosen) demands that:

$$
\frac{\rho_{G, \text { char }}}{\rho_{L}}=\text { equal } \quad \text { or } \quad\left(\rho_{G, \text { char }}^{\prime}\right)_{1}=\left(\rho_{G, \text { char }}^{\prime}\right)_{2}
$$

The last requirement, (2.29), is thus automatically satisfied. 
Summarizing: besides equality of any imposed distributions of velocity and pressure and of the ratio of gas to liquid velocities, dynamic similarity of the two flows requires equality of the six dimensionless parameters Re, We, Fr, Eu, $\mu_{G} / \mu_{L}$ and ${ }^{\rho_{G}}$, char ${ }^{\prime} \rho_{L}$. The first five parameters arise from the conventional governing equations, the last one from the boundary conditions.

Any one of the six parameters may of course be replaced by any combination of itself with the others. The Euler number defined by (2.24) is replaced by a new Euler number, denoted by Eu:

$$
E u=\frac{p_{G, \text { char }}}{\rho_{L} U^{2}}=E \frac{\rho_{G, \text { char }}}{\rho_{L}}
$$

The new Euler number is of the order of the ratio of the system pressure and the two-phase pressure drop over 40 diameters ( $=$ distance between the pressure taps). From the first three parameters Reynolds, Weber and Froude the scale factors for length, velocity and viscosity can be derived:

We/Fr leads to

$$
\frac{L^{2} \rho_{L}^{g}}{\sigma}=\text { equal } \quad(2.32) \quad \text { or } \quad \frac{L_{2}}{L_{1}}=\frac{\left(\sigma / \rho_{L}\right)_{2}^{1 / 2}}{\left(\sigma / \rho_{L}\right)_{1}^{1 / 2}}\left(\frac{g_{1}}{g_{2}}\right)^{1 / 2}
$$

We $\times$ Fr leads to

$$
\frac{U^{4} \rho_{L}}{8 \sigma}=\text { equal }
$$

$$
\text { or } \quad \frac{U_{2}}{U_{1}}=\frac{\left(\sigma / \rho_{L}\right)_{2}^{1 / 2}}{\left(\sigma / \rho_{L}\right)_{1}^{1 / 2}}\left(\frac{L_{1}}{\mathrm{~L}_{2}}\right)^{1 / 2}
$$

$\operatorname{Re}^{4} \mathrm{Fr} / \mathrm{We}^{3}$ leads to

$$
\frac{\rho_{L} \sigma^{3}}{8 \mu_{L}^{4}}=Q=\text { equal }(2.36) \quad \text { or } \quad \frac{\left(\mu_{L}\right)_{2}}{\left(\mu_{L}\right)_{1}}=\frac{\left(\rho_{L} \sigma\right)_{2}^{1 / 2}}{\left(\rho_{L} \sigma\right)_{1}^{1 / 2}}\left(\frac{L_{2}}{L_{1}}\right)^{1 / 2}
$$

$Q$ is the liquid-property number, so called since for constant $g$ it depends solely on the 1 iquid properties. The remaining three requirements determine the scale factor for pressure, mean molecular welght, and gas viscosity: 


$$
\begin{array}{llll}
\frac{p_{G, \text { char }}}{\rho_{L} U^{2}}=\text { equal } & \text { (2.38) } & \text { or } & \frac{\left(p_{G, \text { char }}\right)_{2}}{\left(p_{G, \text { char }}\right)_{1}}=\frac{\left(\sigma \rho_{L}\right)_{2}}{\left(\sigma \rho_{L}\right)_{1}} \frac{L_{1}}{L_{2}} \\
\frac{\rho_{G, \text { char }}}{\rho_{L}}=\text { equal } & (2.40) & \text { or } & \frac{M_{2}}{M_{1}}=\frac{\left(T \rho_{L} / \sigma\right)_{2}}{\left(T \rho_{L} / \sigma\right)} \frac{L_{2}}{L_{1}}
\end{array}
$$

With the help of (2.38) and the perfect gas law:

$\mathrm{p}_{\mathrm{G}} / \mathrm{P}_{\mathrm{G}}=\mathrm{RT} / \mathrm{M}(\mathrm{R}=$ gas constant, $\mathrm{T}$ = temperature, $\mathrm{M}=$ mean molecular welght, here the masses in kilograms/mole is used).

$$
\frac{\mu_{G}}{\mu_{L}}=\text { equal (2.42) or } \quad \frac{\left(\mu_{G}\right)_{2}}{\left(\mu_{G}\right)_{1}}=\frac{\left(\mu_{L}\right)_{2}}{\left(\mu_{L}\right)_{1}}
$$

\subsubsection{Validity of the conventional equations}

The assumptions underlying the conventional equations (2.1) - (2.10) are the following (Chesters 1978).

- 1 - a surfactant free, Newtonian liquid. The treatment of a large class of liquids as incompressible and Newtonian is accurately justifled.

The liquids used in this investigation are very pure and refreshed regulary so that the concentration of surface active agents is very low. Because the diffusion process by which the surface active agents are transported to the gas-liquid surface is very slow, the influence will be small especially in flows where fresh surface is formed continuously (plug or slug flow). Changes in flow pattern (foaming), void fraction and pressure drop are observed (Oya 1971, Geld v. d. 1985), if surface active agents are added to the liquid.

- 2 - a perfect, Newtonian gas. If the vapour content of the gas is low this assumption is a good approximation. The vapour pressure is always relatively low because the temperature of the liquid is well below the boiling point and the system pressure is high.

- 3 - no mass transfer between the phases. Under the clrcumstance described above the mass transfer is negligible.

- 4 - isothermal flow. Strictly a contradiction exists between the assumption of isothermal flow for the gaseous phase and the existence of pressure 
varlations. However, because large pressure variations are generally the result of large velocities, which in turn results in fine dispersion of the phases, sufficient time for conduction of heat into the gaseous phase probably exist to justify this approximation in many flows.

In many flow situations also the influence of viscous dissipation can be neglected.

- 5 - no velocity discontinuity at phase boundaries. Except for highly rarefied gases the absence of slip at the boundarles is a valid assumption. - 6 - no influence of liquid-solid contact angle. In many flow configurations the solid bounding surfaces are always totally wet so that a contact angle is absent. Where it is not, the influence of the three-phase contact Ine is often a minor one except for very small tubes at low gravities (Barnea et al. 1983, Biswas and Greenfield 1985).

- 7 - no special effects during the rupture of thin film or filaments of either phase. Chesters (1975) showed that the influence of van der waals forces on the final thinning of atomising liquid sheets and of the liquid film between coalescing bubbles is negligible. Cooling of the film or filaments by increase of surface area per unit volume can increase the surface tension locally and so decreases the thinning rate.

Except for cases where atomizing liquid sheets and coalescence of gas bubbles dominate the flow the similarity criteria derived from the conventional equations are valid.

\subsection{SATISFACTION OF THE CRITERIA}

For isothermal, incompressible, single-phase flows with prescribed boundary velocities the only similarity criterion besides geometrical similarity is equality of Reynolds number, and this may be satisfied in many different ways: different fluids, different length scales and an appropriate velocity scale; the same fluid, different length and velocity scales, etc. For isothermal two-phase flows six parameters must be equalized and the situation is much less flexible. It will emerge that for $g$ constant only certain liquids can scale each other and that the choice of the scalant liquid completely determines the length and velocity scale factors. If $g$ is variable, as can be achieved with a rotating model, or if some of the parameters are unimportant, the situation becomes more flexible. 


\subsubsection{Constant gravity}

Requirement (2.36) indicates that only certain liquids can scale each other. Some values of the liquid-property number $Q$ at $20^{\circ} \mathrm{C}$ are given in Table 2.1 and it is seen that by a slight adjustment of the temperature of either water or trichloroethylene (and hence of $Q$ ) these two liquids are possible scalants. The properties of mercury-gas or alcohol-gas flow, however, cannot be simulated by any of the other liquids tabulated.

\begin{tabular}{|c|c|c|c|c|c|}
\hline LIQUID & $\begin{array}{c}\rho_{L} \\
\mathrm{~kg} / \mathrm{m}^{3} \\
10^{3}\end{array}$ & $\begin{array}{c}\sigma \\
N / m \\
10^{-3}\end{array}$ & $\begin{array}{c}\mu_{L} \\
\mathrm{~Pa} \cdot \mathrm{s} \\
10^{-3}\end{array}$ & $\begin{array}{l}Q \\
10^{8}\end{array}$ & $\begin{array}{c}\left(\sigma / \rho_{1}\right)^{1 / 2} \\
\mathrm{~m}^{3 / 2} / \mathrm{s} \\
10^{-3}\end{array}$ \\
\hline Mercury & 13.6 & 472 & 1.56 & 24600 & 5.9 \\
\hline Water & 1.00 & 72.8 & 1.00 & 390 & 8.5 \\
\hline Trichloroethylene & 1.46 & 29.3 & 0.56 & 380 & 4.5 \\
\hline Propanol I & 0.81 & 24 & 2.26 & 0.44 & 5.4 \\
\hline Water $/ 38 \%$ glyc. & 1.09 & 71.2 & 3.35 & 3.18 & 8.1 \\
\hline Gasoll & 0.84 & 26.5 & 2.74 & 0.28 & 5.6 \\
\hline Methyl Alcohol & 0.79 & 22.6 & 0.60 & 71.7 & 5.3 \\
\hline
\end{tabular}

Table 2.1: Liquid properties $\left(20^{\circ} \mathrm{C}\right)$.

Equation (2.33) and (2.35) indicate that the choice of the liquid fixes the velocity and length scale factors. For water and trichloroethylene these are 0.72 and 0.53 . For nearly all liquids at temperatures well below the critical temperature the value of $\left(\sigma / \rho_{L}\right)^{1 / 2}$ lies between $3 \times 10^{-3}$ and $9 \times 10^{-3}$. Consequently the length scale factor 13 limited to about 0.3 at constant gravity.

\section{$2.3 .2 \mathrm{~g}$ variable: rotating model}

At constant gravity the length scale factor given by (2.33) is of the order of unity for most liquid pairs and a considerable scale down of a two-phase system is consequently not possible in general. If, however, the 
value of $g$ in model can be increased, by use of a rotating model, the situation changes radically (Chesters 1977). For a value of $\mathrm{g}_{2} / \mathrm{g}_{1}$ of 100 , for example, a scale down factor $L_{2} / L_{1}$ of the order 0.1 of is achleved. Thus if we assume that the 1 iquid density and the surface tension of the model fluid and original fluid do not differ very much then (2.33) becomes:

$$
\frac{\mathrm{L}_{2}}{\mathrm{~L}_{1}}=\left(\frac{\mathrm{B}_{1}}{\mathrm{~B}_{2}}\right)^{1 / 2}
$$

The consequences for the other scale factor are given below.

Equation (2.35) requires a velocity in model which is about $\left(\mathrm{L}_{1} / \mathrm{L}_{2}\right)^{1 / 2}$ greater than in the original flow. According to (2.37) the model fluid should have a considerable lower dynamic viscosity which presents no problems unless the viscosity of the original liquid is low (smaller than $10^{-3} \mathrm{~Pa} . \mathrm{s}$ ). The requirement (2.39) leads to a greater absolute pressure in the model and (2.41) indicates that the model gas should ideally have a much lower molecular weight than the original gas. This will of ten not be possible since a molecular weight smaller than 2 is unattainable. In this event one of the two basic requirements, $\mathrm{p}_{\mathrm{G}} / \rho_{\mathrm{G}} \mathrm{U}^{2}=$ equal or $\rho_{\mathrm{G}}$, char ${ }^{\prime \rho_{\mathrm{L}}}=$ equal must be dropped. Equality of the Euler number takes account of the compressibility of the gas and is important if either appreciable expansion of the flow occurs over distances of interest or pressure waves play an important role in the flow. The present method is restricted in its applicability to approximately horizontal flows where both expansion and pressure waves will often be negligible, certainly as regarded their influence on the local flow pattern.

Equality of the density ratio takes account of the influences of gas inertial forces on the liquid flow, which largely determine the development of interfacial waves. If the modelling of compressibility is relinquished the pressure scaling requirement (2.39) disappears. The requirement of equal density ratio can now be satisfied by adapting the scale factor for absolute pressure. The equations (2.39) and (2.41) are then replaced by:

$$
\frac{\left(p_{G, \text { char }}\right)_{2}}{\left(p_{G, \text { char }}\right)_{1}}=\frac{\left(p_{L} T / M\right)_{2}}{\left(p_{L} T / M\right)_{1}}
$$


Clearly however it is sensible to choose the value of $M_{2} / M_{1}$ and hence $\left(\mathrm{p}_{\mathrm{G}, \text { char }}\right)_{2} /\left(\mathrm{p}_{\mathrm{G}, \text { char }}\right)_{1}$ as close as possible to the 1 deal values $\mathrm{g}$ iven by (2.39) and (2.41) since the greater the departure, the greater the influence of compressibility in the model and at some stage this will become appreclable. The influence of the Euler number has been tested experimentally by using different model gases.

Finally (2.43) requires a model gas with a much lower viscosity than the gas of the original system. Because of the relatively small variation of $\mu$ among gases $\left(10 \times 10^{-6}<\mu_{G}<30 \times 10^{-6} \mathrm{~Pa} . \mathrm{s}\right)$ and the limitations in choice of gases imposed by (2.45), it will generally not be possible to satisfy this requirement. The influence of gas viscosity in many two-phase flows is very slight however.

\subsubsection{Consequences of the use of rotation to increase gravity}

The motion of a fluid with respect to a steadily rotating axis can be described by the usual equations (2.1) - (2.10) provided the body force per unit mass of the fluid, $\underline{g}$, is:

$$
\underline{g}=\underline{g}_{0}-2 \underline{\omega} \times \underline{u}-\underline{\omega} \times(\underline{\omega} \times \underline{r})
$$

where $\underline{r}$ is the position vector of the element of fluid concerned (the origin lying on the axis of rotation, see figure 2.2) and $\underline{\omega}$ is the angular velocity vector of the system, $\underline{g}_{0}$ is the acceleration of natural gravity, $-2 \underline{w} \times \underline{u}$ is the Coriolis force per unit mass which is seen to be zero if the local velocity vector of the fluid $\underline{u}$ is parallel to $\underline{\omega}$ and $-\underline{\omega} \times(\underline{\omega} \times \underline{r}$ ) is the centrifugal force directed radially outwards with magnitude $R \omega^{2}$ ( $R$ is the perpendicular distance from the point concerned to the axis of rotation).

Variation of the gravity over the flow

For large values of $\omega(2.46)$ becomes approximately:

$$
\underline{\underline{g}}=-\underline{\omega} \times(\underline{\omega} \times \underline{r})=\omega^{2} \underline{R}
$$

Thus for $\underline{g}$ to be approximately constant over the flow concerned, this flow may only have appreciable extension in the direction parallel to the axis of 
rotation and not in the radial direction. This confines the method of the scaling to approximately horizontal flows:

$$
\frac{D}{R} \ll 1
$$

where $D$ is the diameter of the tube. From $D=0.005 \mathrm{~m}$ and $R=0.5 \mathrm{~m}$ the ratio $D / R$ becomes 0.01 which might be small enough to neglect the variation of the gravity in the radial direction.
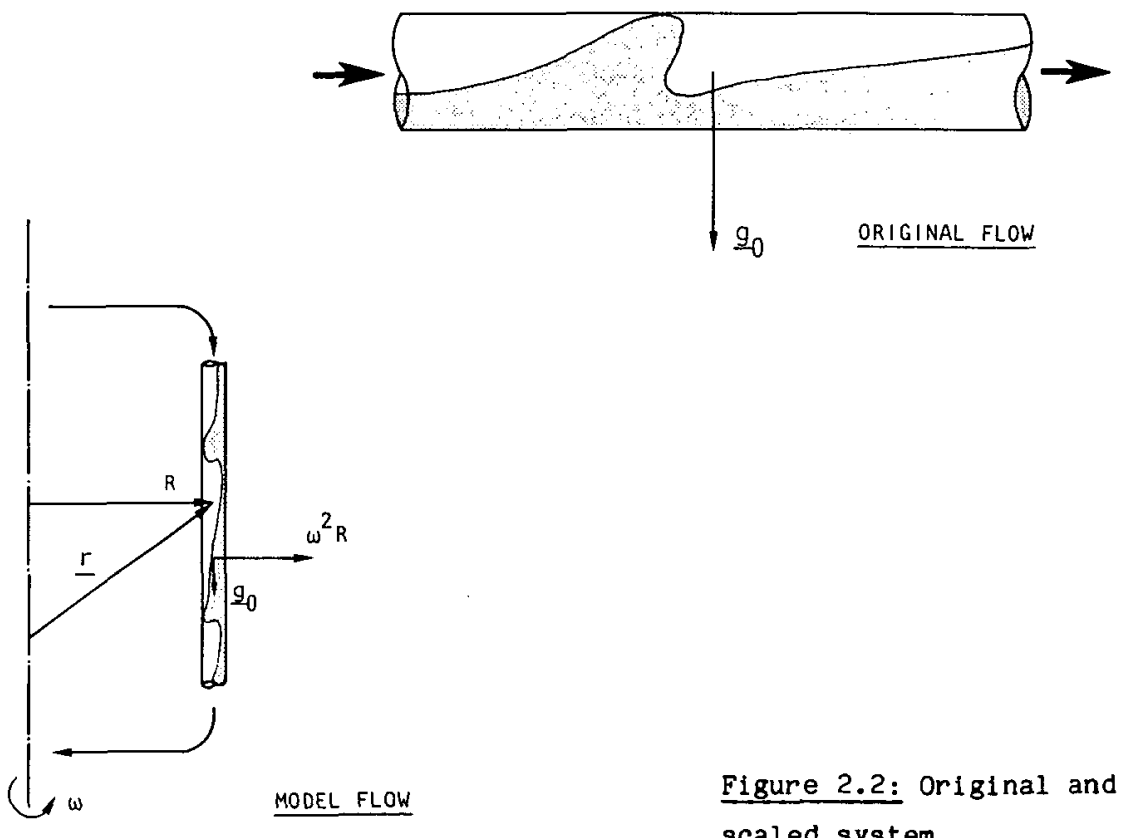

Figure 2.2: Original and scaled system.

Influence of the natural gravity

For reason of symmetry the axis of rotation is chosen to be vertical, parallel to the direction of natural gravitational acceleration.

Consequently the flow in the rotating test section is subjected to a small downward inclination (the mean direction of the flow is in the direction of $\left.\mathrm{g}_{0}\right)$. As indicated in section 1.3 the flow pattern transitions are very sensitive to the inclination of the tube. In order to fulfil the requirement of geometrical similarity the original large-scale flow is subjected to the same downward inclination $\left(\approx \mathrm{g}_{0} / \mathrm{g}\right.$ radians) as the model flow. The small

21 
inclination causes an additional (gravitational) pressure drop over the test section. To obtain the frictional pressure drop the total pressure drop must be corrected for the gravitational pressure drop. Therefore the void fraction must be determined experimentally or theoretically.

The influence of natural gravity could also be removed by inclining the model flow to the axis of rotation. This would however increase the influences of radial extension and Coriolis forces and might not be worth the gain. In addition the inclination angle must then be adjusted if the rotation rate is changed.

\section{Influence of Coriolis forces}

The Coriolis force produces a transverse component of $\underline{g}$ (either radial or circumferential or both depending on the instaneous local value of $\underline{u}$ ). For a flow strictly parallel to the axis of rotation, such as stratified or annular flow, this force is absent but in general a lateral component of velocity, $v$, will exist giving rise to a corlolis component. For the Coriolis force to be negligible:

$$
\frac{2 \omega \mathrm{v}}{\omega^{2} \mathrm{R}}=\frac{2 \mathrm{v}}{\omega \mathrm{R}} \ll 1
$$

With $\omega=50$ and $R=0.5,(2.49)$ leads to $v \ll 12 \mathrm{~m} / \mathrm{s}$.

The largest velocity fluctuations are to be expected in intermittent flows. Kvernvold (1984) showed that the translation speed of a slug is about 1.3 times the mean velocity of the liquid in the slug. The latter one is equal to the mixture velocity, which is the sum of the superficial gas and liquid velocity. In the present investigation the maximum mixture velocity with slug flow was $17 \mathrm{~m} / \mathrm{s}$. Since $v$ is a typical lateral component of velocity it may be expected to be an order of magnitude smaller than the axial velocity. Nevertheless the Coriolis force can be about $15 \%$ of the centrifugal force, and therefore the experimental conditions are suspect at intermittent flows. At low gas velocities the Coriolis forces are negligible.

\subsection{MEASUREMENT PROGRAMME}

For testing the similarity criteria (2.21) - (2.25) and (2.30) two tests, $A$ and $B$, with different length scale factors have been performed. 
The scaling test A includes measurements of pressure drop, vold fraction and flow pattern for a length scale factor of 0.1 . Much attention is paid to the geometrical similarity of the stationary $50 \mathrm{~mm}$ diameter test section and the rotating $5 \mathrm{~mm}$ diameter test section (the test sections are of ten indicated by the nominal tube diameter). Both sections are 200 diameters 1 ong and the tube walls are hydrodynamically smooth for the experimental region of interest. The same inclination angle and the same inlet geometry is applied and in both sections pressure drop is measured over 40 diameters after an entry length of 120 diameters. The capacitive sensors for measuring vold fraction are geometrically similar and the calibration curves are almost the same. The time-varying vold signal and if only pressure drop is measured, the time-varying pressure drop signal are used to establish the flow pattern. The dimensionless numbers are varied with respect to the values of the ideal scaling (see section 2.4.2).

For the scaling test B a $100 \mathrm{~mm}$ diameter stationary test section and the two test sectlons mentloned above are used, allowing a length scale factor of 0.05 and 0.5 . The dimensionless length and distance between pressure taps is quite different from the other two sections. For measuring vold fraction a different method has been used.

The test series are denoted by a capital letter with a number as subcript, indicating the overall circumstances (fluid properties, temperature, gravity, pressure etc.) under which the measurements are carried out. A test series contalns several test conditions, denoted by a small letter and two figures as subscript. The first figure indicates the adjusted liquid velocity, the second one the gas velocity, see Appendix I and II. For the scaling test $A$ eighteen test conditions are examined and 429 runs are incorporated in the results; the test $B$ includes 18 conditions and 155 runs.

\subsubsection{The scaling test $A$}

A length scale factor of 0.10 is realized via a rotating $5 \mathrm{~mm}$ diameter tube (for calculation $D=4.90 \times 10^{-3} \mathrm{~m}$ ) and a stationary $50 \mathrm{~mm}$ diameter tube (for calculations $D=49.8 \times 10^{-3} \mathrm{~m}$ ). It is desirable to use water in the rotating test section to prevent changing of the fluid properties, by leakage of cooling water into the circuit. For the large scale flow a mixture of water and glycerine at $20^{\circ} \mathrm{C}$ is used. The required viscosity of the 
mixture is calculated from (2.37). The factor $\left(\sigma \rho_{L}\right)_{1}^{1 / 2} /\left(\sigma \rho_{L}\right)_{2}^{1 / 2}$ is ranging from 1.00 to 1.06 for all possible mixtures of water and glycerine. Hence to the first approximation:

$$
\left(\mu_{L}\right)_{1}=1.03\left(\mu_{L}\right)_{2}\left(L_{1} / L_{2}\right)^{1 / 2}=3.3 \times 10^{-3} \mathrm{~Pa} . \mathrm{s}
$$

which corresponds to a solution of $38 \%$ glycerine by welght (for fluid properties see table 2.1 or Appendix I table I-4).

The effective gravitational acceleration in the model can be calculated from (2.33): $\mathrm{g}_{2} / \mathrm{g}_{1}=113$ which requires a rotation rate of 460 revolutions per minute. With the help of equation (2.35) the velocity scale factor becomes: $U_{2} / U_{1}=3.33$. The volume flow scales with $\mathrm{UL}^{2}$, hence the gas and liquid flows in the model are $U_{2} L_{2}^{2} / U_{1} L_{1}^{2}=0.032$ times the flows in the original system. The frequency scale factor is $\left(\mathrm{U}_{2} / \mathrm{L}_{2}\right) /\left(\mathrm{U}_{1} / \mathrm{L}_{1}\right)=33.8$. All processes in the model flow are a factor of 33.8 faster than those in the original flow.

The ideal ratio of the molecular welght is 0.090 according to $(2.41)$. With helium $\left(M=4 \times 10^{-3} \mathrm{~kg} / \mathrm{mole}\right)$ in the model flow and carbon dioxide $(M=$ $\left.44 \times 10^{-3} \mathrm{~kg} / \mathrm{mole}\right)$ in the original flow this value would almost be obtained. However because of the high flow rates in the $50 \mathrm{~mm}$ section it is preferable to use air ( $M=29 \times 10^{-3} \mathrm{~kg} / \mathrm{mole}$ ) instead of carbon dioxide. In this case $M_{2} / M_{1}=0.138$. The scale factor for the absolute pressure (see 2.45) becomes $\left(\mathrm{p}_{\mathrm{G}, \text { char }}\right)_{2} /\left(\mathrm{p}_{\mathrm{G}, \text { char }}\right)_{1}=6.61$. The ratio of the Euler number now is: Eu $/ E u_{1}$ $=0.66$. The absolute pressure in either the stationary test section or rotating test section can however be chosen freely. High pressure ( $2.95 \mathrm{MPa})$ is applied to the rotating test section for the following reasons:

- High centrifugal accelerations are practicable. The system pressure must be always higher than the (hydrostatic) pressure drop $\rho_{L} \omega^{2} R^{2} / 2$ in the radial part of the rotor (see figure 4.7), otherwise an undesirable under pressure in the liquid circuit arises. At 1500 revolutions per minute the pressure drop in the radial part is $2.8 \mathrm{MPa}$.

- At high pressures only a relatively small amount of gas, which is dissolved in the water during the two-phase flow, comes free in the radial part of the rotor and hence the disturbance of the flow is relatively small. The pressure decreases considerably when the liquid flows, see figure 4.7, from the separator to the axis of the rotor, especially at high rotation 
speeds. Due to the pressure decrease the dissolved gas comes free in the 11 quid outlet and the filling degree of the separator increases. Large amounts of gas in the liquid outlet can also disturb the flow and pressure balance (the density of the fluid in the outlet decreases and hence the hydrostatic pressure drop).

- The expansion of the gas is relatively small at high pressures and hence the Euler number is less important.

- The Influence of the Euler number can easily be tested by decreasing the pressure (the equality of density ratio can still be satisfled by using gases with different molecular weights).

- Pressure fluctuations due to fluctuations of the liquid level in the separator can be neglected. The pressure in the test section is the sum of the gas outlet pressure and the hydrostatic pressure drop of the liquid column above the outlet of the test section in the separator. The liquid level in the separator changes due to leakage of cooling water into the circuit or leakage of the circult itself, see section 4.1.4.

The last requirement of equal viscosity ratio (2.42) cannot be fulfilled. The dynamic viscosity of helium $\left(19.6 \times 10^{-6} \mathrm{Pa.s}\right)$ is about three times the ideal value.

To compare frictional pressure drops in model and original flow, the socalled friction multiplier $\phi_{L}^{2}$ is used, which is the ratio of the frictional pressure gradient, $(\Delta \mathrm{p} / \Delta \mathrm{L})_{\mathrm{f}}$, to the single-phase liquid pressure gradient $(\Delta \mathrm{p} / \Delta \mathrm{L})_{\mathrm{L}}\left(=\mathrm{f}(\mathrm{Re}) \rho_{\mathrm{L}} \mathrm{U}_{\mathrm{L}}^{2} / 2 \mathrm{D}\right)$.

Strictly speaking the pressure gradient should be made dimensionless with $\rho_{L} U^{2} / L$, however if the Reynolds number is equal in both flows the two approaches are equivalent $\left(U=U_{L}, L=D\right)$. The scale factor for the pressure drop $\Delta p / \Delta L$ can be calculated from $(2.38)$ :

$$
\frac{(\Delta p / \Delta L)_{2}}{(\Delta p / \Delta L)_{1}}=\frac{\left(\rho_{L} U^{2} / D\right)_{2}}{\left(\rho_{L} U^{2} / D\right)_{1}}=103
$$

The values of the fluid velocities, single-phase liquid pressure drop and the dimensionless groups for the 18 test conditions are listed in table $I-1$ (50 mm, test series $A_{1}$ ) and table $I-2\left(5 \mathrm{~mm}\right.$, test series $A_{7}$ ) of Appendlx $I$. The fluld properties can be found in table $I_{-4}$ and table $I-7$. 
Summarizing: a two-phase flow of air-water $/ 38 \%$ glycerine in a $50 \mathrm{~mm}$ diameter pipe with a downward inclination of $1: 113$ and an absolute pressure of $0.44 \mathrm{MPa}$ is scaled with a helium-water flow in a $5 \mathrm{~mm}$ diameter tube at 2.95 MPa and a gravity of 113 times natural gravity. Of the six similarity parameters the Reynolds number, the Froude number, the Weber number and the density ratio are equal in both systems.

In the next section the variation of individual dimensionless groups is considered in order to investigate thelr separate influences.

\subsubsection{Variation of the dimensionless groups}

The system pressure and rotation rate for the scaling test $A$ are chosen in such a way that the individual dimensionless groups can be changed relative easily.

Variation of the dimensionless groups for the rotating test section: - Froude number - In horizontal flow this number is an important parameter. The gravitational forces act on the liquid phase causing it to be displaced towards the bottom of the tube. Increasing the relative importance of gravity will lead to a better separation of the phases and probably lower pressure drops and lower vold fractions. By increasing or decreasing the gravity the inclination angle is changed, as a result of which flow pattern transition can be provoked (this effect is only important at low flow velocities). For the test series $A_{13}\left(A_{14}\right)$ the Froude number is $1.77(0.46)$ times the value required for scaling (test series $A_{7}$ ), see Appendix I table I-6 and table I -7 .

- Reynolds number - By decreasing the viscosity of the Ilquid the Reynolds number is raised by a factor of 2 (test series $A_{12}$ ). Of the remaining dimensionless groups only the Weber number $(+9 \%)$ and the Euler number $(+12 \%)$ are affected. The required experiments are performed at $55^{\circ} \mathrm{C}$. The vapour pressure of the water is still low compared with the system pressure at this temperature.

- Density ratio - The effect of the density of the gas is tested by using air (test series $A_{11}$ ) instead of helium. A mixture of helium with $14 \%$ air by volume (test series $A_{10}$ ) is also applied. 
- Euler number - The Euler number is varied by lowering the gas pressure. In order to retain the same density of the gas, the molecular weight is raised. Air (test series $A_{9}$ ) and a helium/air mixture are used (test series $A_{8}$ ).

Variation of the dimensionless groups for the stationary test section:

- Density ratio - By decreasing the pressure from $0.44 \mathrm{MPa}$ to atmospheric both the density ratio and the Euler number are lowered by a factor of four (see test serie $A_{3}$, In table I-3 and I-4 of Appendix I). In many two-phase flows the Euler number is not an important parameter so that the influence is principaliy that of the gas density. Except for the Weber number (- 11\%) the other dimensionless groups are preserved.

- Reynolds number - This number is raised by a factor of 3 by using water instead of a mixture of water and glycerine (see test series $A_{5}$ ). - Weber number - By using propanol at $16^{\circ} \mathrm{C}$ the Weber number is raised by a factor of 2.2 (test series $A_{6}$ ). The kinematic viscosities of propanol and the water/glycerine mixture are equal, hence the Reynolds number is just as the other dimensionless groups unchanged.

In the $50 \mathrm{~mm}$ test loop the influence of the inclination angle and filling degree of the separator on the flow have also been studied while by measuring pressure drop over different sections, it was checked whether the flow had reached a fully developed form: see section 5.2 .

\subsubsection{The scaling test $B$}

Starting from the test conditions and fluid properties of the $100 \mathrm{~mm}$ test section the conditions and fluid properties for the rotating $5 \mathrm{~mm}$ section (length scale factor of 0.05 ) and for the stationary $50 \mathrm{~mm}$ section (1ength scale factor of 0.5 ) are calculated from the similarity criteria. A review of the test conditions and fluid properties of the test series $B_{1}, B_{2}, B_{3}$ and $B_{4}$ is given in Appendix II.

The measurements with the $100 \mathrm{~mm}$ test $100 \mathrm{p}$ were carried out at the Koninklijke/Shell Laboratorium in Amsterdam (KSLA). Pressure drop, void fraction and flow pattern were measured for a two-phase flow of air and gasoll, at $37^{\circ} \mathrm{C}$ and nearly atmospheric pressure. 


\section{Length scale factor of 0.05}

Taking the exact diameter of $D_{1}=105.5 \times 10^{-3} \mathrm{~m}$ and $D_{2}=4.9 \times 10^{-3} \mathrm{~m}$ the precise value of $L_{2} / L_{1}$ is 0.0464 . If we require water to be used as liquid scalant then the equations (2.33), (2.35) and (2.37) lead to the following scale factors:

$$
8_{2} / g_{1}=1071, U_{2} / U_{1}=7 \text { and }\left(\mu_{L}\right)_{2} /\left(\mu_{L}\right)_{1}=0.39
$$

With water at a temperature of $18^{\circ} \mathrm{C}$ it would be possible to fulfil these requirements. However the required rotation rate is high and a gas pressure of at least $2.50 \mathrm{MPa}$ is required to avold under pressure and hence flashing of the liquid in the circuit. This large pressure cannot be applied without violating the requirement of equal density ratio (2.40). If helium is used in the rotating test section (hydrogen is not considered for safety reasons) the scale factor for the system pressure becomes 8.1 with the help of (2.45) and $M_{1} / M_{2}=0.138$. The system pressure in the original flow is $0.11 \mathrm{MPa}$, hence for the model flow an absolute pressure of $0.89 \mathrm{MPa} 1 \mathrm{~s}$ required. The maximum allowable centrifugal acceleration $\omega^{2} \mathrm{R}$, corresponding to $a$. hydrostatic pressure drop $\Delta \mathrm{P}$ in the radial part of the rotor can be calculated from:

$$
\Delta P=\rho_{L} \omega^{2} R^{2} / 2
$$

where $R$ is the radial distance between the tube axis and the axis of the rotor $(R=0.475 \mathrm{~m})$. Using $\Delta P=0.89 \mathrm{MPa}$ the maximum effective gravity becomes $381 g_{0}$ ( $g_{0}$ is acceleration due to natural gravity). Ultimately the centrifugal acceleration is chosen to be $245 \mathrm{~g}_{0}$ and the hydrostatic pressure loss in the radial part of the rotor is $0.57 \mathrm{MPa}$ then. The overpressure at the connections of the pressure transducer, mounted on the axis of the rotor is $0.3 \mathrm{MPa}$ now and the volume of enclosed gas pockets is reduced, see figure 4.12 .

Because the value of $8_{2} / 8_{1}$ is not equal to the $i$ deal value given by (2.33), one of the three basic requirements of equality of the Reynolds number, Froude number or Weber number must be dropped. The liquid viscosity or the dimensionless representation of $1 t$, the Reynolds number, is responsible for the frictional shear forces while gravity causes separation of the phases, especially in horizontal flow. 
At the relatively low velocities used here, there is preponderance of the intermittent flow pattern and the Weber number will probably be less important than the Reynolds or Froude number. If atomizing liquid sheets or bubble formation are important aspects of the flow then the choice is more difficult.

The scale factor for the velocity is now derived from equality of the Froude number $\left(U^{2} / L g\right)$ :

$$
\frac{\mathrm{U}_{2}}{\mathrm{U}_{1}}=\left(\frac{\mathrm{L}_{2}}{\mathrm{~L}_{1}}\right)^{1 / 2}\left(\frac{\mathrm{g}_{2}}{\mathrm{~g}_{1}}\right)^{1 / 2}
$$

and the scale factor for the (kinematic) viscosity is obtained from equality of the Reynolds number:

$$
\frac{\left(\mu_{L} / \rho_{L}\right)_{2}}{\left(\mu_{L} / \rho_{L}\right)_{1}}=\frac{U_{2} L_{2}}{U_{1} L_{1}}
$$

The equations (2.52) and (2.53) yleld $U_{2} / U_{1}=3.37$ and the value of $\left(\mu_{L} / \rho_{L}\right)_{2}$ becomes $0.509 \times 10^{-6}$, which can be satisfied with water at a temperature of $55^{\circ} \mathrm{C}\left(\rho_{\mathrm{L}}=986 \mathrm{~kg} / \mathrm{m}^{3}, \mu_{\mathrm{L}}=0.50 \times 10^{-3} \mathrm{~Pa} . \mathrm{s}, \sigma=67.1 \times 10^{-3 .} \mathrm{N} / \mathrm{m}\right)$.

The ratio of the weber number in the model and original flow is 0.23 . The required pressure scale factor is 9.00 (from 2.45) and hence the absolute pressure in model is $0.99 \mathrm{MPa}$.

Summarizing: a two-phase flow of air and gasoll in a $100 \mathrm{~mm}$ diameter pipe at $37^{\circ} \mathrm{C}$ and $0.11 \mathrm{MPa}$ is scaled by a helium-water flow in a $5 \mathrm{~mm}$ diameter pipe at $55^{\circ} \mathrm{C}$ and $0.99 \mathrm{MPa}$ and a centrifugal acceleration of $245 \mathrm{~g}_{0}$. The Froude number, Reynolds number and density ratio are equal in both flows.

\section{Length scale factor of 0.5}

Comparing the $50 \mathrm{~mm}$ and the $100 \mathrm{~mm}$ stationary sections a length scale factor of 0.472 is obtained. From (2.33) and $g_{2}=g_{1}$ it follows that: $\left(\sigma / \rho_{L}\right)_{2}^{1 / 2}=0.472 \times\left(\sigma / \rho_{L}\right)_{1}^{1 / 2}=2.65 \times 10^{-3^{2}}$. Most 1 lquids have a larger value of $\left(\sigma / \rho_{L}\right)^{T / 2}$ and again the requirement of equal weber number is relinquished. The velocity scale factor now becomes 0.68 (from 2.52) and the scale factor for the kinematic viscosity is 0.32 (from 2.53). Water at $18^{\circ} \mathrm{C}$ can then be used as liquid scalant. 
If air is used in the $50 \mathrm{~mm}$ section, the requirement of equal density ratio can be satisfied by $P_{2} / p_{1}=1.12$ (from 2.45 and $M_{2} / M_{1}=1$ ). If a small deviation (18\%) of the density ratio from the 1 deal value is accepted then the pressure in the model can be atmospheric (series $B_{3}$ ), otherwise the absolute pressure is $0.123 \mathrm{MPa}$ (series $\mathrm{B}_{2}$ ).

\subsection{CONCLUSIONS}

For dynamic similarity of two-phase flows in addition to geometrical similarity of imposed boundary conditions six dimensionless groups must be equal. These are the Reynolds, Froude, Weber and Euler number together with the ratio of gas to liquid densities and dynamic viscosities.

If the effective acceleration due to gravity $g$ is constant only small length scale factors are possible in general. The choice of the liquids then determine the length, velocity and pressure scale factor as well as the required molecular weight and viscosity of the gas scalant.

By using a rotating model the effective value of $g$ can artificially be increased and large scale down factors become feasible. For practical reasons the method is however restricted to horizontal or nearly horizontal flow.

Two scaling tests are carried out. The scaling test $A$ allows a length scale factor of 0.1 , using a stationary $50 \mathrm{~mm}$ and a rotating $5 \mathrm{~mm}$ tube. The required centrifugal acceleration is relatively $10 \mathrm{w} 113 \mathrm{~g}_{0}$, for which Coriolis forces may be of influence in some flow situations. The viscosity ratio and Euler number, which are expected to be of minor influence, are not equal in the two systems. The experimental conditions are chosen in such a way that the influence of the dimensionless groups Reynolds, Froude, Euler and density ratio can be examined relatively easily.

The starting point for the scaling test $B$ is a $100 \mathrm{~mm}$ diameter tube with a two-phase flow of air and gasoll. With the $5 \mathrm{~mm}$ and $50 \mathrm{~mm}$ test sections a length scale factor of 0.05 and 0.5 can be realized. To achleve equal Reynolds and Froude numbers, the requirement of equal weber number had to be relinquished. Moreover the Euler number and viscosity ratio are not equal in both flows. 


\section{THE SENSOR FOR VOID FRACTION MEASUREMENT}

\section{AND FLOW PATTERN IDENTIFICATION}

A new instrument has been developed for measuring void fractions in two-phase flow. To allow accurate comparisons the same method is used in the 5 and $50 \mathrm{~mm}$ test section. The principle of the device is based on the measurement of the electrical capacitance of the two-phase mixture, see section 3.1. The electrode configuration consists of a helical cross capacitor in which is placed an acrylate tube (see section 3.2). In section 3.3 the capacitance and electric field of a capacitor with electrodes flush with the wall are calculated. The influence of the acrylate tube on the calibration curves for annular flow and dispersed flow is then determined theoretically for a cylindrical cross-capacitor (section 3.4). Experimental callbration curves for annular flow and stratifled flow are given in section 3.4.1 and 3.4.3. Section 3.4.5 deals with the measurement strategy for intermittent flows. Finally section 3.5 outlines how the sensor is used for flow pattern identification.

\subsection{BACKGROUND OF THE IMPEDANCE METHOD}

An important parameter for making comparisons in two-phase flows is the vold fraction $\alpha$, defined here as the fraction of a certain channel volume that is occupied by the gas phase. The time-averaged as well as the instantaneous values are used. The device which has to be used here must fulfil the following requirements:

- the method must be simple and the meter must withstand severe environmental conditions since high centrifugal forces and high pressures are to be dealt with.

- the flow may not be disturbed by the meter.

- the meter must be suitable for flow pattern identification.

From the three main techniques for measuring mean phase content: radioactive absorption and scattering, direct volume measurement by quick-closing valves and impedance measurements only the latter is relatively easy to use in a rotating system. The second requirement can be satisfied by using electrodes which are embedded in the wall. The last demand limits the axial 
extension of the electrodes and requires a quick response of the measuring system.

The determination of vold fraction from electric impedance measurements has been widely applied for gas-water mixtures. Many of the basic techniques are reviewed by Hewitt (1978). The major difficulty with the impedance gauges is the sensitivity to flow pattern. Section 3.3 pays attention to this problem. If impedance gauges are operating in conditions in which conductance is dominating, drift occurs due to changes in liquid conductivity. For water an increase in temperature from $25^{\circ} \mathrm{C}$ to $50^{\circ} \mathrm{C}$ doubles the conductivity whereas the relative permittivity decreases only $15 \%$ (Hill 1969). Moreover the change of ionic concentration has no influence on the relative permittivity of the water. Drift can be avoided by operating at sufficiently high frequency to give domination by capacitarice. A standard $1 \mathrm{MHz}$ capacitance meter is used. This frequency is well below the value where dispersion effects become important.

Capacitance sensors with different electrode configurations have been examined by Gregory (1973), Abouelwafa (1980) and Borst (1983). The helical configuration seems to give the best results. Two thin brass strips are wound helically around a thin acrylate pipe section in such a way that they are always opposite each other. Abouelwafa and Kendall (1979) showed that the relationship between the capacitance and the void fraction is linear and independent of the flow pattern and relative permittivity of the media. For the acrylate inner tube a correction factor is introduced which decreases with decreasing thickness of the tube. In glaring contrast with the work of Abouelwafa and Kendall it is shown in this study that the thickness and relative permittivity of the acrylate tube are very important design quantities especially for media with a high relative permittivity such as water.

The two additional shield electrodes fixe stray-capacitance and make an analytical approach of the helical cross capacitor possible. At both sides of the helical electrodes, guard electrodes are placed to render edge effects negligible. Often a rotating electric field is used to obtain better spatial averaging (Merilo, 1977 and v.d. Geld, 1985). With a helical electrode configuration, however, spatial averaging is automatically realized and only a simple capacitance meter is needed. 


\subsection{CONSTRUCTION OF THE SENSOR}

The sensors for the 5 and $50 \mathrm{~mm}$ test section are geometrically similar, except for some small differences due to limitations in their manufacture (for example the ratio of the width of the grooves between the electrodes and the width of the electrodes is much larger for the $5 \mathrm{~mm}$ sensor than for the $50 \mathrm{~mm}$ sensor). The similarity of the sensors has the advantage that the callbration curves are very like and accurate comparisons of the two flows are possible.

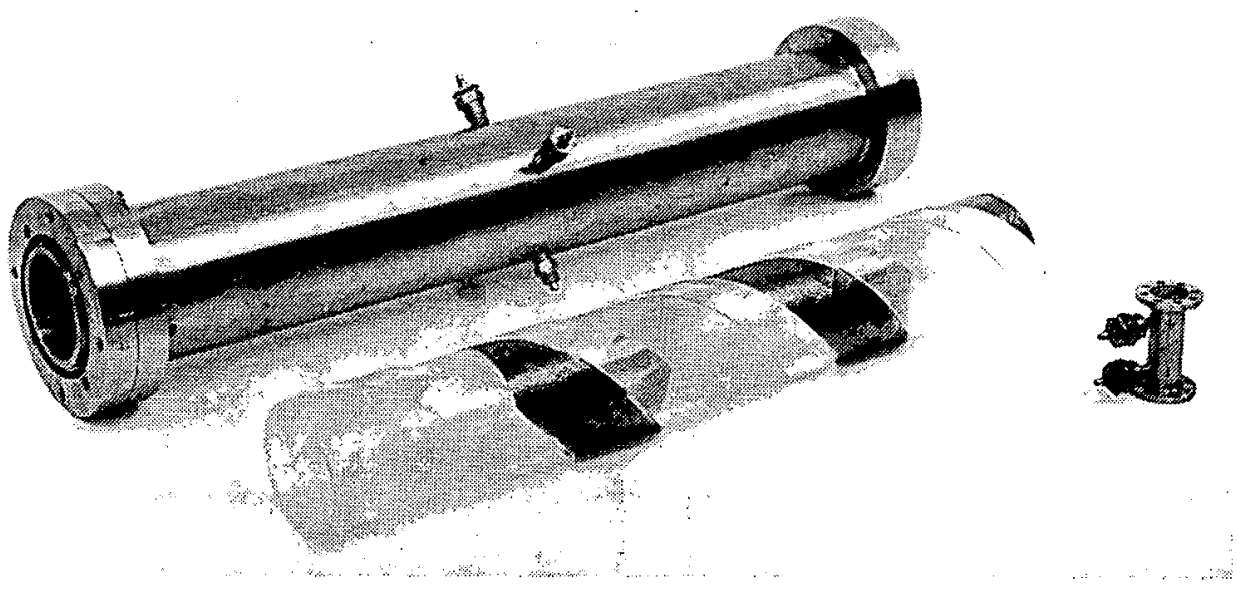

Figure 3.1: The 50 and $5 \mathrm{~mm}$ void sensors.

A photo of the $50 \mathrm{~mm}$ sensor with the acrylate inner tube $1 \mathrm{~s}$ given in figure 3.1. The starting point for manufacturing is an acrylate tube with an inner diameter of $50.0 \mathrm{~mm}$ and an outer diameter of $60 \mathrm{~mm}$. At the positions of the two electrodes 1 and 2 the outer diameter of the tube is turned until the desired thickness of the wall is obtained. Then tape with a copper coating is winded around the tube. Electrode 1 makes three complete revolutions around the tube, electrode 2 only two (see figure 3.1). The pitch of the helix is $\pi D$ hence the active length of the sensor is $2 \pi D$ $(\approx 6$ diameters). Between the electrodes, two shield electrodes are wounded around the tube. At both ends of electrode 2 a guard-electrode is placed to 
render edge effects negligible. The guard electrodes are connected electrically to the two shield electrodes. The acrylate tube is placed inside a stainless steel pipe (the shield tube) with the appropriate flanges.

Minfature coaxial cable connectors are screwed into the thread ports of the shield tube in such a way that the spring loaded center wire of the coaxial connector makes contact with the electrode. The shield of the coaxial cable is connected to the shield, the shield electrodes and the guard electrodes. Using this arrangement the measured capacitance is independent of the length of the coaxial leads and external fields. To prevent rupture of the sensor tube the space between the sensor tube and the shield tube is maintained at the same pressure as the pressure in the separator.

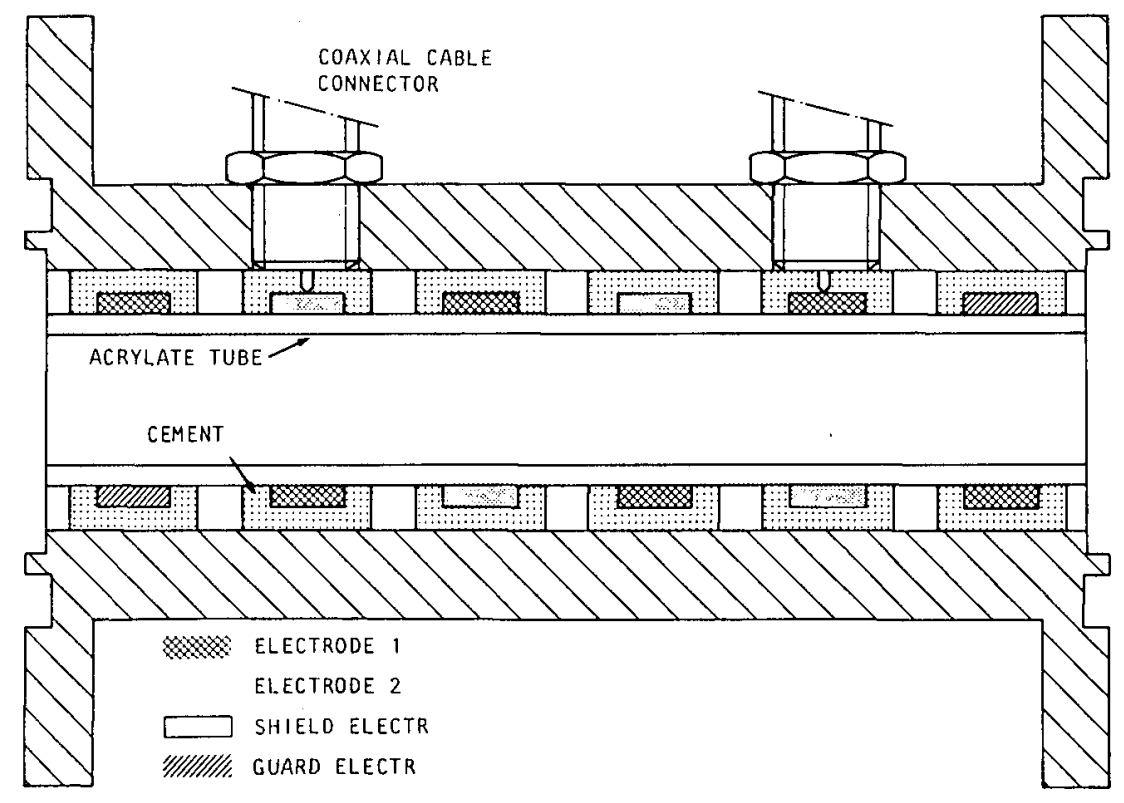

Eigure 3.2: The void meter for the rotating test section.

The manufacturing of the $5 \mathrm{~mm}$ sensor and the $50 \mathrm{~mm}$ sensor with electrodes flush with the wall is totally different from the procedure described above. Now the starting point is a stainless steel rod in which deep helical grooves are machined, see figure 3.3 . 


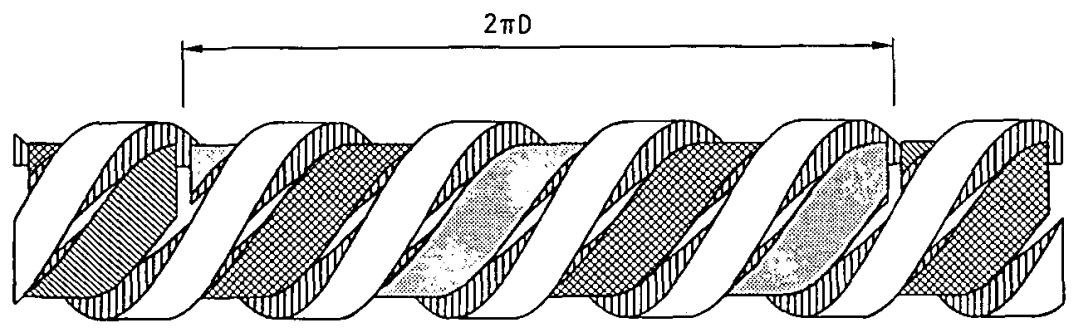

Figure 3.3: Stainless steel rod with helical grooves.

The rod with grooves is cemented in a stainless steel tube with the approplate flanges and thread ports for the electrical connections. The two shield electrodes and guard electrodes are connected to the shield tube by small stainless steel pins. Next the hole for the two-phase flow is drilled centrally into the rod in such a way that the electrodes 1 and 2 are severed from the shield electrodes. The electrodes are then totally embedded in the insulating cement, see figure 3.2. Finally the acrylate inner tube with the appropriate inner and outer diameter is cemented into the tube. The sensors machined in this way can withstand high pressures and high centrifugal accelerations. A detailed description of the sensors is given by Borst (1985).

The electrodes 1 and 2 are connected to the terminals of a capacitance meter (Boonton model $72 \mathrm{BD}$ ). The shield electrodes and guard electrodes are connected to the earth of the meter. The capacitance meter makes rapid three-terminal measurements with a phase-sensitive detector at a frequency of $1 \mathrm{MHz}$. The three-terminal principle allows accurate measurements in the presence of relative large values of shunt capacitance to earth. The phase-sensitive detector effectively ignores capacitor losses due to conduction as long as the quality, $\omega C / K$, is larger than 1 . Here $\omega$ is the angular frequency of the electric fleld, $C$ the capacitance being measured and $K$ the conductance of the sensor.

For the $50 \mathrm{~mm}$ sensor with electrodes flush with the wall, filled with distilled water (specific conductivity $x=2.5 \times 10^{-4} 1 / \Omega \mathrm{m}$ ) the capacitance is $120 \mathrm{pF}$, the conductance is $40 \times 10^{-6} 1 / \Omega$ and hence the quality is 19. If the specific conductivity is ralsed to $50 \times 10^{-4} 1 / \Omega \mathrm{m}$, the capacitance decreases 
by only 1\%. The behavior of the sensor with an acrylate inner tube is totally different because of the so-called Maxwell-wagner effect, see Appendix III. If the specific conductivity of the water is raised from $2.5 \times 10^{-4}$ to $50 \times 10^{-4} 1 / \Omega \mathrm{m}$ the capacitance of the liquid filled sensor increases by about 10\%. The calibration curves remain the same however and without knowing the precise value of the conductivity accurate void measurements are still possible. The specific conductivity of the liquid in the rotating test section is always less than $40 \times 10^{-4} 1 / \Omega \mathrm{m}$ and in the stationary test section less than $5 \times 10^{-4} 1 / 8 \mathrm{~m}$.

\subsection{THE CAPACITANCE OF THE HELICAL CROSS-CAPACITOR}

Our objective is to calculate the electric field and capacitance of a hellcal cross-capacitor with electrodes flush with the wall. The cross-section for $z=0$ is depleted in figure 3.4 .

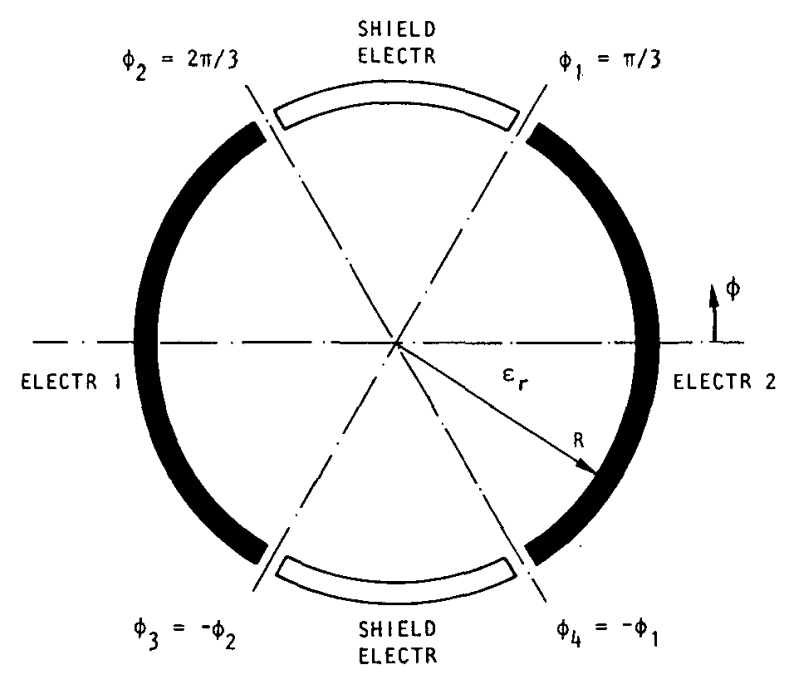

Figure 3.4: Cross-section of the helical cross-capacitor.

To electrode 1 a potential of $V_{0}$ Volt is applied, the electrode 2 and the shield electrodes are connected to earth. At any cross-sectional plane the 
elctrode configuration is identical except for an angular rotation $z / p R$, where $z$ is the co-ordinate in the axial direction. The pitch parameter $p$ is equal to the ratio of the pitch $s$ of the helix and the circumference $2 \pi R$ : $p=s / 2 \pi R$.

The potential distribution at the electrodes is given by:

$$
\begin{aligned}
& \mathrm{V}=\mathrm{V}_{0} \text { for } \quad|\phi-\mathrm{z} / \mathrm{pR}|<\phi_{1} \\
& V=0 \text { for } \quad \phi_{1}<|\phi-z / p R|<\pi
\end{aligned}
$$

The Fourier expansion of the above distribution 1s:

$$
V(R, \phi, z)=\frac{V_{0} \phi_{1}}{\pi}+\frac{2 V_{0}}{\pi} \sum_{n=1}^{\infty} \frac{\sin \left(n \phi_{1}\right) \cos (n(\phi-z / p R))}{n}
$$

To obtain the capacitance, the Laplace equation in cylindrical co-ordinates has to be solved:

$$
\frac{\partial^{2} V}{\partial r^{2}}+\frac{1}{r} \frac{\partial V}{\partial r}+\frac{1}{r^{2}} \frac{\partial^{2} v}{\partial \phi^{2}}+\frac{\partial^{2} v}{\partial z^{2}}=0
$$

For reasons of symmetry the distribution of the potential in any cross-sectional plane is the same except for an angular rotation $z / p R$. Hence to solve the Laplace equation the variables are separated in the following way:

$$
V(r, \phi, z)=R(r) \times L(\phi, z)
$$

Equation (3.3) then yields:

$$
\frac{1}{R}\left(\frac{d^{2} R}{d r^{2}}+\frac{1}{r} \frac{d R}{d r}\right)+\frac{1}{r^{2} L} \frac{\partial^{2} L}{\partial \phi^{2}}+\frac{1}{L} \frac{\partial^{2} L}{\partial z^{2}}=0
$$

The general solution of the electrostatic potential distribution is:

$$
\begin{aligned}
& V=\left(A_{0}+B_{0} \phi+B_{0}^{\prime} z\right)\left(C_{0}+D_{0} \ln z\right)+ \\
& \sum_{n=1}^{\infty}\left(A_{n} \cos (\sigma \phi-q z)+B_{n} \sin (\sigma \phi-q z)\right)\left(C_{n} I_{\sigma}(q r)+D_{n} K_{\sigma}(q r)\right)
\end{aligned}
$$


where $I_{\sigma}(q r)$ and $K_{\sigma}(q r)$ are known as the modified Bessel-functions of the first and second kind and of order $\sigma$. The necessity for $V$ to be finite. results in $D_{n}=0$ and the periodicity of the solution with respect to $\phi$ and 2 leads to $\sigma=m, q=n / p R, B_{0}=0$ and $B_{0}^{\prime}=0$. Symmetry of the solution with respect to $\xi=0$, where $\xi=\phi-z / p R$ results in $B_{n}=0$. The potential now becomes:

$$
V(r, \xi)=F_{0}+\sum_{n=1}^{\infty} F_{n} \cos (n \xi) I_{n}\left(\frac{n r}{p R}\right)
$$

where $F_{0}=A_{0} C_{0}$ and $F_{n}=A_{n} C_{n}$.

Satisfying the boundary condition (3.2) the final solution is obtained (Borst, 1985):

$$
V(r, \xi)=\frac{\xi_{1} V_{0}}{\pi}+\frac{2 V_{0}}{\pi} \sum_{n=1}^{\infty} \frac{\sin \left(n \xi_{1}\right) \cos (n \xi) I_{n}\left(\frac{n r}{p R}\right)}{n I_{n}\left(\frac{n}{p}\right)}
$$

The electric field inside the helical cross-capacitor is calculated from:

$$
E_{r}=-\frac{\partial V}{\partial r}, E_{\phi}=-\frac{1}{\dot{r}} \frac{\partial V}{\partial \xi} \frac{\partial \xi}{\partial \phi} \text { and } E_{z}=-\frac{\partial V}{\partial \xi} \frac{\partial \xi}{\partial z}
$$

Figure 3.5 and figure 3.6 show the electric fleld in a cross-sectional plane and in an axial plane for a sensor filled with a homogeneous dielectric material. The length of the arrow is proportional to the magnitude of the component of the electric field in the cross-sectional plane. Large gradients of the electric field are present only in the nelghbourhood of the shield electrodes. However these field lines do not contribute to the capacitance. The internal cross-capacitance per unit length $C^{\prime}$ is:

$$
C^{\prime}=\frac{l}{L}\left|\frac{Q}{V_{0}}\right|=\frac{1}{V_{0}}{\stackrel{\phi}{\phi_{2}}}_{\varepsilon_{0}} \varepsilon_{0} \varepsilon_{r} E_{r=R} \operatorname{Rd} \phi
$$

Using (3.8) and (3.9) the final expression for $C^{\prime}$ is:

$$
C^{\prime}=\frac{2 \varepsilon_{0} \varepsilon_{r}}{\pi}\left(\ln \frac{\sin \left(\left(\phi_{1}+\phi_{2}\right) / 2\right)}{\sin \left(\left(\phi_{2}-\phi_{1}\right) / 2\right)}+\sum_{n=1}^{\infty} \frac{2 \sin \left(n \phi_{1}\right) \sin \left(n \phi_{2}\right) I_{n+1}\left(\frac{n}{p}\right)}{p n I_{n}\left(\frac{n}{p}\right)}\right)
$$




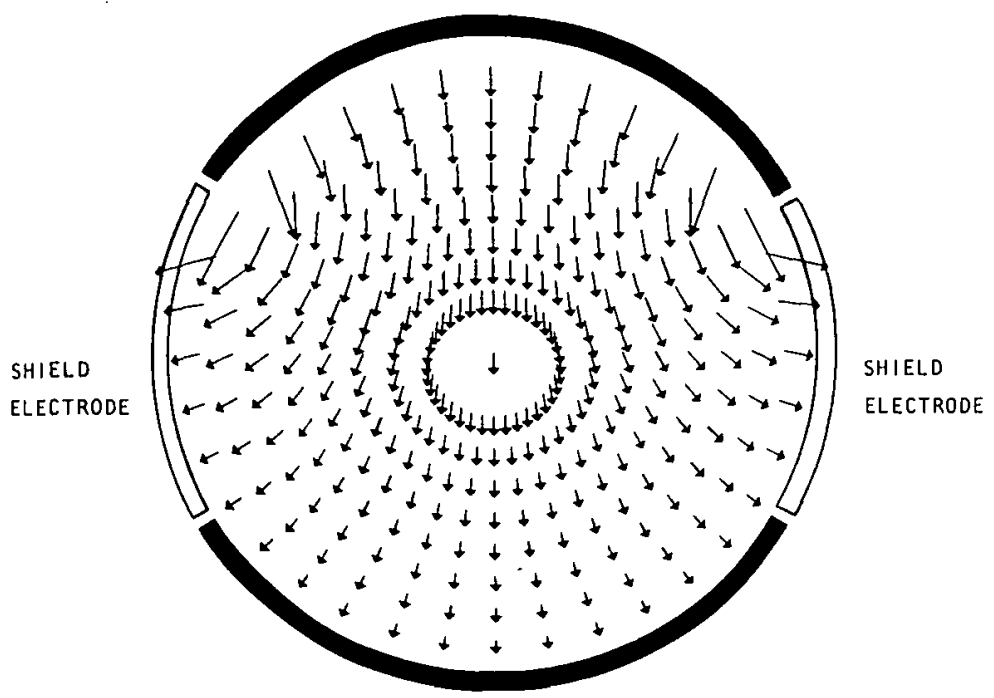

ELECTRODE 2

Figure 3.5: Electric field in a cross-sectional plane.

Figure 3.6: Electric field in an axial plane. 
In $\mathrm{f}$ igure 3.7 the capacitance per unit length as a function of the angle $\phi_{1}\left(2 \phi_{1} R\right.$ is the width of the electrodes) for three values of the pitch parameter $p$ is given. At large values of $p$ the capacitance of the cylindrical cross-capacitor is obtained. At values of the pitch parameter larger than 1 the influence of the helix form is small.

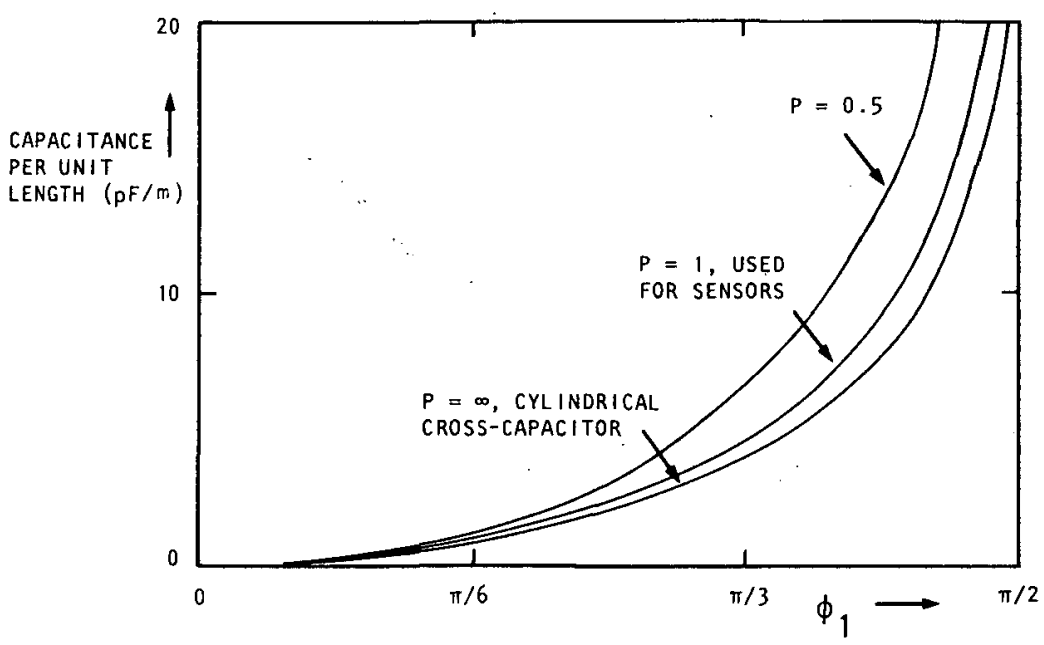

Figure 3.7: Capacitance per unit length of the helical cross-capacitor.

The 5 and $50 \mathrm{~mm}$ void sensors have the following design values $\phi_{1}=\pi / 3$, $\phi_{2}=2 \pi / 3, p=1$ and the calculated capacitance per unit length is 4.54 $\mathrm{pF} / \mathrm{m}$. The measured capacitance of the $50 \mathrm{~mm}$ helix cross capacitor deviates only $3 \%$ from the predicted value $(1.43 \mathrm{pF}$ at an active electrode length of $2 \pi D)$.

The measured capacitance of the $5 \mathrm{~mm}$ sensor is $25 \%$ smaller than the predicted value $(0.14 \mathrm{pF})$ because the electrodes are actually much smaller than the design values. Moreover the width of the grooves between the electrodes is about one fifth of the electrode width, and this is not negligibly small.

If the sensor is filled with an inhomogeneous dielectric material or a mixture of two dielectric materials the field lines will be distorted and in 
general the capacitance cannot be determined analytically. However in speclal cases, such as idealized annular flow or Idealized dispersed bubble flow an analytical approach is possible. In section 3.4 .1 and 3.4 .2 the capacitance of a cylindrical cross-capacitor ( = helical cross-capacitor with $p=\infty$ ) is calculated for these two cases.

\subsection{CALIBRATION CURVES}

For cylindrically symmetrical flow the sensor may be modelled by a cylindrical cross-capacitor filled with symmetrically placed dielectric cylinders. The results and analysis for a helical cross-capacitor are almost the same but the algebraic efforts are much larger. In section 3.4 .1 the capacitance of an idealized annular flow is calculated and compared with the experimental curves. The influence of the acrylate sensor tube is examined. The theoretical calibration curves for dispersed bubble flow are presented in 3.4 .2 and the experimental curves for stratified flow in 3.4.3. Finally in section 3.4 .4 the measurement strategy for intermittent flow is outlined.

\subsubsection{Annular flow}

Consider a cylindrical cross-capacitor of circular cross-section as shown in figure 3.8. The configuration simulates an ldealized annular flow (cylindrical symmetry and the absence of waves on the surface). The space inside the cyindrical electrodes is divided into three regions:

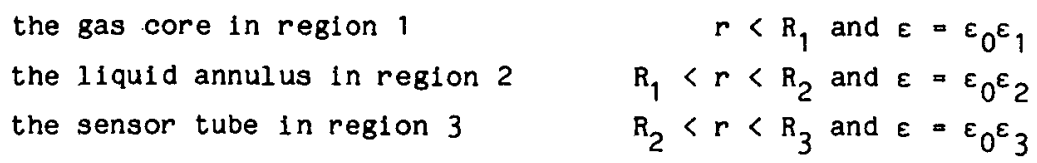

Our objective is to calculate the internal cross-capacitance per unit length, $C^{\prime}$, between electrode 1 and 2. To electrode 1 a potential of $v_{0}$ volt is applied, while electrode 2 and the shield electrodes are earthed. The Laplace equation in two dimensions in cylindrical co-ordinates is:

$$
\frac{\partial^{2} V}{\partial r^{2}}+\frac{1}{r} \frac{\partial V}{\partial r}+\frac{1}{r^{2}} \frac{\partial^{2} V}{\partial \phi^{2}}=0
$$




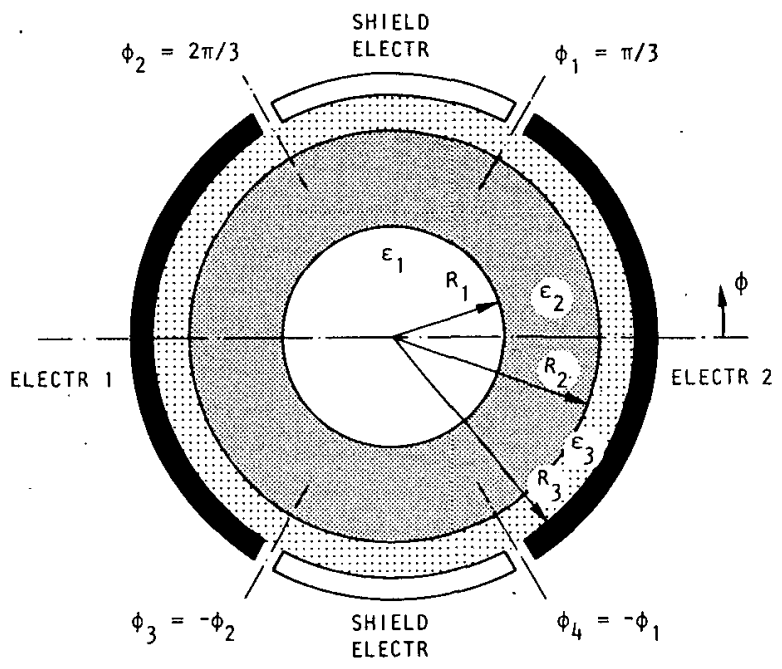

Figure 3.8: Cross-section of the cylindrical cross-capacitor.

The general form of the electrostatic potential distribution within the capacitor, obtained as a solution of (3.11) is:

$$
\begin{aligned}
V(r, \phi)= & \left(A_{0}+B_{0} \phi\right)\left(C_{0} \ln r+D_{0}\right)+ \\
& \sum_{n=1}^{\infty}\left(\left(C_{n} r^{n}+D_{n} r^{-n}\right)\left(A_{n} \cos (n \phi)+B_{n} \sin (n \phi)\right)\right)
\end{aligned}
$$

Taking into account of symmetry with respect to $\phi$ and the necessity for $V$ to be $f$ inite, the expressions for $V(r, \phi)$ in the three reglons are:

$$
\begin{aligned}
& \text { Region } 1 \quad V_{1}(r, \phi)=A_{10}+\sum_{n=1}^{\infty} A_{1 n} r^{n} \cos (n \phi) \\
& \text { Region } 2 \quad V_{2}(r, \phi)=A_{20}+B_{20} \ln r+\sum_{n=1}^{\infty}\left(A_{2 n} r^{n}+B_{2 n} r^{-n}\right) \cos (n \phi) \\
& \text { Region } 3 \quad V_{3}(r, \phi)=A_{30}+B_{30} \ln r+\sum_{n=1}^{\infty}\left(A_{3 n} r^{n}+B_{3 n} r^{-n}\right) \cos (n \phi)
\end{aligned}
$$


The boundary conditions for the problem are:

- Equal potential at the boundary between

region 1 and 2: $\quad V_{1}\left(R_{1}, \phi\right)=V_{2}\left(R_{1}, \phi\right)$

region 2 and 3 : $\quad V_{2}\left(R_{2}, \phi\right)=V_{3}\left(R_{2}, \phi\right)$

- Potential distribution at the electrode surface obtained from (3.2) with $p=\infty$ :

$v_{3}\left(R_{3}, \phi\right)=\frac{v_{0} \phi_{1}}{\pi}+\frac{2 v_{0}}{\pi} \sum_{n=1}^{\infty} \frac{\sin \left(n \phi_{1}\right) \cos (n \phi)}{n}$

- Continuity of the radial component of the displacement at the boundary between

region 1 and 2: $\quad \varepsilon_{0} \varepsilon_{1}\left(\frac{\partial V_{1}}{\partial r}\right)_{r=R_{1}}=\varepsilon_{0} \varepsilon_{2}\left(\frac{\partial V_{2}}{\partial r}\right)_{r=R_{1}}$

region 2 and 3: $\quad \varepsilon_{0} \varepsilon_{2}\left(\frac{\partial V_{2}}{\partial r}\right)_{r=R_{2}}=\varepsilon_{0} \varepsilon_{3}\left(\frac{\partial V_{3}}{\partial r}\right)_{r=R_{2}}$

The solution of equation (3.16) with the help of (3.9) and (3.10) leads to the following expression for the capacitance per unit length:

$$
C^{\prime}=\frac{2 \varepsilon_{0} \varepsilon_{n}}{\pi}\left[\ln \frac{\sin \left(\left(\phi_{1}+\phi_{2}\right) / 2\right)}{\sin \left(\left(\phi_{2}-\phi_{1}\right) / 2\right)}-\sum_{n=1}^{\infty} \frac{4\left(A C F_{1}^{n}+B D\right) \sin \left(n \phi_{1}\right) \sin \left(n \phi_{2}\right)}{n\left(C D F_{2}^{n}+A B F_{3}^{n}+A C F_{1}^{n}+B D\right)}\right]
$$

where $A=\varepsilon_{1}+\varepsilon_{2}, B=\varepsilon_{2}+\varepsilon_{3}, C=\varepsilon_{3}-\varepsilon_{2}, D=\varepsilon_{2}-\varepsilon_{1}$, and $F_{1}=\left(R_{2} / R_{1}\right)^{2}$, $F_{2}=\left(R_{3} / R_{2}\right)^{2}, F_{3}=\left(R_{3} / R_{1}\right)^{2}$.

For a special case $R_{2} / R_{1}=1, \varepsilon_{1}=\varepsilon_{3}$ and $\varepsilon_{2}<5$ a solution for the capacitance is also given by Rehman e.a. (1982).

For a prescribed value of $\phi_{1}, \phi_{2}, \varepsilon_{1}, \varepsilon_{2}$ and $\varepsilon_{3}$ equation (3.22) is only a function of $R_{2} / R_{3}$ and the void fraction $\alpha=\left(R_{1} / R_{2}\right)^{2}: C=f\left(a, R_{2} / R_{3}\right)$. Since we are only interested in the relation between the relative capacitance difference, $\Delta C / \Delta C_{0}$, and the void fraction, the following expression has been calculated numerically: 


$$
\operatorname{RCD}=\frac{\Delta C}{\Delta C_{0}}=\frac{C_{m}-C_{1}}{C_{2}-C_{1}}=f\left(\alpha, \frac{R_{2}}{R_{3}}\right)
$$

where RCD is the Relative Capacitance Difference, $C_{1}, C_{2}$ and $C_{m}$ are the capacitances of the cylindrical cross-capacitor fllled with medium 1 (empty capacitor, $\alpha=1$ ) or medium 2 (liquid filled capacitor, $\alpha=0$ ) or a mixture of medium 1 and 2 .

In figure 3.9 the results of the calculations for a water annulus $\left(\varepsilon_{2}=\right.$ 80) and an air core $\left(\varepsilon_{1}=1\right)$ are shown. The curves are very sensitive to the thickness of the acrylate sensor tube $\left(\varepsilon_{3}=3.1\right)$.

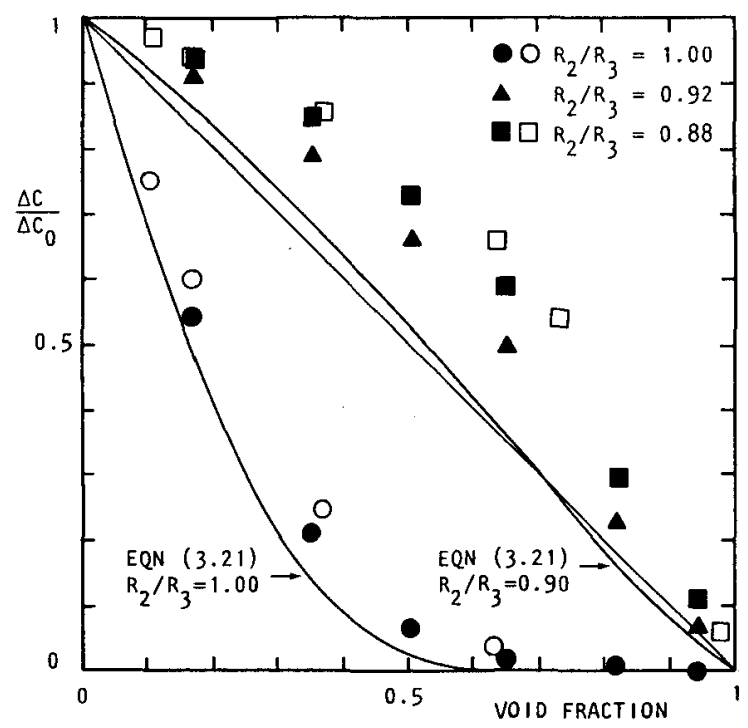

Figure 3.9: Calibration curves for annular flow, open symbols $=50 \mathrm{~mm}$, closed symbols $=5 \mathrm{~mm}$.

For $R_{2} / R_{3}=0.9$ the calculated curve is almost linear (with a glass sensor tube $\left(\varepsilon_{r}=7\right)$ the most linear curve is obtained for $R_{2} / R_{3}=0.8$ ). The relative capacitance difference for the sensor with electrodes flush with the wall $\left(R_{2} / R_{3}=1\right)$ is independent of the vold praction in the relevant vold range $\alpha>0.8$ and therefore useless. The electric field lines 
prefer going through the thin water annulus to the shield electrode rather than crossing the annulus via the gas core to the electrode 2. This effect is suppressed by placing an inner cylinder with a low relative permittivity concentrically in the sensor. The calculated curves for an acrylate core differ less than 0.02 (absolute units) from those for an air core.

The experimental calibration curves for annular flow qualitatively show the same influence of the thickness of the wall of the sensor tube, see figure 3.9. The gas core is simulated by symmetrically placed acrylate rods and the void fraction is calculated from the diameter of the rod. In the vold range $0.2<\alpha<0.8$ the experimental points are 0.1 to 0.2 higher than the calculated ones. This may be caused by the use of acrylate mock ups instead of air. However the calculated curves for an acrylate core, differ less than 0.02 from those for an air core.

The relative capacitance difference with the rods placed against the wall of the sensor tube, is about 0.05 smaller. This implies that crossing of the field ines from electrode 1 to electrode 2 is hindered then.

In this investigation most flow patterns are stratified or can be regarded as being locally stratified, such as intermittent flow, see figure 3.16. Therefore the thickness of the acrylate tube is chosen in such a way that the curve for this flow type is as linear as possible. For the tests with the rotating section the sensor with $R_{2} / R_{3}=0.92$ and for the stationary test section the sensor with $R_{2} / R_{3}=0.88$ is used. In the relevant vold-range for annular flow, $\alpha>0.8$, the calibration points are reasonably close to the diagonal. The diagonal represents filling of the sensor in the vertical position. The measurements of vertical filling of the sensor deviate less than 0.02 from the dlagonal, including of the edge regions.

\subsubsection{Dispersed flow}

In the past many solutions for the relative permittivity (and conductivity) of a bulk phase containing spheres with a different permittivity have been presented. De Vries (1952) and De La Rue and Tobias (1959) compare and discuss the classical models in detail. The various solutions are all derived by solving the Laplace equation.

Maxwell (1881) considered spheres of uniform size, located in the bulk phase. If the permittivity of the spheres is small compared to the permittivity of the bulk he found: 


$$
\frac{\varepsilon_{\operatorname{mix}}}{\varepsilon_{\text {bulk }}}=\frac{1-a}{1+0.5 \alpha}
$$

where $\varepsilon_{\text {mix }}$ denotes the relative permittivity of the mixture, $\varepsilon_{\text {bulk }}$ that of the pure bulk phase and $\alpha$ the overall volume fraction. The spheres are assumed to be non-interacting and hence (3.24) is valid only for low void fractions $(\alpha<0.1)$.

Bruggeman (1935) assumed, that in concentrated mixtures the field at a given sphere is represented more accurately by using the relative permittivity of the mixture rather than that of the pure bulk phase. For low permittivity of the spheres compared to the mixture permittivity he found:

$$
\frac{\varepsilon_{\text {mix }}}{\varepsilon_{\text {bulk }}}=(1-\alpha)^{3 / 2}
$$

Although, according to the theory, equations (3.24) and (3.25) are subjected to limitations, numerous workers. Fricke and Morse (1925), De La Rue and Tobias (1959) have found that experimental data may be represented satisfactorily by the use of these relations even in moderately concentrated dispersions. Recently Sangoni and Acrivos (1983) applied a rigorous mathematical method to obtain an expression for $\varepsilon_{\mathrm{mix}} / \varepsilon_{\text {bulk }}$ which is accurate to order $\alpha^{25 / 3}$. Their solution for equal diameter spheres arranged in spatially cubic arrays indicates that (3.24) can be used for volume fractions up to 0.4 within a relative error of 2 percent.

Here equation (3.24) is used for calculating the mixture permittivity at a given vold fraction. From equation (3.22) with $\varepsilon_{2}=\varepsilon_{3}=\varepsilon_{\text {mix }}$ the relative capacitance difference is calculated. The theoretical calibration curves for air-water dispersed flow are given in figure 3.10. The thickness of the wall of the acrylate inner tube can be chosen in such a way that the calibration curve is almost linear and close to the diagonal. The experimental region of this investigation does not include dispersed flow, hence for determining the optimal thickness of the acrylate sensor tube the calibration curve for dispersed flow is not considered. 


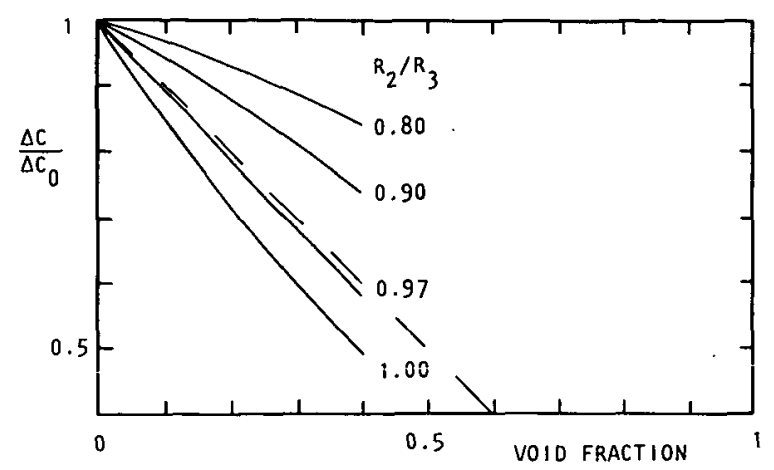

Flgure 3.10: Callbration curves for dispersed flow.

\subsubsection{Stratified flow}

For stratified flow pattern an analytical solution of the Laplace equation is not avallable. Here only experimental calibration curves are presented. To obtain the calibration curve for the $50 \mathrm{~mm}$ sensor, the tube is placed horizontal and filled with distilled water in 10 equal steps.

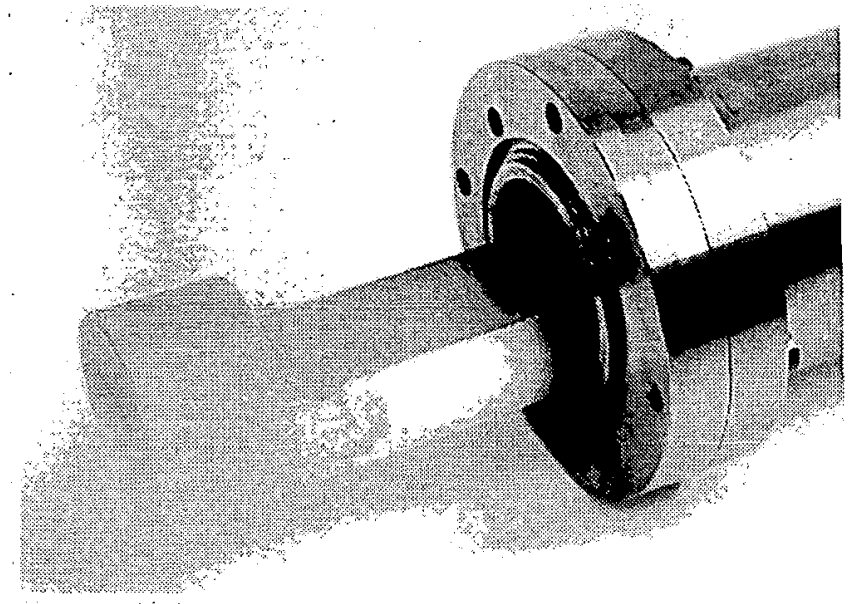

Figure 3.11: The use of acrylate mock-ups, simulating the gas.

For the $5 \mathrm{~mm}$ sensor this method is not very accurate because the liquid is not spread out very well in the small tube. Therefore mock-ups machined from acrylate rods are used to simulate the gas. The dimensions of the mock-up are measured very precisely and from that the vold fraction is 
calculated (error \pm 0.02 ). To examine the influence of the use of acrylate mock-ups this method is also applied to the $50 \mathrm{~mm}$ sensor, see figure 3.11 and compared with horizontal pilling.

Figure 3.12 and 3.13 show the experimental results for the 50 and $5 \mathrm{~mm}$ sensor. With electrodes flush with the wall the Influence of the acrylate sensor tube is qualitatively the same for both sensors. The sensitivity of the device for vold fractions in the range $\alpha>0.8$ is low. By rotating the sensor or the acrylate mock-up, the RCD fluctuates less than \pm 0.01 (50 mm) and $\pm 0.02(5 \mathrm{~mm})$. This indicates the excellent cylindrical symmetry of the sensors.

If the conductivity of the water is raised from $2.5 \times 10^{-4}$ to $50 \times 10^{-4} 1 / \Omega \mathrm{m}$ the capacitance increases about $10 \%$ due to the Maxwell wagner effect, see Appendix III. The RCD however differs less than 0.02 and hence the calibration is almost the same. A mixture of water with $38 \%$ glycerine $\left(\varepsilon_{r}=71\right)$ also does not influence $(<0.01)$ the course of the calibration curve.

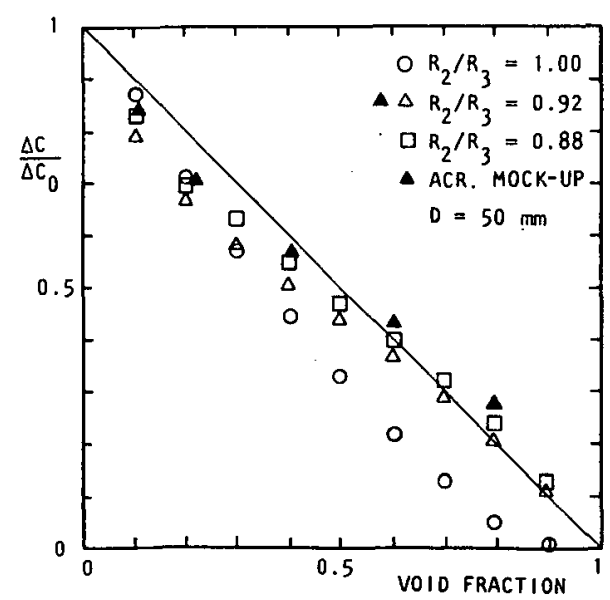

F1gure 3.12: Calibration curves for stratified flow, $50 \mathrm{~mm}$ sensor.

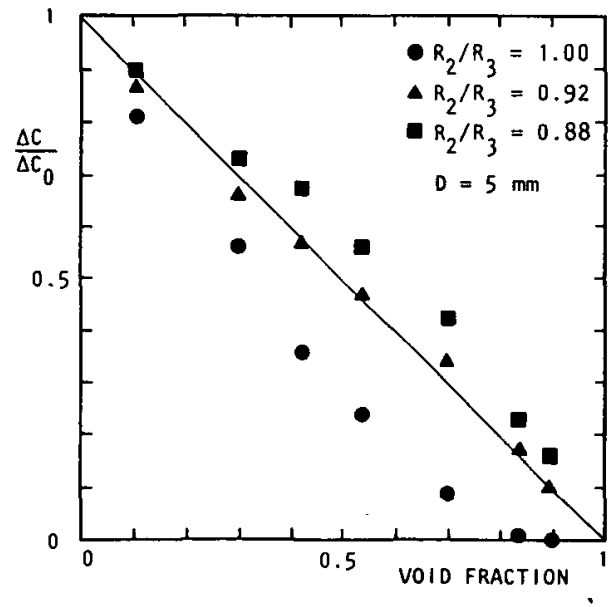

Figure 3.13: Calibration curves for stratifled flow, $5 \mathrm{~mm}$ sensor.

As can be seen from figure 3.12 the RCD of the $50 \mathrm{~mm}$ sensor with acrylate mock-ups is on the average 0.06 above the calibration curve for horizontal alr-water filling. The difference may be due to the following two effects. 
First the permittivity of acrylate is larger than that of air. The field lines are therefore more concentrated in a certaln region using acrylate instead of air. Hence with acrylate mock-ups crossing of the field lines from electrode 1 to electrode 2 is faciliated and consequently the value of the relative capacitance difference is higher. However in the case of annular flow the calculations indicate that the influence of the permittivity of the medium which is placed in the center is smali.

The second influence is the presence of a small layer of water between the mock-up and the sensor tube. The calibration curves for annular flow show that (for $R_{2} / R_{3}=0.88$ or 0.92 ) a certain volume of liquid at the wall of the tube gives a higher contribution to the RCD than the same volume in the center of the tube. Also from experiments it is found that the RCD for an acrylate rod placed at the center is about 0.05 higher than at the wall.

\subsubsection{Measurement strategy for intermittent flow}

The curves for annular flow, stratifled flow and dispersed flow of the sensors used for the scaling tests are given in figure 3.14 and 3.15 .

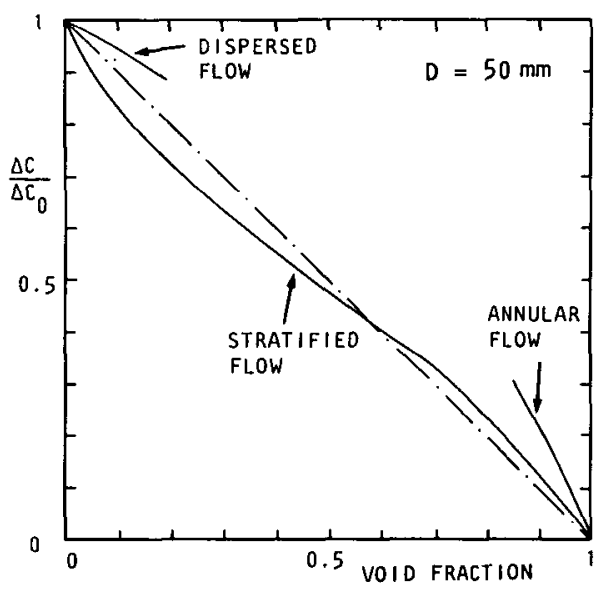

F1gure 3.14: Calibration curves for the $50 \mathrm{~mm}$ sensor, $R_{2} / R_{3}=0.88$.

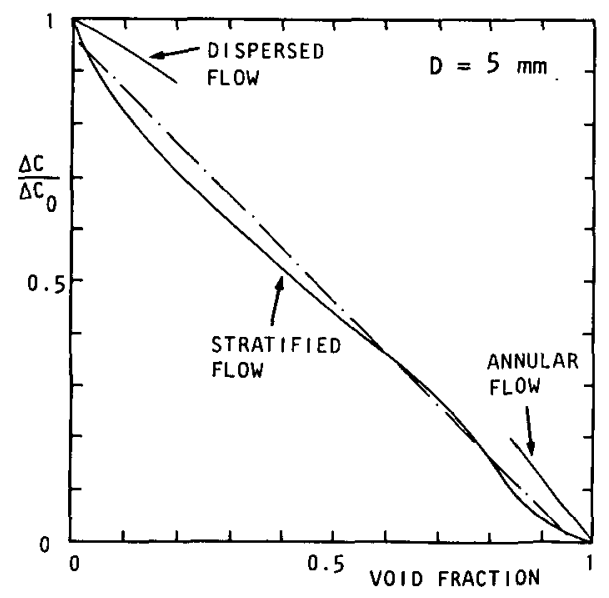

Figure 3.15: Calibration curves for the $5 \mathrm{~mm}$ sensor, $R_{2} / R_{3}=0.92$. 
The stratifled flow curve of the $5 \mathrm{~mm}$ sensor (figure 3.15) is corrected for the use of acrylate mock-ups with calibration: the values are 0.06 lower than those ( $\Lambda$ ) of figure 3.13, except for $\alpha=0$ and $\alpha=1$.

Ideally the sensor would have the same calibration curve for all flow patterns, and if this curve is linear a simple averaging by intergration can be applied. However this is not the case and therefore the following strategy is used.

Most flow patterns are stratified or intermittent. Locally an intermittent flow type can be regarded as being stratifled, see figure 3.16. Hence the calibration curve for stratified flow can also be used for intermittent flow.
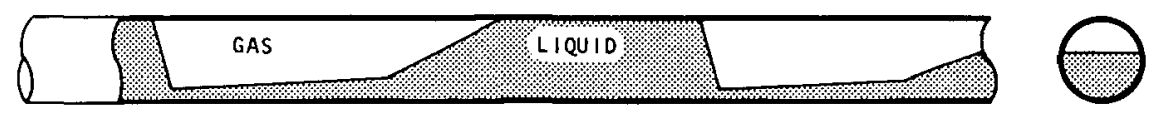

Figure 3.16: Idealized intermittent flow pattern.

If bubbles are present in a particular part of the flow, for instance behind a liquid slug, the capacitance of that part decreases according to the curve for dispersed flow. The plpe wall may be wetted after the passing of a liquid slug. In that case the liquid on the wall causes the capacitance to change according to the annular flow curve.

From visual observations it appeared that the number of bubbles in the flow is small and the liquid film at the wall is thin. The error due to the difference of the dispersed and annular flow curve from the stratifled flow curve is therefore small too.

To obtain the mean vold fraction the output signal of the capacitance meter is averaged by integration. For the $50 \mathrm{~mm}$ sensor $\alpha$ is then calculated from:

$$
\alpha_{50}=1-\operatorname{RCD}=\frac{C_{\text {mix }}-C_{11 q}}{C_{\text {gas }}-C_{11 q}}
$$

Equation (3.26) represents the diagonal in figure 3.15. If the flow is stratified the calibration curve is used. 
For the $5 \mathrm{~mm}$ sensor a line beneath the diagonal is chosen, because the callbration curve for stratified flow is below the diagonal over the whole void range.

$$
\alpha_{5}=0.96-R C D=\frac{C_{\text {mix }}-C_{119}}{C_{\text {gas }}-C_{11 q}}-0.04
$$

If the flow is stratified the calibration curve is used.

The largest deviation of the calibration curve for stratified flow from the broken line of figure 3.14 or 3.15 is 0.07 . Many intermittent flows contain stratified regions with $\alpha>0.5$ as well as stratified regions with $a<0.5$ and errors due to the non-linear callibration curve then cancel out partially. With the help of the measured time-varying vold signals (see Pigures 6.3 and 6.13 ) the likely accuracy of the mean void fraction for intermittent flows, calculated from equation 3.26 or 3.27 , is estimated. For example from the condition $a_{31}$ (figure 6.3) it can be seen that the sensor is half of the time completely filled with liquid ( $\alpha=0$ and the deviation from the broken line in figure 3.15 is +0.04 ) and half of the time only partially filled $(\alpha=0.4$ and the deviation from the broken line is negligible). The resulting error is estimated now on $0.04 / 2+0 / 2=0.02$. The maximum absolute error, determined in this way, appeared to be always less than 0.03 for the $50 \mathrm{~mm}$ as well as for the $5 \mathrm{~mm}$ sensor

\subsection{FLOW PATTERN IDENTIF ICATION FROM VOID FRACTION FLUCTUATIONS}

The sensors described in this chapter are also used for flow pattern identification. The instantaneous output signal of the capacitance meter is proportional to the vold fraction in the sensor volume. The output signal of the capacitance meter is recorded with a digital oscllloscope (Nicolet 3091). The sample frequency is $0.2 \mathrm{kHz}$ for the $50 \mathrm{~mm}$ sensor and $10 \mathrm{kHz}$ for the $5 \mathrm{~mm}$ sensor. Because the calibration curves for the two sensors are almost equal, the time varying output signal will be the same if the flow patterns are the same in both test sections. Figure 3.17 shows some recorded signals of the $50 \mathrm{~mm}$ sensor. The liquid velocity is $0.4 \mathrm{~m} / \mathrm{s}$ and the gas velocity increases from 0.3 to $10.5 \mathrm{~m} / \mathrm{s}$. 
For stratified flow the aistribution of the phases in a crossisectional plane does not change with time and hence the void fraction is constant. In this thesis the flow is called plug (PL) if the sensor is completely fllled during the passage of a "liquid wave". In this case the recorded vold fluctuations look like a block signal. If the sensor is not completely filled during the passage of the "liquid wave" then the flow is called slug (SL) and the output signals resembles on a sawtooth. If the output signal is alternating a block signal and sawtooth then the flow is indicated with PS (Plug or Slug). The semi-annular flow pattern is characterised by a thick liquid layer on the bottom and a thin film at the top. The fluctuations in the void signal are caused by waves on the liquid surface and the passage of small frothy slugs.
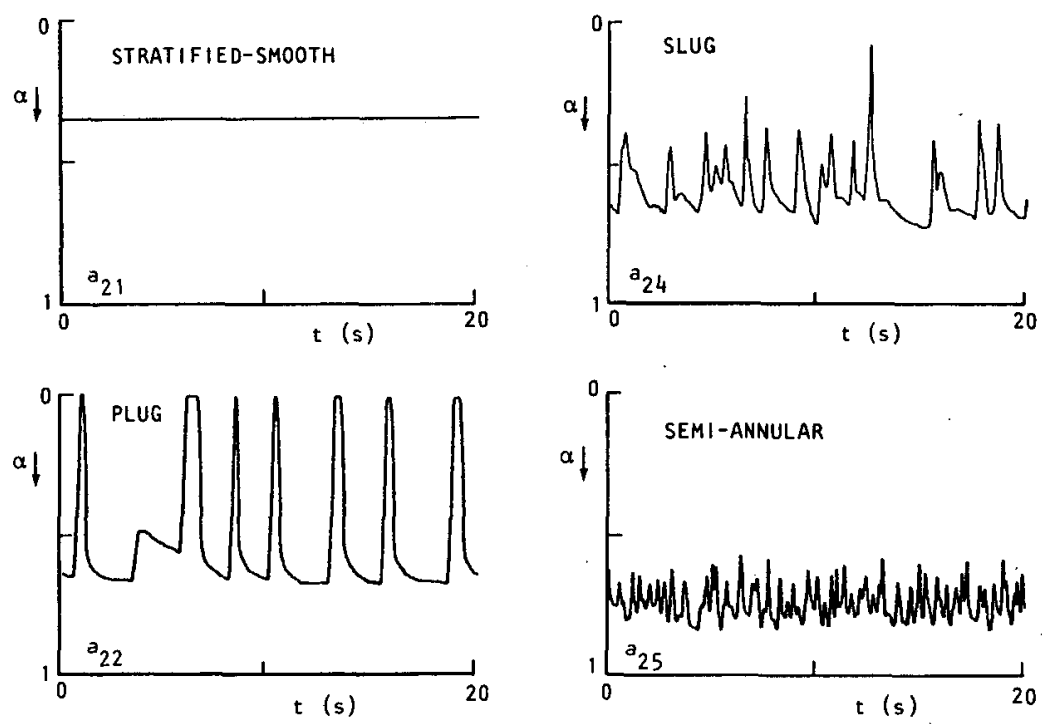

Figure 3.17: Void signal of the $50 \mathrm{~mm}$ sensor, (test series $A_{1}$, conditions $a_{21}, a_{22}, a_{24}$ and $a_{25}$ ).

At high gas velocities the vold fraction changes very rapidly. A typical time difference for the increase of the vold fraction during the entering of a slug in the $50 \mathrm{~mm}$ sensor is 0.1 seconds. For the rotating test section all processes are a factor of 34 faster than those in the stationary section. 
Hence a typical time difference for the increase of the vold praction in the $5 \mathrm{~mm}$ section is $3 \mathrm{msec}$. The response time of the capacitance meter is $2 \mathrm{~ms}$ and hence the rapid void fluctuations are not registrated well. For high flow velocities the output signal is flattened.

The slug and plug frequencies are determined simply by counting the peaks in the void signal. Two slugs are dedected apart if the distance between them is larger than the axial extension of the sensor: $U_{s} / f>6 D$, where $U_{S}$ is the translation speed of the slug, $f$ the slug or plug frequency and $6 \mathrm{D}$ the axial extension of the sensor ( $D$ is the inner diameter). The translation speed of a slug is about 1.3 times the sum of the superficial gas and liquid velocity (Gregory \& Scott 1969, Kvernvold 1984). Hence the maximum frequency that just can be measured is given by: $f_{\max }=1.3\left(U_{L}+U_{G}\right) / 6 D$. For all conditions the measured frequency is at least a factor of 3 smaller than $f_{\max }$. It is not unthinkable that sometimes two plugs or slugs are so close together that they are not detected separately. However this is more an exception than a rule and determination of the slug or plug frequency by counting the peaks is justified.

\subsection{CONCLUSIONS}

With the new vold meter, mean phase content can be determined without disturbing the hydrodynamics of the two-phase flow. Because of the helical electrode configuration spatial averaging is automatically realized and only a standard capacitance meter is used. The sensor is easy to machine for tube diameters larger than $15 \mathrm{~mm}$.

The capacitance and electric field of the helical cross-capacitor with electrodes flush with the wall is calculated for single-phase flow. For two-phase annular and dispersed flow a theoretical method is put forward for determining calibration curves for a wide range of permittivities. It is shown that the experimental calibration curve for stratified flow can also be used for intermittent flow, the maximum absolute error being \pm 0.03 .

The time-varying void signal is used for identification of the flow pattern. Because the same type of sensor is used for the 5 and $50 \mathrm{~mm}$ test section and the calibration curves are very alike, comparisons of the void fraction and flow pattern are even more accurate than one would expect from the accuracy of the devices. 


\subsection{THE CENTRIFUGE FACILITY}

The centrifuge facility which is depicted in figure 4.1 has been developed for two-phase measurements in effective gravitational fields larger than the natural gravity $\mathrm{g}_{0}$. The main components are:

- the rotor with the test section (placed parallel to the vertical shaft)

- the protection cylinder with bearings in the cover and bottom

- the v-belt drive and flexible couplings

- the mechanical seals for leakage-free passage of the fluld from the stationary circuit containing the pumps and flow meters to the rotating circuit containing the test section.

- the slip rings for output of electrical signals.

In the following paragraphs the various parts of the apparatus are discussed.

\subsubsection{The rotor}

A photo of the rotor with the lowermost cylinder dismantled is given in figure 4.2. Seven aluminium spokewheels are attached to a hollow steel shaft in which the supply lines for liquid and gas are placed. The outer diameter of the shaft is $140 \mathrm{~mm}$. Around the spokewheels two aluminium cylinders $1 \mathrm{~m}$ diameter and $1 \mathrm{~m} \mathrm{hlgh}$, are centred. Actually the cylinders are only necessary at high speeds (> $500 \mathrm{r} . \mathrm{p} . \mathrm{m}$. ) to reduce the drag. The cylinders are kept in position during rotation by pressurized flexible hoses which are sunk in the spokewheels. The flexible hoses permit a rapid installation of the cylinders. A pressure switch mounted in the central shaft automatically stops the rotation if the pressure in the hoses falls off.

The test section is attached to aluminfum plates, $3 \mathrm{~mm}$ thick which are mounted between two spokewheels. The plates run through the central shaft in which slots are machined to that end. To fix the plates and maintain the strength of the shaft, the plates are bolted to the shaft. Opposite to the test section a second section, called the dummy section, is placed in which rods can be attached to balance the weight of the fluid in the test section 


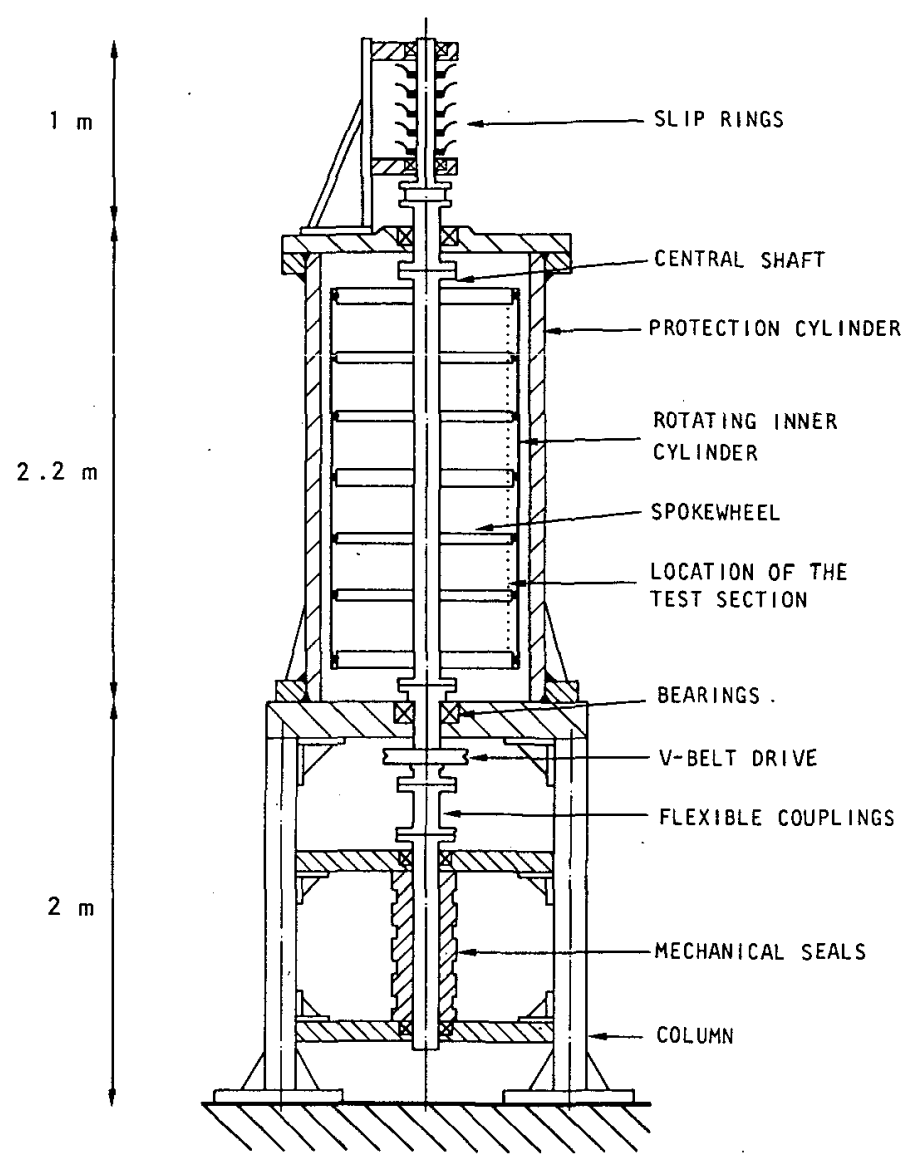

Figure 4.1: The centrifuge facility.

The separator which is located above the lowermost spokewheel is attached to the shaft with eight spokes. At $1400 \mathrm{r} . \mathrm{p} . \mathrm{m}$. the stress in the aluminium plates, spokewheels and inner cylinder is about one tenth of the yield strength. Higher rotation rates are possible if extra attention is paid to the fastening of the test section and supply lines. The power required to overcome air-friction is calculated using a correlation for the drag coefficient of rotating cylinders (Dorfman 1963, Taylor 1923). At $1400 \mathrm{r} . \mathrm{p} . \mathrm{m}$. about 3 kWatt is required. Experiments of Thomassen and v.d. Lans (1982) with a 1:20 scale model of the rotor lead to the same prediction for the drag. 


\section{Principal dimensions of the rotor}

The dimensions of the rotor depend upon the requirements concerning the two-phase flow and gravity fleld on the one hand and on the other hand upon considerations of costs and manufacturing efforts. With respect to the flow and gravity one wishes a developed two-phase flow at gravities up to $1200 \mathrm{~B}_{0}$, in which the influence of Corlolis forces and variation of the gravity over the flow should be negligible.

In many cases a two-phase flow reaches an equilibrium situation after 60 diameters of straight pipe (Hoogendoorn 1959, Welsman 1979). This does not imply however that the flow is independent of the inlet geometry. A significant effect of the inlet geometry on pressure drop and void fraction $1 \mathrm{~s}$ observed by the author in the $100 \mathrm{~mm}$ test section even at $150 \mathrm{~L} / \mathrm{D}$ from the inlet. Simpson et. al. (1977) also noted that with an L/D of 128 some differences could be observed between experiments with the mixing section at the beginning of the straight run and experiments with the mixer located before a bend which preceded the straight run.

Here the length to diameter ratio is chosen to be 200 which is sufficient to obtain fully developed flow in most cases. The same inlet geometry is aplied to the $5 \mathrm{~mm}$ and $50 \mathrm{~mm}$ test section so that eventual influences of the inlet are equal in both systems.

Because of the dependence of the centrifugal acceleration on the distance to the axis of rotation, the effective gravitational field varies over the diameter of the tube. The larger the radius of the rotor the smaller the relative change of the gravity. Further, the corlolis forces are smaller because at a given value of $g$ the angular velocity and hence the coriolis force is inversely proportional to the rotor radius.

For cost and manufacture reasons the diameter of the rotor is $11 \mathrm{mited}$ to $1 \mathrm{~m}$. The length is $2 \mathrm{~m}$, so that a $10 \mathrm{~mm}$ diameter test section with sufficlent length to diameter ratio can be used. In this investigation a $5 \mathrm{~mm}$ diameter tube is applied and oniy half of the total length is therefore in use. The value of $g$ varies by $1 \%$ over the diameter of the tube. As shown in section 2.3.3 the Corlolis forces can become important at high gas velocities. In principle, still smaller tube diameters are possible. However blockage of pressure taps and the manufacturing of the vold sensor can be a problem. Moreover leakages of couplings and fittings must be then very slight because of the small flow rates. 


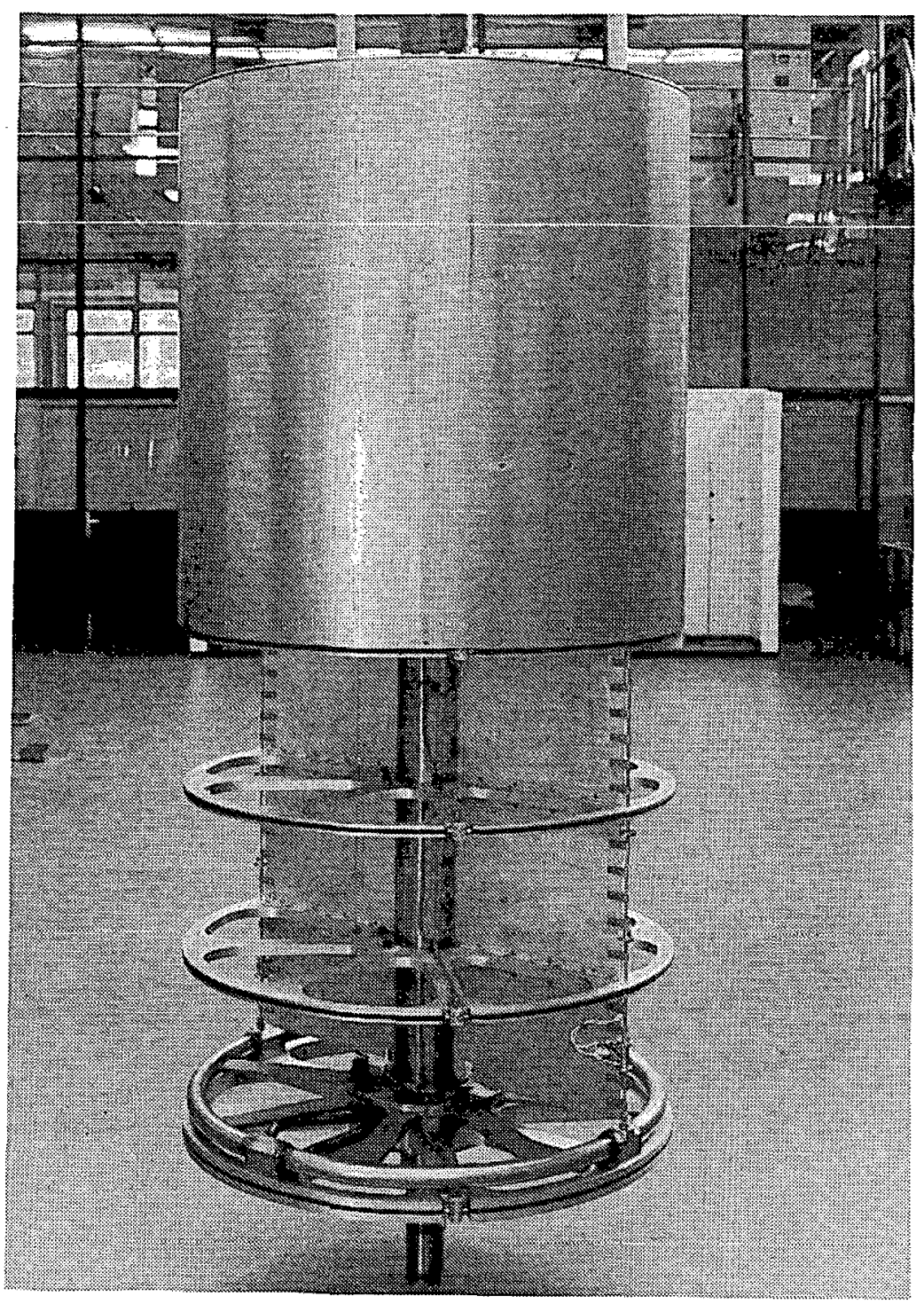

Figure 4.2: The rotor. 


\section{1 .2 Critical speed and balancing of the rotor}

According to the VDI 2060 specification for electric generators the critical speed of a rotor should be $50 \%$ above the operating speed. An exact solution of the natural frequency of a system with distributed mass and flexibility is not possible in general. An estimation of the first natural mode of vibration has therefore been made, using the Raleigh method, see Den Hartog (1947).

Briefly, a shape is assumed for the first normal elastic curve. For conservation of energy, the potential.energy in the position of maximum deflection must be equal to the kinetic energy when passing through the equilibrium position. Raleigh's method of finding the natural frequency is to calculate these maximum energies, equate them, and solve for the frequency. The rotor is regarded as an axis with bearings at both ends, loaded with point masses. With an outer diameter of $140 \mathrm{~mm}$ of the central shaft the first natural frequency becomes approximately $4000 \mathrm{r} . \mathrm{p} . \mathrm{m}$. This is well above the design speed of $1500 \mathrm{r} . \mathrm{p} . \mathrm{m}$.

Balancing of the rotor is desirable for avoiding strong vibrations of the apparatus. At the same time the loads of the bearings are reduced. The rotor is balanced by Holec Slikkerveer according to the VDI 2060 norm. This 1mplies that the imbalance is less than $6 \times 10^{-3} \mathrm{kgm}$. The rotor is balanced with the test section empty. During operation however the tube is filled with a two-phase mixture, which can only partially be compensated. A rod with a weight equal to half of the weight of a liquid filled section is placed in the dummy section opposite the test section. The maximum imbalance due to the two-phase flow now is $9 \times 10^{-3} \mathrm{kgm}$ when using a $5 \mathrm{~mm}$ diameter test section. During the balancing of the rotor the imbalance of the two-phase flow is simulated by placing a rod (imbalance $60 \times 10^{-3} \mathrm{kgm}$ ) in the dumm section. At $850 \mathrm{r} . \mathrm{p} . \mathrm{m}$. the rotor still runs very smooth and no increase of vibrations is noticeable.

Actual operations of the apparatus have revealed that the amplitude of the vibrations are smaller than $0.04 \mathrm{~mm}$ up to $800 \mathrm{r} . \mathrm{p} . \mathrm{m}$. Only in a small range of 580 to $620 \mathrm{r} \cdot \mathrm{p} \cdot \mathrm{m}$. are much stronger vibrations measured. Because no experiments are carried out in this region no action has been undertaken for reducing them (for example by attaching the protection cylinder on the level of the upper bearing to the floor). 


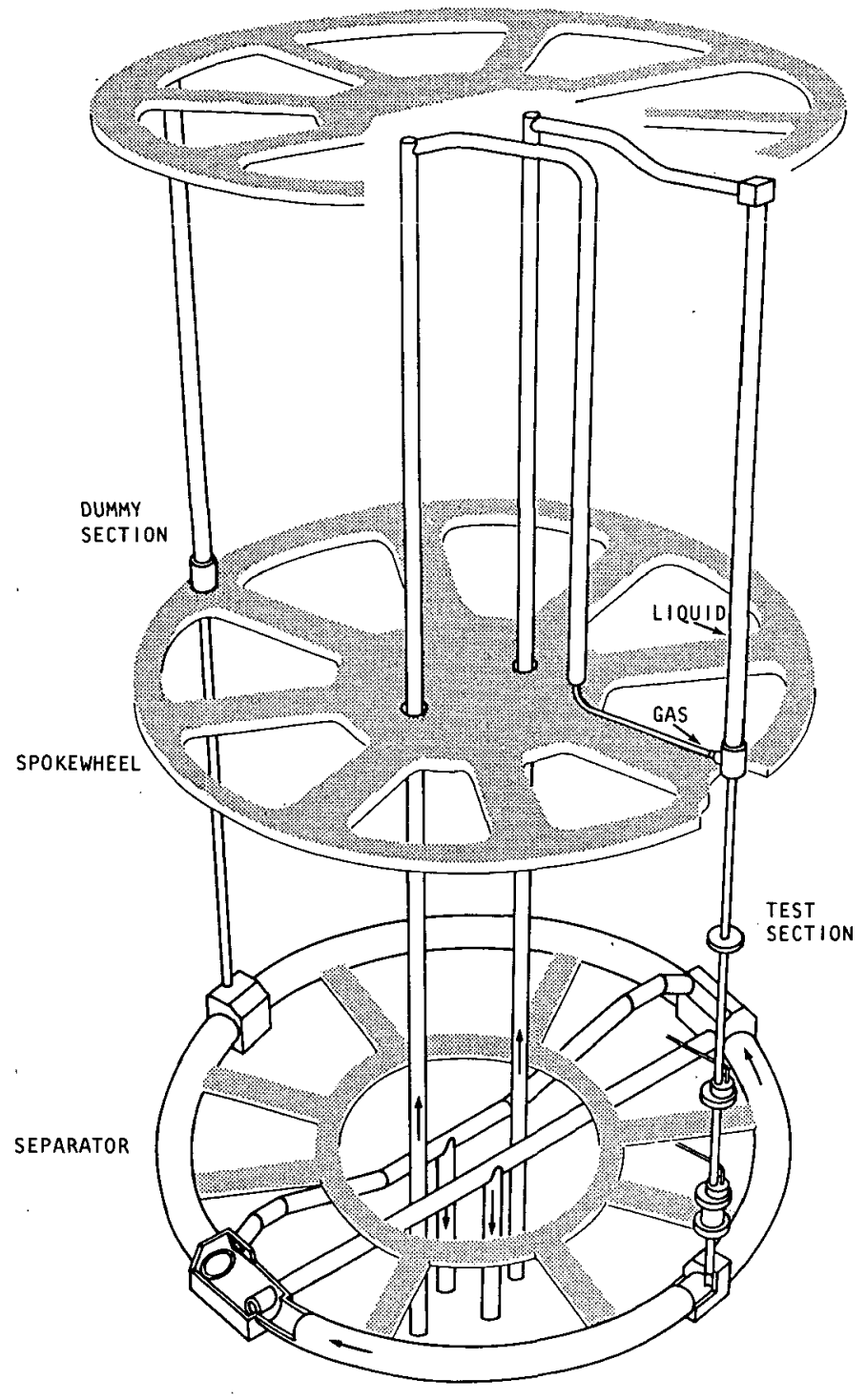

Figure 4.3: A sketch of the rotating flow circuit. 


\subsubsection{The drive and bearings}

The rotor is driven by two $V$-belts underneath the lower bearings. This cholce of drive, rather than direct, chain or gear drive is for space saving reasons and safety aspects. The $V$-belts permit slippage in the event of a bearing or motor fallure, and might prevent destruction of the apparatus. The $V$-belt tension can be adjusted with a pulley mounted on a sliding plate. A 20 kWatt direct current motor is used in combination with a DC generator (Ward Leonard). The motor is rigidly anchored to the floor to minimize vibrations. The rotation speed can be varied continuously between 0 and 1400 r.p.m. To overcome air-friction about 3 kWatt is required and the mechanical seals absorb about $4 \mathrm{kWatt}$. The starting torque however is appreciably higher than the running torque and a powerful motor is therefore applied. A disc with 60 holes at the circumference is attached to the rotor axis. With an infrared source-detector unit and a frequency meter the rotation speed is measured continuously. During operation only small corrections of the speed are necessary, the rotation rate is constant within $0.6 \%$.

The bearings are mounted in the cover and bottom of the protection cylinder, which has a wall thickness of $25 \mathrm{~mm}$. The squared bottom $(1.5 \mathrm{~m} \times 1.5 \mathrm{~m})$ is attached to a frame-work consisting of elght columns, see figure 4.4. In the cover a cylindrical roller bearing is applied, permitting relative axial displacement of the housing and the shaft. The lower bearing is a self-aligning spherical roller bearing which permits angular displacement of the shaft relative to the housing. This is partlculary useful if the rotor has to be lifted out of the protection cylinder for service or modifications.

The radial and axial loads on the bearings are low and the required life of 2000 hours is very short which results in an extremely large safety factor. The reason for selecting a large bearing (inner diameter $=85 \mathrm{~mm}$ ) is to have ample space inside the shaft. A pressure transducer and pressure switch are mounted in the top of the shaft whereas in the lowermost part the liquid and gas Inlet and outlet lines are installed. 


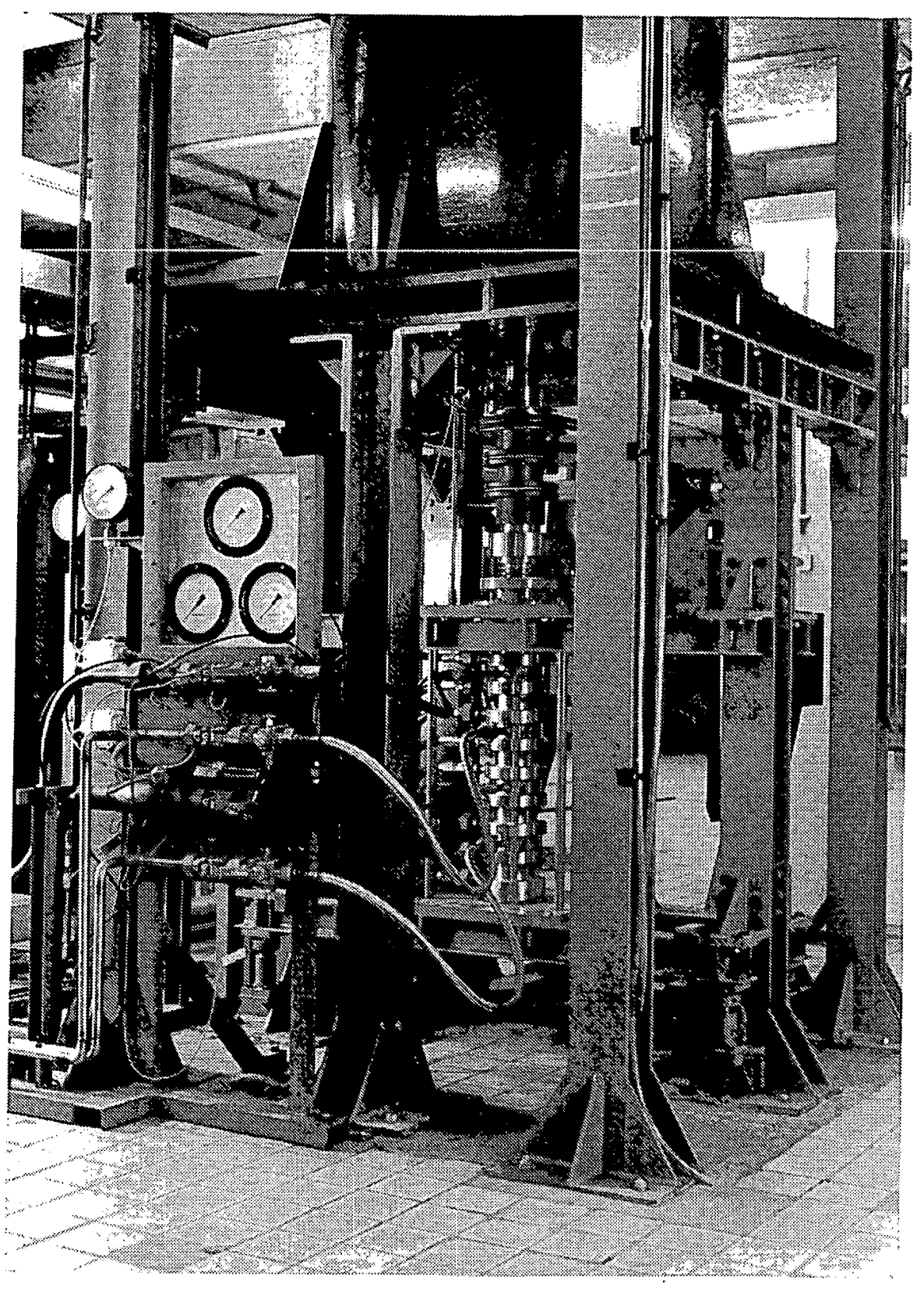

Figure 4.4: A view of the lower part of the centrifuge facility, showing the drive, the flexible couplings and seals. 


\section{1 .4 The seals}

An important part of the set-up is the seal unit to have a "leakage-free" connection between the stationary part and rotating part of the circuit. Five standard double seals (Flexibox, Vlaardingen) are mounted around a stalnless steel hollow shaft (diam $60 \times 50 \mathrm{~mm}$ ). Because of the high system pressure double seals, which require external cooling and lubrication are used. The pressure of the cooling 11 quid (tap water) wust be higher than the pressure to be sealed.

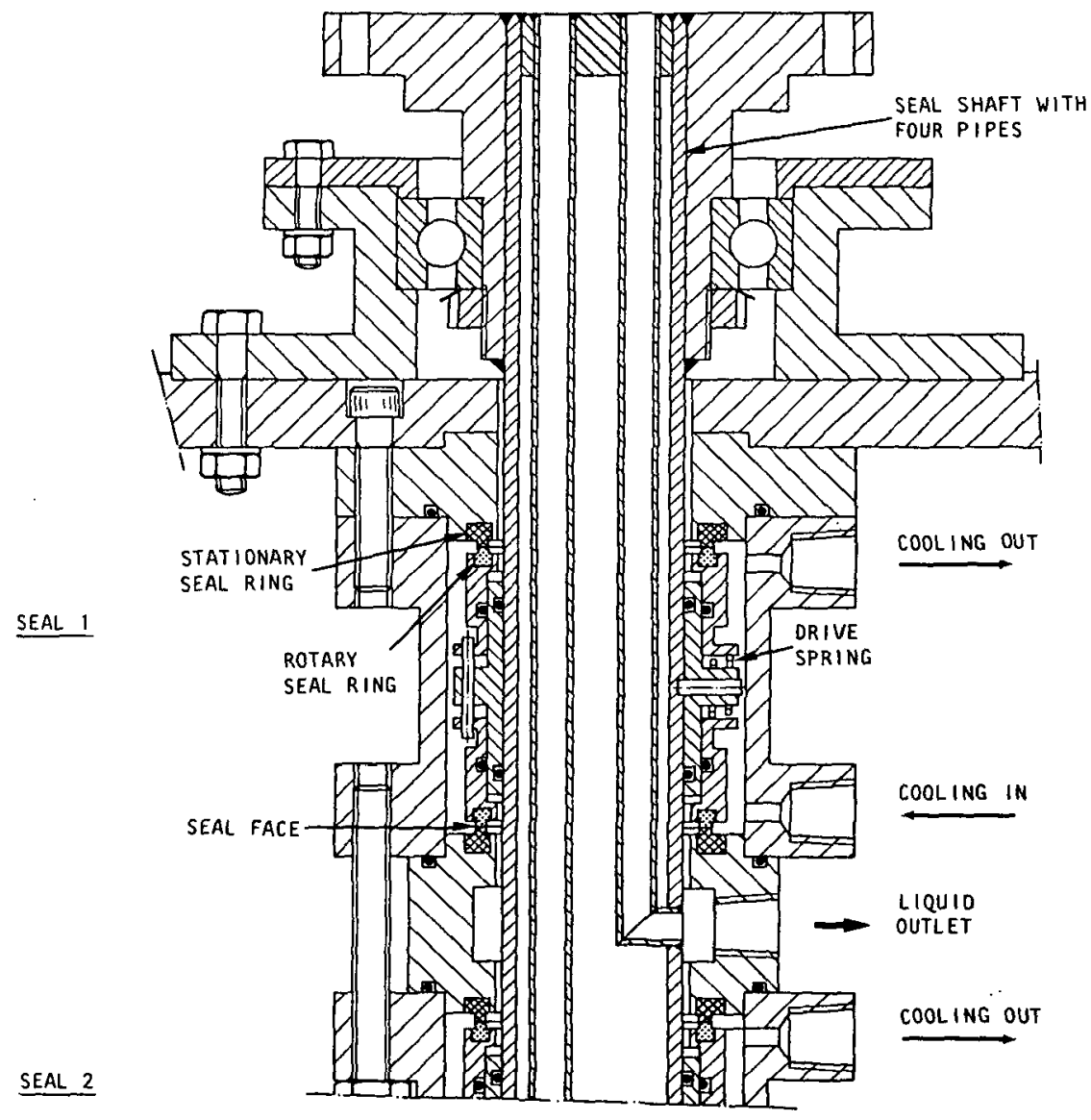

Figure 4.5: Detall of the seal-unit. 
The shaft contains four pipes (diam $14 \times 12 \mathrm{~mm}$ ) for the gas and I1quid flows. One end of a pipe comes out in a hole in the wall of the shaft, the other end is welded to a flange at the top of the shaft. At both sides of the opening in the shaft a mechanical seal is placed. The upper part of the shaft with seals 13 depicted in figure 4.5. The stationary house of the seals is connected by a bush with the appropriate thread port for a pipe connection. At both sides of the shaft bearings are placed. The unit is attached to a frame which can be accurately positioned in all directions for the shaft of the seals to be in line with the central shaft. Small mis-alignments are absorbed by a flexible coupling mounted directly above the seals. In the coupling the fluids flow through stainless steel flexible hoses.

Because the pressure of the cooling liquid is larger than the pressure to be sealed, the only possible leakage is that of cooling water through the seal-face into the circuit. To measure the leakage, the circuit was filled with gas ( $3 \mathrm{MPa}$ ) and after one hour of operation the liquid in the lines is drained. In the beginning the leakage was less than $10 \mathrm{cc} / \mathrm{hour}$ at stand still or rotation. Later on, during the second testing period (test series $A_{12}$ and $A_{14}$ ) the leakage could be up to 1 l/hour which $1 \mathrm{~s} 3 \%$ of the smallest liquid flow rate. Because the leakage took place in the liquid outlet, the flow through the test section was not influenced. A more serlous problem is the increase of the total liquid volume in the circuit. After one hour the separator is almost completely filled with liquid. To overcome this problem liquid is drained during a series of measurements (about 0.51 twice an hour) and the total measuring time is $11 \mathrm{mited}$ to two hours. Just after operation the total amount of liquid in the rotating circuit is measured in order to check the filling degree of the separator. The variation of the level during the experiments is estimated as $\pm 8 \mathrm{~mm}$.

\subsubsection{The slip rings}

The conventional method of transmitting electrical signals from a rotating apparatus is by means of slip rings. Here, for data transmition, silver rings are used which have a relatively low noise level (less than $8 \mu \mathrm{V} / \mathrm{mA}$ at 5000 r.p.m.). Spring loaded silver graphite brushes provide the electrical connection with the stationary terminal board. Four contacts are used for 
the pressure transducer, three for the vold sensor and two for the pressure switch.

The slip rings are mounted on a hollow shaft with bearings on both sides. This unit is attached to a frame which can be fastened to the cover of the protection cylinder. A flexible coupling is used for connecting the shaft of the slip rings with the rotor, see figure 4.6. The unit with the differential pressure transducer and pressure switch contains three valves for venting the tapping lines and flow circuit and one for pressurizing the hoses in the spokewheels.

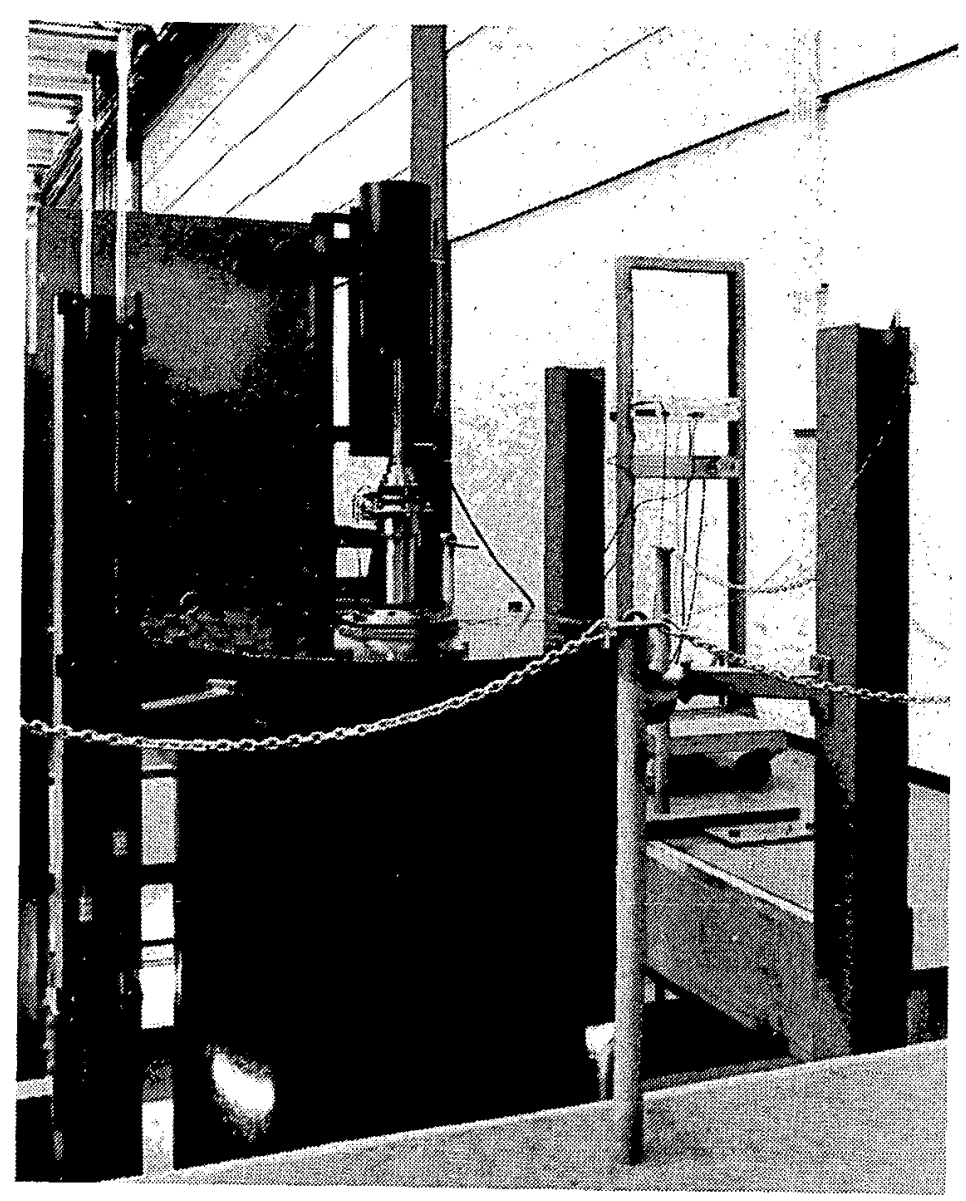

Figure 4.6: A view of the upper part of the centrifuge facility. 


\subsubsection{Description of the circuit and test section}

The main components of the rotating circuit are shown in figure 4.7. A three dimensional sketch is already given in figure 4.3. From the seals 11 quid and gas flow upward in the central lines (diameter $=14 \mathrm{~mm}$ ) to the top of the rotor, where the circuit can be vented. After the radial section, where the pressure increases due to the hydrostatic pressure rise, the liquid flows downward in a $10 \mathrm{~mm}$ diameter tube $1 \mathrm{~m}$ long.

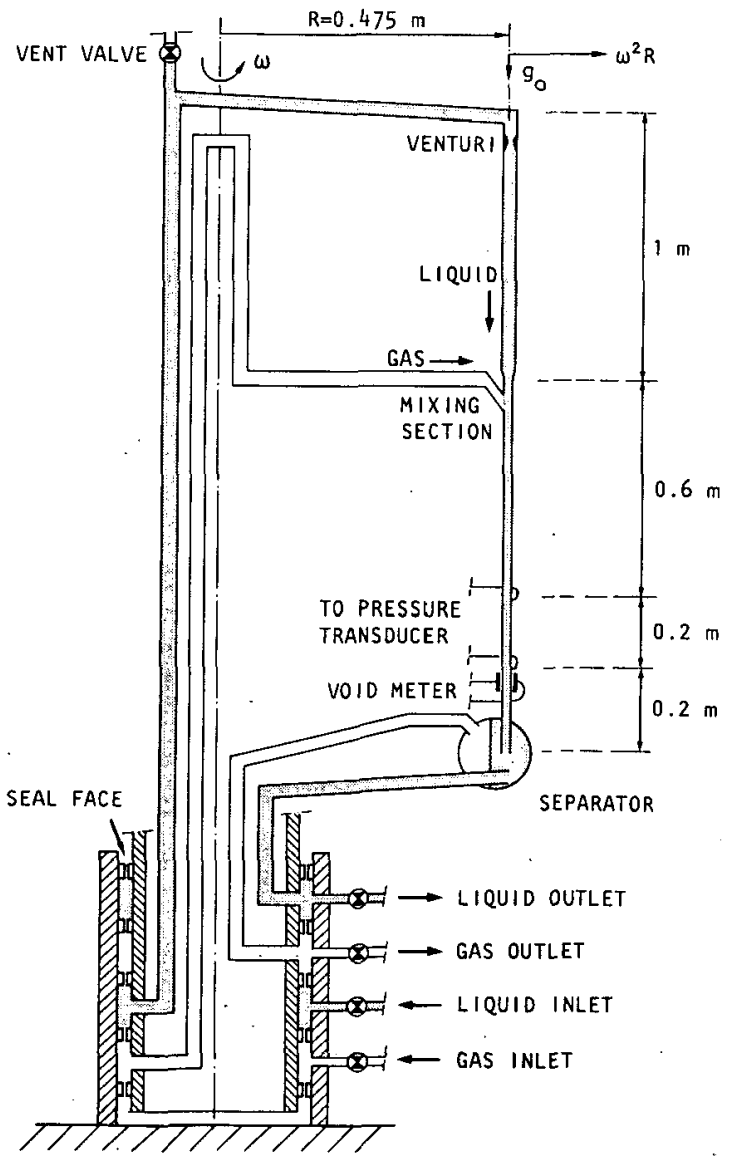

Figure 4.7: Diagram of the rotating loop. 

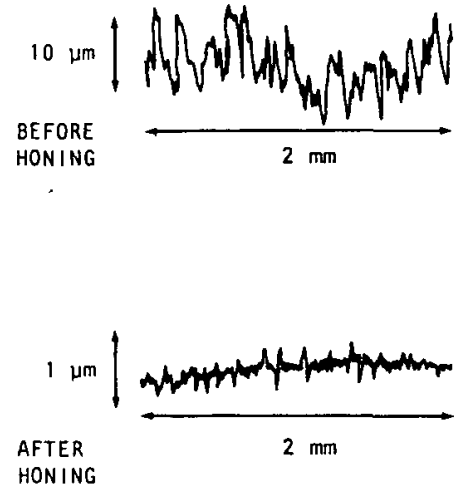

Figure 4.8: Roughness of the wall before and after honing.
At the start of the $10 \mathrm{~mm}$ section the liquid velocity is raised with a venturi (throat diameter $=1.5 \mathrm{~mm}$ ) to prevent back flow of gas. This is only important at low liquid and gas flow rates with a stratified flow pattern. The test section consists of one flanged length, $0.4 \mathrm{~m}$ long, and three flanged lengths, $0.2 \mathrm{~m}$ long, of stainless steel $1 / 4$ inch tube. Because the roughness of the inner wall was relatively large, see figure 4.8, the tube was honed. After honing the inner diameter was $4.90 \pm 0.01 \mathrm{~mm}$ and the roughness of the wall was less than $0.2 \mu \mathrm{m}$. Hence the tube can be regarded as hydrodynamically smooth.

The total length of the $5 \mathrm{~mm}$ test section $1 \mathrm{~s} 1 \mathrm{~m}$ ( = 200 diameters). In the mixing section gas is added to the liquid. The diameter of the gas inlet line $1 \mathrm{~s} 2.2 \mathrm{~mm}$ (see figure 4.9) and the angle with the direction of the flow is $26^{\circ}$. No special demands are imposed on the geometry, only the similarity with the $50 \mathrm{~mm}$ test section is of interest. The flow direction is chosen to be downwards in the direction of the natural gravity, because the influence of a small downward inclination on the stratified intermittent transition is smaller than that of a small upward inclination. The first pressure tap is located after a straight length of $0.6 \mathrm{~m}(* 120 \mathrm{D})$ from the mixing section. The second pressure tap is placed $0.2 \mathrm{~m}$ ( $=40 \mathrm{D})$ downstream.

The sensor for measuring vold fraction is placed Immediately after the second pressure tap. The absolute accuracy of the void fraction measurement is \pm 0.03 . An extensive description of the device is given in chapter 3 .

The entrance of the test section in the separator is shown in figure 4.10. The location of the end of the tube with respect to the centre of the separator is chosen in such a way that with a half fllled separator the test section outlet is $10 \mathrm{~mm}$ below the liquid level. In the ring shaped separator (diameter $=36 \mathrm{~mm}$ ) 11 quid and gas flow in circumferential direction. After a quarter of one revolution the liquid is extracted at the bottom of the tube close to the outerside of the ring and the gas at the top close to the inside of the ring, see pigures 4.3 and 4.7 . 


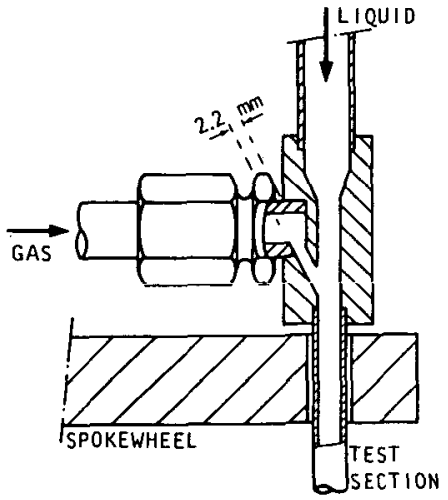

Figure 4.9: Mixing section.

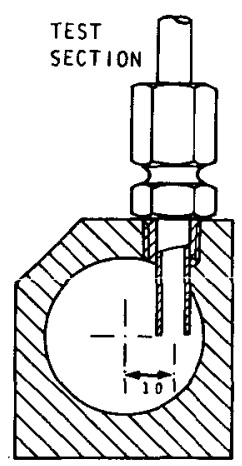

Figure 4.10: Separator inlet.

By equating the time for a rising bubble to reach the liquid surface and the time for the bubble to reach the outlet of the separator, the diameter of the smallest bubble which can be separated from the liquid is estimated. In the half filled case, a flow of $0.1 \mathrm{l} / \mathrm{s}$ and a $\mathrm{g}$-value in the separator of $113 \mathrm{~g}_{0}$, bubbles with a diameter of $6 \mu \mathrm{m}$ are just separated. The separator also serves as a buffer volume (capacity 31 ). The filling degree Increases due to leakage of cooling water through the seal faces into the circuit or by accumulation of gas in the liquid outlet. A decrease of the filling degree can be caused by evaporation or by leakage of the pump and fittings. It was found that leakage of cooling water into the circuit is the main factor by which the level in the separator changes. After the separator the fluids flow via the radial line (diameter $=14 \mathrm{~mm}$ ) and the lines in the rotor shaft (dlameter $=14 \mathrm{~mm}$ ) back to the seals.

The degree of leakage of the circuit is checked before and after a test period of three weeks. To this purpose the circuit is filled with gas (air or helium) at a pressure of $3 \mathrm{MPa}$. From the pressure decrease (corrected for leakage of cooling water into the circuit) in a period of one hour the leakage of gas through couplings and fittings is determined. This appeared to be always less than $1 \%$ of the smallest gas flow. 


\section{2 .2 Filling of the circuit}

Before the test section can be pressurized and rotated, the circuit, with the exception of the gas inlet and gas outlet, must be filled with water. The filling degree of the separator is chosen such that the test section is always below the liquid level (for the $50 \mathrm{~mm}$ test loop the influence of the separator level appeared to be small: see section 5.2.1). A sketch of the filling procedure is given in figure 4.11 .

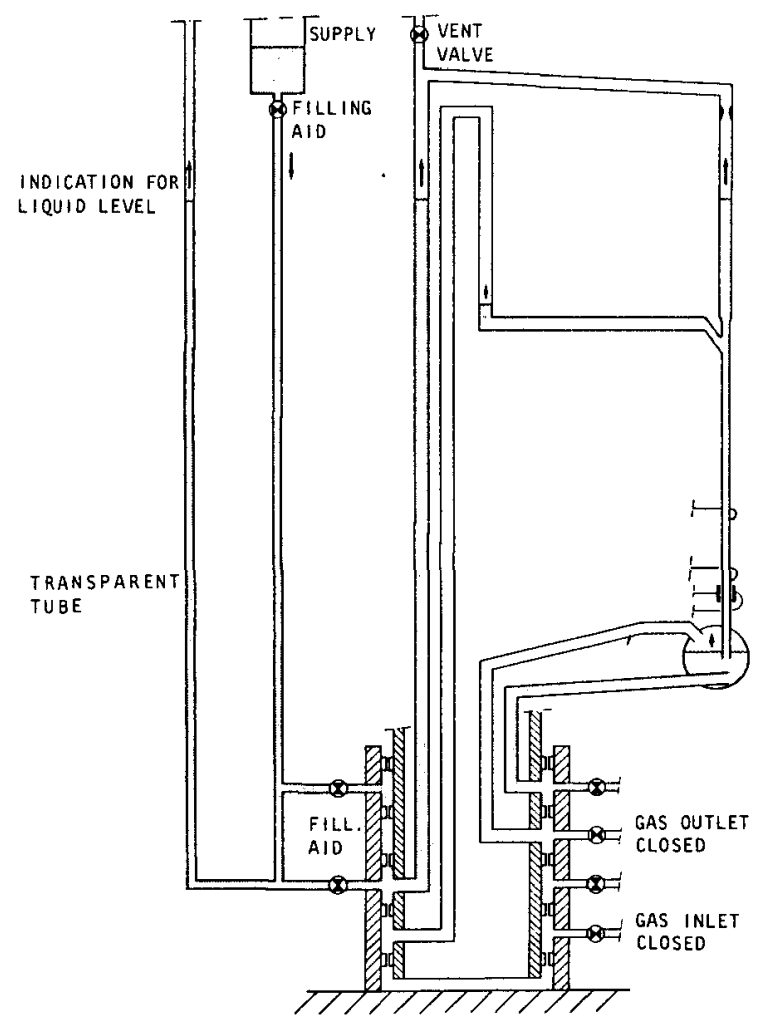

Figure 4.11: Filling of the test section.

Liquid flows from the vessel downwards via the seals into the liquid inlet and liquid outlet. The gas lines are shut of to prevent them filling. During filling the level in the rotor can be observed in a transparent tube outside the rotor. For ease of filling and draining the radial lines run 
smoothly and the liquid outlet is placed at the bottom of the separator. The supply vessel with distilled water is placed $0.3 \mathrm{~m}$ above the vent valves by which the filling degree of the separator can be changed. Beforehand it is checked whether the correct filling degree is obtained by this method. At the start of the experiments the level is $15 \mathrm{~mm}$ above the center of the test section. The tapping lines are vented by means of two valves in the top of the rotor shaft, see figure 4.6 .

\subsection{MEASUREMENT OF PRESSURE DROP}

The two-phase pressure drop is measured over a length of $0.200 \mathrm{~m}$ ( $=40$ diameters). The diameter of the pressure hole in the "bottom" of the flange is $0.3 \mathrm{~mm}$, see figure 4.12 .

The test section was honed after drilling of the holes through which burrs are removed and a sharp-edged form is obtained. With this hole-configuration the error of the pressure drop measurement is small (Shaw, 1959). Before the test section is mounted in the rotor the measured single-phase liquid pressure drop is compared with the calculated value from the Blasius equation, which is sufficlently accurate up to $\operatorname{Re}=10^{5}$ (Schlichting 1979).

$$
\left(\frac{\Delta p}{\Delta L}\right)_{L}=\frac{0.316}{R e^{-1 / 4}} \frac{\rho_{L} U_{L}^{2}}{2 D}
$$

Over the experimental range $2.7 \times 10^{3}<\operatorname{Re}<3 \times 10^{4}$ the difference between the measured and calculated value is less than $4 \%$. The measured values are always higher, exept for the lowest flow velocities. The single-phase pressure drop has also been checked during rotation, see section 4.4.3.

The pressure is transmitted from the taps through liquid-filled lines (1/8 inch tube, $2.5 \mathrm{~m}$ long) to the pressure difference transducer which is mounted in the top of the shaft, see figure 4.6. The unit with the transducer can easily be detached for calibration purposes. The instrument amplifier of the transducer is mounted in the shaft of the slip rings. The transducer (Druck PDCR/120/WL) uses a four arm strain gauge bridge, diffused on a silicon diaphragm. The specifications are: range $35 \mathrm{kPa}$, accuracy $0.1 \%$ of the maximum scale value, natural frequency $10 \mathrm{kHz}, \max$ pressure $7.5 \mathrm{MPa}$. 


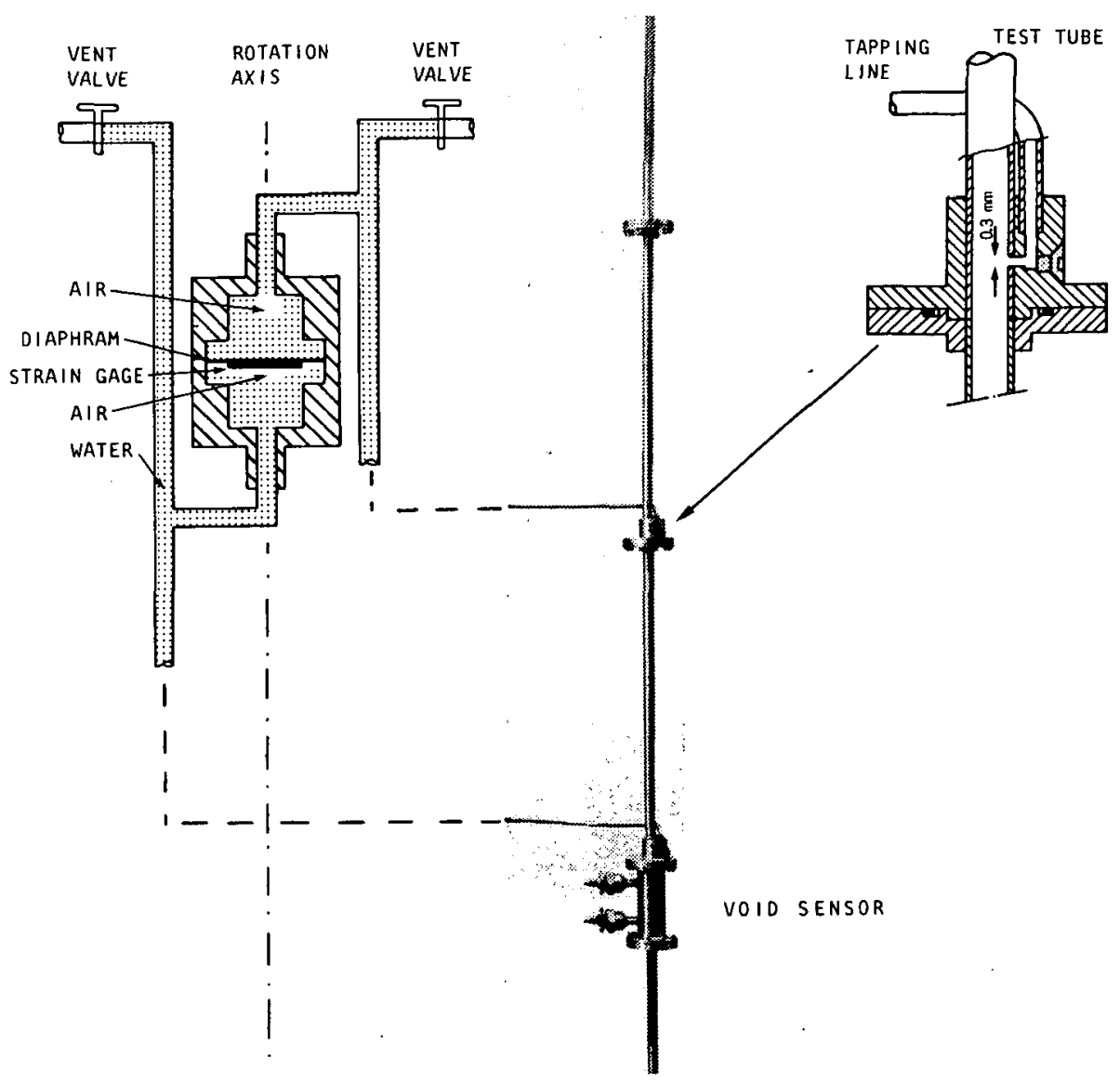

Figure 4.12: A photo of the test section with details of the pressure drop measurement.

According to the specifications the device can be used up to differential pressures of $210 \mathrm{kPa}$ with negligible change in calibration. This offers the possibility of using only one transducer for a wide range and a damping device is not necessary (actual averaged pressure drops lie between $0.3 \mathrm{kPa}$ and $50 \mathrm{kPa}$ and the fluctuations between $-40 \mathrm{kPa}$ and $120 \mathrm{kPa}$ ). Calibrations are performed with a water micromanometer and a mercury micromanometer at four ranges: $-40 \mathrm{kPa}, 2.5 \mathrm{kPa}, 12 \mathrm{kPa}$ and $80 \mathrm{kPa}$. The sensitivities for the different ranges agree within $\pm 1 \%$ and the combined hysteresis and non-linearity is less than 0.28 of the maximum scale values. Hence between 0.5 and $100 \mathrm{kPa}$ the transducer can be used with an accuracy of $2 \%$ of the 
measured value. The influence of rotation of the transducer was tested experimentally. The device was mounted on the shaft of a variable-speed motor. Slip rings were used for electrical operation. The sensitivity is not affected $(<0.1 \%)$, only a drift in the offset directly proportional to $\omega^{2}$ occurs $(0.3 \mathrm{kPa}$ at $1000 \mathrm{r} . \mathrm{p} . \mathrm{m}$.$) .$

The tapping lines are vented with the help of two valves mounted directly above the transducer, see figure 4.6. The transducer itself is not completely free of gas. At both sides of the diaphragm about 2 cc gas is enclosed. By pressurizing the system the volume of gas decreases considerably. Moreover a certain amount of gas dissolves in the surrounding 11quid. At $3 \mathrm{MPa} 1 \mathrm{cc}$ water saturated with helium contains $0.25 \mathrm{cc}$ (at $0^{\circ} \mathrm{C}$ and $0.1 \mathrm{MPa}$ ) gas (with the help of ammonia gas, the sollubility being much higher than that of air or helium, the transducer could have been completely filled with liquid). The change in measured pressure drop due to changes in the liquid level in the transducer
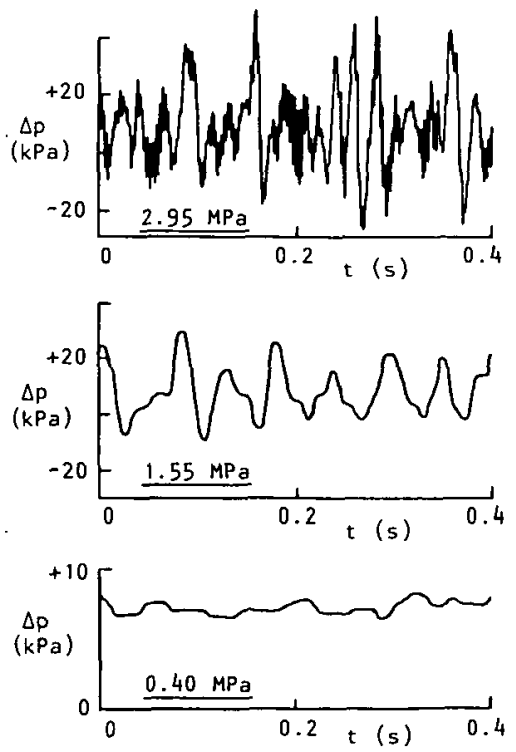

Figure 4.13: Fluctuations of the pressure drop signal. are negligible. For the scaling test $B$ the pressure at the transducer (equal to the difference of the operating pressure and the pressure drop $\rho_{L} \omega^{2} \mathrm{R}^{2} / 2$ in the radial part of the rotor) is low, $0.3 \mathrm{MPa}$. Hence the fluctuations of the volume occupied by the gas can be considerable ( $\pm 10 \%)$, leading to a so-called "pumping action". However as shown below the averaged pressure drop is hardly affected. Figure 4.13 shows the pressure fluctuations for the condition $a_{24}$ at three different system pressures: helium at $2.95 \mathrm{MPa}$ (test series $A_{7}$ ), helium/14\% air at $1.55 \mathrm{MPa}$ (test series $A_{8}$ ) and air at $0.40 \mathrm{MPa}$ (test series $A_{9}$ ), see Appendix I table I-2 and table I-6.

Only the Euler number, which has less influence on the flow, is different for 
the three runs. Although the fluctuations in figure $4.13 \mathrm{c}$ are a factor of 36 smaller than in figure $4.13 a$, the averaged pressure drop differs only $7 \%$. This result justifies the application of damping devices for measuring mean pressure drop (Hewltt 1978, Spedding 1982, Akagawa 1971). The influence of gas inclusions and damping of the pressure fluctuations by needle valves, is also tested with the $50 \mathrm{~mm}$ test loop, see section 5.1 .2 . The time-varying pressure drop signals recorded at varlous days show small differences due to small differences in filling of the transducer. But always the reproducibllity of the mean pressure drop is excellent: $\pm 5 \%$. The reproducibility refers to the whole test series and denotes the maximum observed deviation. For the scaling test $B$ the reproducibility is worse, $\pm 8 \%$ (at the two lowest liquid velocities even $\pm 15 \%$ ), due to the large uncertainty in the temperature.
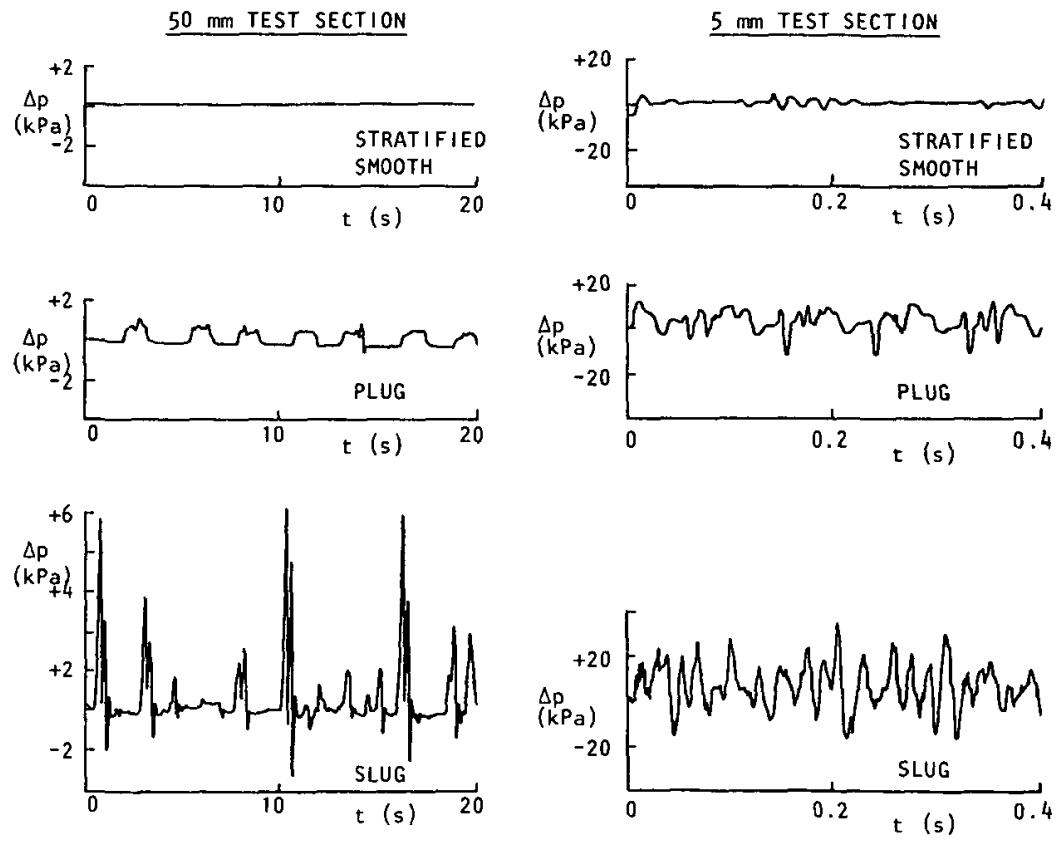

Figure 4.14: Fluctuations in the pressure drop signal for the conditions $a_{21}$ (top), $a_{22}$ and $a_{24}$ of the test series $A_{1}(50 \mathrm{~mm})$ and $A_{7}(5 \mathrm{~mm})$. 
Differential pressure fluctuations are often used for flow pattern identification (Akagawa 1971, Matsui 1984, v.d. Geld 1985). Also in the present work, in cases where no vold meter was avallable, the flow pattern was deduced from the fluctuating pressure signal. For accurate measurements the transducer and tapping lines should be free of gas and the frequency response of the measuring system must be known. Nevertheless from the shape and magnitude of the fluctuations a reasonable estimate of the flou pattern is possible. Figure 4.14 shows some recorded signals of the $5 \mathrm{~mm}$ as well as the $50 \mathrm{~mm}$ test section. The distinction between stratified flow (no or small fluctuations) and plug or slug flow is evident in these cases. At higher flow rates however the signals of plug, slug and semi-annular are very alike. More refined signal analyzing methods are required.

In general the total pressure loss $\Delta p_{t}=p_{1}-p_{2}$ between the pressure taps 1 and 2 (see figure 4.15) is the sum of a frictional term $\Delta p_{f}$, an acceleration term $\Delta \mathrm{p}_{\mathrm{a}}$ (due to changes in kinetic energy) and a gravitational term $\Delta p_{e}$ (because of changes in potential energy):

$$
\Delta p_{t}=\Delta p_{f}+\Delta p_{a}+\Delta p_{e}
$$

For flows without mass transfer between the phases such as are being considered here acceleration effects are small. Even at the lowest system pressure (test series $\mathrm{A}_{9}$ : air $0.40 \mathrm{MPa}$ ) the acceleration pressure loss is less than $1 \%$ of the frictional pressure loss.

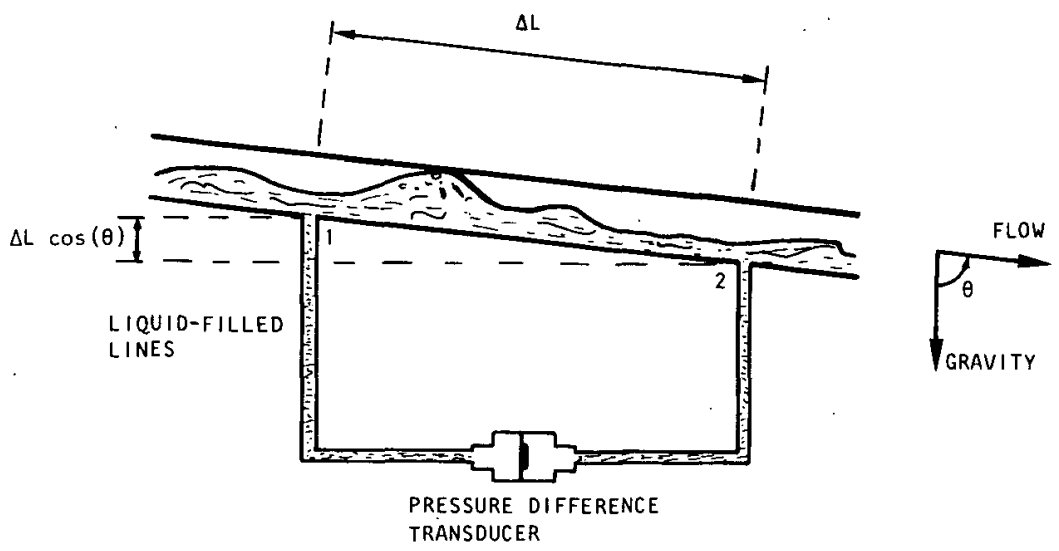

Figure 4.15: A sketch of the pressure drop measurement. 
The pressure difference due to elevation changes is:

$$
\Delta p_{e}=-\left(\alpha p_{G}+(1-\alpha) \rho_{L}\right) g \Delta L \cos (\theta)
$$

where $\theta$ is the angle between the direction of the flow and the direction of the effective gravitational acceleration gravity $\left(\cos \theta=g_{0} / g\right), \Delta L$ is the distance between the pressure taps.

Because the pressure taps are located at different "heights" with respect to the direction of the effective gravitational acceleration the accompaning hydrostatic pressure difference must be added to the total pressure loss to obtain the measured pressure difference $\Delta \mathrm{P}_{\mathrm{m}}$ :

$$
\Delta p_{m}=\Delta p_{f}-\left(\alpha \rho_{G}+(1-\alpha) \rho_{L}\right) B \Delta L \cos (\theta)+\rho_{L} g \Delta L \cos (\theta)
$$

Using $\rho_{G} \ll \rho_{L}$, the frictional pressure loss becomes:

$$
\Delta p_{f}=\Delta p_{m}-\alpha p_{L} g \Delta L \cos (\theta)
$$

The error in the measured pressure loss due to the instrument accuracy is 2\%. The error in the gravitational term is caused by the error in the void measurement ( \pm 0.03 absolute units) and the error in $\Delta \operatorname{Lcos}(\theta)$, due to a deviation of the flow direction from the vertical. The rotor shaft is carefully brought in line with the direction of the natural gravity with the help of a precision levelling instrument. What remains is the orientation of the test section with respect to the rotor shaft. The tolerance in the distance between the pressure hole and the shaft is $\pm 0.1 \mathrm{~mm}$ and the corresponding error in $g \Delta \operatorname{Lcos}(\theta)$ is $\pm 11 \%$.

\begin{tabular}{|lll|lll|lll|lll|}
\hline $\begin{array}{l}\text { test } \\
\text { cond }\end{array}$ & FP & Ratio & $\begin{array}{l}\text { test } \\
\text { cond }\end{array}$ & FP & Ratio & $\begin{array}{l}\text { test } \\
\text { cond }\end{array}$ & FP & Ratio & test & FP & Ratio \\
\hline$a_{11}$ & SS & 0.80 & $a_{21}$ & SS & 0.30 & $a_{31}$ & PL & 0.13 & $a_{41}$ & PL & 0.01 \\
$a_{12}$ & SS & 0.46 & $a_{22}$ & PL & 0.26 & $a_{32}$ & PL & 0.14 & $a_{42}$ & PL & 0.02 \\
$a_{14}$ & SW & 0.34 & $a_{24}$ & SL & 0.16 & $a_{33}$ & PS & 0.12 & $a_{43}$ & PL & 0.02 \\
$a_{15}$ & SA & 0.12 & $a_{25}$ & SA & 0.06 & $a_{34}$ & PL & 0.10 & $a_{44}$ & SL & 0.02 \\
\hline
\end{tabular}

Table 4.1: Ratio of the gravitational term to the measured pressure drop. 
The gravitational term can be a considerable part of the measured pressure drop, see table 4.1. The error in the frictional component, determined by substracting the gravitational term from the measured pressure drop can therefore be large too at low fluid velocities: $90 \%$ at $a_{12}, 15 \%$ at $a_{12}$ and $10 \%$ at $a_{21}$.

\subsection{ADJUSTMENT OF MAIN PARAMETERS}

The flow sheet for circulating liquid and gas is given in figure 4.16 .

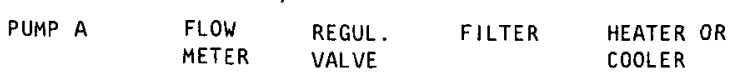

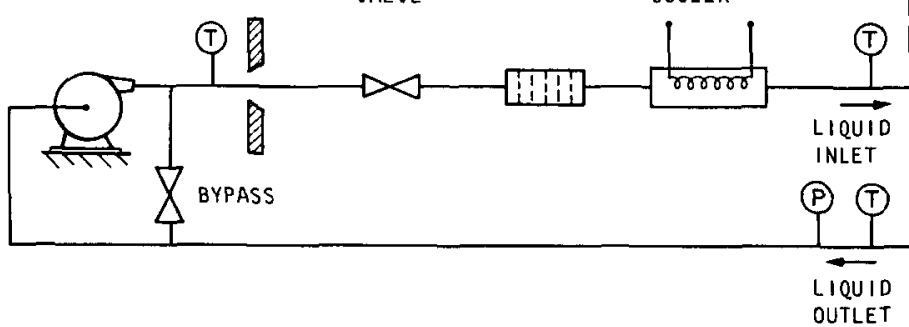

$\begin{array}{ll}\text { PRESSURE } & \text { AIR SUPPLY } \\ \text { CONTROL } & 15 \mathrm{MPa}\end{array}$

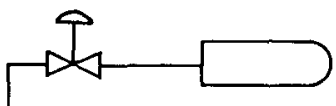

LOON $1.3 \mathrm{~m}^{3}$

FLOW REGUL.

ELECTRIC

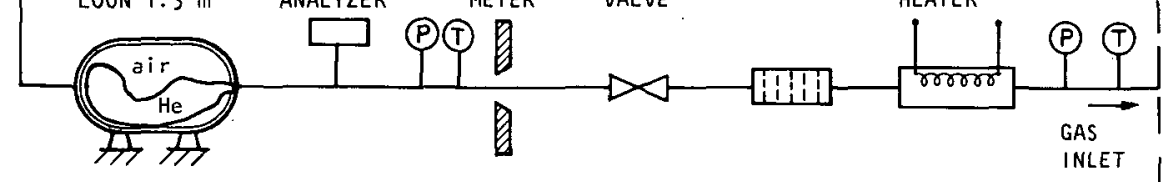

$\mathrm{R}$

0

A

$\mathrm{T}$

OUTLET

$\mathrm{N}$

G

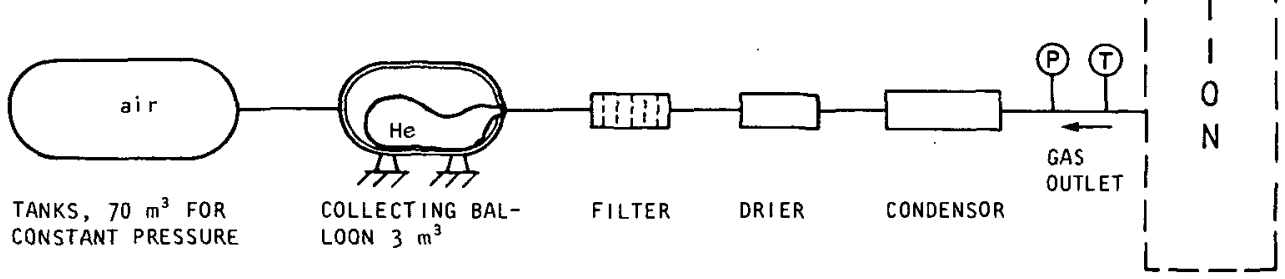

Figure 4.16: Flow sheet for circulating liquid and gas. 


\subsubsection{The liquid circuit}

An eight stage centrifugal pump driven by a variable speed motor is used for circulating liquid at a maximum operating pressure of $4 \mathrm{MPa}$. The available head at the required flow rates $(0.011 / \mathrm{s}-0.1 \mathrm{l} / \mathrm{s})$ is about $1.8 \mathrm{MPa}$. The mass flow rate is measured with integral orifices (squared edge orifices especially for small flows). The whole experimental region is covered with three orifices plates, placed in parallel lines. The flow is adjusted by means of three regulating valves downstream of the flow meters and a bypass over the pump. Before the liquid enters the seal unit it is filtered and brought to the desired temperature with the help of a tubular heat exchanger. The temperature is measured with thermocouples (instrument error $\pm 0.5^{\circ} \mathrm{C}$ ) in front of the flow meter and in the liquid inlet and outlet. The total content of the circuit is approximately 201 . The pressure in the liquid outlet is measured with a pressure gauge (error $\pm 20 \mathrm{kPa}$ ).

During rotation the pressure difference between liquid outlet and gas outlet must be equal to the hydrostatic pressure drop in the radial part of the rotor. If for any reason the liquid outlet is filled with gas, then the pressure balance is disturbed and the rotating circuit must be refilled.

\subsubsection{The gas circuit}

A facility has been built for blowing any gas or gas mixture through the test section at a maximum operating pressure of $4 \mathrm{MPa}$. The gas to be circulated is stored in a balloon $\left(1.3 \mathrm{~m}^{3}\right)$ which is enclosed by a pressure vessel. The air pressure in the vessel is kept at $4 \mathrm{MPa}$ by means of a control unit feeded by the high pressure (15 MPa) utility net of the laboratory. By turning on the regulating valve, gas flows from the supply balloon via the seals and the rotating test section to the collecting balloon $\left(3 \mathrm{~m}^{3}\right)$ which is also enclosed by a pressure vessel. The pressure is kept constant by means of two tanks (total capacity $70 \mathrm{~m}^{3}$ ) from the medium pressure utility net, see figure 4.16.

In each vessel two pressure switches are mounted, registrating when the pressure difference between the gas in the balloon and the air in the vessel exceeds the treshold level for empty $(-8 \mathrm{kPa})$ and full $(+8 \mathrm{kPa})$. If the supply ballon is empty or the collecting balloon is full, the flow is automatically stopped. The filled collecting balloon fits closely in the 
vessel and hence the chance of rupture the balloon is small. The supply balloon on the other hand is smaller than the vessel and therefore two electric discharge valves operated by pressure switches are used for protection. Using air pressure the gas is pumped from the collecting balloon back to the supply balloon and the same amount of gas can be used several times. With a full supply balioon the experiments can be runned continuously for almost two hours at a medium flow rate.

The mass flow rate is, like the liquid flow rate measured by integral orifices (Taylor Instruments). Three orifice plates are placed in parallel lines and exchanging of them is limited. Needle valves, located downstream of the flow meters are used for regulating the flow rate.

Before the gas flows into the test section it is filtered and if necessary heated by electric heating elements ( 5 kWatt). After the test section the gas is drled (with silicagel) and filtered. Temperature (instrument error $\pm 0.5^{\circ} \mathrm{C}$ ) and pressure are measured in front of the flow meter and in the gas inlet and outlet. If helium or a helium/air mixture is used the percentage of air is measured with a gas analyzer. The principle is based on the change of the thermal conductivity with air concentration. A few tests were carried out with pure helium from a high pressure (20 MPa) gas cylinder.

\section{4 .3 General procedure for measurements}

The experiments for the scaling tests $A$ and $B$ are carried out in two perlods each of about three weeks. In the first period only pressure drop is measured. The density ratio, the Euler number and Froude number are varied. In the second perlod the Reynolds number and Froude number are varied and the measurements of the scaling test $B$ are carried out.

Before and after each period the following controls are made:

- calibration of the pressure difference transducer

- calibration of the vold sensor

- calibration of the pressure gauges

- callbration of the gas analyzer

- checking of the flow measurement

- measurement of the leakage of the rotating circuit. 
The leakage of cooling water into the circuit is checked every day. Due to this leakage the specific conductivity of the water increases from $6 \times 10^{-4} 1 / \Omega \mathrm{m}$ at the start up to $40 \times 10^{-4} 1 / \Omega \mathrm{m}$ at the end of the experiment.

To start up the test facility, the following steps are undertaken. First the circuit with the pump and the flow meter is filled with distilled water. Care is taken for the circuit to be completely free of gas ( 10 vent valves are used). Then the cooling system of the seals is started and the leakage is measured (If necessary liquid is drained during the experiments to maintain an acceptable filling degree of the separator). Now the circuit with the test section is filled. The filling degree of the separator is adjusted by means of the position of the supply vessel, see section 4.2.2. Liquid is circulated through the test section to prevent gas from entering the lines upstream of the test section. The system is carefully pressurized via the gas inlet and gas outlet. During pressurizing the rotation is started. After adjusting the correct rotation rate, the circuit is ready for experiments.

Before a two-phase flow is adjusted always the mean value, $p_{L}$, of the pressure signal, with only liquid flowing through the test section is first measured. This value is used as reference value for the two-phase pressure drop, see equation 4.7. The difference of two single-phase pressure levels is compared with the calculated value from the Blasius equation (4.1). The deviation is always less than $\pm 5 \%$.

Then a two-phase flow is adjusted and for at least 30 seconds the averaged values (averaging time $=10$ seconds) of the output signals of the differential pressure transducer, the void meter and the flow meters are measured.

Three recordings are sufficient to obtain a reliable averaged value of the pressure drop and void fraction. The difference between two measurements is always less than 3\% for the pressure drop and less than 0.01 for the vold fraction.

At the start and the end of the run, recordings are made of:

- the pressure and temperature in the gas outlet

- the pressure and temperature in the liquid outlet

- the pressure and temperature in front of the gas flow meter

- the rotation speed. 
The gas analyzer is read off only twice an hour because the drift of the molecular weight of the gas is relative small. All values are written down by hand.

The time-varying void signal and pressure signal are recorded simultaneously for $0.4 \mathrm{sec}$ with a digital oscilloscope (Nicolet 3091 , sample frequency is $10 \mathrm{kHz}$ ). The flow pattern is deduced from the void signal, see section 3.5 .

Only sporadically and then for a very short time, the liquid flow is stopped to measure the threshold value of the pressure difference transducer. At very small or zero flow gas can enter the lines in front of the venturi by which the pressure balance is disturbed and the set-up must be restarted. Especially in the first testing period, when the venturi was not built in yet, the experiments were regulary interrupted by this.

The averaged output signal $p_{L}$ of the transducer with liquid flowing alone through the test section is used, together with the calculated single-phase liquid pressure difference $\Delta p_{L}$, the capacitance $c_{1 i q}$ of the liquid filled sensor and the capacitance $C_{\text {gas }}$ of the empty sensor, for calculating the void fraction $\alpha$ and friction multiplier $\phi_{L}^{2}$ :

$$
\begin{aligned}
\alpha & =\frac{C_{\text {mix }}-C_{1 i q}}{C_{\text {gas }}-C_{1 i q}} \\
\phi_{L}^{2} & =\frac{p_{t p}-p_{L}-\alpha \rho_{L} g \Delta L \cos (\theta)}{\Delta p_{L}}+1
\end{aligned}
$$

where $C_{m i x}$ is the capacitance of the two-phase mixture and $p_{t p}$ is the averaged pressure signal during the two-phase flow.

The experiments were repeated on different days and in different weeks to check whether the measurements could be reproduced. For the scaling test $\mathrm{A}$ the reproducibility of the friction multiplier is $\pm 5 \%$ and the reproducibility of the void fraction measurement is \pm 0.02 . In 12 out of 17 adjusted flows the reproducibility is even better: $\pm 2 \%$.

Due to the relative large uncertainty in the temperature, the reproducibility of the measurements of the scaling test $B$ is worse: at the 
four highest liquid flow rates $\pm 8 \%$ and at the two lowest velocities $\pm 15 \%$ and the reproducibility of the void fraction is \pm 0.03 .

In Appendix I and II the fluid velocities, dimensionless groups, fluid properties, system parameters and experimental results are listed for the two scaling tests.

\subsubsection{Accuracy of main parameters and dimensionless groups}

In this section the tolerances of the adjusted parameters (fluid velocities and dimensionless groups) are given in detall for the $5 \mathrm{~mm}$ test section. For ease of comparison the tolerances, concerning the 5, 50 and 100 $\mathrm{mm}$ test section are put together in table 4.2.

The word tolerance is used here to describe the likely accuracy, not the maximum possible absolute error. If the errors are normally distributed the tolerance is defined as twice the standard deviation, corresponding with 95\% confidence limits, i.e. in 95 cases out of 100 on average, the true value will lie within these limits. Usually only one measurement is carried out and an estimation of the standard deviation is required. Here the standard deviation of a single measurement is chosen to be half the maximum absolute error. The tolerance of a quantity, depending on various parameters with individual error is determined as the square-root of the sum of the squares of each individual tolerance. In repeated measurement, some of the sources of errors considered in calculation of the total tolerance will give rise to errors which vary (random errors) from one measurement of flow to another, while others will give rise to persistent (systematic) errors, which affect all measurements in the same way. The reproducibility of the results is governed by the magnitude of the random errors, while the accuracy of the system is set by the possible systematic errors. Most errors met with here are of the random type. Systematic errors, arrising from the use of flow. coefficients, correction factors and non-linear calibration curves are small.

For comparing the original and model flow prescribed values of the density, the temperature, the gravity and the fluid velocities must be adjusted in the test sections. These values are called the ideal values. 
If during the two-phase measurements the quantities mentioned above deviate considerably from the ideal values then the parameters are readjusted according to the scheme of figure 4.17 and the measurement is repeated.

Despite adjustment of the parameters the most important source of error is the deviation of the readed values from the ideal ones.

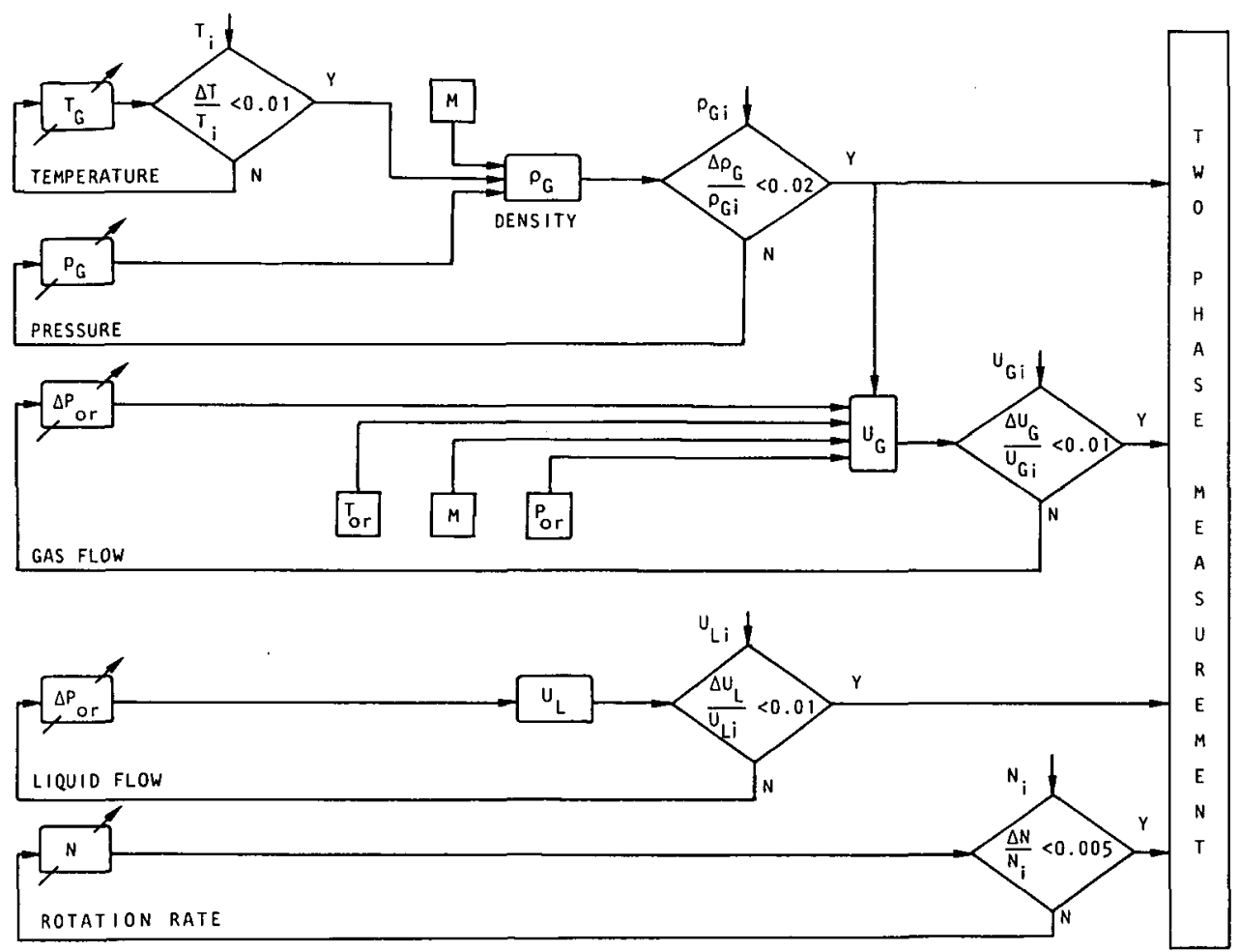

F1gure 4.17: Adjustment of the main parameters. $P_{o r}$ and $T$ or are the pressure and temperature in front of the orifice and $\Delta P_{\text {or }}$ is the pressure drop over the orifice, 1 indicates ideal value.

The parameters from which the dimensionless groups and velocity ratio are composed are:

liquid: density, viscosity, surface tension and superficial velocity gas: density, viscosity and superficial velocity

system: diameter, gravity and operating pressure. 
The operating pressure is used for compensating the change of the gas density due to a drift of the molecular weight of the gas. The viscosity of the gas is not considered because of the relative low importance of it in two-phase flow. The diameter of the tube is measured with an accuracy of $0.3 \%$. The error in the effective gravity including of the variation of the $g$ over the tube radius is $1.5 \%$.

For pure liquids the density, viscosity and surface tension are mainly dependent on the temperature. For the scaling test $A$ the liquid temperature $\left(20^{\circ} \mathrm{C}\right)$ is constant within $2^{\circ} \mathrm{C}$. The scaling test $\mathrm{B}$ is carried out at $55^{\circ} \mathrm{C}$ and the error is much larger: $\pm 5^{\circ} \mathrm{C}$. The relative errors in the density, surface tension and viscosity now are $0.1 \%, 0.5 \%$ and $5 \%$ for test $A$ and $0.5 \%, 2.5 \%$ and $10 \%$ for test $B$.

The gas density is calculated from the measured values of the absolute pressure $\mathrm{p}_{G}$ in the gas outlet, the temperature $T$ in the gas outlet and the molecular weight $M$ :

$$
\rho_{G}=\frac{p_{G} M}{Z R T}
$$

The compressibility factor $Z$ takes account of the deviation of real gases from the ideal gas law. At $4 \mathrm{MPa}, Z=1.02$ for helium and $Z=0.99$ for air. The relative error $\triangle M / M$ is $0.5 \%$ for helium mixtures up to $5 \%$ air by volume. The error in the outlet pressure is due to:

- the instrument error $( \pm 0.5 \%)$

- the drift of the absolute pressure (maximum $\pm 0.5 \%$ ), associated with the variation of the liquid level in the separator (maximum $\pm 8 \mathrm{~mm}$ )

- the deviation of the readed pressure from the ideal value (error $\pm 1 \%$ ). The resulting tolerance in $\mathrm{p}_{G}$ is $\sqrt{ }\left(0.5^{2}+0.5^{2}+1^{2}\right)=1.2 \%$.

The temperature of the gas is constant within $3^{\circ} \mathrm{C}$ at room temperature and within $12^{\circ} \mathrm{C}$ at an operating temperature of $55^{\circ} \mathrm{C}$.

The relative error in the gas density is now: $\sqrt{ }\left(1.2^{2}+0.5^{2}+1^{2}\right)=1.6 \%$ for the scaling test $\mathrm{A}$ and $\sqrt{ }\left(1.2^{2}+0.5^{2}+3.6^{2}\right)=3.8 \%$ for the test $\mathrm{B}$.

The superficial velocity $U$ is calculated from the pressure drop $\Delta \mathrm{P}$ or over the orifice, the density $\rho$ or in front of the orifice, the density $\rho$ of the fluid in the outlet of the test section and the diameter of the tube: 
$U=\frac{4 K \Delta P_{o r}^{1 / 2} \rho_{o r}^{1 / 2}}{\pi \rho D^{2}}$

The error in the flow coefficient $K$, which is determined by calibration, is less than $0.9 \%$ (Dijkshoorn 1985). The error in $\Delta P_{\text {or }}$ is due to the instrument error and the deviation of the readed value from the ideal one.

For the liquid the error in $\Delta P_{\text {or }}$ is $1.4 \%$ and the tolerance in the velocity measurement is: $\sqrt{ }\left(0.9^{2}+1 / 4 \times 1.4^{2}+1 / 4 \times 0.1^{2}+4 \times 0.3^{2}\right)=1.3 \%$ for test $\mathrm{A}$ and $1.5 \%$ for test $B$.

\begin{tabular}{|c|c|c|c|c|c|c|c|c|}
\hline & & \multicolumn{7}{|c|}{ Tolerance (\$) } \\
\hline & & $\mathrm{U}_{\mathrm{L}}$ & $\mathrm{U}_{\mathrm{G}}$ & $\rho_{L}$ & $P_{G}$ & Re & $\mathrm{Fr}$ & We \\
\hline \multirow{2}{*}{$\begin{array}{l}\text { scaling } \\
\text { test A }\end{array}$} & $5 \mathrm{~mm}$ & \multirow{2}{*}{$\begin{array}{l}1.3 \\
1.2 \\
2.5\end{array}$} & 1.7 & 0.1 & 1.5 & 5.2 & 3.0 & 2.7 \\
\hline & $50 \mathrm{~mm}$ & & 1.6 & 0.3 & 1.3 & 4.2 & 2.4 & 2.8 \\
\hline \multirow{3}{*}{$\begin{array}{l}\text { scaling } \\
\text { test } B\end{array}$} & $\mathrm{~mm}$ & \multirow{3}{*}{$\left.\mid \begin{array}{l}1.5 \\
1.2 \\
2.5\end{array}\right\}$} & 2.7 & 0.3 & 3.8 & 10.0 & 3.5 & 3.9 \\
\hline & $50 \mathrm{~mm}$ & & 1.6 & 0.1 & 1.3 & 4.2 & 2.4 & 2.4 \\
\hline & $100 \mathrm{~mm}$ & & 3 & 0.5 & 5 & 8 & 4 & 7 \\
\hline
\end{tabular}

Table 4.2: Errors of main parameters and dimensionless groups of the 5, 50 and $100 \mathrm{~mm}$ test sections. For the $50 \mathrm{~mm}$ section two errors are given for the liquid velocity, the upper for $U_{L}<0.5 \mathrm{~m} / \mathrm{s}$, the lower for $U_{L}>0.5 \mathrm{~m} / \mathrm{s}$.

Before a gas flow is adjusted the ideal value of the pressure drop over the integral orifice is calculated from:

- the actual pressure and temperature in front of the orifice

- the measured molecular weight

- the actual values of the pressure and temperature in the gas outlet. Hence for the error in $\rho_{\text {or }}$ and $\rho_{G}$ only the instrument errors have to be taken into account $( \pm 0.8 \%)$. Because of the large uncertainty of the temperature for the scaling test. $B$ the error for $\rho_{G}$ is estimated as $\pm 2 \%$. The pressure drop over the orifice is adjusted with an accuracy of $2 \%$ for the test $A$ and $3 \%$ for the test $B$. The error in the gas velocity is now: $\sqrt{ }\left(0.9^{2}+1 / 4 \times 2^{2}+1 / 4 \times 0.8^{2}+0.8^{2}+4 \times 0.3^{2}\right)=1.7 \%$ for the scaling test $A$ and $\sqrt{ }\left(0.9^{2}+1 / 4 \times 3^{2}+1 / 4 \times 0.8^{2}+2^{2}+4 \times 0.3^{2}\right)=2.7 \%$ for the test $B$. 


\subsection{CONCLUSIONS}

A centrifuge facility has been built at the Delft University of Technology for scaling horizontal two-phase gas-liquid flows. The test section with an inner diameter of $5 \mathrm{~mm}$ and a length of $200 \mathrm{D}$, rotates around a parallel vertical axis.

The Froude number can be varied very easily by changing the rotation rate. In principle any non-toxic liquid and gas (or gas mixture) can be circulated through the test section at a maximum operating pressure of $4 \mathrm{Mpa}$. Hence a wide range of the gas to liquid density ratio is practicable.

Very accurate measurements of pressure drop and vold fraction are possible up to 1200 times natural gravities. For the scaling test $A$ the reproducibility of the pressure drop is better than $\pm 5 \%$ and that of the vold fraction better than \pm 0.02 . The flow pattern is derived from the timevarying void-signal.

The reproducibility of the experiments in the scaling test $B$ is worse. Because of the high operating temperature the tolerances of the adjusted gas velocity and gas density are large. 


\section{THE 50 AND 100 MM TEST FACILITIES}

In this chapter the two stationary test facilities, for obtaining the large scale data are described. Attention is paid to the accuracy and reproducibility of the measurements. Results of preliminary experiments concerning the influence of the separator level, the inclination angle, developed flow and gas inlet geometry are reported in 5.2 and 5.3.5.

\subsection{DESCRIPTION OF THE 50 MM TEST FACILITY}

\section{1 .1 The test section}

A sketch of the test section is given in figure 5.1. Much attention was paid to the geometrical similarity of the 50 and $5 \mathrm{~mm}$ test section:

- The same length to diameter ratio (200) is used.

- The same inlet geometry is applied. The angle of the gas inlet to the direction of the main flow is $26^{\circ}$. The diameter of the gas inlet is $0.45 \mathrm{D}$ ( $D=$ diam test section).

- The same outlet condition is valid: the liquid level in the separator is always above the entrance of the test section in the separator.

- The same dimensionless distance, $40 \mathrm{D}$, between the pressure taps is used.

- The same type of void sensor is applied, see chapter 3 .

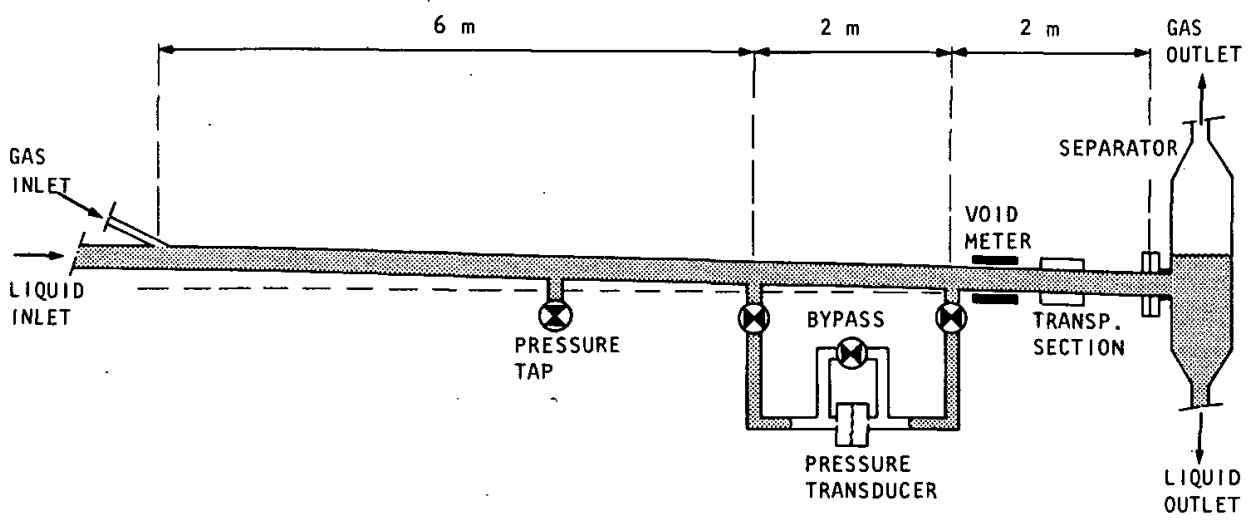

Figure 5.1: The $50 \mathrm{~mm}$ test section. 


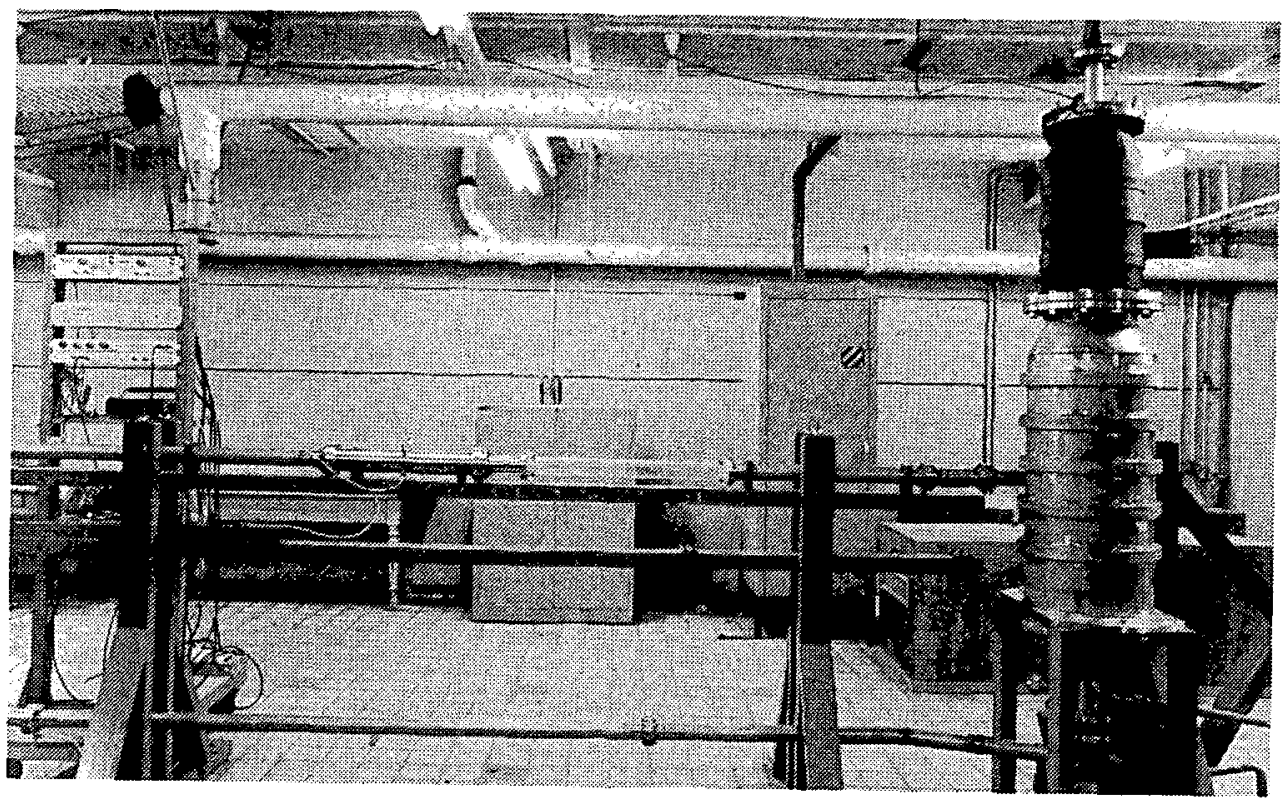

Figure 5.2: Photo of the test section with the separator.

The test section is constructed of four flanged stainless steel sections $2 \mathrm{~m}$ long and three flanged sections $0.65 \mathrm{~m}$ long. The pipe joints are made in such a way that wall discontinuities are absent. The mean inner diameter of the pipe, determined by filling a $2 \mathrm{~m}$ long section with water, is $49.80 \pm 0.05 \mathrm{~mm}$. The roughness of the wall is less than $4 \mu \mathrm{m}$, so that the test section can be regarded as hydrodynamically smooth up to Re $=10^{6}$. The flexible hoses at the entrance of the separator and just before the mixing section, allow the test section to be inclined downwards, the maximum slope being \pm 0.75 degree. The inclination angle is adjusted with the help of a precision levelling instrument and the error in the slope is less than 0.02 degree.

Gas (air) is added to the liquid in the mixing section. After a straight length of $6 \mathrm{~m}$, pressure drop is measured over a distance of $2 \mathrm{~m}$. 
Usually two of the three $0.65 \mathrm{~m}$ long sections just behind the pressure taps are replaced by the vold sensor and a transparent acrylate section for visual observations. However if propanol is used (test series $A_{6}$ ) the void sensor as well as the transparent section have to be left out because the liquid causes large stresses in the acrylate tubes. The maximum operating pressure is $0.4 \mathrm{MPa}$ with the acrylate section and $1 \mathrm{MPa}$ with a stainless steel section.

Gas is separated from the liquid in a vessel with a diameter of $0.4 \mathrm{~m}$, see figure 5.2. At the start of the experiments the level in the separator is always $20 \mathrm{~mm}$ above the test section. Air is vented to the atmosphere or caught in a constant pressure tank; liquid is returned to the pump.

\subsubsection{Measurement of pressure drop}

Pressure is measured over a $2 \mathrm{~m}$ long section with a pressure difference transducer, Druck PDCR $120 \mathrm{WL}$, range $17.5 \mathrm{kPa}$, accuracy $0.1 \%$ of the maximum scale value. The device is calibrated with a water micromanometer (up to 0.9 $\mathrm{kPa}$ and $2.5 \mathrm{kPa}$ ) and a mercury micromanometer (from $-10 \mathrm{kPa}$ to $17.5 \mathrm{kPa}$ ). The sensitivity differs less than $0.3 \%$ for the three ranges and the combined non-linearity and hysteris is less than $0.25 \%$ for the lowest range and for the other ranges less than $0.1 \%$ of the full scale value. The diameter of the pressure holes, located in the lower side of the flanges, is $3 \mathrm{~mm}$.

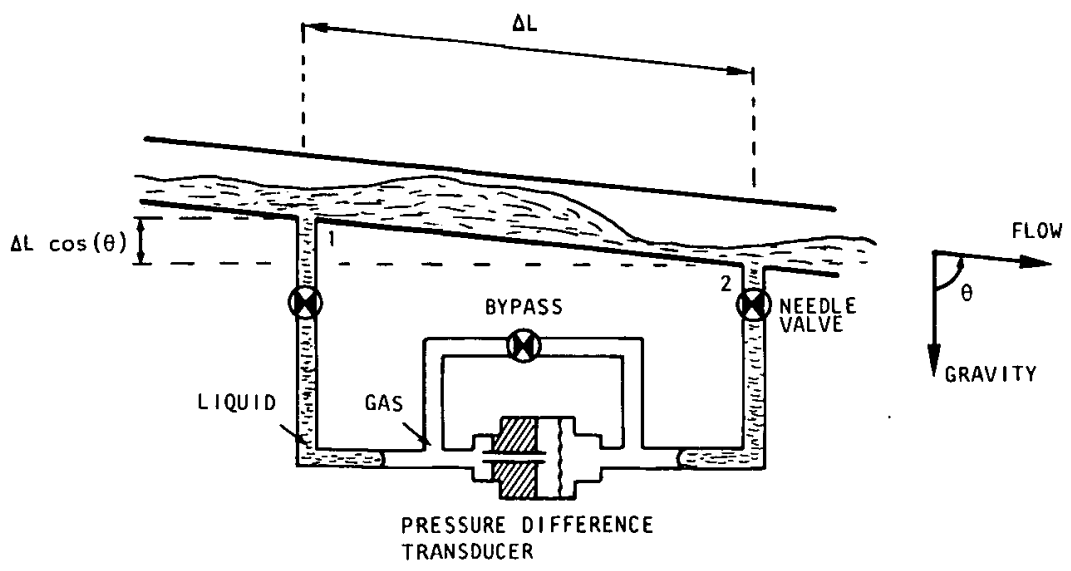

Figure 5.3: Measurement of pressure drop. 
The pressure is transmitted from the pressure holes to the transducer through partially liquid-filled lines ( $1 / 4$ inch transparent tube), see figure 5.3. At the low-pressure side of the transducer the passage from the tube connection to the space in front of the diaphragm is $1 \mathrm{~mm}$. To prevent disturbance of the pressure due to formation of liquid drops at the small opening the transducer must be either completely filled with liquid or gas. The latter solution is chosen and therefore the part of the tapping lines close to the transducer is not vented. The transparent tube is attached to a beam which is carefully placed horizontally. Hydrostatic pressure differences due to the moving gas/liquid surfaces in the tube are negligible then.

The measured averaged pressure drop appeared to be independent of the amount of gas in front of the transducer. Using needle valves, placed near the pressure taps, pressure fluctuations can be damped. Even if the fluctuations are completely suppressed, the averaged pressure drop remains the same within the level of reproducibility $( \pm 4 \%)$. During the scaling test the valves are fully open. The frictional pressure drop, $\Delta \mathrm{p}_{\mathrm{f}}$, is the difference of the measured pressure drop, $\Delta p_{m}$, and a gravitational term, due to the difference in height of the pressure taps with respect to the horizontal, see equations $4.2-4.5$ :

$$
\Delta p_{f}=\Delta p_{m}-\alpha \rho_{L} g \Delta L \cos (\theta)
$$

\subsubsection{Supply of liquid and gas}

The scheme for circulating liquid and gas is depicted in figure 5.4. It is very similar to the one used for the $5 \mathrm{~mm}$ test section (figure 4.16). The same equipment is used for circulating and conditioning the fluids, except that the vessels with the balloons are left out and to adjust high liquid flow rates $\left(U_{L}>0.5\right.$ ) the centrifugal pump $B$ is used. Gas flow rates and low liquid flow rates are measured with integral orifice plates. High liquid flow rates are calculated from the measured single-phase liquid pressure drop over $6 \mathrm{~m}$ in the return line $(D=49.8 \mathrm{~mm}$ ) to the pump $B$, using the Blasius-equation. The error is 2.5\%, malnly due to the error in adjusting the desired flow rate with the centrifugal pump $B$. Using orifice plates the tolerance in the liquid flow is $1.2 \%$ and in the gas flow $1.6 \%$, see 
table 4.2. The flow meters are calibrated very conscientiously (with water and $a(r)$ and checked frequently.

The pressure in the gas outlet, measured with a precision gauge (instrument accuracy $=1 \mathrm{kPa}$ ) is constant within $\pm 1 \%$. The temperature in the gas outlet varies by $\pm 1 \%$.

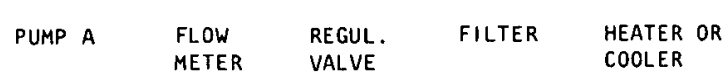

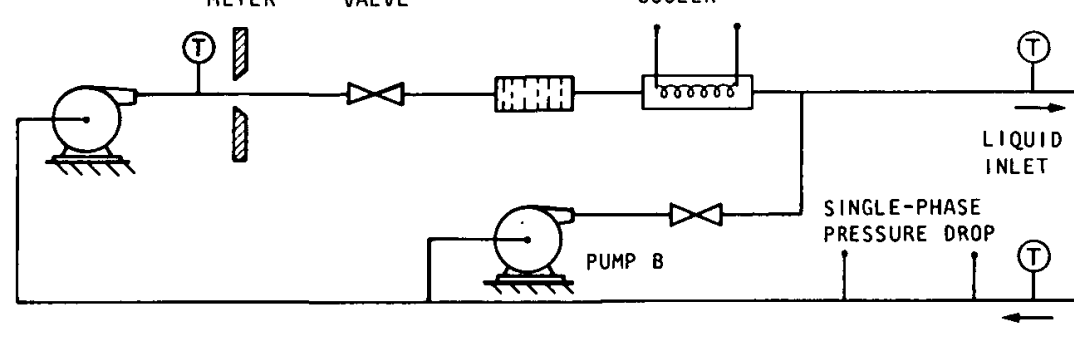

LIQUID

OUTLET

AIR SUPPLY PRESSURE

$15 \mathrm{MPa}$ CONTROL

$\begin{array}{lll}\text { FLOW } & \text { REGUL. FILTER } \\ \text { METER } & \text { VALVE }\end{array}$

ELECTRIC

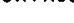

(P) (T)

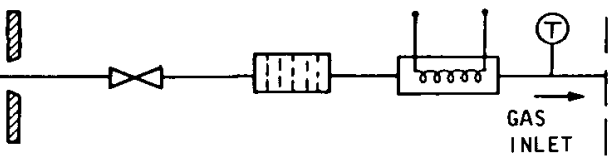

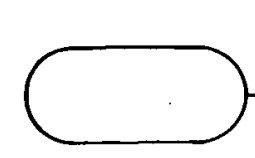

TANKS, $70 \mathrm{~m}^{3}$ FOR CONSTANT PRESSURE

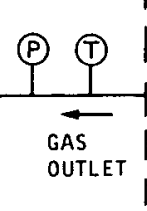

OUTLET

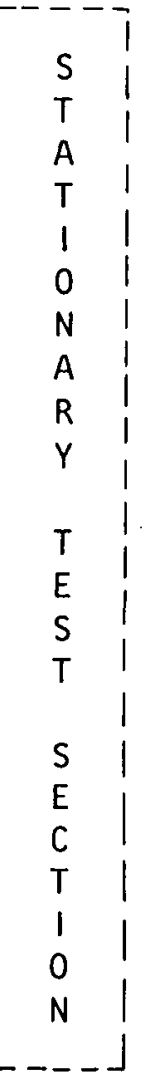

F1gure 5.4: Scheme for circulating liquid and gas. 
5.1.4 General procedure for measurement

Before a set of experiments is started, the density, specific conductivity and occasionally the viscosity of the liquid is measured. Due to vaporization the composition of the water/glycerine mixture changes with time. Due to the uncertainty of the temperature and composition of the liquid the tolerance in the density is $\pm 0.3 \%$, in the viscosity $\pm 4 \%$ and in the surface tension $1.5 \%$.

Before gas is added to the flow first the sensor output and single-phase 1 iquid pressure drop is measured. The deviation from the Blasius value (equation 4.1) is always less than $\pm 4 \%$. The two-phase pressure drop is divided by the calculated single-phase pressure drop, to obtain the friction multiplier $\phi_{L}^{2}$.

After the two-phase flow is adjusted at least three recordings of the averaged output signals (averaging time is 80 seconds) of the pressure difference transducer, the void sensor and the flow meters are carried out. From the three values it is checked whether the averaging time is sufficient. The difference between two measurements is always less than $6 \%$ for pressure drop and less than 0.01 (absolute units) for the vold fraction. For calculations the averaged value of the three measurements are used.

The void fraction is calculated with the help of the capacitance of the liquid filled sensor and gas filled sensor (see also equation 3.26):

$$
\alpha=\frac{C_{\text {mix }}-C_{11 q}}{C_{\text {gas }}-C_{11 q}}
$$

For stratifled flow patterns the calibration curve is used: see figure 3.14. The maximum absolute error in the void fraction measurement is \pm 0.03 . The long term (week/month) reproducibility of the fricion multiplier is $\pm 4 \%$ and \pm 0.01 for the vold fraction.

High demands are made upon the adjustment of a particular test condition. The scheme depicted in figure 4.17 is used for the $5 \mathrm{~mm}$ as well as the $50 \mathrm{~mm}$ test section. Different operators obtain the same results within the range of the reproducibility. A view of the tolerances of the adjusted parameters and dimensionless groups is given in table 4.2 . 
The time-varying pressure and void signals are recorded over a period of 20 seconds with a digital oscilloscope (Nicolet 3091 , sample frequency is $0.2 \mathrm{kHz}$ ).

The flow pattern and plug or slug frequency is determined from the void signal as described in section 3.5. Initially the observations from the transparent section were useful in interpreting the void signal.

\subsection{PRELIMINARY EXPERIMENTS}

To gain insight into the sensitivity of the experimental results to secondary parameters or loop dependent parameters such as the filling degree of the separator, the inclination angle or the position of the pressure taps, these parameters were individually varied.

\subsubsection{Influence of the separator}

The influence of the filling degree of the separator was investigated with a water/glycerine mixture at atmospheric pressure and downward inclination $1: 113$. Since no void sensor was available at that time only the total pressure drop and flow pattern could be compared. Usually the liquid level in the separator is $20 \mathrm{~mm}$ above the entrance of the test section in the separator (the tube being completely filled with liquid).

A $100 \mathrm{~mm}$ rise of the level has no inluence on the measured total pressure drop.

If the level is lowered in such a way that the separator level is always (the level rises due to the presence of gas in the test section) below the liquid surface, then only in one case $\left(a_{24}\right)$ was a significant $(6 \%)$ lower total pressure drop measured. The flow pattern was not influenced.

With the separator level above the test section back flow of a liquid layer of thickness $5-10 \mathrm{~mm}$ is observed at the lowest flow rates (test condition $a_{11}$ ). The resulting front stabilized somewhere between the pressure taps and the separator. Sometimes it was perceptible in the transparent section. At smaller downward inclination angles backflow was also observed at the test condition $a_{12}$, affecting the measurement of vold fraction and pressure drop. 


\subsubsection{Influence of the inclination angle}

To fulfil the requirement of geometrical similarity most experiments are performed at small downward inclinations (often 1:113). By varying the effective gravitational acceleration in the rotating test section not only the Froude number is changed but also the inclination angle. To distinguish between the true Froude effect and the slope-effect, the influence of the inclination of the tube on the pressure drop and flow pattern was studied. It is well known in two-phase flow that the flow pattern is very sensitive to the slope of the tube, especially in upward flow (Barnea et al. 1980, Spedding and Chen 1982, Taitel and Dukler 1976).

A comparison of the friction multiplier for a horizontal test section and a tube with downward inclination 1:113 is shown in figure 5.5. For the test series $A_{3}$ vold fraction was not measured and the values of series $A_{1}$ were therefore used to calculate the frictional part of the pressure drop.

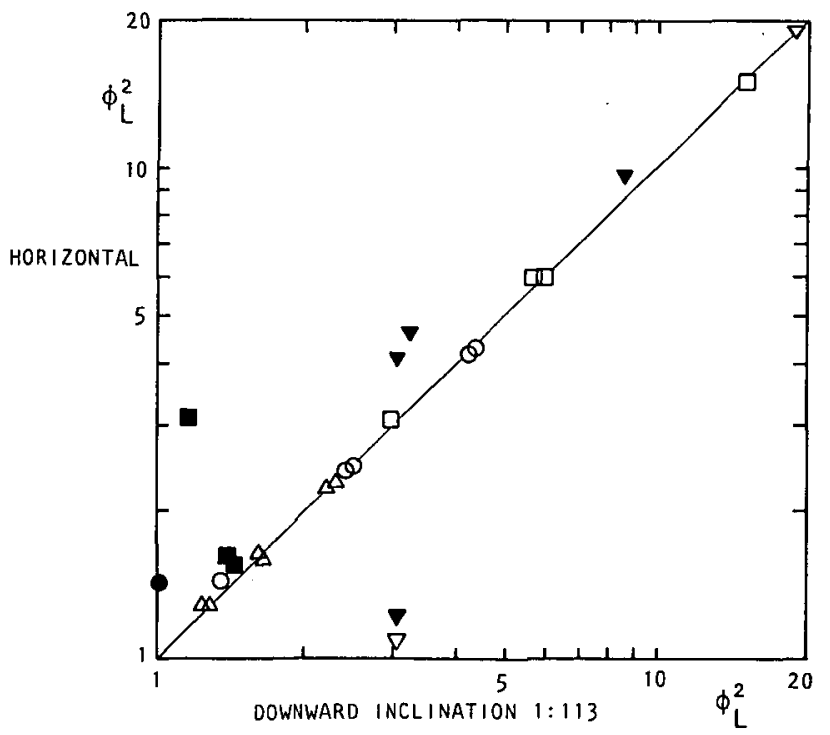

Figure 5.5: comparison of the friction multiplier for horizontal flow (test series $A_{2}$ and $A_{4}$ ) and inclined flow (series $A_{1}$ and $A_{3}$ ), closed symbols: different flow pattern, standard symbols used. 
At low fluid velocities ( $\nabla$ and $\square$ ) the flow pattern and hence the pressure drop are very sensitive to the inclination angle. The closed symbols represent a transition from stratified flow at downward inclination 1:113 to plug flow in the horizontal case. The measurements of the test condition $a_{11}$, at which backflow of the separator occurs (see section 5.2.1), deviate from the general trend. At high liquid velocities the differences are small and only for the test condition $a_{3 i}$ is the flow pattern changed.

\subsubsection{Flow development}

The term "fully developed flow" at a certain position along the tube is used here if the flow downstream of that particular position is identical. In reality a two-phase flow is never fully developed because of expansion of the gas. However if the pressure drop is low and the operating pressure small, this effect is slight. The flow may be characterized by a designation of the flow pattern, the pressure drop, the void fraction, etc.

Weisman et al (1979) have found from visual observations in a $51 \mathrm{~mm}$ diameter tube that the differences in the flow pattern between $60 \mathrm{D}$ and $120 \mathrm{D}$ are very small. They also noted that the inlet geometry has no significant influence on the final flow pattern.

To study the flow development, pressure drop was measured consecutively over the usual $2 \mathrm{~m}$ long section and over a $2 \mathrm{~m}$ long section upstream. The same pressure difference transducer was used and the connections with the desired pressure taps being made with the help of valves. The measurements were carried out with the water/glycerine mixture at $0.44 \mathrm{MPa}$ and downward inclination 1:113. Significant differences were observed: at intermittent flows the total pressure drop over the upstream section is on the average $4 \%$ lower and at stratified flow even $20 \%$. Large differences are measured for the test conditions $a_{11}:-30 \%$ and $a_{21}$ : - 40\%. The same results were obtained if the two pressure drop sections are exchanged. Because the measurements are carried out at a small inclination of the test section, the differences can be caused by a developing frictional pressure drop as well as a developing void fraction. The contribution of the gravitational part of the measured pressure drop (see equation 5.1) to the measured pressure drop itself is considerable at stratified flow pattern: see table 5.1 . 


\begin{tabular}{|lll|lll|lll|lll|}
\hline $\begin{array}{l}\text { test } \\
\text { cond }\end{array}$ & FP & Ratio & $\begin{array}{l}\text { test } \\
\text { cond }\end{array}$ & FP & Ratio & $\begin{array}{l}\text { test } \\
\text { cond }\end{array}$ & FP & Ratio & $\begin{array}{l}\text { test } \\
\text { cond }\end{array}$ & FP & Ratio \\
\hline$a_{11}$ & SS & 0.57 & $a_{21}$ & SS & 0.25 & $a_{31}$ & SS & 0.06 & $a_{41}$ & PL & 0.01 \\
$a_{12}$ & SS & 0.55 & $a_{22}$ & PL & 0.22 & $a_{32}$ & PL & 0.14 & $a_{42}$ & PL & 0.02 \\
$a_{14}$ & SW & 0.15 & $a_{24}$ & SL & 0.15 & $a_{33}$ & PS & 0.12 & $a_{43}$ & PL & 0.02 \\
$a_{15}$ & SA & 0.12 & $a_{25}$ & SA & 0.06 & $a_{34}$ & PL & 0.11 & $a_{44}$ & SL & 0.02 \\
\hline
\end{tabular}

Table 5.1: Ratio of gravitational part of the measured pressure drop to the measured pressure drop itself for the test conditions of the scaling test $A$.

Another reason for the large deviations at small flows could be back flow of liquid from the separator, as reported in the previous section. If the back flowing front stops somewhere in the upstream pressure section then the height of the liquid layer in the tube is different at the three pressure taps. A difference of $5 \mathrm{~mm}$ between the middle and upstream pressure tap would be sufficient to cause the observed deviations.

\subsubsection{Conclusions}

Accurate and reproducible measurements of the pressure drop, void fraction and flow pattern can be carried out with the $50 \mathrm{~mm}$ test facility.

The liquid level in the separator has no influence on the flow pattern and pressure drop, except for very low liquid and gas velocities where back flow occurs.

Small downward inclination angles are of only influence on the frictional pressure drop if the flow pattern is affected and this only happens at low flow velocities. The influence of the changing inclination angle on the flow at the test series $A_{13}$ and $A_{14}$ (variation of the Froude number via variation of the rotation rate) can therefore be neglected.

The total pressure drop, especially for stratified flows, is dependent on the distance from the mixing section. Back flow of liquid from the separator at low fluid velocities is partially responsible for this. 


\subsection{THE 100 MM TEST FACILITY}

The experiments with the $100 \mathrm{~mm}$ test loop were carried out at the Koninklijke/Shell Laboratorium in Amsterdam (KSLA).

In this section a description of the test facility, pressure drop measurements and void fraction measurements is given, together with some results on the influence of the entrance device on the two-phase flow. A review of the test conditions, fluid properties and experimental results can be found in Appendix II.

\subsubsection{Description of the loop}

The horizontal test section consists of a steel pipe with an inner diameter of $105 \mathrm{~mm}$. The total straight length is $32 \mathrm{~m}$. Three entrance devices for the gas were tested (see figure 5.6):

geometry 1: gas inlet from above, at $26^{\circ}$ to the horizontal

geometry 2: gas inlet from below, at $26^{\circ}$ to the horizontal

geometry 3: gas inlet $14 \mathrm{~m}$ upstream of the $180^{\circ}$ bend. The diameter of the gas inlet line was $40 \mathrm{~mm}$. Configuration 1 is geometrical similar to the mixing device of the 5 and $50 \mathrm{~mm}$ test section and the results obtained with this configuration are therefore used for comparing the flows.

Pressure drop was measured over $15.1 \mathrm{~m}$ (143 diameters) after a straight length of $9.8 \mathrm{~m}$ ( 93 diameters). The void meter was located $16 \mathrm{~m}$ (150 diameters) from the mixing section. A transparent section, $1 \mathrm{~m}$ long, is used for visual observations.

Gasoil is circulated through the test section by a centrifugal pump. The flow rate is adjusted with the help of regulating valves and a bypass over the pump. Small flow rates $\left(0.2<U_{L}<1.6\right)$ are measured with uncalibrated orifice plates and high flow rates $\left(0.6<U_{L}<1.6\right)$ with a turbine flow meter. The error in the velocity measurement is estimated at $\pm 3 \%$ : see table 4.2 . 


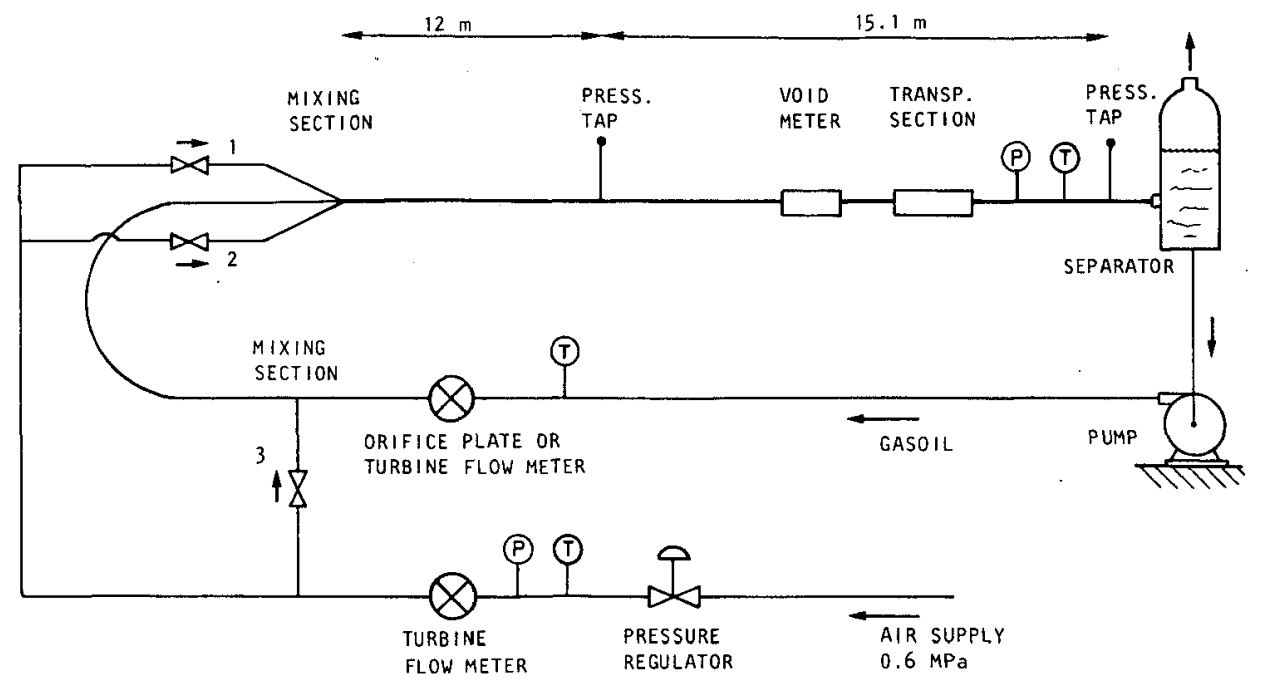

Figure 5.6: The $100 \mathrm{~mm} 100 \mathrm{p}$.

Compressed air is used from a $0.6 \mathrm{MPa}$ supply. The gas flow rate is measured with turbine flow meters. The actual values of the temperature and pressure upstream of the flow meters and at midpoint of the test section are used for calculating the superficial gas velocity. The total error in the velocity measurement is estimated to be $3 \%$. For the properties of the air the data for dry air are taken. At the end of the straight length gas is separated from the liquid and vented to the atmosphere.

The absolute pressure at the midpoint of the test section is calculated from the measured value of the absolute pressure at the downstream pressure tap and the two-phase pressure drop. For the total range of conditions the averaged absolute pressure is $0.11 \mathrm{MPa}$. At low velocities it is $5 \%$ lower, at high velocities $5 \%$ higher.

The temperature of the liquid increases up to $10^{\circ} \mathrm{C}$ during the day because of heat input of the sun and pump. The averaged temperature of the tests, concerning the scaling test $\mathrm{B}$ is $37^{\circ} \mathrm{C}$ and the maximum variation is $\pm 3^{\circ} \mathrm{C}$.

The density, viscosity and surface tension are measured. Due to the variation in the temperature the uncertainty in the density is $\pm 0.4 \%$ and in the viscosity $\pm 7 \%$. The tolerance in the measurement of the surface 
tension is $\pm 5 \%$. For calculations the averaged values of the whole test series are used. A survey of the errors in the main parameters and dimensionless groups is given in table 4.2 .

\subsubsection{Measurement of pressure drop}

The pressure is transmitted from the taps in the bottom of the pipe to the pressure difference transmitters through liquid filled lines. Care is taken to exclude air from the tapping lines. Four transmitters (span: 1.25 $\mathrm{kPa}, 5 \mathrm{kPa}, 25 \mathrm{kPa}$ and $200 \mathrm{kPa}$ ) are connected simultaneously to the pressure taps. The computer code chooses the transmitter with the lowest range provided none of the 40 momentary values (see section 5.3.4), from which the averaged values are calculated, exceeds the full scale value. Because the averaged pressure drop is much smaller than the momentary one, the value always lies in the lower part $(0-40 \%)$ of the span. In half of the adjusted flows even less than $20 \%$ of the span is in use. The error of the pressure drop measurement is therefore large, typically $\pm 5 \%$. In single-phase flow the whole span is effective and the accuracy is better.

The friction multiplier is calculated from the measured two-phase pressure drop and the calculated single-phase liquid pressure drop, using the Blasius equation.

\subsubsection{Measurement of vold fraction}

The vold content is measured with the so-called neutron back-scattering method (NBS), based on the interaction of neutrons from a californium 252 source with hydrogen atoms. Upon interactions with hydrogen atoms the neutrons loose the largest part of their energy. The number of these low energy neutrons is a measure of the amount of liquid in a certain volume, provided the liquid contains hydrogen atoms. Interactions of neutrons with heavier materials are negligible. The scattered neutrons are counted by a Helium-3 detector. Three source-detector units are placed symmetrically around the tube. A pipe length of at least $0.2 \mathrm{~m}$ is observed by the NBS gauges.

The sum-signal of the three detectors is amplified and fed to a rate-meter. The integration time of the rate-meter is adjusted to a small value $(0.5 \mathrm{~s})$ in order to detect vold fluctuations as good as possible. 
Figure 5.9 shows some recorded signals. Since the relationship between the void fraction and the signal of the rate-meter is not linear only the two extremes full (liquid) and empty (gas) are indicated. If the signal reaches the level full then the sensitive volume has been completely filled with liquid during at least $0.5 \mathrm{~s}$. The device is calibrated by filling a horizontal section with water, the void meter being fixed at its mid-point, see figure 5.7 .

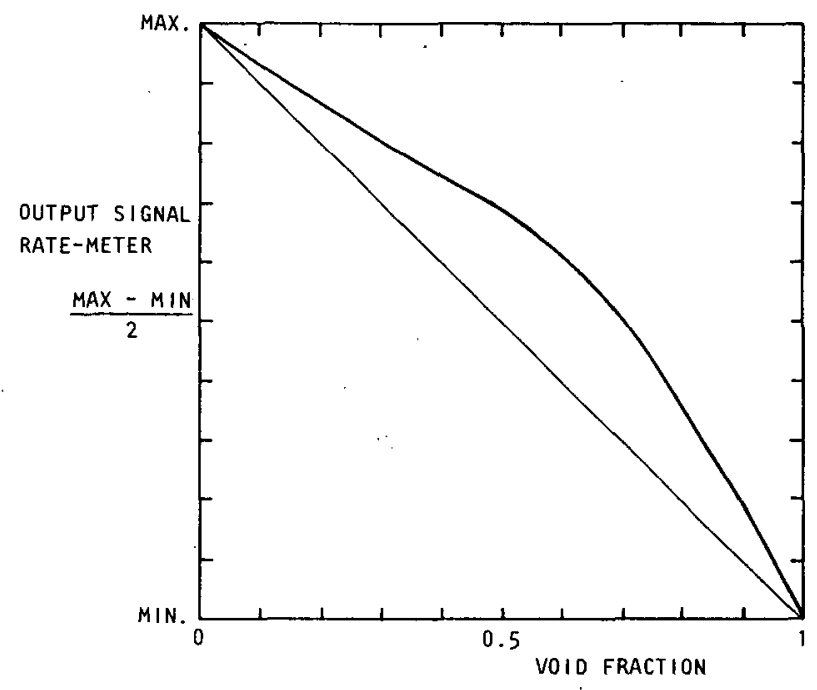

Figure 5.7: Calibration curve for stratified flow.

The void fraction is calculated from the height of the liquid level. It is assumed that the form of the calibration curves for water and gasoil are equal. The calibration curve obtained in this way is only valid for stratifled flow. For intermittent flow the measured void fraction will be too high in general. Consider for example an idealized plug flow as indicated in figure 5.8. Locally the flow is stratified and the calibration curve of figure 5.7 can be applied. The measured sum-signal equals $1 / 2$ $[(\max -\min )+0.38(\max -\min )]=0.69(\max -\min )$ which corresponds to a void fraction of 0.5 , whereas the actual value is $(0.8+0) / 2=0.4$. The measured void fraction is thus strongly dependent on the flow pattern. 


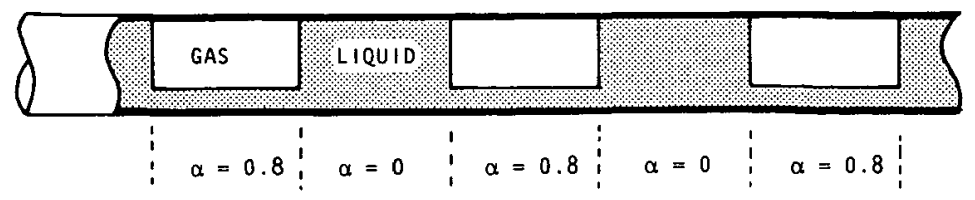

Figure 5.8: Idealized plug flow.

If the local vold fraction is always smaller than 0.6 the error due to averaging of the sum signal is small. This situation occurs at $U_{L}>1$ with $\mathrm{U}_{\mathrm{G}}<3$ and $\mathrm{U}_{\mathrm{L}}<0.5$ with $\mathrm{U}_{\mathrm{G}}<1$. Beyond this range, deviations of up to +0.1 are to be expected at high gas velocities $\left(U_{G}>3\right)$. The deviations are in the first place due to the integrating nature of the NBS method in connection with the non-linear calibration curve. Moreover the "instantaneous" (smallest integration time $0.5 \mathrm{~s}$ ) output signals of the rate-meter are averaged rather than the "instantaneous" vold fraction, see section 5.3 .4 .

\subsubsection{Data aquisition and reproducibility}

With the help of a data scanner the output signals (18 in all) of the flow meters, differential pressure transmitters, void meters, pressure and temperature gauges are transmitted to a Hewlett Packard desk computer (model 9825A). For processing one instantaneous value of all signals, called a "scan", about nine seconds is needed. The averaged values of 40 scans are used for calculating the superficial fluid velocities $U_{L}$ and $U_{G}$, the pressure drop, the vold fraction (with the help of the calibration curve), the temperature and the absolute pressure at the midpoint of the test section. The results are printed. The time-varying void signal from the rate-meter is registrated continuously with an $x-t$ recorder.

To check whether 40 scans is sufficient to obtain reasonable averaged values two successive measurements are carried out. The void fraction differs always by less than 0.02 , whereas the pressure drop differs by less than $6 \%$ in 12 out of 15 examined conditions, covering the whole experimental region. Occasionally large differences ( $30 \%$ and $80 \%$ ) are observed.

A systematic check of thellong term reproducibility is not carried out because it takes a great deal of effort to adjust a specific liquid and gas 
velocity (a direct feed back is not available). An estimation, based on a few comparible measurements, indicates that the long term reproducibility is not better than $\pm 10 \%$ for the pressure drop and \pm 0.03 for the vold fraction.

The relative large deviation of the single-phase liquid pressure drop from the Blasius equation also indicates that the long term reproducibility is not better than $\pm 10 \%$. For the range $6000<$ Re $<60000$ the standard deviation for the relative difference between the measured values and the values from the Blasius equation is $6.5 \%$. No systematic differences which for instance might be caused by wall roughness are observed.

\subsubsection{Experimental: influence of gas inlet geometry}

Weisman et al. (1979) and Hoogendoorn (1959) have reported that the influence of the inlet geometry is negligible beyond $60 \mathrm{D}$. Simpson et al. (1977) on the contrary found differences between experiments with different inlet geometries even after $128 \mathrm{D}$. In vertical bubble flow a strong influence of the inlet geometry and liquid purity on the vold fraction is reported by wallis (1969).

Here three different entrance devices for the air are tested, see figure 5.6. The time-varying void fraction is measured after 150 diameters. To assure that the same flow conditions are used, the three measurements are carried out in close succession. The velocities differ by less than $\pm 4 \%$. From the fifteen examined test conditions, the difference between the largest and smallest pressure drop is $15 \%-30 \%$ in four cases with high gas velocities $\left(U_{G}>3 \mathrm{~m} / \mathrm{s}\right)$. In seven cases, which include the four mentioned above, the void fraction differs by more than 0.06 (see for example figure $5.9 \mathrm{~d} \alpha_{1}=0.75$ and $\left.\alpha_{2}=0.61\right)$. At low gas velocities $\left(U_{G}<1 \mathrm{~m} / \mathrm{s}\right)$ the pressure drop differs by less than $10 \%$ and the vold fraction by less than 0.05 .

From the time-varying void signal the influence of the entrance device on the flow pattern is studied. In ten cases differences are observed and six of them, not necessarily the one described above, are very distinctive, see figure 5.9. At low liquid velocities $\left(U_{L}<0.4 \mathrm{~m} / \mathrm{s}\right)$ and $10 \mathrm{w}$ gas velocities $\left(U_{G}<2 \mathrm{~m} / \mathrm{s}\right.$ ) the slug frequency with the entrance device 3 (gas inlet before the bend) is a factor of 3 larger than that for the other geometries (figure 5.9a). Some of the differences may be due to vibrations of the test section at high flow rates. 


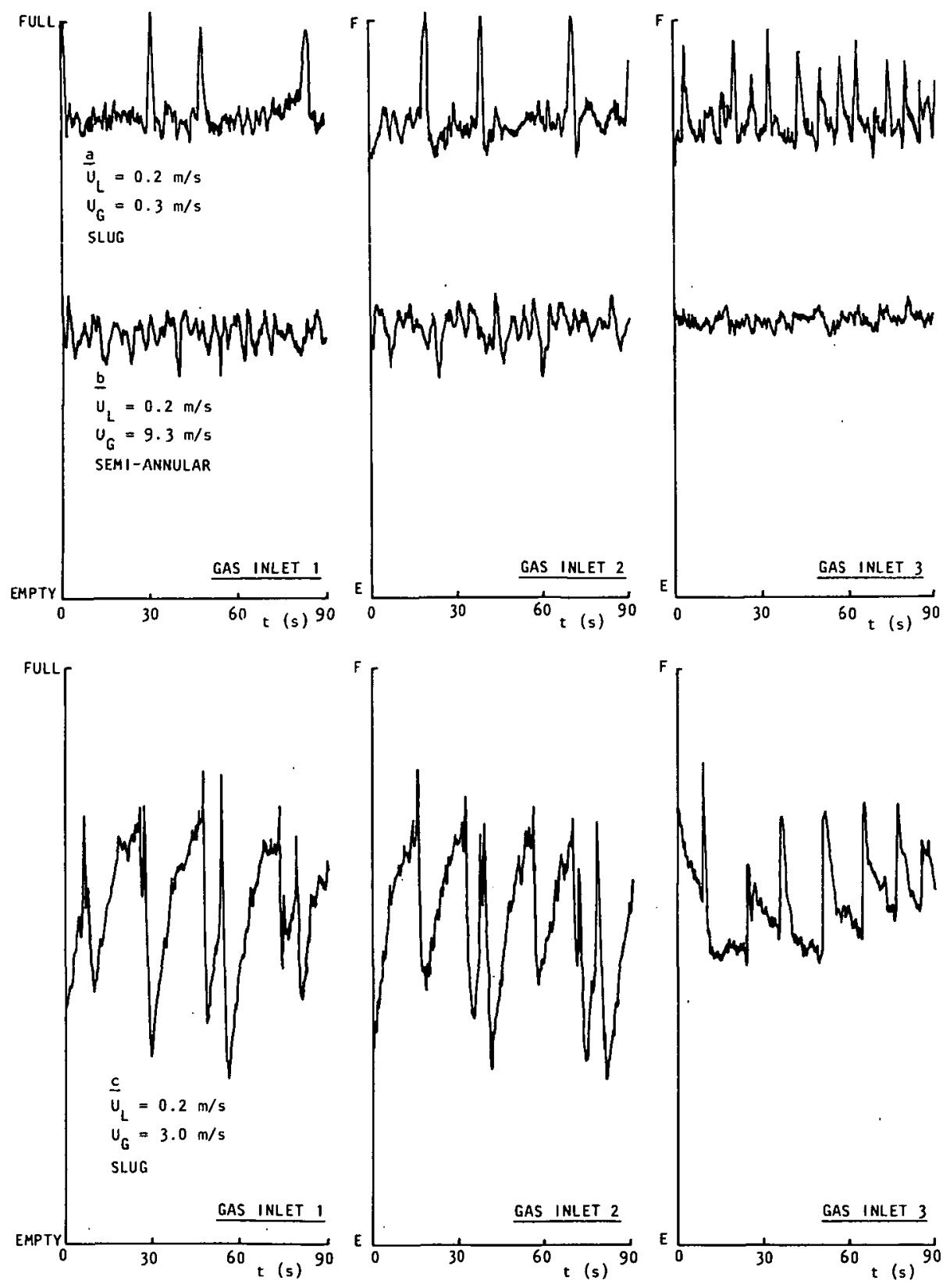

Figure 5.9a,b,c: Influence of the gas inlet geometry on the vold signal. 

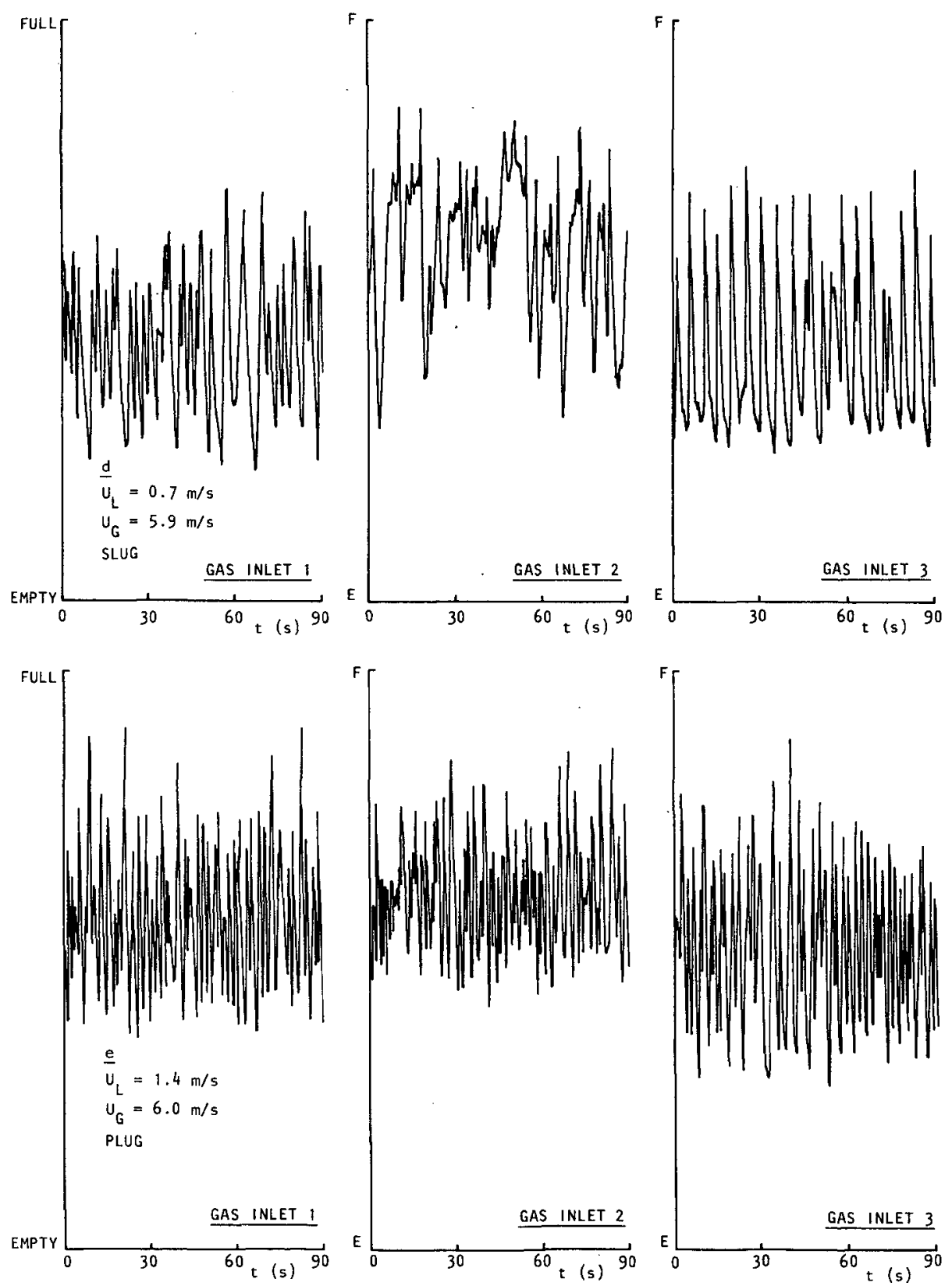

Figure 5.9d,e: Influence of the gas inlet geometry on the void signal. 


\subsubsection{Conclusions}

The accuracy of the $100 \mathrm{~mm}$ test facility is clearly lower than that used for the 5 and $50 \mathrm{~mm}$ tubes. The major reasons are:

- The liquid is not pure: anti-static additives are used, causing a foamy flow, especially at high gas velocities.

- Due to the integrating nature of the NBS method the accuracy of the vold fraction measurement is poor. At high gas velocities $\left(U_{C}>1 \mathrm{~m} / \mathrm{s}\right)$ the measured value is too high by up to +0.1 . The reproducibility is \pm 0.03 . - Half of all measured pressure drops cover less than $20 \%$ of the transducer range. Hence the accuracy of the measurement is $\pm 5 \%$ and the reproducibility of the friction multiplier not better than $\pm 10 \%$ then.

- The error in the velocity measurements are relative large since no regular checks and calibrations are carried out.

From the time-varying void signal it is evident, despite of the relatively large errors indicated above, that the geometry of the mixing section has a significant influence on the flow pattern, especially at high gas velocities. In these cases large differences in the pressure drop and void fraction are observed. At low gas velocities the plug/slug frequency with the mixing section located before the bend is much higher (by a factor of 3 ) than with the mixing section after the bend. 


\subsection{LENGTH SCALE FACTOR OF 0.1 : THE SCALING TEST A}

The measurements of pressure drop, void fraction and flow pattern in the tests of the $50 \mathrm{~mm}$ section $\left(A_{1}\right)$ are compared with those of the rotating $5 \mathrm{~mm}$ tube (test series $A_{7}$ ). Eighteen test conditions are examined, composed of four liquid velocities (indicated by the symbols $\nabla, \square, \circ$ and $\Delta$ ) and $f$ ive gas velocities, Altogether 181 data are used.

The Reynolds number, Froude number, Weber number and gas-liquid density ratio are equal in both systems whereas $(E u)_{7} /(E u)_{1}=0.66$ and $\left(\mu_{G} / \mu_{L}\right)_{7} /\left(\mu_{G} / \mu_{L}\right)_{1}=3.57$. Actually the measurements of the scaling test $B$ concerning the 5 and $50 \mathrm{~mm}$ test sections could have been used. However due to the high operating temperature $\left(55^{\circ} \mathrm{C}\right)$ the accuracy of these measurements is worse. Moreover the Weber number is not equal for the two tests.

\section{1 .1 Comparison of the pressure drop}

As can be seen from figure 6.1 the agreement of the frictional pressure drop is very good. Only at two test conditions with low fluid velocities, $a_{11}(\boldsymbol{V})$ and $a_{21}(\boldsymbol{a})$ is the difference more than $20 \%$. The closed symbols indicate a stratified flow pattern. If stratified flow situations are not considered the largest deviations are $+5 \%$ and $-19 \%$. It is striking that the values of $\phi_{L}^{2}$ obtained, using the second liquid velocity ( $a$ ) deviate more than 12\% from the diagonal whereas those using the third velocity ( 0 ), which is but $46 \%$ larger, deviate less than 5\%. The time-varying pressure drop signals for the test conditions $a_{21}, a_{22}$ and $a_{24}$ have already been given in figure 4.14 .

The large differences at low fluid velocities with stratified flow pattern are due to the specific construction detalls of the system, such as the entrance condition, the exit condition, the exact position of the tube, vibrations of the system etc. The deviations are not caused by the limited accuracy of the measurements because the experiments can always be reproduced very well, pressure drop within $\pm 5 \%$ and void fraction within \pm 0.02 (absolute units). In section 4.3 it is shown that the gravitational 
term $\alpha \rho_{L} g \Delta L \cos (\theta)$ which must be substracted from the measured pressure drop to obtain the frictional part is large at low fluid velocities. The accompaning error in the frictional pressure drop due to the tolerance of the vold fraction $( \pm 0.03)$ and exact position of the test section and pressure taps (error $\pm 0.1 \mathrm{~mm}$ ) with respect to the rotation axis, can therefore be large too: $\pm 90 \%$ at the test condition $a_{11}, \pm 15 \%$ at $a_{12}$ and $\pm 10 \%$ at $a_{21}$. As pointed out in section 5.2.1 back flow of liquid from the separator influences the measurements at low fluid velocities with a stratifled flow pattern. In the rotating test section similar effects probably occur. The dependency of the measured pressure drop on the distance from the inlet at low fluid velocities (see 5.2.3) indicates that elther the flow is not developed yet or backflow is important.

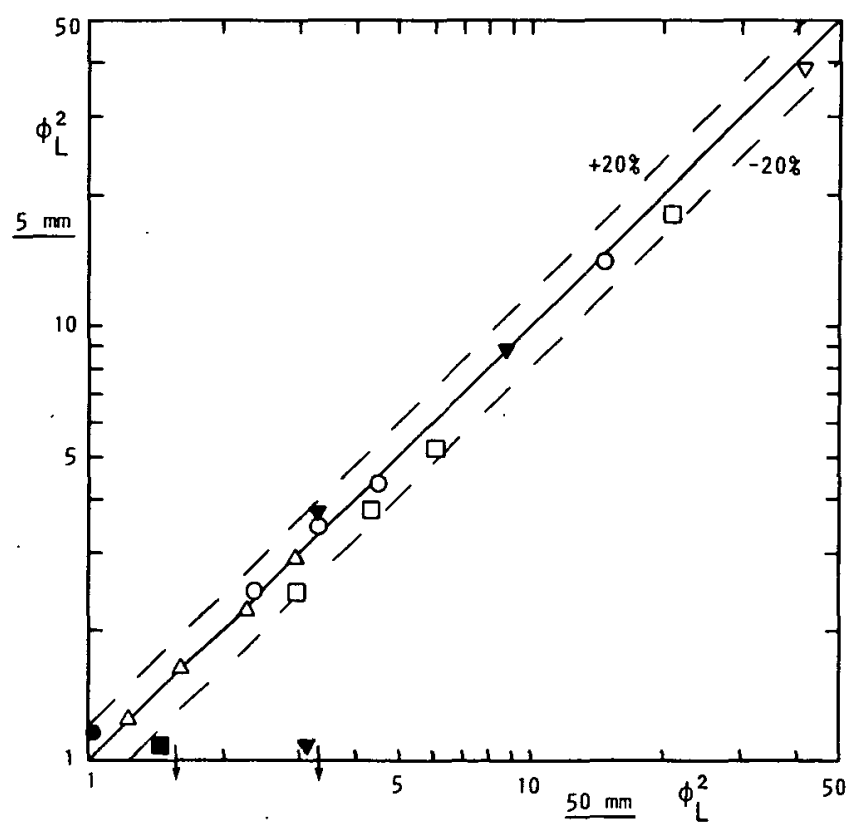

Figure 6.1: Comparison of the friction multiplier, test series $A_{1}$ and $A_{7}$, closed symbols: stratified flow. 


\section{1 .2 Comparison of the void fraction}

If stratified flow patterns (indicated by the closed symbols) are not considered then again the agreement between the $5 \mathrm{~mm}$ and $50 \mathrm{~mm}$ tests is excellent, see figure 6.2. The differences are less than 0.03 .

The deviations for stratified flow may be caused by influences of the separator and/or differences in flow development as pointed out in the previous sections.

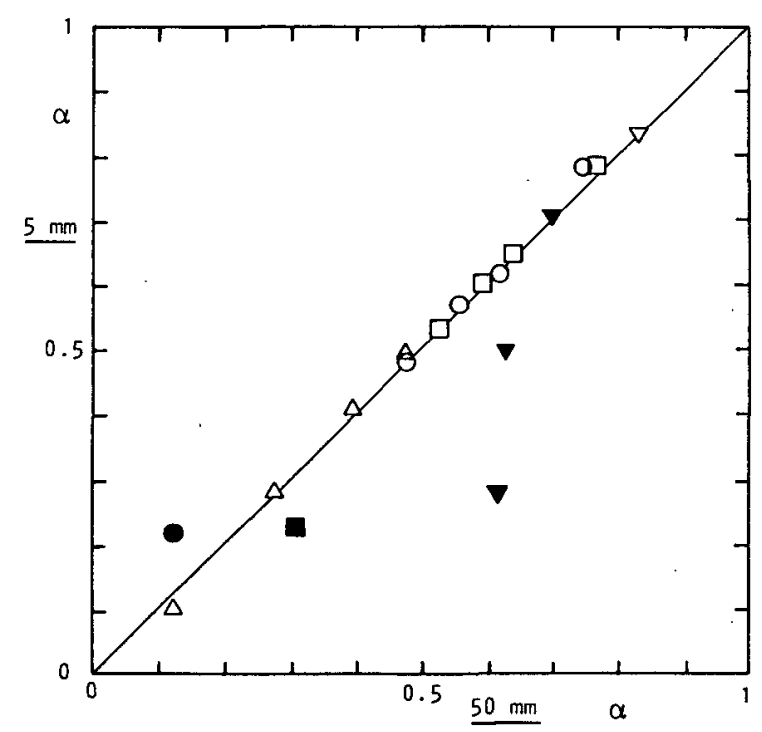

Figure 6.2: Comparison of the void fraction, test series $A_{1}$ and $A_{7}$, closed symbols: stratified flow.

\section{1 .3 Comparison of the flow pattern}

The flow pattern is deduced from the time-varying vold signals in the manner described in section 3.5. Figure 6.3a-d show the signals of the 50 and $5 \mathrm{~mm}$ sensors. Both the qualitative (flow pattern) and the quantitative (absolute values of the void fraction) agreement is very striking. Many 
pictures are similar in detail, for example the shape of the plugs ( $a_{22}$ and $\left.a_{32}\right)$ or the alternation of small and large plugs or slugs $\left(a_{32}\right.$ and $\left.a_{33}\right)$. The flow pattern for the test condition $a_{31}(\bullet)$ is stratified in the $50 \mathrm{~mm}$ section and plug in the $5 \mathrm{~mm}$ section. If the liquid velocity is raised by $10 \%$ then plug flow is also obtained in the $50 \mathrm{~mm}$ section. Possibly the transition in the rotating test section could be triggered by vibrations of the systein.

The void signals in the runs $a_{14}, a_{15}, a_{24}, a_{25}$ and $a_{35}$ using the $5 \mathrm{~mm}$ sensor are flattened because of the limited response time ( 2 msec.) of the capacitance meter, see section 3.5 .

The plug and slug frequencies are determined simply by counting the peaks in the void signals. As shown in section 3.5 this is justified if the frequencies are less than $1.3\left(U_{L}+U_{G}\right) / 6 D$, which is the case here. At least three recordings are used. The reproducibility is $\pm 10 \%$. Countings carried out by different persons produces the same result within 5\%. The observations in the transparent $50 \mathrm{~mm}$ section differ less than $10 \%$ from the countings of the void peaks except for condition $a_{32}$ where the observations of the transparent section yield a $15 \%$ higher value. In table 6.1 the plug and slug frequency for the $5 \mathrm{~mm}$ and $50 \mathrm{~mm}$ test section and the ratio $\mathrm{f}_{5} / \mathrm{f}_{50}$ are listed. The averaged frequency ratio is 34.5 and the standard deviation is 3.2. The theoretical value of the frequency scale factor is $(U / L)_{5} /(U / L)_{50}=33.8(!)$, see section 2.4 .1 .

\begin{tabular}{|l|llllllllll|}
\hline & $a_{22}$ & $a_{23}$ & $a_{24}$ & $a_{32}$ & $a_{33}$ & $a_{34}$ & $a_{41}$ & $a_{42}$ & $a_{43}$ & $a_{44}$ \\
\hline$f_{5}$ & 11.5 & 14 & 24 & 21 & 19 & 29 & 92 & 93 & 61 & 60 \\
$f_{50}$ & 0.35 & 0.45 & 0.68 & 0.52 & 0.50 & 0.82 & 2.95 & 2.57 & 1.93 & 1.82 \\
$\frac{f_{5}}{f_{50}}$ & 32.8 & 31.1 & 35.1 & 40.6 & 38.0 & 35.5 & 31.2 & 36.2 & 31.6 & 33.0 \\
\hline
\end{tabular}

Table 6.1: Plug and slug frequencies $\left(\mathrm{s}^{-1}\right)$. 
$50 \mathrm{~mm}$ TEST SECTION
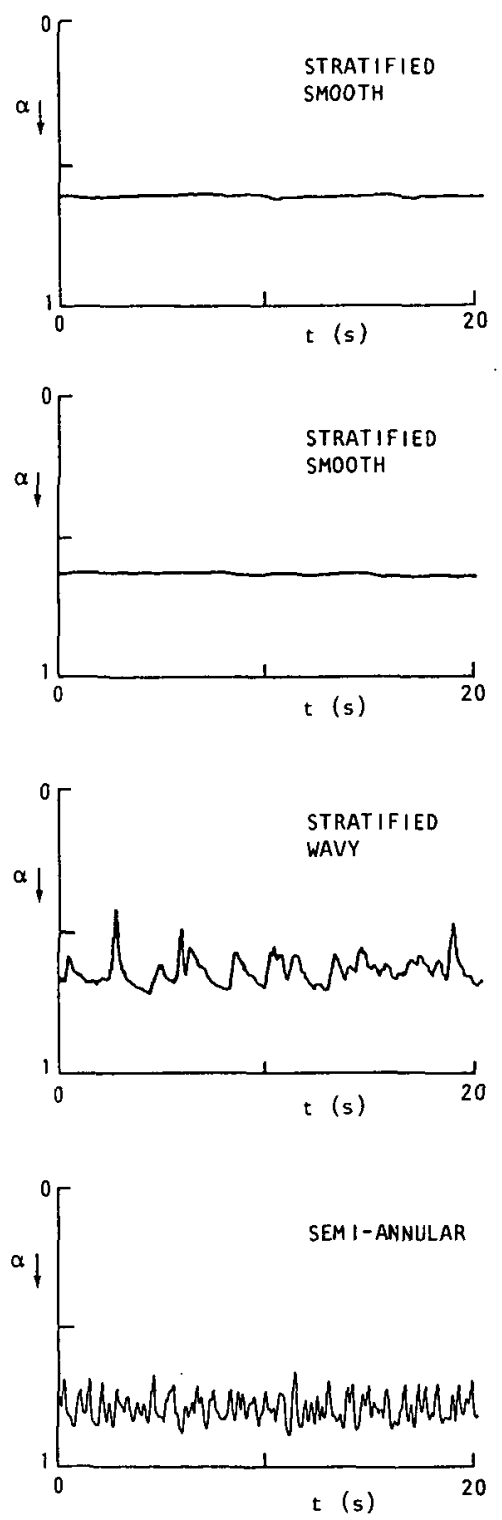

$5 \mathrm{~mm}$ TEST SECTION

STRATIFIED

SMOOTH

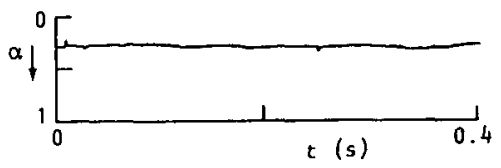

STRATIFIED SMOOTH

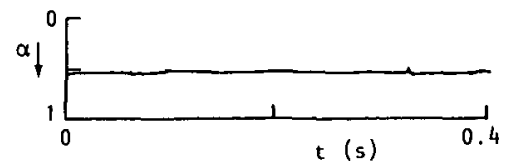
WAVY

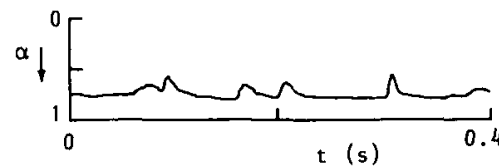

SEMI-ANNULAR

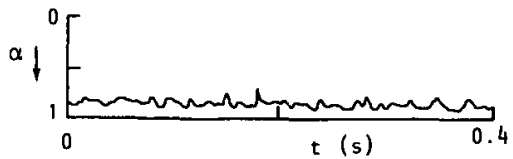

F1gure 6.3a: Time-varying vold signals of the 50 and $5 \mathrm{~mm}$ tests, test conditions $a_{11}$ (top), $a_{12}, a_{14}$ and $a_{15}$. 

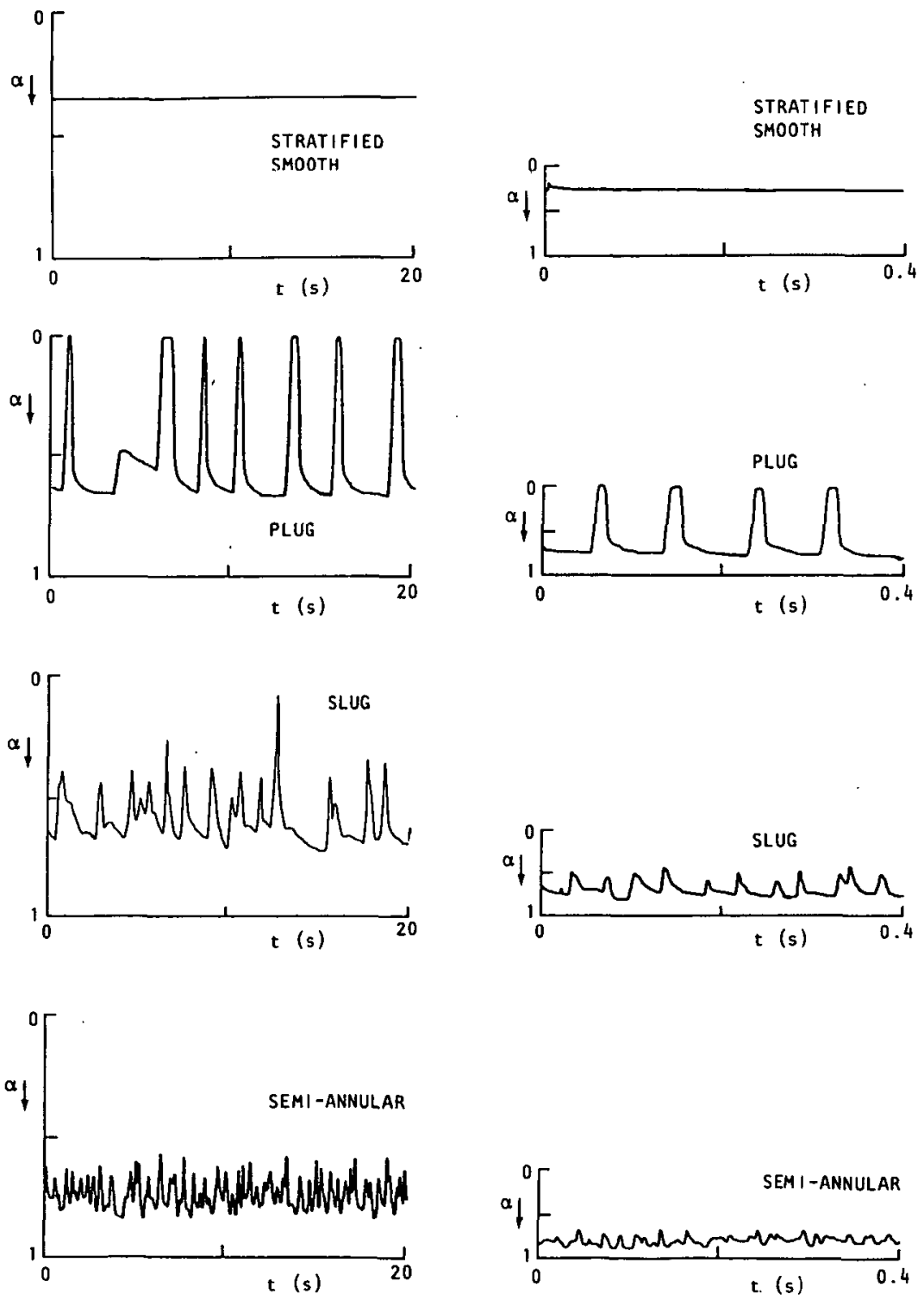

Figure 6.3b: Time-varying vold signals of the 50 and $5 \mathrm{~mm}$ tests, test conditions $a_{21}$ (top), $a_{22}, a_{24}$ and $a_{25}$. 

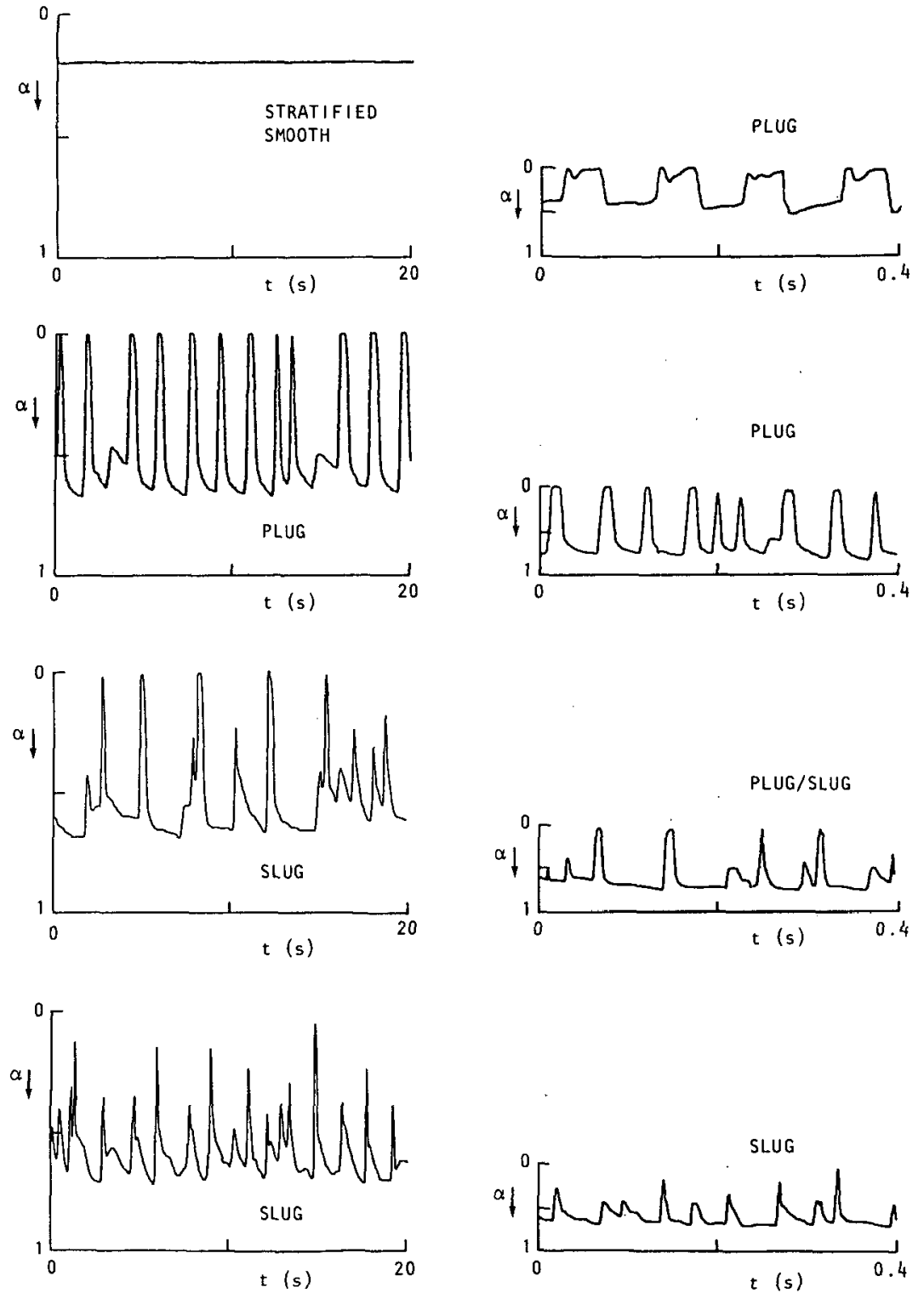

Figure 6.3c: Time-varying void signals of the 50 and 5 mrm tests, test conditions $a_{31}$ (top), $a_{32}, a_{33}$ and $a_{34}$. 
$50 \mathrm{~mm}$ TEST SECTION
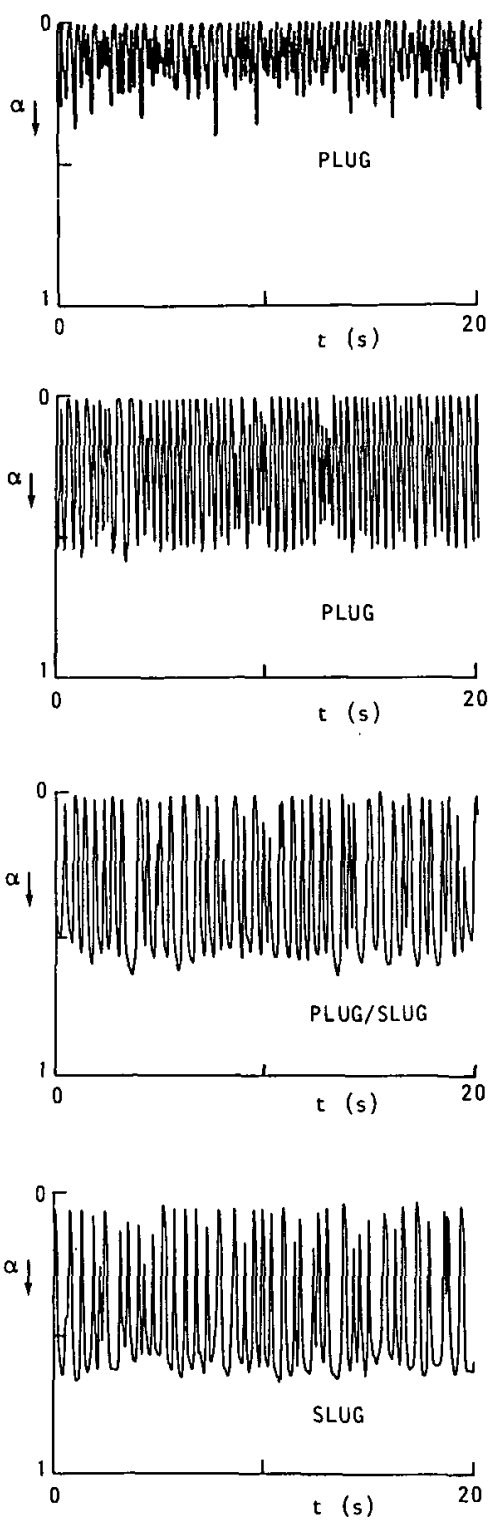

$5 \mathrm{~mm}$ TEST SECTION

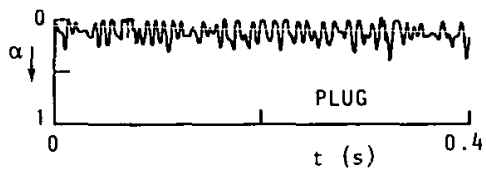

PLUG

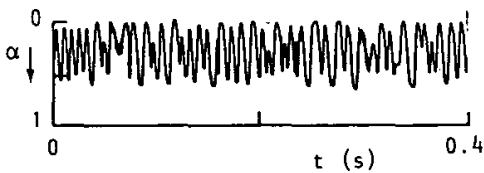

PLUG/SLUG

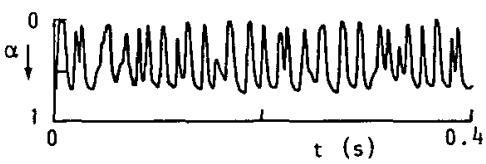

SLUG

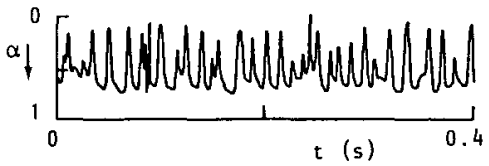

figure 6.3d: Time-varying void signals of the 50 and $5 \mathrm{~mm}$ tests, test conditions $a_{41}$ (top), $a_{42}, a_{43}$ and $a_{44}$. 
The plug frequency of the fluid velocity $a_{2}$ and $a_{3}$ increases with increasing gas velocity whereas at the velocity $a_{4}$ the revers occurs. This trend is predicted well by the model for slug frequency in horizontal pipes, developed by Taitel and Dukler (1977). The model can be applied to the stationary $50 \mathrm{~mm}$ as well as the rotating $5 \mathrm{~mm}$ section.

\section{1 .4 Conclusions}

The agreement between the results of the original and centrifugal scaled flows is very good. Except for the conditions with a stratified flow pattern the friction multiplier differs less than 19\%; at the two highest liquid velocities the difference is less even than $5 \%$. The void fraction agrees within \pm 0.03 with the exception again of the conditions with very low velocities. The time-varying void signals from which the flow pattern is deduced are similar in detail. Only in one case, is the flow pattern different.

Consequently the six dimensionless groups, the ratio of the gas and liquid velocity and the geometry of the system completely determine the flow. These results emphasize that dimensionless groups have to be used for developing correlations or comparing measurements. Nevertheless dimensional prediction methods are still in use and frequently are recommended, for example the Mandhane map (figure 1.2).

The variation (1\%) of the effective gravity over the tube and the Coriolis forces, which in some cases can be up to $15 \%$ of the effective gravitational forces (see section 2.3.2) are of negligible influence, in the flow regimes concerned.

The viscosity ratio and Euler number, which are different for the original and model flow are of minor influence (the viscosity of the gas in the model flow is actually too high, still the pressure drop at high gas flow rates is lower in the model flow). In section 6.3 the relative importance of the dimensionless groups are discussed extensively. 


\subsection{COMPARISON WITH LITERATURE}

Of the dozens of prediction methods for frictional pressure drop and void fraction published to date a few of ten cited correlations are discussed. In section 6.3 where the influence of the various dimensionless groups is reported some of the correlations will briefly be regarded again.

\section{Pressure drop}

Dukler et al (1964a) presented a critical comparison of six correlations which have found wide use, with 9000 selected experimental data. The oldest correlation, the one developed by Lockhart and Martinelli in 1949 was found to give the best overall agreement. However the correlation is far from perfect and they developed new correlations based on similarity analysis (Dukler et al 1964b). Only inertia forces and viscous shear forces are considered in their approach. For calculating the pressure drop the case I or no-slip model uses a Blasius type equation (4.1, in fact) with the following definitions of the pseudo single-phase properties: $\rho_{m}=\rho_{L} \lambda+\rho_{G}(1-\lambda), \mu_{m}=\mu_{L} \lambda+\mu_{G}(1-\lambda), U_{m}=U_{L}+U_{G}$, where $\lambda=U_{L} /\left(U_{L}+U_{G}\right)$. The model is much simpler to use than the LockhartMartinelli correlations. The case II or constant-slip model introduces a relative velocity between the phases but the ratio of the phase velocities to the averaged velocity is constant over the a cross-section. Experimental data on pressure drop has been used for constructing the correlation. The application of this correlation requires a method for the prediction of void fraction. The two correlations has been discussed by DeGance and Atherton (1970 a and b).

Mandhane et al (1977) gathered 10500 data points and compared sixteen frictional pressure drop correlations. They suggested a two-step method requiring a determination of the flow pattern (by means of the Mandhane map, 1974) and the use of a specific frictional pressure drop correlation for each flow pattern. The simple case I no-slip correlation demonstrated nearly the same accuracy as the two-step method, regardless of the flow pattern. Note that the dimensional Mandhane-map uses the superficial fluid velocities $\mathrm{U}_{\mathrm{L}}$ and $\mathrm{U}_{\mathrm{G}}$ as co-ordinates and hence in general it must fail to predict the correct flow pattern.

Recently Hewitt (1982) recommended the empirical Friedel correlation (1979) and Chrisholm correlation (1973) for calculating frictional pressure 
drop. The region in which a particular correlation should be used was defined by means of the ratio of the liquid and gas viscosity and the dimensional total mass velocity $\left(p_{L} U_{L}+p_{G} U_{G}\right)$. The results of the scaling test show that these parameters cannot be used for this purpose. The viscosity ratio $\mu_{G} / \mu_{L}$ and total mass velocity of the $5 \mathrm{~mm}$ test are respectively a factor of 3.6 and 3.0 larger than those in the $50 \mathrm{~mm}$ tests whereas the friction multiplier is the same. Because of the strong influence of the viscosity ratio in the Friedel correlation the prediction for the $5 \mathrm{~mm}$ tests are on the average $20 \%$ higher than the predictions for the $50 \mathrm{~mm}$ tests. The dimensional Chrisholm correlation (which fits the Baroczy (1965) curves), applied to the present experiments, predicts values of the friction multiplier which are in first approximation proportional to $\left(\mu_{L} / \mu_{G}\right)^{0.12}$ and inversely proportional to the square root of the mass velocity.

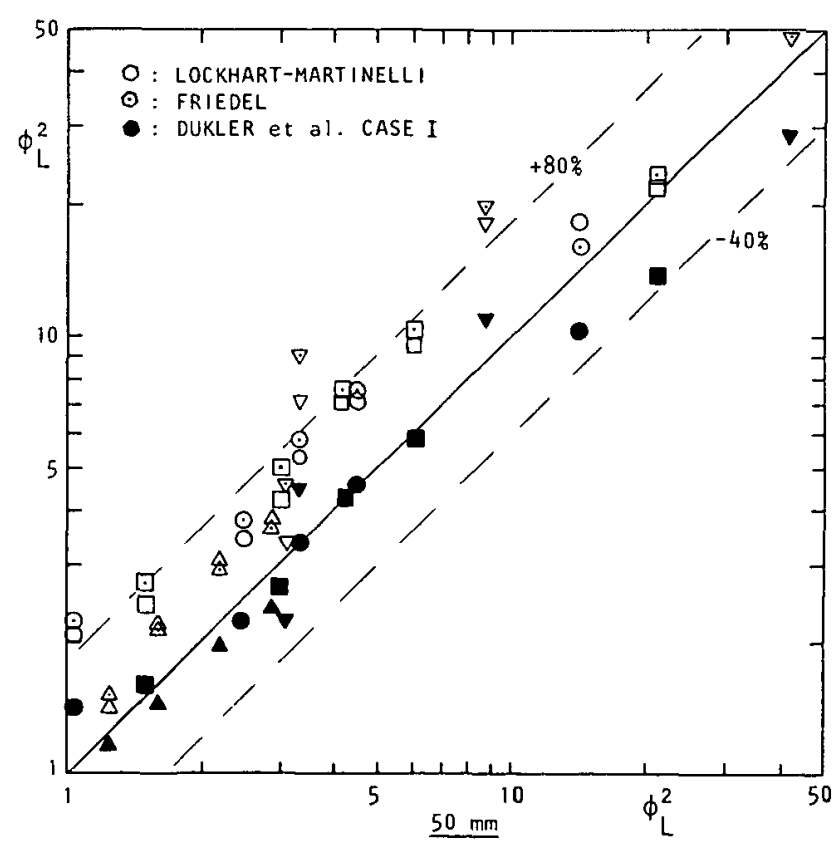

Figure 6.4: Comparison of measured (horizontal axis) and predicted (vertical axis) friction multiplier. 
The predicted values for the $5 \mathrm{~mm}$ tests are therefore the half of the values for the $50 \mathrm{~mm}$ tests.

Beattie and whalley (1982) concluded from the good performance of their simple homogeneous model that the mass velocity effect is not as significant as proposed by many investigators. They suggested that errors due to the neglect of mass velocity effects are less than those due to the neglect of other effects, such as entrance conditions.

Figure 6.4 shows a comparison of the measured friction multiplier $(50 \mathrm{~mm}$ test series $A_{1}$ ) and the predicted values of the Lockhart-Martinelli correlation, the Friedel correlation and the Dukler case I correlation.

In table 6.2 the arithmetic-mean deviation $\bar{d}=\frac{1}{n} \sum_{i=1}^{n} d_{i}$ and the standard deviation $\sigma=\left(\sum_{i=1}^{n} \frac{\left(d_{i}-\bar{d}\right)^{2}}{n-1}\right)^{0.5}$ are listed, $d_{i}=\frac{(\Delta p / \Delta L)_{\text {pred }}-(\Delta p / \Delta L)_{\text {meas }}}{(\Delta p / \Delta L)_{\text {meas }}}$

The most simple correlation, Dukler case $I$, is seen to give the best prediction. The complicated Friedel correlation shows the largest differences with the $50 \mathrm{~mm}$ data. Moreover as mentioned before the prediction of the Friedel correlation for the $5 \mathrm{~mm}$ test are $20 \%$ larger on the average. The agreement of the $5 \mathrm{~mm}$ and $50 \mathrm{~mm}$ experimental data is much better than the predictions of any correlation. Note that only the Friedel correlation allows for influences of the Froude number or Weber number. In first approximation the friction multiplier is proportional to $\mathrm{Fr}^{-0.045}$ and We -0.035 .

\begin{tabular}{|lcc|}
\hline prediction method & $\overline{\mathrm{d}} \times 100 \%$ & $\sigma \times 100 \%$ \\
\hline Lockhart-Martinelli & +52 & 32 \\
Friedel & +61 & 43 \\
Dukler case I & -6 & 21 \\
Dukler case II & +23 & 24 \\
$5 \mathrm{~mm}$, test series A & \\
$\mathrm{a}_{11}$ and $\mathrm{a}_{21}$ are not considered & -2 & 9 \\
\hline
\end{tabular}

Table 6.2: Comparison of predicted pressure drop with the $50 \mathrm{~mm}$ tests $\left(A_{1}\right)$. 


\section{Void fraction}

Figure 6.5 shows the comparison of the measured vold fraction of the 50 mm tests with the empirical correlation of Lockhart-Martinelli (1949) and the correlation of Spedding \& Chen (1984). The Lockhart-Martinelli graphic correlation curve is fitted well by the expression: $\alpha=\left(\phi_{L}-1\right) / \phi_{L}$. (Hewitt 1982). The Spedding \& Chen prediction is given by: $1 / \alpha=1.2\left(1+U_{L} / U_{G}\right)$, see also wallis (1969).

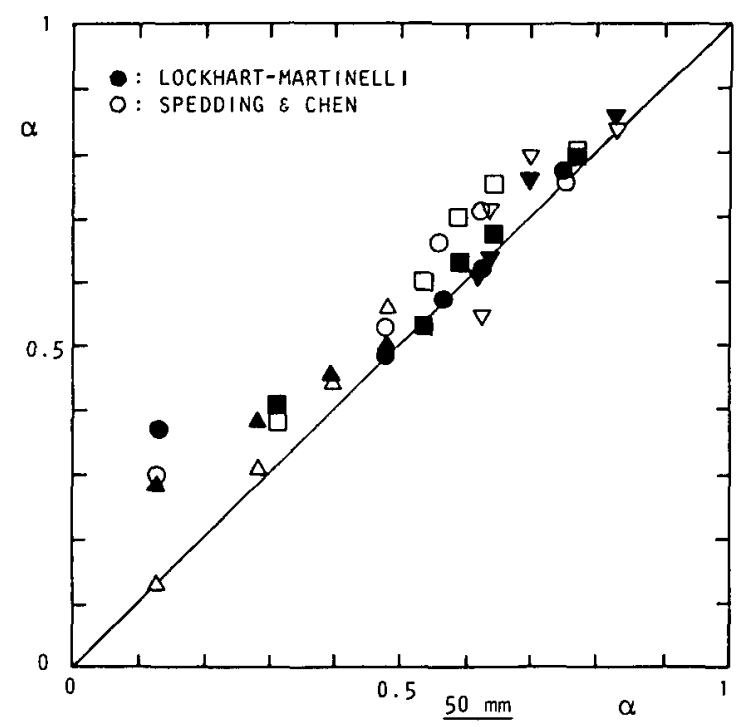

Figure 6.5: Comparison of measured $(50 \mathrm{~mm}$, test series $A_{1}$ ) and predicted vold fraction.

The arithmetic-mean deviation $\frac{1}{n} \sum_{i=1}^{n}\left(\left(\alpha_{\text {pred, } 1}-\alpha_{\text {meas, } i}\right) / \alpha_{\text {meas, } i}\right)$ equals +0.04 for the Lockhart-Martinelli correlation and +0.06 for the Spedding \& Chen correlation. The agreement of the 5 and $50 \mathrm{~mm}$ measurements (see figure 6.2) is better than the predictions of the correlation, with the exceptance of stratified flow situations. 


\section{Flow pattern}

To predict flow pattern, frequently dimensional maps are used: Baker (1954), Hoogendoorn (1959), Govier and Omer (1962), Mandhane (1974). Often the superficial velocities are used as co-ordinates and the maps depend strongly on the particular data being used to prepare them. In general dimensional prediction methods must fail to predict the flow pattern for the original and model flow. Perhaps the most promising approach is that of Taitel \& Dukler (1976). For horizontal and near horizontal flow the various transitions have been expressed in terms of dimensionless groups, essentially being $U_{G} / U_{L}$, Re, $F r, \rho_{G} / \rho_{L}$ and $\mu_{G} / \mu_{L}$ in addition to the inclination angle. In view of the semi-theoretical base and the dimensionless form of the correlation, reasonable predictions are to be expected for a wide range of experimental conditions. The measured flow pattern and the influence of the inclination angle are predicted well by this method.

\subsection{INFLUENCE OF THE DIMENSIONLESS GROUPS}

The dimensionless groups Euler, $\rho_{G} / \rho_{L}$, Reynolds, Froude and Weber can be varied separately, see section 2.4 .2 (the viscosity ratio is left out of consideration). The measurements concerning the variation of the Froude number are unique and very easy to realise in the rotating test section. Some of the results will be compared with the predicted trends of the correlations. The results and detalls concerning the experimental conditions are given in Appendix I.

\subsubsection{Influence of the Euler number}

The Euler number $E u$, defined here as $p_{G, c h a r} / \rho_{L} U_{L}^{2}$ (see equation 2.3.1, $U=U_{L}$, is of the order of the ratio of the system pressure and the two-phase pressure drop over 40 diameters ( $=$ distance between pressure taps). In the literature often the Euler number is defined as twice the friction multiplier. As can be seen from table I-1 and I-2 its value is high and hence the expansion of the gas is small over distances of interest. Hence one may expect that the different value of the Euler number for the 5 and $50 \mathrm{~mm}$ tests $\left(E u_{5} / E u_{50}=0.66\right)$ will not af fect the results. 
The Euler number is lowered by decreasing the operating pressure. At the same time the molecular weight of the gas is raised in order to keep the gas density unchanged. A mixture of helium and $14 \%$ air (test series $A_{8}$ ) and air (test series $A_{9}$ ) are used instead of helium (test series $A_{7}$ ), resulting in a reduction of the Euler number by a factor of 2 respective 7 . As can be seen from table I-8, Appendix I the friction multiplier differs less than $4 \%$ except for the test condition $a_{24}$ of the series $A_{9}$.

\subsubsection{Influence of the density ratio}

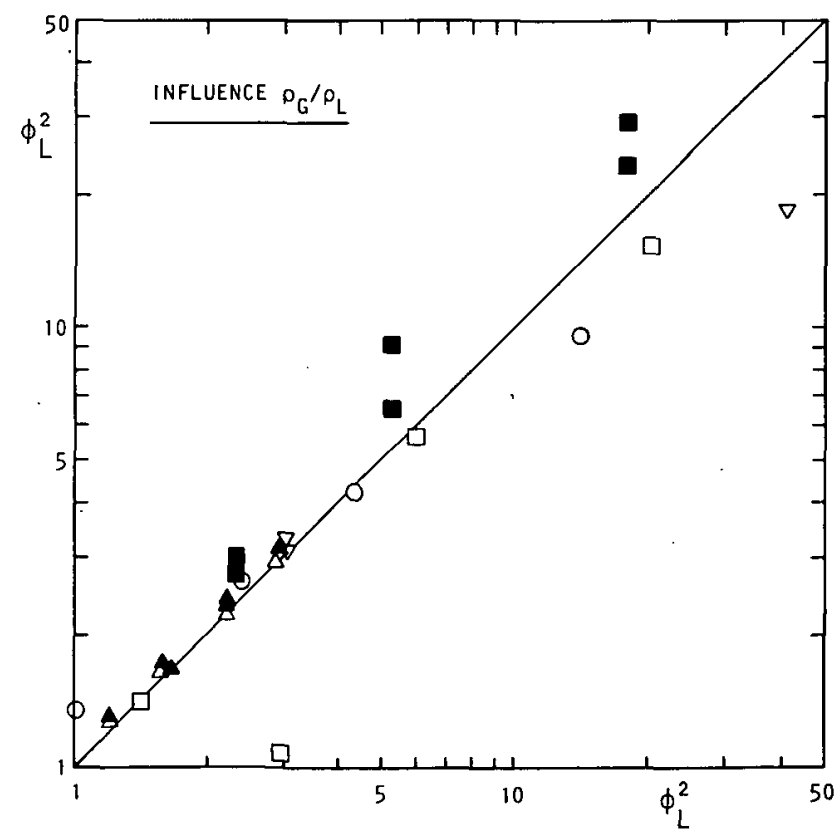

Figure 6.6: Influence of the density ratio. open symbols: $50 \mathrm{~mm}$, horizontal $A_{1}$, vertical $A_{3}$ closed symbols: $5 \mathrm{~mm}$, horizontal $A_{7}$, vertical $A_{10}$ or $A_{11}$ $\left(\rho_{G}\right)_{3} /\left(\rho_{G}\right)_{1}=0.23,\left(\rho_{G}\right)_{10} /\left(\rho_{G}\right)_{7}=1.8,\left(\rho_{G}\right)_{11} /\left(\rho_{G}\right)_{7}=7.2$.

The gas-iiquid density ratio is changed by varying the gas density. In the $5 \mathrm{~mm}$ section instead of helium (test series $A_{7}$ ) a mixture of helium and 
$14 \%$ air (test series $A_{10}$ ) and air (test series $A_{11}$ ) are used by which the density is raised by a factor of 1.8 and 7.2 . The gas density of the $50 \mathrm{~mm}$ section is lowered by a factor of 4.4 simply by decreasing the operating pressure from $0.44 \mathrm{MPa}\left(A_{1}\right)$ to atmospheric $\left(A_{3}\right)$. Only pressure drop and, for the $50 \mathrm{~mm}$ section, also flow pattern (from visual observations) are measured.

At low liquid flow rates (Indicated by the symbols $\nabla$ and $\square$ ) the influence of the density ratio is considerable: see figure 6.6. At high liquid flow rates $(\Delta)$ the effects are small. This trend is predicted well by the Dukler case I correlation.

Two data points, one close to the vertical axis and one close to the horizontal axis deviate from the general trend. The flow pattern of the test condition $a_{22}(0)$ changes from plug to stratified with an accompaning lower pressure drop. The flow pattern of the test condition $a_{31}$ (o) changes from stratified to plug. This transition is remarkable in view of the lower gas density. However as noted before the test condition $a_{31}$ is very close to a transition point and a small change of the inclination angle or vibrations of the system could trigger the transition.

\subsubsection{Influence of the Reynolds number}

In the $50 \mathrm{~mm}$ system the Reynolds number is raised by a factor of 3.1 , using water (test series $A_{5}$ ) instead of the water/glycerine mixture. In the rotating set-up the Reynolds number is raised by a factor of 2 by increasing the temperature from $20^{\circ} \mathrm{C}$ to $55^{\circ} \mathrm{C}$ (test series $\mathrm{A}_{12}$ ).

The friction multiplier is hardly affected by the increase of the Reynolds number, the values are $4 \%$ larger on the average (test conditions with a stratified flow pattern are not taken into account). The actual values of the frictional pressure drop however are considerably lower (17\% for the $5 \mathrm{~mm}$ tests and $31 \%$ for the $50 \mathrm{~mm}$ tests).

The difference between the vold fraction of the 5 and $50 \mathrm{~mm}$ tests is less than 0.01 for 29 of the 35 void fraction pairs.

In one flow situation (condition $a_{21}, 50 \mathrm{~mm}$ section) a flow pattern transition from stratified to plug is observed. The time-varying void signals of the test series with the "normal" and increased Reynolds number show 
no larger differences than those observed with repeated measurements. The slub frequencies determined from the countings of the vold peaks differ by less than $10 \%$.

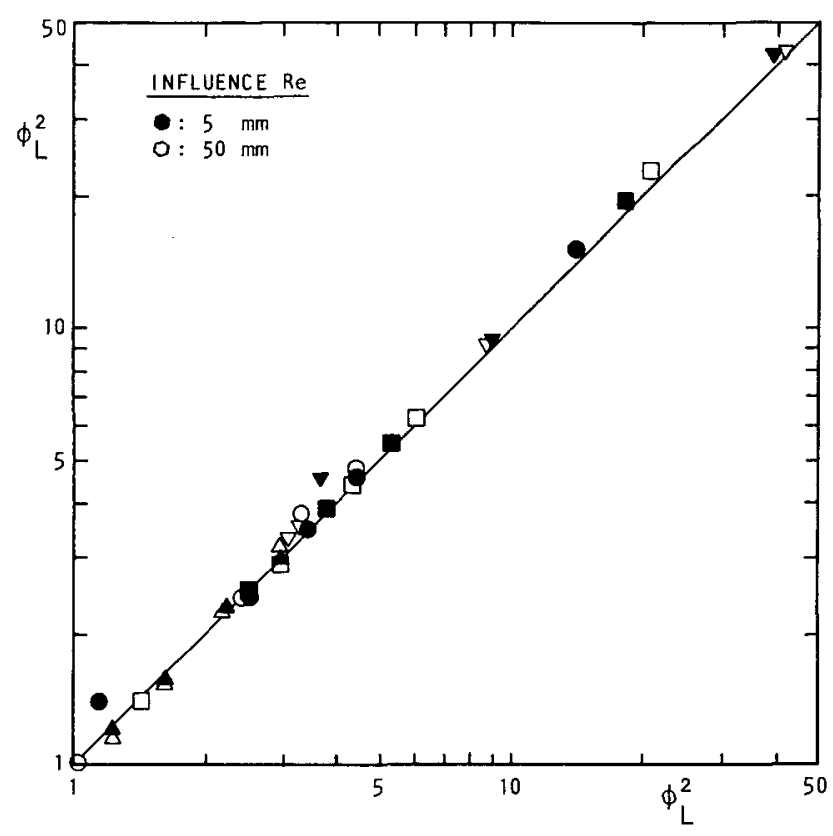

Figure 6.7: Influences of the Reynolds number. open symbols: $50 \mathrm{~mm}$, horizontal $A_{1}$, vertical $A_{5}, R_{5} / R e_{1}=3.1$ closed symbols: $5 \mathrm{~mm}$, horizontal $A_{7}$, vertical $A_{12}, R_{12} / R_{7}=2$

Taking into account the large values of the Reynolds number it is not surprising that the actual value does not affect the void fraction and the flow pattern. Only a small layer at the wall is influenced.

\subsubsection{Influence of the Froude number}

This group is varied simply by changing the rotation rate. At the same time the inclination angle of the test section is changed. As shown in section 5.2.2 the slope of the tube oniy affects the frictional pressure 
drop in stratified flow situations or in cases where a flow pattern transition is provoked. With respect to the scaling test $A_{7}$ the Froude number is raised 1.77 times (test series $A_{13}$ ) and lowered 0.46 times (test series $\left.A_{14}\right)$. In the former case only pressure drop is measured at a centrifugal acceleration of $64 \mathrm{~g}_{0}$. Five flow situations are examined, Indicated by the closed symbols in figure 6.8. At the second (a) liquid velocity the pressure drop increases $25 \%$ on the average. At the highest flow velocity ( $\Delta$ ) the differences are small.

In the second more interesting series $\left(A_{14}\right)$, pressure drop and void fraction are measured at $2458_{0}$. The pressure drop is $15 \%$ lower on the average. In $f$ ive of the flow situations the vold.fraction is decreased by more than 0.03 (maximum 0.1 at $a_{22}$, see table I-8). In these cases the largest reduction, about $30 \%$, of the pressure drop is also observed.

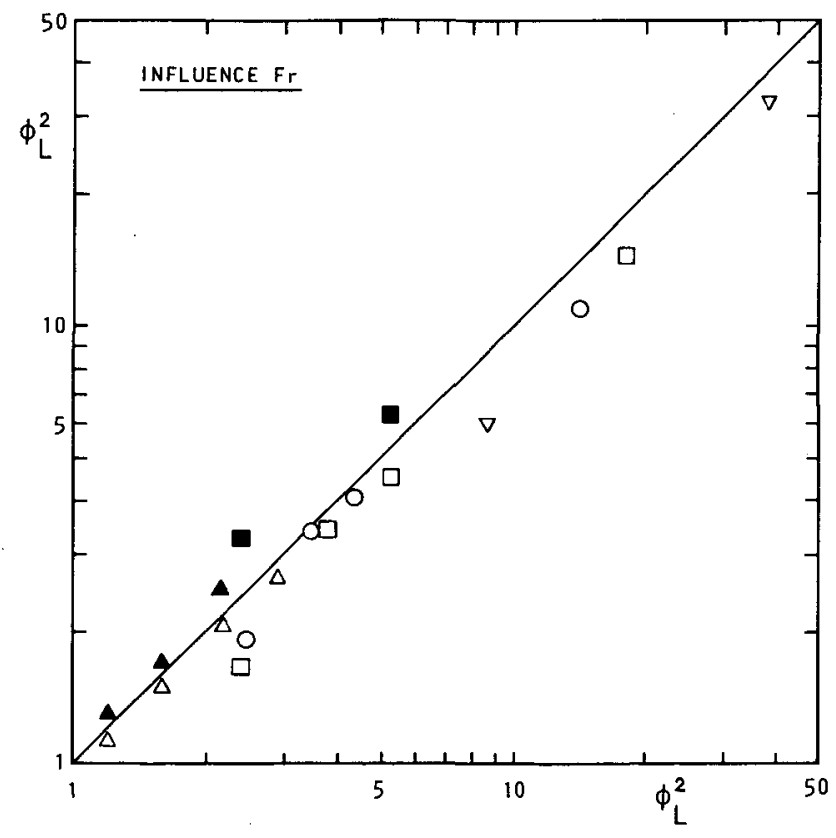

Figure 6.8: Influence of the Froude number. closed symbols: horizontal $\mathrm{A}_{7}$, vertical $\mathrm{A}_{13}, \mathrm{Fr}_{13} / \mathrm{Fr}_{7}=1.77$ open symbols: horizontal $\mathrm{A}_{7}$, vertical $\mathrm{A}_{14}, \mathrm{Fr}_{14} / \mathrm{Fr}_{7}=0.46$ 
As can be seen from figure 6.9, where the most noteworthy void signals are shown, the higher gravity results in a better separation of the phases. The flow pattern of the test condition $a_{14}$ changes from stratified wavy to stratified smooth. The plug frequency of the conditions $a_{22}, a_{32}$ and $a_{41}$ is lower whereas the length of the liquid plug is larger. The small dip in the liquid plug of condition $a_{31}$ (left hand figure) seems to result in two separated small plugs in the right hand figure. The change of intermittent flow in more stratified flow types is described by the Kelvin Helmholtz theory (see Milne-Thompson 1960). According to this theory waves formed on a flat sheet of 1 iquid flowing between two horizontal plates will grow if $U_{G}>\left(\mathrm{g} \frac{\rho_{L}-\rho_{G}}{\rho_{G}} h_{G}\right)^{0.5}$ where $U_{G}$ is the gas velocity in stratified condition and $h_{G}$ is the gas depth. The theory has been extended by Wallis and Dobson (1973) for rectangular channels and by Taitel and Dukler (1976) for channels with a circular cross-section. A higher gravity requires higher gas velocities to obtain intermittent flow.

Only a very few prediction methods for two-phase pressure drop allow for influences of the Froude number, despite its great importance. Many correlations are of the homogeneous type or are deduced by graphic methods. The Friedel correlation (1979) does incorporate the Froude number, however it predicts an increase of the pressure drop if the Froude number is decreased (increase of the gravity) whereas the measurements demonstrate the reverse relation. 
$\underline{\mathrm{Fr}_{7}}$
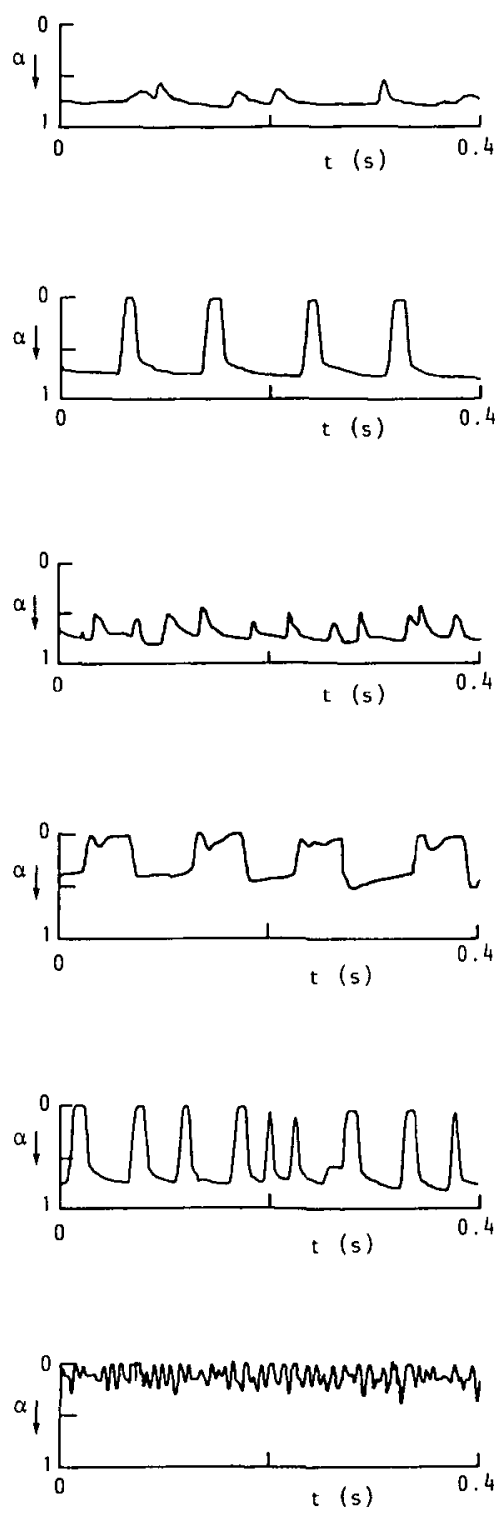
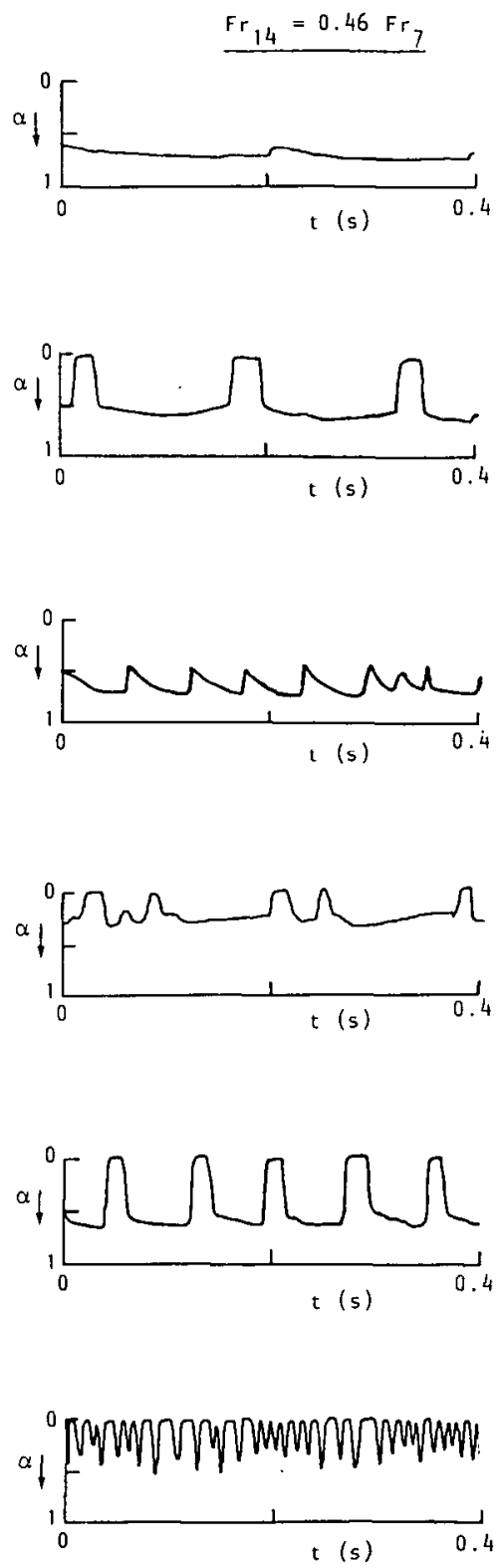

Figure 6.9: Influence of the Froude number on the time-varying void signal, test conditions $a_{14}$ (top), $a_{22}, a_{24}, a_{31}, a_{32}$ and $a_{41}$. 


\subsubsection{Influence of the Weber number}

The Weber number in the $50 \mathrm{~mm}$ tests is raised by a factor of 2.2 , using pure propanol instead of the water/glycerine mixture. The other dimensionless groups remain the same, except for the Euler number which as demonstrated is of minor influence. The surface tension of propanol is one third of the mixture value whereas the kinematic viscosity $\mu_{L} / \rho_{L}$ is the same. Because propanol causes large stresses in acrylate material the void sensor and transparent section could not be used. To prevent spreading out of the liquid in the tapping lines to the differential pressure transducer, see section 5.12, the usual $1 / 4$ inch transparent tapping lines are replaced by stainless steel tubes with an inner diameter of $1.5 \mathrm{~mm}$.

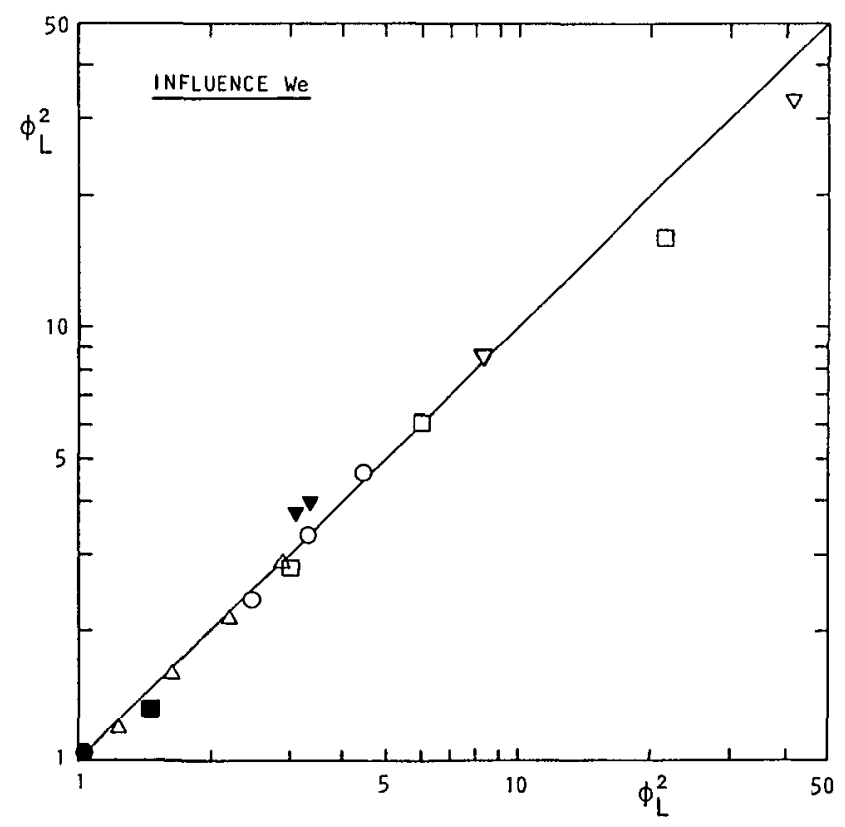

Figure 6.10: Influence of the Weber number, horizontal $A_{1}$, vertical $A_{6}$, We $6 / W e_{1}=2.2$, closed symbols: stratifled flow. 
As can be seen from figure 6.10 the influence of the weber number is small at the two largest liquid flow rates, indicated by 0 and 0 ; the differences are less than $6 \%$. At the two lowest flow rates significant differences are observed. If stratified flow pattern are not considered the decrease of the friction multiplier is $7 \%$ on the average. The reduction of the pressure drop by $23 \%$ at the largest gas velocity is remarkable. One would expect that a lower surface tension would promote droplet formation and higher pressure drops.

The flow pattern is compaired by means of the fluctuating pressure drop, see section 4.3. No clear differences are observed between the signals of the two test series. At the largest liquid flow velocity the number of peaks agree within $10 \%$, at the other flow rates within $20 \%$. At the two conditions with the largest reduction of the pressure drop also no distinctive differences could be detected. However one must recall that the pressure drop signal is less suitable for detecting differences in the flow pattern than the signal of the void sensor.

Previous studies on the influence of the Weber number or surface tension on the flow are often clouded by the presence of surface active agents. Because these substances are transported to the surface by diffusion the effects on the flow are very complicated and actually one should use.a dynamic surface tension rather than a static one (Oya 1972). Damping effects are often due to the inhomogeneous distribution of the surface active substances, not because of the reduction of the surface tension (which would faciliate formation of waves). Oya (1971) has studied the influence of surface active agents on the flow pattern in small diameter vertical tubes. The flow pattern map with the surface active solutions shows a wide difference with comparable pure liquids. v.d. Geld (1985) reported a dramatic change of the flow pattern delineation if the surface tension is suppressed ( 0.75 times), using a binary water-ethanol mixture instead of pure water. Weisman (1979) observed only a small change of the stratified wavy transition to higher gas flow rates with suppression of the surface tension by a factor of 0.55. Tomida et al (1975) reported no "appreciable" effect of surface tension on pressure drop as long as a defoaming agent is used. In vertical bubble flow the influence of the purity of the liquid (water) on the void fraction is considerable (Wallis 1969). 
It may be clear that the effects of surface active agents on the flow are complicated and experimtal results are conflicting. Here from experiments with pure 1 iquids on the average a $7 \%$ decrease of the friction multiplier is measured if the weber number is raised by a factor of 2 .

\section{3 .6 Conclusions}

Of the six dimensionless groups the Froude number and gas-liquid density ratio are the most important parameters in the flow regimes studied. Lowering of the Froude number by a factor of 2 causes significant differences in pressure drop, void fraction and flow pattern.

The Reynolds number hardly affects the void fraction and flow pattern. The actual pressure drop is approximately proportional to $\mathrm{Re}^{0.25}$ and hence the friction multiplier ( = two-phase pressure drop divided by single-phase liquid pressure drop) is nearly constant.

An increase of the Weber number causes a decrease of the pressure drop, especially at the highest gas velocity.

The gas-liquid viscosity ratio and the Euler number do not influence the pressure drop, in the flow regimes studied.

For accurate prediction of the pressure drop a correlation should thus incorporate the Froude number, the gas-liquid density ratio, the Reynolds number, the Weber number and of course the gas-liquid velocity ratio. However in many practical situations the liquids are not pure and effects from surface active agents such as foaming can be important. The Weber number effects are then overidden by those of surface tension gradients and may be neglected. If high precision is not required the Reynolds number effects can be omitted too. 


\subsection{LENGTH SCALE FACTOR OF 0.05 AND $0.5:$ THE SCALING TEST B}

The measurements of pressure drop, vold fraction and flow pattern of the tests with the $100 \mathrm{~mm}$ tube (test series $B_{1}$, horizontal flow) are compared with the results of the $50 \mathrm{~mm}$ tests (series $B_{2}$, horizontal flow) and those of the $5 \mathrm{~mm}$ tests (series $B_{4}$, centrifugal acceleration $245 \mathrm{~g}_{0}$ ). The Reynolds number, Froude number and density ratio are the same for the three tests. The weber number of the $50 \mathrm{~mm}$ and $5 \mathrm{~mm}$ tests is smaller, see table II-2, Appendix II.

\section{4 .1 Comparison of the pressure drop}

As can be seen from figure 6.11 the friction multiplier of the $50 \mathrm{~mm}$ and $5 \mathrm{~mm}$ test is larger than that of the $100 \mathrm{~mm}$ tests. For half of the examined test conditions the deviation is more than $+20 \%$. Moreover the scattering of the points is large.

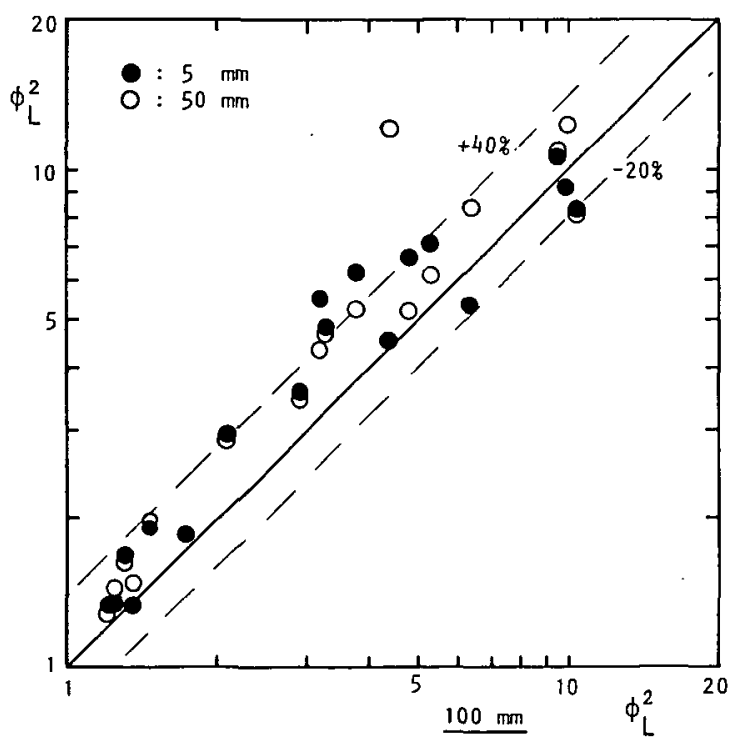

Figure 6.11: Comparison of the friction multiplier, horizontal axis: $100 \mathrm{~mm}$, test series $B_{1}$, vertical axis: $50 \mathrm{~mm}\left(B_{2}\right)$ and $5 \mathrm{~mm}\left(B_{4}\right)$. 
The comparison of the $50 \mathrm{~mm}$ tests (series $B_{3}$ ) and $5 \mathrm{~mm}$ tests (series $B_{4}$ ) shows almost a two times larger scattering than the scaling test $A$ (see Appendix II, Table II-4). The points are equally distributed around the diagonal, the arithmetic mean deviation is $+1 \%$. The Weber number of test series $B_{4}$ is a factor of 2.3 larger than that of the test series $B_{3}$. Because the measurements of the test series $B_{3}$ are carried out at atmospheric pressure the density of the gas is too low by $18 \%$. Due to the higher We-number and lower gas-liquid density ratio one would expect lower values of the friction multiplier at a few test conditions, those with a high gas velocity and low liquid velocity $\left(b_{14}, b_{24}\right.$ and $\left.b_{34}\right)$. Only $b_{14}$ shows a lower pressure drop $(-38 \%)$.

The differences and scatter are caused by (in order of importance): - The inferior accuracy of the $100 \mathrm{~mm}$ tests. The reproducibility of the fricion multiplier is certainly not better than $10 \%$, it is rather $15 \%$ or $20 \%$ : see section 5.3 .6 .

- At high gas and liquid velocities the air-gasoll flow is very foamy because of the addition of anti-static additives to the gasoli.

- The geometrical similarity is not complete. For example pressure drop is measured over 143 diameters in the $100 \mathrm{~mm}$ system, whereas for the 50 or $5 \mathrm{~mm}$ system the distances between the pressure taps is only 40 diameters.

- Because of the high operating temperature of the $5 \mathrm{~mm}$ tests the reproducibility of the friction multiplier is $\pm 8 \%$ at high liquid flow rates and $\pm 15 \%$ at the two lowest liquid flow rates. (for the scaling test $A$ the reproducibility is $\pm 5 \%$ ).

- The accuracy of the pressure drop measurement may be decreased by the so-called pumping action of enclosed gas pockets at the pressure transducer. The over pressure at the transducer is only $0.3 \mathrm{MPa}$.

- The weber number of the $50 \mathrm{~mm}$ and $5 \mathrm{~mm}$ tests is actually too low. According to the results of section 6.3 .5 the friction multiplier is then higher. 
The void fraction of the $100 \mathrm{~mm}$ tests $1 \mathrm{~s}$ on the average 0.03 larger than that of the $50 \mathrm{~mm}$ tests and 0.06 larger than that of the $5 \mathrm{~mm}$ tests, see figure 6.12. This result is not surprising because as shown in section 5.3 .3 the Neutron Back Scattering method will yleld too large values (up to +0.1 ) for the void fraction.

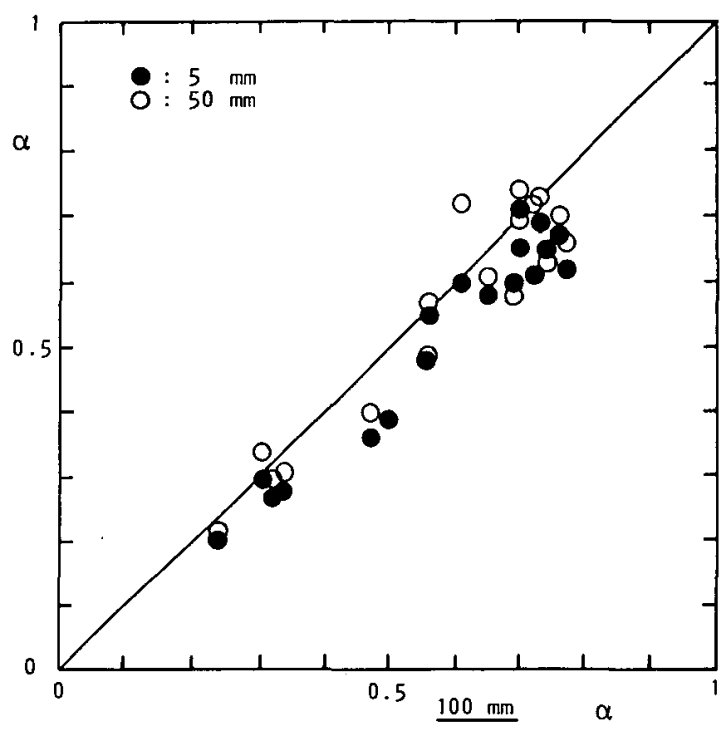

Figure 6.12: Comparison of the void fraction, horizontal: $100 \mathrm{~mm}$, test series $B_{1}$, vertical: $50 \mathrm{~mm}$, test series $B_{2}$ and $5 \mathrm{~mm}$, test series $B_{4}$.

The comparison of the $50 \mathrm{~mm}\left(B_{3}\right)$ and $5 \mathrm{~mm}\left(B_{4}\right)$ results (see Appendix II, Table II-4) shows on the average a 4\% larger vold fraction for the $5 \mathrm{~mm}$ tests at the four lowest liquid velocities. At large liquid flow rates the differences are small. 


\section{4 .3 Comparison of the flow pattern}

A comparison of the flow pattern of the $100 \mathrm{~mm}$ tests $\left(B_{1}\right)$, the $50 \mathrm{~mm}$ tests $\left(B_{2}\right.$, horizontal flow), the $50 \mathrm{~mm}$ tests $\left(B_{3}\right.$, downward inclination $1: 245)$ and the $5 \mathrm{~mm}$ tests $\left(B_{4}\right)$ yleld no large differences, see table $I-5$. All flow patterns of the $100 \mathrm{~mm}\left(B_{1}\right), 50 \mathrm{~mm}\left(B_{2}\right)$ and $5 \mathrm{~mm}\left(B_{4}\right)$ tests are intermittent (see Appendix II, Table II-4). In the $100 \mathrm{~mm}$ test more slug-type pattern are measured. However these are determined from visual observations and the distinction between plug and slug is rather subjective. For the $50 \mathrm{~mm}$ and $5 \mathrm{~mm}$ tests the time-varying signal of the void sensor is used for determining the flow pattern, see figure 6.13. An example of the measured void signals of the $100 \mathrm{~mm}$ tests is given in figure 6.14 .

If the inclination angle of the $50 \mathrm{~mm}$ test section is changed from $1: 245$ to horizontal the plug flow pattern of the test conditions $b_{13}$ and $b_{14}$ changes into stratified.

The plug/slug frequency is determined from counting of the peaks in the void signals. Eleven test conditions are used for calculating the averaged value of the frequency ratio. The result is shown in table 6.3 .

\begin{tabular}{|c|c|c|c|}
\hline & $\frac{f_{5}}{f_{100}}$ & $\frac{f_{50}}{f_{100}}$ & $\frac{f_{5}}{f_{50}}$ \\
\hline $\begin{array}{l}\text { theoretical } \\
\text { value }\end{array}$ & 73 & 1.45 & 50 \\
\hline $\begin{array}{l}\text { measured } \\
\text { value }\end{array}$ & 100 & 1.9 & 60 \\
\hline $\begin{array}{l}\text { standard } \\
\text { deviation }\end{array}$ & 15 & 0.2 & 9 \\
\hline
\end{tabular}

Table 6.3: Ratios of plug or slug frequencies.

The deviations from the theoretical values, $\mathrm{U}_{2} / \mathrm{U}_{1} \mathrm{xL}_{1} / \mathrm{L}_{2}$ ( 1 indicates the large scale flow and 2 the small scale flow) are rather high, $20 \%$ to $37 \%$. The values of table 6.2 does not differ significantly if the highest liquid flow rates, at which the countings of the void peaks are rather inexact, are not considered. 
$50 \mathrm{~mm}$ TEST SECTION
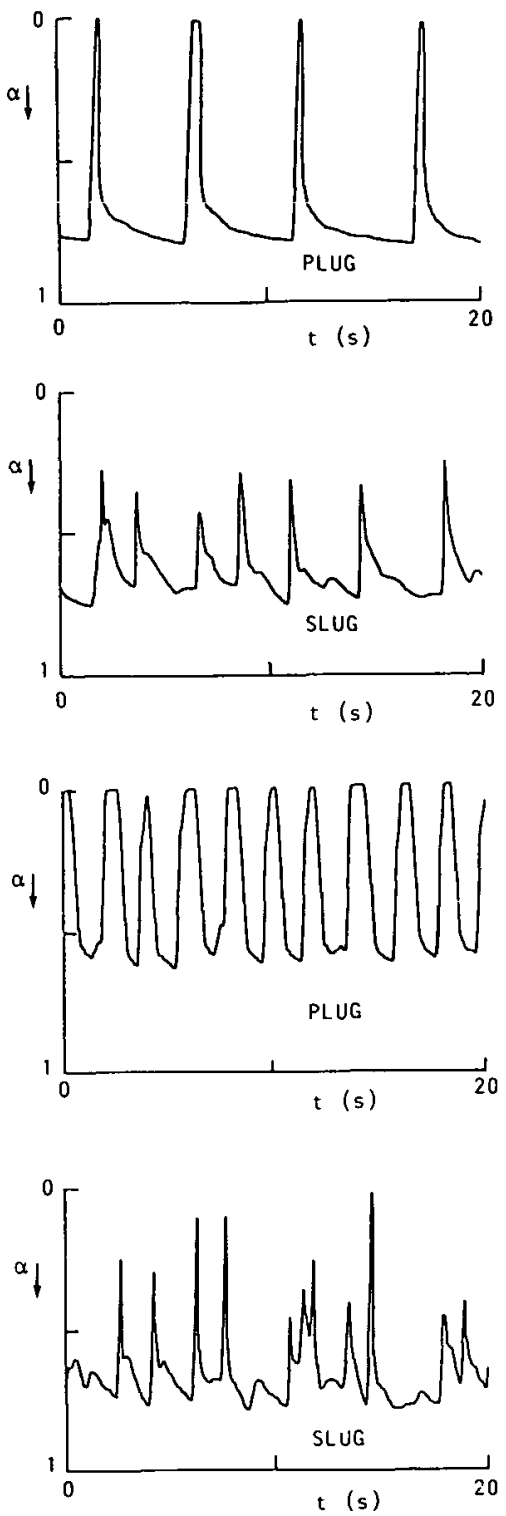

5 mII TEST SECTION
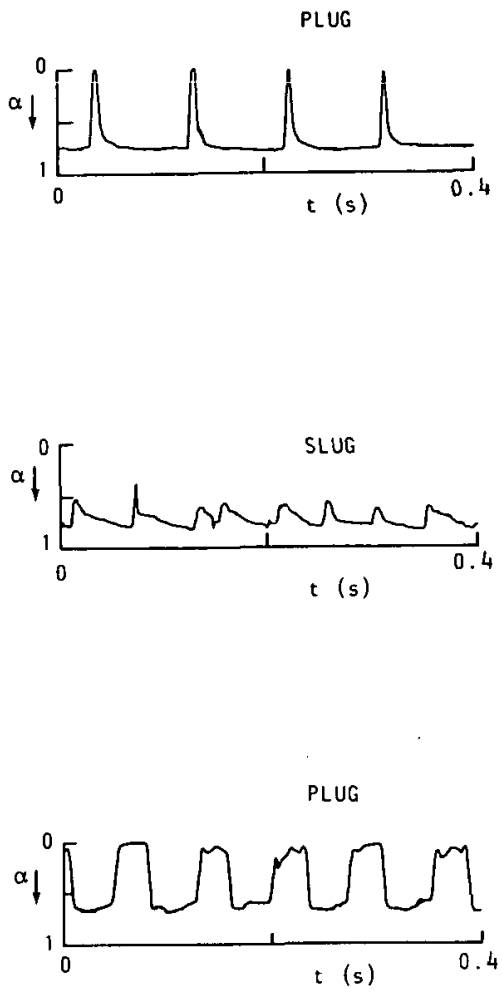

SLUG

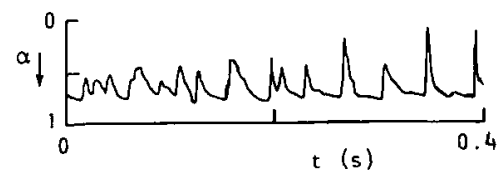

Figure 6.13: Time-varying void signals of the $50 \mathrm{~mm}\left(B_{2}\right)$ and $5 \mathrm{~mm}\left(B_{4}\right)$ tests, test conditions $b_{33}$ (top), $b_{34}, b_{41}$ and $b_{44}$. 

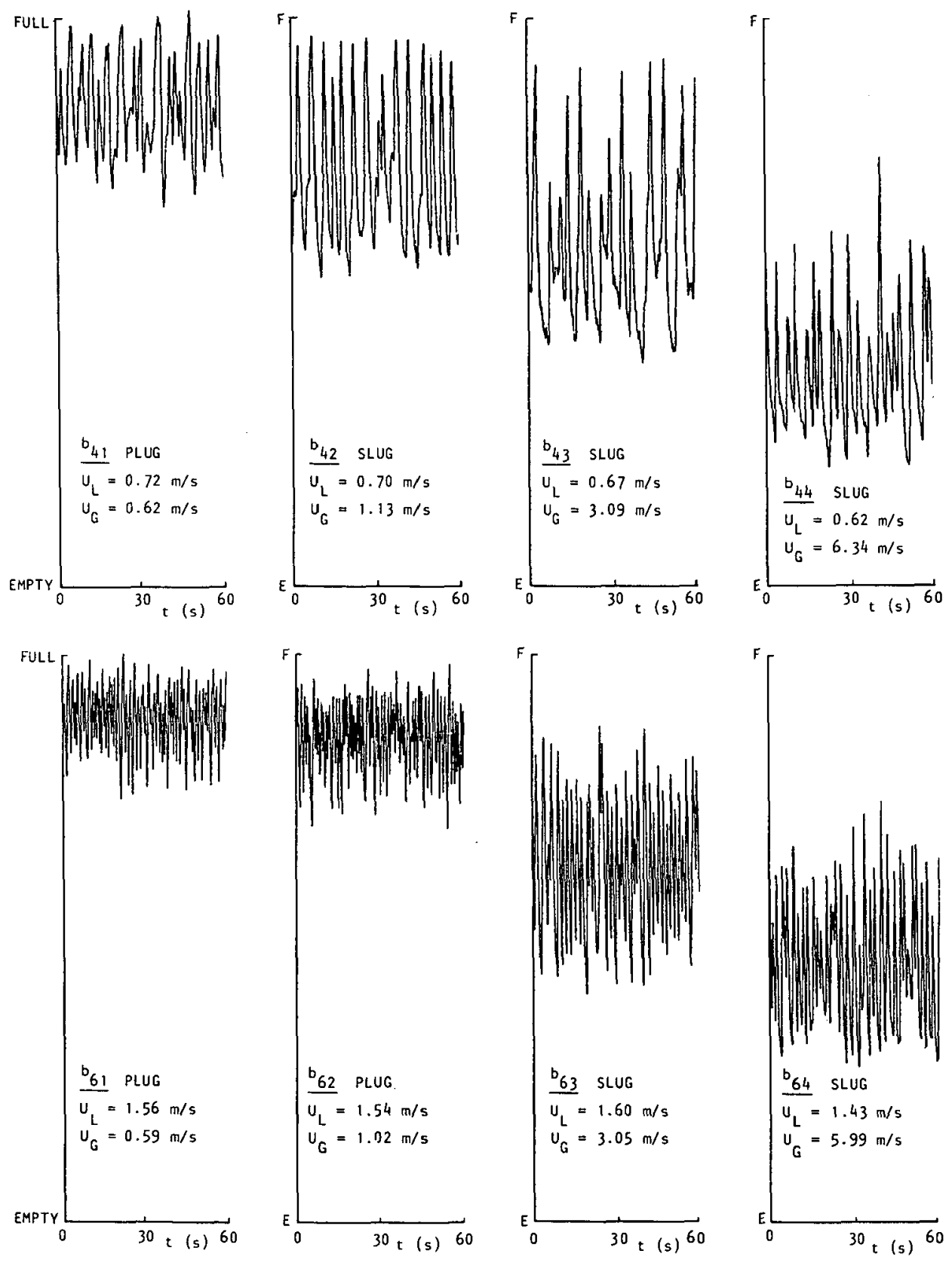

Figure 6.14: Time-varying void signals of the $100 \mathrm{~mm}$ tests. 


\section{$\underline{6.4 .4 \text { Conclusions }}$}

The agreement in the $100 \mathrm{~mm}$ results and the 50 or $5 \mathrm{~mm}$ results is poor. The differences of the pressure drop measurements are larger than $20 \%$ for half of the test data. This is mainly due to the limited accuracy of the $100 \mathrm{~mm}$ tests, as pointed out in section 5.3.2. Accurate comparisons of the vold fraction are not possible because of the relative large errors in the void fraction determinations using the neutron back scattering method. The flow pattern is intermittent in all flow conditions. The measured frequency scale factor is $34 \%$ larger than the predicted value from the similarity analysis. It may be clear that high demands have to be imposed on the accuracy of the instrumentation and performance of the experiments, for obtaining reproducible and reliable measurements.

Because of the high temperature and relative small operating pressure the agreement of the $50 \mathrm{~mm}$ and $5 \mathrm{~mm}$ results of the scaling test $B$ is less than that of the scaling test $A$. The scattering of the data around the diagonal is twice as large. 


\section{APPLICATION OF CENTRIFUGAL SCALING}

Before examples of scaling down practical flows are considered, the possibilities and restrictions of the present centrifuge facility will be discussed.

As reported in section 4.1 .4 the measurements were hindered by leakage of cooling water from the seal unit into the test circuit. Not only the filling degree of the separator was changed, but also the purity of the liquid was affected. The seal unit can be dismounted very easy and by renewing the metallised carbon stationary seal rings, see figure 4.5 , the leakage can be overcome.

A variety of liquids and gases or gas mixtures can be circulated through the test section. The only restriction is that they should not be toxic, extremely corrosive or inflammable. If necessary the nitrile o-rings can be relaced by viton or PTFE packings.

The maximum allowable operating pressure of the system is $4 \mathrm{MPa}$. Using freon $14 \mathrm{gas}\left(\mathrm{CF}_{4}\right.$, molecular weight: $88.01 \times 10^{-3} \mathrm{~kg} / \mathrm{mol}$, critical temperature: $-46^{\circ} \mathrm{C}$ ) a gas-liquid density ratio of 0.15 is realizable. To avoid under pressure in the liquid circuit and possible flashing, the hydrostatic pressure loss, $\rho_{L} \omega^{2} R^{2} / 2$, in the radial part of the rotor must always be less than the difference between the operating pressure and the saturated vapour pressure. This condition restricts the maximum rotation rate. If for example water at $20^{\circ} \mathrm{C}$ and $3 \mathrm{MPa}$ is applied, then the maximum rotation rate is about 1500 revolutions per minute $\left(=1200 \mathrm{~B}_{0}\right)$. The smaller the diameter of the rotor the smaller the hydrostatic pressure loss at a prescribed centrifugal acceleration. On the other hand the coriolis forces and the variation of the effective gravitational acceleration over the diameter of the tube are larger then.

If extra attention is paid to the fastening of the test section and care is taken to suppress vibrations of the system, a rotation rate of 1500 revolutions per minute $\left(=1200 \mathrm{~g}_{0}\right)$ is feasible.

Scaling of a pipeline containing a flow of a viscous oil and natural gas

As an example of scaling down of a large scale system, consider a horizontal pipeline of diameter $600 \mathrm{~mm}$ containing a two-phase flow of a 
viscous oil and natural gas. The density of the liquid is typically $10^{3} \mathrm{~kg} / \mathrm{m}^{3}$, its dynamic viscosity $20 \times 10^{-3} \mathrm{~Pa} . \mathrm{s}$ and the surface tension $25 \times 10^{-3} \mathrm{~N} / \mathrm{m}$. The operating pressure is $8 \mathrm{MPa}$ and the gas-liquid density ratio is 0.1 . It is supposed that a length scale down factor of $1 / 60$ is required and the feasibility of this will be examined.

The rotation rate is determined by the requirement of equality of the Froude number: $\mathrm{g}_{2} / \mathrm{g}_{1}=\left(\mathrm{U}_{2} / \mathrm{U}_{1}\right)^{2} \mathrm{~L}_{1} / \mathrm{L}_{2}$. (1 indicates the large scale system and 2 the rotating system). To obtain practicable values (< 1500 r.p.m.) of the rotation rate, the value of the velocity scale factor, required for the equality of the Reynolds number must be smaller than 5 , leading to a viscosity scale factor smaller than 0.08 . If we choose water at $20^{\circ} \mathrm{C}$ as 1 iquid scalant then the velocity scale factor becomes 3 and the required centrifugal acceleration is $540 \mathrm{~g}_{0}$. The weber number $U_{L}^{2} \rho_{L} L / \sigma$ in the model flow is then smaller by a factor of 20 . As shown in section 6.3 .5 the influence of this group can be large, especially at high gas flow rates and the results of the small scale flow are suspect then.

As pointed out at the beginning of this section a gas-liquid density ratio of 0.1 can be easily realized in the rotating system by using freon 14 gas. Compressibility and gas viscosity are not properly scaled: $\mathrm{Eu}_{2} / \mathrm{Eu}_{1} \approx 0.05$ and $\left(\mu_{G} / \mu_{L}\right)_{2} /\left(\mu_{G} / \mu_{L}\right)_{1} \approx 20$.

If a smaller length scale factor of, say $1 / 30$, is applied the required centrifugal acceleration is much lower $\left(68 \mathrm{~g}_{0}\right)$ and the weber mismatch is still larger: $W_{2} / W_{1}=0.025$. Since $B$ is relatively low however it is possible to increase $i t$, leading to larger velocities in model, (required to preserve the Froude number) and equality of the weber number. The price paid, however, is that the Reynolds number is now not matched: $R e_{2} / R e_{1}=4$. The new $\mathrm{g}$ value is $1088 \mathrm{~g}_{0}$.

\section{Scaling of a pipeline containing a flow of condensate and natural gas}

Again a horizontal pipeline of diameter $600 \cdot \mathrm{mm}$ is considered. The liquid viscosity is low now: $0.2 \times 10^{-3} \mathrm{~Pa} . \mathrm{s}$, the density is $600 \mathrm{~kg} / \mathrm{m}^{3}$ and the surface tension $2 \times 10^{-3} \mathrm{~N} / \mathrm{m}$. A length scale factor of $1 / 30$ will be assumed. Unless the viscosity of the scalant liquid is very low (of order $0.1 \times 10^{-3}$ Pa.s) the required centrifugal acceleration becomes very large. A possible scalant liquid is freon $12\left(\mathrm{CCl}_{2} \mathrm{~F}_{2}, \mu_{\mathrm{L}}=0.26 \times 10^{-3} \mathrm{~Pa} . \mathrm{s}\right.$ at $25^{\circ} \mathrm{C}$ and the saturated vapour pressure is $0.65 \mathrm{Mpa}$ ). By increasing the temperature the 
viscosity can be lowered still further. However heat and mass transfer will then no longer be negligible. If we assume that the density, viscosity and surface tension of the scalant liquid are $1200 \mathrm{~kg} / \mathrm{m}^{3}, 0.1 \times 10^{-3} \mathrm{~Pa} . \mathrm{s}$ and $4 \times 10^{-3} \mathrm{~N} / \mathrm{m}$ then the velocity scale factor becomes 7.5 , the required centrifugal acceleration $1700 \mathrm{~g}_{0}$ and the ratio of the weber number is 1 . To avoid flashing in the liquid circuit the operating pressure should be at least $7 \mathrm{MPa}$. At this pressure high gas-liquid density ratios are easy to realise. The Euler number is too low by a factor of 100 , whereas the gas-liquid viscosity ratio will be of the same order in both flows.

If a deviation of the Reynolds number and Weber number by a factor of 5 is accepted then the experimental conditions are much more practicable: the centrifugal acceleration becomes $425 \mathrm{~g}_{0}$ (at the same time lowering the velocity by a factor of 2 to preserve the Froude number), the operating pressure can be $3 \mathrm{MPa}$ and the temperature $25^{\circ} \mathrm{C}$.

At constant gravity often only one criterion, the modified Froude number, $\rho_{G} / \rho_{L} x^{2} / g L$ is used for scaling purposes. This group is also an important parameter in the Taitel and Dukler approach (1976) for predicting flow pattern transitions. If the modified Froude number is used here instead of the gas-liquid density ratio and the usual Froude number, the situation becomes much more flexible. Lower density ratios can then be combined with lower centrifugal accelerations and lower pressures and temperatures. Moreover the choice of the liquid and gas scalant would become much easier.

Summarizing: large diameter, high pressure systems can be scaled down to laboratory dimensions, by choosing proper liquid and gas scalants, centrifugal accelerations and operating pressures. With a length scale factor of 1/30 equality of the Froude number and gas-liquid density ratio can always be realized. In many cases also equality of either the Reynolds number or the weber number is feasible. Compressibility and gas viscosity are not scaled in general. 


\section{FINAL CONCLUSIONS}

A facility has been built which enables accurate two-phase measurements of pressure drop, void fraction and flow pattern, in small diameter pipes at gravities up to 1200 times natural gravity, offering the possibility of scaling-down of large horizontal flows, while preserving the dimensionless groups derived from the basic equations.

In order to check the validity of the similarity criteria for isothermal flows, measurements in a stationary $50 \mathrm{~mm}$ diameter tube and a rotating $5 \mathrm{~mm}$ tube are compared. Of elghteen examined test conditions the flow pattern is the same in all but one case, which is close to a transition. The dimensionless plug/slug frequencies agree extremely well. If stratified flow patterns are not considered the friction multiplier is the same within $19 \%$ and the void fraction within 0.03 (absolute units).

At low fluid velocities with a stratified flow pattern the measurements are less accurate due to the tolerance in the position of the test section with respect to the rotation axis and probably in some cases due to back flow of liquid from the separator.

From these and other results it became clear that the excellent agreement can only be achieved if much attention is pald to the performance of the experiments: regulary checks of the instruments, clean liquids, constant temperature and pressure, accurate adjustment of the flow velocities etc.

Within the estimated error it is concluded that a two-phase flow of a gas and a liquid is indeed determined by the values of the six dimensionless groups: the Reynolds number, the Froude number, the Weber number, the gas-liquid density ratio, the viscosity ratio and the Euler number, in addition to the geometry of the system and the gas-liquid velocity ratio. The Froude number and density ratio appeared to be the most important in the flow regime concerned, while the Euler number (representing the expansion of the gas) and the viscosity of the gas are of negligible influence.

A decrease in the Froude number by a factor of 2 results in a better separation of the phases, lower pressure drops and lower void fractions. An increase of the Weber number by a factor of 2.2 causes a decrease of the pressure drop, especially at high gas flow rates. The influence of the 
Reynolds number on the flow pattern and vold fraction is small, compared with those of the other groups. The change of the pressure drop is described well by a Blasius-type relation.

The preceding results emphasize that in general only dimensionless correlations will provide meaningful predictions. None of the current dimensionless correlations for two-phase pressure drop is particularly accurate, standard deviations of $40 \%$ are commonly encountered. This results was confirmed by the inability of such correlations to predict the present flows adequately. Surprisingly a correlation which contains no two-phase information (Dukler case I, 1946b) gives the best fit with the experimental data. On the other hand it is not astounding then that large standard deviations appear: no entrance effects or influences of specific two-phase parameters like the Froude or Weber number are incorporated in the correlation.

The principle of centrifugal scaling can be used for scalling down large diameter, high pressure two-phase flows. For example a flow of a viscous oil and natural gas in a $600 \mathrm{~mm}$ diameter pipeline can be scaled-down to a $20 \mathrm{~mm}$ diameter tube with a flow of water and freon 14 gas. The Froude number, the gas-liquid density ratio, and if a difference of the Reynolds number by a factor of 4 is accepted then also the Weber number can be equalized in both flows. Compressibility and gas viscosity are not properly scaled. 


\section{APPENDIX I}

\section{TEST CONDITIONS AND EXPERIMENTAL RESULTS OF THE SCALING TEST A}

To test the similarity criteria the measurements of the stationary test section (series $A_{1}, D=49.8 \mathrm{~mm}$ ) are compared with those of the rotating test section (series $A_{7}, D=4.90 \mathrm{~mm}$ ). In table $I-1$ and $I-2$ the superfical liquid velocity, $U_{L}$, the superficial gas velocity $U_{G}$, the single-phase liquid pressure drop and the dimensionless numbers Re, Fr, Eu and We are tabulated for each test condition, denoted by the symbol $a_{* *}$. The first subscript indicates the adjusted liquid velocity, the second one the adjusted gas velocity. The higher the number, the higher the velocity.

The fluid properties and the values of the system parameters are listed in table I-4 for the stationary test section and in table I-7 for the rotating test section.

The experimental results of the friction multiplier $\phi_{L}^{2}$, the overall vold fraction and the flow pattern are listed in table I-5 (test series $A_{1}$ ) and table $I-8$ (test series $A_{7}$ ).

For the stationary test section as well as the rotating test section fluid properties and system parameters are varied to investigate the influence of the various dimensionless groups separately.

For the stationary (rotating) test section the relative velocity and the values of the relative dimensionless groups with respect to the values of series $A_{1}\left(A_{7}\right)$ are given in table $I-2$ (table $I-6$ ).

Fluid properties and system parameters can be found in table I-4 and table I-7.

The experimental results are given in table $I-5$ and table $I-8$. To obtain the friction multiplier for the test series $A_{3}$ and $A_{6}$ the measured void fraction of series $A_{1}$ is used; for the test series $A_{8}, A_{9}, A_{10}, A_{11}$ and $A_{13}$ the void fraction of series $A_{7}$ is used. 


\begin{tabular}{|c|c|c|c|c|c|c|c|}
\hline $\begin{array}{l}\text { TEST } \\
\text { COND }\end{array}$ & $\mathrm{U}_{\mathrm{L}}$ & $\mathrm{U}_{\mathrm{G}}$ & $\left.\frac{\Delta \mathrm{P}}{\Delta \mathrm{L}}\right]_{\mathrm{L}}$ & $\operatorname{Re}$ & $\mathrm{Fr}$ & Eu & We \\
\hline & $\mathrm{m} / \mathrm{s}$ & $\mathrm{m} / \mathrm{s}$ & $\mathrm{Pa} / \mathrm{m}$ & -- & $=$ & -- & -- \\
\hline$a_{11}$ & 0.175 & 0.32 & 15 & 2840 & 0.063 & 13110 & 23 \\
\hline$a_{12}$ & 0.175 & 1.02 & 15 & 2840 & 0.063 & 13110 & 23 \\
\hline$a_{14}$ & 0.175 & 3.49 & 15 & 2840 & 0.063 & 13110 & 23 \\
\hline$a_{15}$ & 0.175 & 10.5 & 15 & 2840 & 0.063 & 13110 & 23 \\
\hline$a_{21}$ & 0.392 & 0.32 & 60 & 6400 & 0.314 & 2610 & 117 \\
\hline$a_{22}$ & 0.392 & 1.02 & 60 & 6400 & 0.314 & 2610 & 117 \\
\hline$a_{23}$ & 0.392 & 2.13 & 60 & 6400 & 0.314 & 2610 & 117 \\
\hline$a_{24}$ & 0.392 & 3.49 & 60 & 6400 & 0.314 & 2610 & 117 \\
\hline$a_{25}$ & 0.392 & 10.5 & 60 & 6400 & 0.314 & 2610 & 117 \\
\hline$a_{31}$ & 0.568 & 0.32 & 114 & 9200 & 0.66 & 1240 & 247 \\
\hline$a_{32}$ & 0.568 & 1.02 & 114 & 9200 & 0.66 & 1240 & 247 \\
\hline$a_{33}$ & 0.568 & 2.13 & 114 & 9200 & 0.66 & 1240 & 247 \\
\hline$a_{34}$ & 0.568 & 3.49 & 114 & 9200 & 0.66 & 1240 & 247 \\
\hline$a_{35}$ & 0.568 & 10.5 & 114 & 9200 & 0.66 & 1240 & 247 \\
\hline$a_{41}$ & 1.68 & 0.32 & 763 & 27300 & 5.79 & 142 & 2163 \\
\hline$a_{42}$ & 1.68 & 1.02 & 763 & 27300 & 5.79 & 142 & 2163 \\
\hline$a_{43}$ & 1.68 & 2.13 & 763 & 27300 & 5.79 & 142 & 2163 \\
\hline$a_{44}$ & 1.68 & 3.49 & 763 & 27300 & 5.79 & 142 & 2163 \\
\hline
\end{tabular}

Table I-1: Superficial liquid velocity, superficial gas velocity, single-phase liquid pressure drop and values of the dimensionless groups for the test conditions of series $A_{1}$ (stationary $50 \mathrm{~mm}$ test section). Standard symbols: $a_{11}-a_{15}: \nabla$ or $\nabla$

$$
\begin{aligned}
& a_{22}-a_{25}: 0 \text { or } \\
& a_{31}-a_{35}: \text { or } \bullet \\
& a_{41}-a_{44}: \Delta \text { or } \Delta
\end{aligned}
$$




\begin{tabular}{|c|c|c|c|c|c|c|c|}
\hline $\begin{array}{l}\text { TEST } \\
\text { COND }\end{array}$ & $U_{L}$ & $U_{G}$ & {$\left[\frac{\Delta \mathrm{P}}{\Delta \mathrm{L}}\right]_{\mathrm{L}}$} & $\operatorname{Re}$ & $\mathrm{Fr}$ & $\mathrm{Eu}$ & We \\
\hline & $\mathrm{m} / \mathrm{s}$ & $\mathrm{m} / \mathrm{s}$ & $\mathrm{Pa} / \mathrm{m}$ & -- & -- & -- & - \\
\hline$a_{11}$ & 0.58 & 1.07 & 1480 & 2840 & 0.063 & 8640 & 22 \\
\hline$a_{12}$ & 0.58 & 3.36 & 1480 & 2840 & 0.063 & 8640 & 22 \\
\hline$a_{14}$ & 0.58 & 11.6 & 1480 & 2840 & 0.063 & 8640 & 22 \\
\hline$a_{15}$ & 0.58 & 34.9 & 1480 & 2840 & 0.063 & 8640 & 22 \\
\hline$a_{21}$ & 1.29 & 1.07 & 6060 & 6400 & 0.314 & 1750 & 113 \\
\hline$a_{22}$ & 1.29 & 3.36 & 6060 & 6400 & 0.314 & 1750 & 113 \\
\hline$a_{23}$ & 1.29 & 7.06 & 6060 & 6400 & 0.314 & 1750 & 113 \\
\hline$a_{24}$ & 1.29 & 11.6 & 6060 & 6400 & 0.314 & 1750 & 113 \\
\hline$a_{25}$ & 1.29 & 34.9 & 6060 & 6400 & 0.314 & 1750 & 113 \\
\hline$a_{31}$ & 1.88 & 1.07 & 11600 & 9200 & 0.66 & 820 & 238 \\
\hline$a_{32}$ & 1.88 & 3.36 & 11600 & 9200 & 0.66 & 820 & 238 \\
\hline$a_{33}$ & 1.88 & 7.06 & 11600 & 9200 & 0.66 & 820 & 238 \\
\hline$a_{34}$ & 1.88 & 11.6 & 11600 & 9200 & 0.66 & 820 & 238 \\
\hline$a_{35}$ & 1.88 & 34.9 & 11600 & 9200 & 0.66 & $820^{\circ}$ & 249 \\
\hline$a_{41}$ & 5.56 & 1.07 & 77700 & 27300 & 5.79 & 94 & 2098 \\
\hline$a_{42}$ & 5.56 & 3.36 & 77700 & 27300 & 5.79 & 94 & 2098 \\
\hline$a_{43}$ & 5.56 & 7.06 & 77700 & 27300 & 5.79 & 94 & 2098 \\
\hline$a_{44}$ & 5.56 & 11.6 & 77700 & 27300 & 5.79 & 94 & 2098 \\
\hline
\end{tabular}

Table I-2: Superficial liquid velocity, superficial gas velocity, single-phase liquid pressure drop and values of the dimensionless groups for the test conditions of series $A_{7}$ (rotating $5 \mathrm{~mm}$ test section).

Standard symbols: $a_{11}-a_{15}: \nabla$ or $\nabla$

$$
\begin{aligned}
& a_{22}-a_{25}: \text { or } \\
& a_{31}-a_{35}: \text { o or } \bullet \\
& a_{41}-a_{44}: \Delta \text { or } 4
\end{aligned}
$$




\begin{tabular}{|c|c|c|c|c|c|c|c|c|c|}
\hline $\begin{array}{l}\text { TEST } \\
\text { SERIES }\end{array}$ & $\frac{U}{(U)_{A_{1}}}$ & $\frac{\mathrm{Re}}{(\operatorname{Re})_{\mathrm{A}_{1}}}$ & $\frac{F r}{(\overline{F r})}{ }_{A_{1}}$ & $\frac{E u}{(\overline{E u})_{A_{1}}}$ & $\frac{W e}{(W e)_{A_{1}}}$ & $\frac{\rho_{G} / \rho_{L}}{\left.\rho_{G} / \rho_{L}\right)_{A}}$ & DECL & \multicolumn{2}{|c|}{$\begin{array}{l}\text { NUMBER OF } \\
\text { COND DATA }\end{array}$} \\
\hline$A_{1}$ & 1.00 & 1.00 & 1.00 & 1.00 & 1.00 & 1.00 & $1: 113$ & 18 & 74 \\
\hline $\mathrm{A}_{2}$ & 1.00 & 1.00 & 1.00 & 1.00 & 1.00 & 1.00 & horiz & 12 & 18 \\
\hline $\mathrm{A}_{3}$ & 1.00 & 1.00 & 1.00 & 0.23 & 1.00 & 0.23 & $1: 113$ & 15 & 24 \\
\hline$A_{4}$ & 1.00 & 1.00 & 1.00 & 0.23 & 1.00 & 0.23 & noriz & 14 & 17 \\
\hline$A_{5}$ & 1.00 & 3.06 & 1.00 & 1.00 & 0.89 & 1.00 & $1: 113$ & 17 & 32 \\
\hline$A_{6}$ & 1.00 & 1.00 & 1.00 & 1.00 & 2.20 & 1.00 & $1: 113$ & 16 & 22 \\
\hline $\mathrm{A}_{7}$ & 3.33 & 1.00 & 1.00 & 0.66 & 0.97 & 1.00 & $\frac{\mathrm{g}}{\mathrm{g}_{0}}=113$ & 18 & 107 \\
\hline
\end{tabular}

Table I-3: Relative velocity and relative dimensionless numbers with respect to the series $A_{1}$ for $s i x$ test series of the stationary $50 \mathrm{~mm}$ test section and one test series $\left(A_{7}\right)$ of the rotating $5 \mathrm{~mm}$ test section.

\begin{tabular}{|c|c|c|c|c|c|c|c|c|c|}
\hline $\begin{array}{l}\text { TEST } \\
\text { SER IES }\end{array}$ & $\begin{array}{c}\rho_{L} \\
\mathrm{~kg} / \mathrm{m}^{3}\end{array}$ & $\begin{array}{c}\mu_{L} \\
\mathrm{~Pa} \cdot \mathrm{s} \\
10^{-3}\end{array}$ & $\begin{array}{c}\sigma \\
\mathrm{N} / \mathrm{m} \\
10^{-3}\end{array}$ & $\begin{array}{c}{ }^{P_{G}} \\
\mathrm{~kg} / \mathrm{m}^{3}\end{array}$ & $\begin{array}{c}{ }_{G} \\
\mathrm{~Pa} \cdot \mathrm{s} \\
10^{-6}\end{array}$ & $\begin{array}{c}\mathrm{p} \\
\mathrm{Pa} \\
10^{6}\end{array}$ & $\begin{array}{l}\mathrm{T} \\
{ }^{\circ} \mathrm{C}\end{array}$ & DECL & FLUIDA \\
\hline$A_{1}$ & 1093 & 3.35 & 71.2 & 5.22 & 18.4 & 0.439 & 20 & $1: 113$ & $\begin{array}{l}\text { water } \\
38 \% \text { glyc } / \text { in }\end{array}$ \\
\hline $\mathrm{A}_{2}$ & 1093 & 3.35 & 71.2 & 5.22 & 18.4 & 0.439 & 20 & noriz & " \\
\hline$A_{3}$ & 1093 & 3.35 & 71.2 & 1.20 & 18.4 & 0.101 & 20 & $1: 113$ & $n$ \\
\hline $\mathrm{A}_{4}$ & 1093 & 3.35 & 71.2 & 1.20 & 18.4 & 0.101 & 20 & noriz & $n$ \\
\hline $\mathrm{A}_{5}$ & 998 & 1.00 & 72.8 & 4.76 & 18.4 & 0.401 & 20 & $1: 113$ & water/air \\
\hline$A_{6}$ & 808 & 2.47 & 24 & 3.85 & 18.4 & 0.320 & 16 & $1: 113$ & propanol/air \\
\hline
\end{tabular}

Table I-4: Fluid properties and system parameters for the six test series of the stationary $50 \mathrm{~mm}$ test section. 


\begin{tabular}{|c|c|c|c|c|c|c|c|c|c|c|c|c|c|c|}
\hline \multirow[t]{2}{*}{ COND } & \multicolumn{6}{|c|}{ FRICTION MULTIPLIER } & \multicolumn{2}{|c|}{ VOID FRACT } & \multicolumn{6}{|c|}{ FLOW PATTERN } \\
\hline & $A_{1}$ & $A_{2}$ & $A_{3}$ & $A_{4}$ & $A_{5}$ & $A_{6}$ & $A_{1}$ & $A_{5}$ & $A_{1}$ & $A_{2}$ & $\mathrm{~A}_{3}$ & $\mathrm{~A}_{4}$ & $A_{5}$ & $A_{6}$ \\
\hline$a_{11}$ & 3.1 & 0.82 & 3.1 & 1.19 & 3.3 & 3.7 & 0.62 & 0.62 & SS & SS & SS & PL & SS & SS \\
\hline$a_{12}$ & 3.3 & 4.58 & 3.1 & 4.1 & 3.5 & 3.9 & 0.63 & 0.63 & SS & PL & SS & PL & SS & SS \\
\hline$a_{14}$ & 8.7 & 9.7 & - & 8.4 & 9.2 & 8.8 & 0.70 & 0.70 & SW & SL & SW & SL & SW & SW \\
\hline$a_{15}$ & 41.2 & - & 18.6 & 19.0 & 43.0 & 32.0 & 0.83 & 0.83 & $S A$ & -- & SL & SL & SA & SA \\
\hline$a_{21}$ & 1.46 & 1.52 & 1.43 & 1.61 & 1.40 & 1.30 & 0.31 & 0.35 & ss & PL & SS & PL & PL & SS \\
\hline$a_{22}$ & 3.00 & 3.11 & 1.08 & 3.10 & 2.91 & 2.74 & 0.53 & 0.53 & PL & $\mathrm{PL}$ & SS & PL & PL & PL \\
\hline$a_{23}$ & $4 \cdot 3$ & - & - & - & 4.4 & - & 0.59 & 0.61 & PS & -- & -- & -- & PS & -- \\
\hline$a_{24}$ & 6.0 & 6.0 & 5.7 & 6.0 & 6.3 & 5.9 & 0.64 & 0.64 & SL & SL & SL & SL & SL & SL \\
\hline$a_{25}$ & 20.7 & - & 15.2 & 15.6 & 22.9 & 15.8 & 0.77 & 0.77 & SA & -- & SL & SL & $\mathrm{SA}$ & SA \\
\hline$a_{31}$ & 1.03 & 1.44 & 1.35 & 1.45 & 1.00 & 1.04 & 0.13 & 0.19 & SS & PL & PL & PL & SS & SS \\
\hline$a_{32}$ & 2.45 & 2.45 & 2.55 & 2.51 & 2.44 & 2.32 & 0.48 & 0.48 & PL & PL & PL & PL & PL & PL \\
\hline$a_{33}$ & 3.30 & - & - & - & 3.78 & 3.27 & 0.56 & 0.57 & PS & -- & -- & -- & PS & PS \\
\hline$a_{34}$ & 4.4 & 4.3 & 4.2 & 4.2 & 4.9 & 4.6 & 0.62 & 0.62 & SL & SL & SL & SL & SL & SL \\
\hline$a_{35}$ & 14.2 & - & 9.2 & - & - & - & 0.75 & - & $S A$ & -- & SL & -- & -- & - \\
\hline$a_{41}$ & 1.23 & 1.29 & 1.28 & 1.29 & 1.16 & 1.18 & 0.13 & 0.13 & PL & PL & PL & PL & PL & PL \\
\hline$a_{42}$ & 1.61 & 1.65 & 1.66 & 1.66 & 1.62 & 1.55 & 0.28 & 0.29 & PL & PL & PL & PL & PL & PL \\
\hline$a_{43}$ & 2.22 & 2.25 & 2.22 & 2.23 & 2.33 & 2.08 & 0.40 & 0.41 & PL & PL & PS & PS & $\mathrm{PL}$ & PS \\
\hline$a_{44}$ & 2.94 & - & 2.94 & - & 3.14 & 2.80 & 0.48 & 0.48 & PS & -- & SL & -- & PS & PS \\
\hline
\end{tabular}

Table I-5: Experimental results of the stationary $50 \mathrm{~mm}$ test section.

Flow pattern:SS = Stratified Smooth

$$
\begin{aligned}
& \text { SW = Stratified Wavy } \\
& \text { PL }=\text { Plug, SL = Slug } \\
& \text { PS }=\text { Plug or Slug } \\
& \text { SA = Semi Annular }
\end{aligned}
$$

$$
\text { Standard Symbols: } \begin{array}{r}
a_{11}-a_{15}: \nabla \text { or } \nabla \\
a_{21}-a_{25}: \text { o or } . \\
a_{31}-a_{35}: \text { o or } \\
a_{41}-a_{44}: \Delta \text { or } \Delta
\end{array}
$$




\begin{tabular}{|c|c|c|c|c|c|c|c|c|c|}
\hline $\begin{array}{l}\text { TEST } \\
\text { SERIES }\end{array}$ & $\frac{\mathrm{U}}{(\mathrm{U})}{ }_{\mathrm{A}_{7}}$ & $\frac{\mathrm{Re}}{(\mathrm{Re})}{ }_{\mathrm{A}_{7}}$ & $\frac{\mathrm{Fr}}{(\mathrm{Fr})} \mathrm{A}_{7}$ & $\frac{\mathrm{Eu}}{(\mathrm{Eu})_{\mathrm{A}_{7}}}$ & $\frac{W e}{(W e)}{ }_{A_{7}}$ & $\frac{\rho_{G^{\prime}} \rho_{L}}{\rho_{G^{\prime}} \rho_{L}{ }^{\prime} A_{7}}$ & $\frac{\mathrm{g}}{\mathrm{g}_{0}}$ & \multicolumn{2}{|c|}{$\begin{array}{l}\text { NUMBER OF } \\
\text { COND DATA }\end{array}$} \\
\hline $\mathrm{A}_{7}$ & 1.00 & 1.00 & 1.00 & 1.00 & 1.00 & 1.00 & 113 & 18 & 107 \\
\hline$A_{8}$ & 1.00 & 1.00 & 1.00 & 0.53 & 1.00 & 1.00 & 113 & 5 & 20 \\
\hline$A_{9}$ & 1.00 & 1.00 & 1.00 & 0.14 & 1.00 & 1.00 & 113 & 2 & 6 \\
\hline$A_{10}$ & 1.00 & 1.00 & 1.00 & 0.97 & 1.00 & 1.82 & 113 & 5 & 9 \\
\hline$A_{11}$ & 1.00 & 1.00 & 1.00 & 0.97 & 1.00 & $\underline{7.24}$ & 113 & 7 & 34 \\
\hline$A_{12}$ & 1.00 & 2.00 & 1.00 & 1.12 & 1.09 & 1.00 & 113 & 18 & 35 \\
\hline$A_{13}$ & 1.00 & 1.00 & 1.77 & 1.00 & 1.00 & 1.00 & 65 & 5 & 6 \\
\hline$A_{14}$ & 1.00 & 1.00 & 0.46 & 1.00 & 1.00 & 1.00 & 245 & 17 & 25 \\
\hline
\end{tabular}

Table I-6: Relative velocity and relative dimensionless numbers with respect to the series $A_{7}$ for eight test series of the rotating $5 \mathrm{~mm}$ test section.

\begin{tabular}{|c|c|c|c|c|c|c|c|c|c|}
\hline $\begin{array}{l}\text { TEST } \\
\text { SERIES }\end{array}$ & $\begin{array}{c}\rho_{L} \\
\mathrm{~kg} / \mathrm{m}^{3}\end{array}$ & $\begin{array}{c}\mu_{L} \\
\mathrm{~Pa} . \mathrm{s} \\
10^{-3}\end{array}$ & $\begin{array}{c}\sigma \\
\mathrm{N} / \mathrm{m} \\
10^{-3}\end{array}$ & $\begin{array}{c}{ }^{\rho_{\mathrm{G}}} \\
\mathrm{kg} / \mathrm{m}^{3}\end{array}$ & $\begin{array}{c}\mu_{G} \\
P a . s \\
10^{-6}\end{array}$ & $\begin{array}{c}\mathrm{p} \\
\mathrm{Pa} \\
10^{6}\end{array}$ & $\begin{array}{l}\mathrm{T} \\
{ }^{\circ} \mathrm{C}\end{array}$ & $\frac{\mathrm{g}}{\mathrm{g}_{0}}$ & FLUIDA \\
\hline $\mathrm{A}_{7}$ & 998 & 1.00 & 72.8 & 4.76 & 19.6 & 2.95 & 20 & 113 & water $/ \mathrm{He}$ \\
\hline$A_{8}$ & 998 & 1.00 & 72.8 & 4.76 & 19.4 & 1.55 & 20 & 113 & water $/ 14 \%$ air \\
\hline $\mathrm{A}_{9}$ & 998 & 1.00 & 72.8 & 4.76 & 18.4 & 0.40 & 20 & 113 & water/air \\
\hline$A_{10}$ & 998 & 1.00 & 72.8 & 8.66 & 19.4 & $\overline{2.85}$ & 20 & 113 & water $/ \mathrm{He}$ \\
\hline$A_{11}$ & 998 & 1.00 & 72.8 & $\underline{34.4}$ & 18.9 & 2.87 & 20 & 113 & water/air \\
\hline$A_{12}$ & 986 & 0.50 & 67.1 & 4.70 & 21.0 & 3.26 & 55 & 113 & water $/ \mathrm{He}$ \\
\hline$A_{13}$ & 998 & 1.00 & 72.8 & 4.76 & 19.6 & 2.95 & 20 & $\underline{65}$ & water $/ \mathrm{He}$ \\
\hline$A_{14}$ & 998 & 1.00 & 72.8 & 4.76 & 19.6 & 2.95 & 20 & 245 & water $/ \mathrm{He}$ \\
\hline
\end{tabular}

Table I-7: Fluid properties and system parameters for the eight test series of the rotating $5 \mathrm{~mm}$ test section. 


\begin{tabular}{|c|c|c|c|c|c|c|c|c|c|}
\hline \multirow[t]{2}{*}{ COND } & \multicolumn{3}{|c|}{ FRICTION MULTIPLIER } & \multicolumn{3}{|c|}{ VOID FRACTION } & \multicolumn{3}{|c|}{ FLOW PATTERN } \\
\hline & $\mathrm{A}_{7}$ & $A_{12}$ & $A_{14}$ & ${ }^{\mathrm{A}} 7$ & ${ }^{A_{12}}$ & $A_{14}$ & $\mathrm{~A}_{7}$ & $A_{12}$ & $A_{14}$ \\
\hline$a_{11}$ & 0.45 & 0.35 & - & 0.28 & 0.29 & - & SS & SS & -- \\
\hline$a_{12}$ & 3.72 & 4.6 & - & 0.50 & 0.54 & 0.44 & SS & SS & SS \\
\hline$a_{14}$ & 8.7 & 9.3 & 5.9 & 0.71 & 0.71 & 0.65 & SW & SW & SS \\
\hline$a_{15}$ & 38.4 & 42.5 & 31.8 & 0.84 & 0.85 & 0.84 & SA & SA & SA \\
\hline$a_{21}$ & 0.90 & 1.35 & 0.04 & 0.24 & 0.25 & 0.23 & ss & SS & SS \\
\hline$a_{22}$ & 2.42 & 2.41 & 1.65 & 0.53 & 0.53 & 0.43 & PL & PL & PL \\
\hline$a_{23}$ & 3.78 & 3.93 & 3.44 & 0.60 & 0.60 & 0.60 & PS & SL & PL \\
\hline$a_{24}$ & 5.3 & 5.5 & 4.5 & 0.65 & 0.65 & 0.64 & SL & SL & SL \\
\hline$a_{25}$ & 17.9 & 19.6 & 14.5 & 0.79 & 0.79 & 0.78 & SA & $S A$ & $\mathrm{SA}$ \\
\hline 31 & 1.16 & 1.39 & 0.93 & 0.22 & 0.21 & 0.20 & PL & PL & PL \\
\hline$a_{32}$ & 2.49 & 2.46 & 1.88 & 0.48 & 0.48 & 0.44 & PL & PL & PL \\
\hline$a_{33}$ & 3.47 & 3.50 & 3.40 & 0.57 & 0.57 & 0.58 & PS & PS & PL \\
\hline$a_{34}$ & 4.4 & 4.6 & 4.0 & 0.62 & 0.63 & 0.62 & SL & $\mathrm{SL}$ & SL \\
\hline$a_{35}$ & 14.0 & 15.2 & 10.8 & 0.78 & 0.76 & 0.75 & SA & SL & SL \\
\hline$a_{41}$ & 1.23 & 1.23 & 1.11 & 0.10 & 0.09 & 0.10 & PL & PL & PL \\
\hline$a_{42}$ & 1.65 & 1.71 & 1.53 & 0.28 & 0.26 & 0.29 & PL & PL & PL \\
\hline$a_{43}$ & 2.25 & 2.33 & 2.11 & 0.41 & 0.40 & 0.43 & PL & PL & PL \\
\hline$a_{44}$ & 2.98 & 3.00 & 2.64 & 0.49 & 0.48 & 0.50 & PS & PS & PS \\
\hline
\end{tabular}

\begin{tabular}{|l|cccccc|}
\hline COND & \multicolumn{7}{|c|}{ FRICTION MULTIPLIER } \\
& $A_{7}$ & $A_{8}$ & $A_{9}$ & $A_{10}$ & $A_{11}$ & $A_{13}$ \\
\hline$a_{22}$ & 2.42 & 2.36 & - & 2.76 & 3.01 & 3.23 \\
$a_{24}$ & 5.3 & 5.5 & 5.7 & 6.5 & 9.1 & 6.3 \\
$a_{25}$ & 17.9 & 18.2 & 18.6 & 23.3 & 29.4 & - \\
\hline$a_{41}$ & 1.23 & - & - & - & 1.30 & 1.28 \\
$a_{42}$ & 1.65 & 1.64 & - & 1.68 & 1.63 & 1.70 \\
$a_{43}$ & 2.25 & 2.30 & - & 2.29 & 2.42 & 2.52 \\
$a_{44}$ & 2.98 & - & - & - & 3.08 & - \\
\hline
\end{tabular}

Table I-8: Results rotat. $5 \mathrm{~mm}$ section. $\mathrm{SW}=$ Strat. Smooth SW = Srat. Wavy PS = Plug or Slug $\mathrm{SS}=$ Plug or Slug $\mathrm{SA}=$ Semi Annular $a_{11}-a_{15}: \nabla$ or $v$ $a_{21}-a_{25}:$ or $a_{31}-a_{35}: 0$ or $a_{41}-a_{44}: \Delta$ or $\Delta$ 


\section{APPENDIX II}

TEST CONDITIONS AND EXPERIMENTAL RESULTS OF THE SCALING TEST B

The scaling test $B$ includes measurements of the stationary $100 \mathrm{~mm}$ test section, the stationary $50 \mathrm{~mm}$ test section and the rotating $5 \mathrm{~mm}$ test section.

The superficial velocities, single-phase liquid pressure drop and dimensionless numbers for the $100 \mathrm{~mm}$ test section (test series $B_{1}$ ) are listed in table II-1. A test condition is denoted by $b_{* *}$, where the first subscript indicates the adjusted liquid velocity and the second one the gas velocity. The higher the number the higher the velocity.

The relative velocity and the relative dimensionless numbers (with respect to the test series $B_{1}$ ) of the $50 \mathrm{~mm}$ and $5 \mathrm{~mm}$ test section are listed in table II-2.

Fluid properties and system parameters can be found in Table II-3. The experimental results of the friction multiplier $\phi_{L}^{2}$, the void fraction and the flow pattern are given in table II-4. 


\begin{tabular}{|c|c|c|c|c|c|c|c|}
\hline $\begin{array}{l}\text { TEST } \\
\text { COND }\end{array}$ & $U_{L}$ & $\mathrm{U}_{\mathrm{G}}$ & {$\left[\frac{\Delta \mathrm{P}}{\Delta \mathrm{L}}\right]_{\mathrm{L}}$} & Re & $\mathrm{Fr}$ & $\mathrm{Eu}$ & We \\
\hline & $\mathrm{m} / \mathrm{s}$ & $\mathrm{m} / \mathrm{s}$ & $\mathrm{Pa} / \mathrm{m}$ & -- & -- & - & -- \\
\hline$b_{13}$ & 0.204 & 2.89 & 6.9 & 6600 & 0.040 & 3150 & 139 \\
\hline$b_{14}$ & 0.201 & 6.07 & 6.7 & 6500 & 0.039 & 3240 & 135 \\
\hline$b_{23}$ & 0.238 & 3.23 & 9.0 & 7700 & 0.055 & 2310 & 189 \\
\hline$b_{24}$ & 0.238 & 6.25 & 9.0 & 7700 & 0.055 & 2310 & 189 \\
\hline$b_{33}$ & 0.357 & 2.86 & 18.3 & 11500 & 0.123 & 1030 & 426 \\
\hline $\mathrm{b}_{34}$ & 0.361 & 6.20 & 18.7 & 11700 & 0.126 & 1010 & 436 \\
\hline$b_{41}$ & 0.72 & 0.62 & 62.1 & 23300 & 0.50 & 253 & 1728 \\
\hline$b_{42}$ & 0.70 & 1.13 & 58.5 & 22500 & 0.47 & 271 & 1615 \\
\hline$b_{43}$ & 0.67 & 3.09 & 55.0 & 21700 & 0.44 & 291 & 1505 \\
\hline$b_{44}$ & 0.62 & 6.34 & 48.0 & 20000 & 0.37 & 341 & 1285 \\
\hline$b_{51}$ & 0.89 & 0.59 & 91 & 28800 & 0.77 & 165 & 2660 \\
\hline$b_{52}$ & 0.94 & 1.06 & 100 & 30500 & 0.86 & 147 & 2970 \\
\hline$b_{53}$ & 0.93 & 2.86 & 98 & 30100 & 0.84 & 151 & 2890 \\
\hline$b_{54}$ & 0.96 & 6.12 & 102 & 30900 & 0.88 & 143 & 3060 \\
\hline$b_{61}$ & 1.56 & 0.59 & 242 & 50400 & 2.35 & 54 & 8140 \\
\hline$b_{62}$ & 1.54 & 1.02 & 235 & 49600 & 2.28 & 56 & 7880 \\
\hline$b_{63}$ & 1.60 & 3.05 & 251 & 51600 & 2.46 & 51 & 8500 \\
\hline$b_{64}$ & 1.43 & 5.99 & 208 & 46300 & 1.98 & 64 & 6860 \\
\hline
\end{tabular}

Table II-1: Superficial liquid velocity, superficial gas velocity, single-phase liquid pressure drop and values of the dimensionless groups for the test conditions of series $B_{1}$ (stationary $100 \mathrm{~mm}$ test section). 


\begin{tabular}{|c|c|c|c|c|c|c|c|c|c|}
\hline $\begin{array}{l}\text { TEST } \\
\text { SERIES }\end{array}$ & $\frac{\mathrm{U}}{(\mathrm{U})}{ }_{\mathrm{B}_{1}}$ & $\frac{\mathrm{Re}}{(\operatorname{Re})_{B_{1}}}$ & $\frac{F r}{(F r)_{B_{1}}}$ & $\frac{E u}{(E u)_{B_{1}}}$ & $\frac{W e}{(W e)_{B_{1}}}$ & $\frac{\rho_{G} / \rho_{L}}{\rho_{G^{\prime}} \rho_{L}{ }^{\prime}}$ & DECL & \multicolumn{2}{|c|}{$\begin{array}{l}\text { NUMBER OF } \\
\text { COND DATA }\end{array}$} \\
\hline $\mathrm{B}_{1}$ & 1.00 & 1.00 & 1.00 & 1.00 & 1.00 & 1.00 & horiz & 18 & 18 \\
\hline $\mathrm{B}_{2}$ & 0.68 & 1.00 & 1.00 & 2.01 & 0.10 & 1.00 & horiz & 17 & 34 \\
\hline $\mathrm{B}_{3}$ & 0.68 & 1.00 & 1.00 & 1.65 & 0.10 & 0.82 & $1: 245$ & 18 & 72 \\
\hline$B_{4}$ & 3.37 & 1.00 & 1.00 & 0.67 & 0.23 & 1.00 & $\frac{\mathrm{g}}{\mathrm{B}_{0}}=245$ & 18 & 31 \\
\hline
\end{tabular}

Table II-2: Relative velocity and relative dimensionless numbers of the test series $B_{2}(50 \mathrm{~mm}), B_{3}(50 \mathrm{~mm})$, and $B_{4}(5 \mathrm{~mm})$ with respect to the test series $B_{1}(100 \mathrm{~mm})$.

\begin{tabular}{|c|c|c|c|c|c|c|c|c|c|}
\hline $\begin{array}{l}\text { TEST } \\
\text { SERIES }\end{array}$ & $\begin{array}{c}P_{L} \\
\mathrm{~kg} / \mathrm{m}^{3}\end{array}$ & $\begin{array}{c}\mu_{L} \\
\mathrm{~Pa} \cdot \mathrm{s} \\
10^{-3}\end{array}$ & $\begin{array}{c}\sigma \\
N / m \\
10^{-3}\end{array}$ & $\begin{array}{c}{ }^{\rho} \mathrm{G} \\
\mathrm{kg} / \mathrm{m}^{3}\end{array}$ & $\begin{array}{c}{ }_{\mathrm{G}} \\
\mathrm{Pa} . \mathrm{s} \\
10^{-6}\end{array}$ & $\begin{array}{c}\mathrm{p} \\
\mathrm{Pa} \\
10^{6}\end{array}$ & $\begin{array}{l}\mathrm{T} \\
{ }^{\circ} \mathrm{C}\end{array}$ & $\begin{array}{c}\text { DIAM } \\
10^{-3}\end{array}$ & FLUIDA \\
\hline $\mathrm{B}_{1}$ & 840 & 2.74 & 26.5 & 1.24 & 18.9 & 0.110 & 37 & 105.5 & gasoil/air \\
\hline $\mathrm{B}_{2}$ & 999 & 1.05 & 73.0 & 1.47 & 18.2 & 0.123 & 18 & 49.8 & water/air \\
\hline $\mathrm{B}_{3}$ & 999 & 1.05 & 73.0 & 1.21 & 18.2 & 0.101 & 18 & 49.8 & water/air \\
\hline$B_{4}$ & 986 & 0.50 & 67.1 & 1.45 & 21.0 & 0.990 & 55 & 4.9 & water/He \\
\hline
\end{tabular}

Table II-3: Fluid properties and system parameters for the test series of the scaling test $B$. 


\begin{tabular}{|c|c|c|c|c|c|c|c|c|c|c|c|c|}
\hline \multirow[t]{2}{*}{ COND } & \multicolumn{4}{|c|}{ FRICTION MULTIPLIER } & \multicolumn{4}{|c|}{ VOID FRACTION } & \multicolumn{4}{|c|}{ FLOW PATTERN } \\
\hline & $B_{1}$ & $\mathrm{~B}_{2}$ & $\mathrm{~B}_{3}$ & $B_{4}$ & $B_{1}$ & $B_{2}$ & $\mathrm{~B}_{3}$ & $\mathrm{~B}_{4}$ & $\mathrm{~B}_{1}$ & $\mathrm{~B}_{2}$ & $\mathrm{~B}_{3}$ & $\mathrm{~B}_{4}$ \\
\hline$b_{13}$ & 4.4 & 12.0 & 4.6 & 4.5 & 0.61 & 0.72 & 0.64 & 0.60 & SL & PL & SS & SL \\
\hline$b_{14}$ & 9.9 & 12.1 & $15 . ?$ & 9.3 & 0.70 & 0,74 & 0.75 & 0.71 & SL & $\mathrm{SL}$ & SL & SL \\
\hline 23 & 6.4 & 8.4 & 4.2 & 5.3 & 0.72 & 0.72 & 0.62 & 0.61 & SL & PL & ss & SL \\
\hline $\mathrm{b}_{24}$ & 9.4 & 10.8 & 11.0 & 10.7 & 0.73 & 0.73 & 0.76 & 0.69 & SL & SL & SL & SL \\
\hline $\mathrm{b}_{33}$ & 3.8 & 5.2 & 5.6 & 6.2 & 0.70 & 0.69 & 0.60 & 0.65 & SL & PL & SL & PL \\
\hline $\mathrm{b}_{34}$ & 10.2 & 8.2 & 7.9 & 8.2 & 0.76 & 0.70 & 0.72 & 0.67 & SL & SL & SL & SL \\
\hline$b_{41}$ & 1.38 & 1.47 & 1.61 & 1.31 & 0.31 & 0.34 & 0.33 & 0.30 & PL & PL & PL & PL \\
\hline$b_{42}$ & 1.76 & - & 2.32 & 1.84 & 0.50 & - & 0.45 & 0.39 & SL & -- & PL & PL \\
\hline$b_{43}$ & 3.3 & 4.6 & 4.6 & 4.8 & 0.65 & 0.61 & 0.62 & 0.58 & SL & PL & PL & PL \\
\hline $\mathrm{b}_{44}$ & 5.3 & 6.2 & 6.0 & 7.0 & 0.77 & 0.66 & 0.67 & 0.62 & SL & SL & $\mathrm{SL}$ & SL \\
\hline$b_{51}$ & 1.27 & 1.45 & 1.50 & 1.33 & 0.32 & 0.30 & 0.30 & 0.27 & PL & PL & PL & PL \\
\hline$b_{52}$ & 1.47 & 1.97 & 2.01 & 1.91 & 0.47 & 0.40 & 0.35 & 0.36 & PL & PL & PL & PL \\
\hline $\mathrm{b}_{53}$ & 2.94 & 3.50 & 3.63 & 3.58 & 0.56 & 0.57 & 0.57 & 0.55 & SL & PL & PL & PL \\
\hline$b_{54}$ & 4.8 & 5.2 & 5.3 & 6.6 & 0.74 & 0.63 & 0.64 & 0.65 & SL & SL & SL & SL \\
\hline $\mathrm{b}_{61}$ & 1.23 & 1.29 & 1.36 & 1.32 & 0.24 & 0.22 & 0.22 & 0.20 & PL & PL & PL & PL \\
\hline$b_{62}$ & 1.32 & 1.62 & 1.67 & 1.66 & 0.34 & 0.31 & 0.30 & 0.28 & PL & PL & PL & PL \\
\hline$b_{63}$ & 2.09 & 2.84 & 2.87 & 2.91 & 0.56 & 0.49 & 0.49 & 0.48 & SL & PL & PL & PL \\
\hline$b_{64}$ & 3.2 & 4.3 & 4.3 & 5.5 & 0.69 & 0.58 & 0.59 & 0.60 & SL & SL & SL & SL \\
\hline
\end{tabular}

Table II-4: Experimental results for the scaling test $B$.

Flow Pattern:SS = Stratified Smooth

$$
\begin{aligned}
& \text { PL }=\text { Plug } \\
& \text { SL }=\text { Slug }
\end{aligned}
$$

$B_{1}: 100 \mathrm{~mm}$ test section

$B_{2}: 50 \mathrm{~mm}$ test section

$B_{3}: 50 \mathrm{~mm}$ test section

$B_{4}: 5 \mathrm{~mm}$ test section 


\section{CAPACITANCE MEASUREMENTS WITH CONDUCTING MEDIA}

A mixture of a conducting and a non conducting medium, such as a two-phase flow of air and water, is an inhomogeneous mixture whose relative permittivity is dependent on the frequency. This dispersion is known as the Maxwell-Wagner effect (Hasted, 1973), and the consequences for the measurements with the sensors used in this investigation are given below.

Consider first a parallel-plate capacitor of gap d, filled with a homogeneous material of relative permittivity $\varepsilon_{r}$ (assumed frequency independent) and conductivity $\kappa$. When the potential difference between the plates is $V$, the charge per unit area is:

$$
Q=\frac{\varepsilon_{r} \varepsilon_{0} V}{d}
$$

and the current density is:

$$
I=\frac{d Q}{d t}+\kappa \frac{V}{d}
$$

If $V$ is an alternating potential, $V=V_{0} e^{j \omega t}$, the current density becomes:

$$
I=\left(j \omega \varepsilon_{r} \varepsilon_{0}+k\right) \frac{V}{d}=j \omega \varepsilon_{0}\left(\varepsilon_{r}-\frac{j k}{\omega \varepsilon_{0}}\right) \frac{V}{d}
$$

From these equations the medium can be considered as having a complex conductivity, $k+j \omega \varepsilon_{r} \varepsilon_{0}$, or a complex relative permittivity $\varepsilon_{r}-j \kappa / \omega \varepsilon_{0}$. With the latter view the dielectric loss $\varepsilon^{\prime \prime}=\kappa / \omega \varepsilon_{0}$ is inversely proportional to the frequency, becoming zero at high frequencies.

Distilled water in equilibrium with atmospheric carbon dioxide has a conductivity $k=10^{-4} 1 / \Omega \mathrm{m}$. At $1 \mathrm{MHz}$ the dielectric $10 \mathrm{ss}$ is small, about $2 \%$ of the relative permittivity. At higher values of $k$ or lower frequencles the dielectric loss becomes considerable. However if a phase-sensitive detector is used the capacitance $C$ and the conductance $K$ can be measured seperately and hence the conductive part doesn't have to be necessarily smal1. The meter (frequency $=1 \mathrm{MHz}$ ) used here measures the capacitance correct if $\omega C / K>1(\omega C / K$ is called the quality of the meter). 


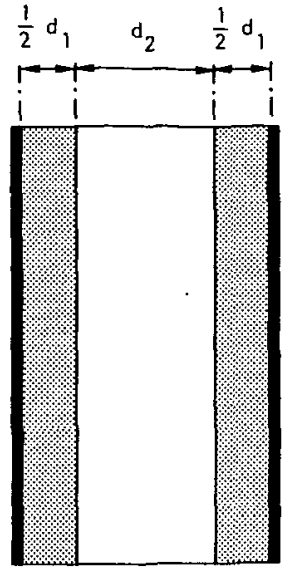

Figure III-1:

Parallel-plate capacitor.
For the 5 and $50 \mathrm{~mm}$ sensor with electrodes flush with the wall this requirement is satisfied as long as $k<50 \times 10^{-4} 1 / \Omega \mathrm{m}$. If the dielectric speciment is not homogeneous but contains regions of different permittivity, such as the vold sensor with an acrylate tube in which water or an air-water mixture flows, the permittivity of the medium appears to be frequency dependent. The origin of this effect is shown by means of a simple model of the sensor, that of parallel slabs of dielectric material of total thickness $d_{1}+d_{2}$, see figure III-1. The first material having permittivity $\varepsilon_{1}$ and zero conductivity, the second permittivity $\varepsilon_{2}$ and conductivity $x$.

This configuration is equivalent to two capacitors $C_{1}$ and $C_{2}$ in series, the capacitance per unit area being:

$$
c_{1}=\varepsilon_{1} \frac{\varepsilon_{0}}{d_{1}} \quad \text { and } \quad C_{2}=\left(\varepsilon_{2}-\frac{j k}{\varepsilon_{0} \omega}\right) \frac{\varepsilon_{0}}{d_{2}}
$$

and the total capacitance per unit area:

$$
C_{T}=\frac{C_{1} C_{2}}{C_{1}+C_{2}}=\frac{\varepsilon_{0}\left(\left(\varepsilon_{2}-j k / \varepsilon_{0} \omega\right) \varepsilon_{1} / d_{1} d_{2}\right)}{\left(\varepsilon_{2}-j k / \varepsilon_{0} \omega\right) / d_{2}+\varepsilon_{1} / d_{1}}
$$

The complex relative permittivity is:

$$
\varepsilon_{T}^{*}=\frac{\varepsilon_{1} \varepsilon_{2}\left(\varepsilon_{1} d_{2}+\varepsilon_{2} d_{1}\right)+\left(k / \varepsilon_{0} \omega\right)^{2} \varepsilon_{1} d_{i}-j\left(k / \varepsilon_{0} \omega\right)\left(\varepsilon_{1} d_{2}+\varepsilon_{1} d_{2}^{*}\right)}{\left(\varepsilon_{1} d_{2}+\varepsilon_{2} d_{j}\right)^{2}+\left(k d_{i} / \varepsilon_{0} \omega\right)^{2}}
$$

where $d_{i}=\frac{d_{1}}{d_{1}+d_{2}}$ and $d_{2}^{\prime}=\frac{d_{2}}{d_{1}+d_{2}}$ 
With a phase-sensitive detector only the real part is measured:

$$
\varepsilon_{T}=\frac{\varepsilon_{1} \varepsilon_{2}\left(\varepsilon_{1} d_{2}^{\prime}+\varepsilon_{2} d_{1}^{\prime}\right)+\left(\kappa / \varepsilon_{0} \omega\right)^{2} \varepsilon_{1} d_{1}}{\left(\varepsilon_{1} d_{2}^{\prime}+\varepsilon_{2} d_{1}\right)^{2}+\left(k d_{1} / \varepsilon_{0} \omega\right)^{2}}
$$

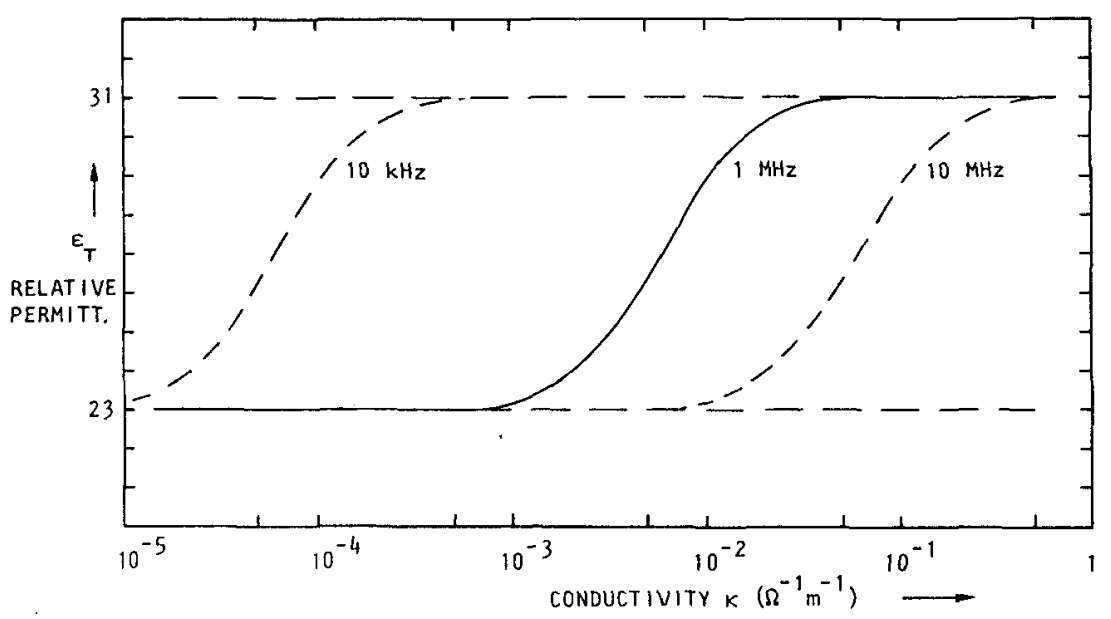

Figure III-2: The relative permittivity $\varepsilon_{T}$ as function of the conductivity and angular frequency.

The relative permittivity $\varepsilon_{T}$ of the configuration is both dependent on the frequency and the conductivity. In figure III-2 the permittivity is given as a function of the conductivity for a sensor with an acrylate inner tube of thickness 0.055 times the inner diameter and filled with water: $\varepsilon_{1}=3.1, \varepsilon_{2}=80, d_{j}=0.1$ and $\dot{d}_{2}=0.9$.

At high frequencies or zero conductivity $\varepsilon_{\mathrm{T}}=23$ and at zero frequency or high conductivities $\varepsilon_{T}=31$.

Due to the Maxwell-wagner effect at a conductivity of $50 \times 10^{-4} 1 / \Omega \mathrm{m}$ and frequency of $1 \mathrm{MHz}$ the permittivity increases $13 \%$ with respect to the value at $10^{-4} 1 / \Omega \mathrm{m}$. 


\begin{tabular}{|c|c|c|}
\hline $\mathrm{C}$ & capacitance & {$[F]$} \\
\hline$c^{\prime}$ & capacitance per unit length & {$[F / m]$} \\
\hline$\Delta C$ & capacitance difference & {$[F]$} \\
\hline$D$ & tube diameter & {$[\mathrm{m}]$} \\
\hline $\mathbf{E}$ & electric field & {$[\mathrm{V} / \mathrm{m}]$} \\
\hline $\mathbf{f}$ & plus or slug frequency & {$[1 / 9]$} \\
\hline 8 & acceleration of the gravity & {$\left[\mathrm{m} / \mathrm{s}^{2}\right]$} \\
\hline $\mathbf{s}_{0}$ & acceleration of the natural gravity & {$\left[\mathrm{m} / \mathrm{s}^{2}\right]$} \\
\hline$\underline{k}$ & unit vector in the direction of the gravity & {$[-]$} \\
\hline $\mathrm{K}$ & electric conductance & {$[1 / \Omega]$} \\
\hline $\mathrm{K}$ & flow coefficient of integral arifices & {$[-]$} \\
\hline $\mathrm{L}$ & characteristic length of the flow & {$[\mathrm{m}]$} \\
\hline $\mathrm{L}$ & axial length of electrode & [m] \\
\hline$\Delta \mathrm{L}$ & distance between pressure taps & {$[\mathrm{m}]$} \\
\hline M & molecular weight & $\cdot[\mathrm{kg} / \mathrm{mol}]$ \\
\hline $\mathrm{p}$ & pressure & {$[\mathrm{Pa}]$} \\
\hline$p$ & pitch parameter & {$[-]$} \\
\hline$\Delta \mathrm{p}$ & pressure loss between pressure taps & {$[\mathrm{Pa}]$} \\
\hline$\Delta \mathrm{P}_{\text {or }}$ & pressure loss over orifice & {$[\mathrm{Pa}]$} \\
\hline Q & electric charge on electrode & {$[A . S]$} \\
\hline$r$ & co-ordinate in radial direction & {$[\mathrm{m}]$} \\
\hline$\underline{r}$ & position vector & {$[\mathrm{m}]$} \\
\hline $\mathbf{R}$ & tube radius & {$[\mathrm{m}]$} \\
\hline $\mathbf{R}$ & radius of the rotor & {$[\mathrm{m}]$} \\
\hline $\mathrm{R}$ & gas constant & {$[\mathrm{J} / \mathrm{mol} \cdot \mathrm{K}]$} \\
\hline$R_{1} R_{2}$ & radius of the curvature of a free surface & {$[\mathrm{m}]$} \\
\hline $\mathbf{s}$ & pitch of the helix & {$[\mathrm{m}]$} \\
\hline$t$ & time & {$[s]$} \\
\hline $\mathrm{T}$ & temperature & {$[\mathrm{K}]$} \\
\hline$\underline{\mathbf{u}}$ & velocity vector & {$[\mathrm{m} / \mathrm{s}]$} \\
\hline $\mathrm{u}$ & characteristic velocity & {$[\mathrm{m} / \mathrm{s}]$} \\
\hline $\mathrm{U}_{\mathrm{L}}$ & superficial liquid velocity & {$[\mathrm{m} / \mathrm{s}]$} \\
\hline $\mathrm{U}_{\mathrm{G}}$ & superficial gas velocity & {$[\mathrm{m} / \mathrm{s}]$} \\
\hline
\end{tabular}




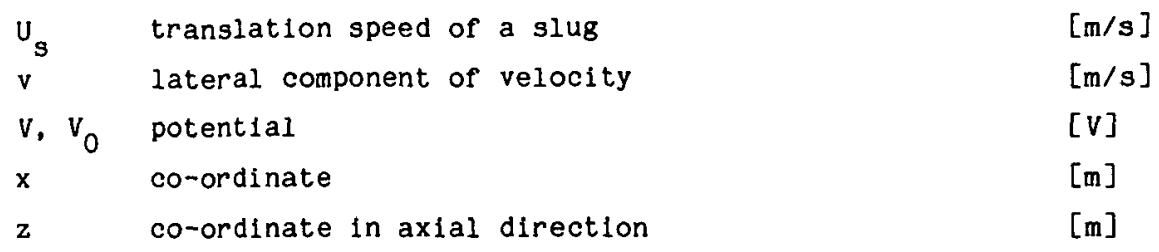

$\begin{array}{lll}\alpha & \text { vold fraction } & {[-]} \\ \varepsilon_{0} & \text { permittivity of free space } & {[\mathrm{F} / \mathrm{m}]} \\ \varepsilon_{r} & \text { relative permittivity } & {[-]} \\ \varepsilon^{\prime \prime} & \text { dielectric loss } & {[-]} \\ \phi & \text { co-ordinate in circumferential direction } & {[-]} \\ \kappa & \text { conductivity } & {[1 / \Omega \mathrm{m}]} \\ \lambda & \text { input volume fraction liquid } & {[-]} \\ \mu & \text { dynamic viscosity } & {[\mathrm{Pa} . \mathrm{s}]} \\ \xi & \text { dimensionless co-ordinate } & {[-]} \\ \rho & \text { density } & {\left[\mathrm{kg} / \mathrm{m}^{3}\right]} \\ \sigma & \text { surface tension } & {[\mathrm{N} / \mathrm{m}]} \\ \theta & \text { angle between the flow and the gravity } & {[-]} \\ \omega & \text { angular velocity } & {[1 / \mathrm{s}]}\end{array}$

subscripts

$\begin{array}{llll}1 & \text { original flow } & \text { a } & \text { acceleration } \\ 2 & \text { model flow } & \text { e } & \text { elevation } \\ \text { L liquid } & \text { gas } & \text { f } & \text { frictional } \\ \text { G characteristic point } & \mathrm{m} & \text { measured } \\ \text { char } & \text { m } & \text { mixture } \\ & & \text { t } & \text { total }\end{array}$

superscripts

dimensionless quantity 
$E=\frac{{ }^{p_{G}}}{\rho_{G} U_{L}^{2}}$

$E u=\frac{p_{G}}{\rho_{L} U_{L}^{2}}$

$F r=\frac{U_{L}^{2}}{g L}$

$Q=\frac{\rho_{L} \sigma^{3}}{8 \mu_{L}^{4}}$

$R e=\frac{\rho_{L} U_{L} L}{\mu_{L}}$

$W e=\frac{\rho_{L} U_{L}^{2} L}{\sigma}$

$\phi_{L}^{2}=\frac{(\Delta p / \Delta L)_{f}}{(\Delta p / \Delta L)_{L}}$

$x^{2}=\frac{(\Delta p / \Delta L)_{L}}{(\Delta p / \Delta L)_{G}}$
Euler number

alternative Euler number.

Froude number

liquid-property or Morton number

Reynolds number

Weber number

friction multiplier

Lockhart-Martinelli parameter 


\section{LIST OF REFERENCES}

Abouelwafa M.S.A. and Kendall E.J.M. Optimization of continuous wave nuclear magnetic resonance to determine in situ volume fractions and individual flow rates in two component mixtures. Rev. Sci. Instrum., Vol. 50, no 12, pp 1545-1549, 1979.

Abouelwafa M.S.A. and Kendall E.J.M.

The use of capacitance sensors for phase percentage determination in multiphase pipelines.

I.E.E.E. Transactions on instrumentation and measurement, Vol. 29, no 1 , pp 24-27, 1980 .

Akagawa K., Hamaguchi H., Sakaguchi T. and Ikari T.

Studies on the fluctuations of pressure drop in two-phase slug flow.

Bull. of the J.S.M.E., Vol. 14, no 71 , pp $447-469,1971$.

Baker 0 .

Simultaneous flow of oil and gas.

Oil and gas J., Vol. 53, pp 185-192, 1954.

Barnea D., Luninski $Y$. and Taitel $Y$.

Flow pattern in horizontal and vertical two-phase flow in small diameter pipes.

Can. J. of Chem. Eng., vol. 61, pp 617-620, 1983

Barnea D., Shoham O., Taitel Y. and Dukler A.E.

Flow pattern transition for gas-liquid flow in horizontal and inclined pipes.

Int. J. Multiphase flow, Vol. 6, no 3, pp 217-225, 1980.

Baroczy C. J.

A systematic correlation for two-phase pressure drop. Chem. Eng. Prog. Symp. Ser., vol. 62, pp 232-249,1965.

Beattie H. and Whalley P.B.

A simple two-phase frictional pressure drop calculation method. Int. J. Multiphase flow, Vol. 8, no 1, pp 83-87, 1982.

Bell K.J., Taborek J. and Fenoglio F.

Interpretation of horizontal in-tube condensation heat transfer correlations with a two-phase flow regime map.

Chem. Eng. Progr. Symp. Ser., Vol 66, pp 150-163, 1969.

Biswas J. and Greenfield P. F.

Two-phase flow through vertical cappillaries; existence of a stratified flow pattern.

Int. J. Multiphase Flow, Vol. 11, no. 4, pp 553-563, 1985.

Borst J.C.

Capacitieve sensor voor gas-volumefractie metingen. (In Dutch).

Research Report, Dept. of Mech. Eng.

Delft University of Technology, 1983. 
Borst J.C.

Een capacitieve sensor voor het bepalen van gas-volumefracties in twee-fasen mengseis. (In Dutch).

M. Sc. Thesis, MEAH-Report 51

Delft University of Technology, 1985.

Bruggeman D.A.G.

Berechnung verschiedener physikalischer Konstanten von heterogenen

Substanzen.

Ann. Physik, Vol. 24, pp 636-679, 1935.

Buckingham $\mathrm{E}$.

On physically similar systems.

Physical Review, Vol. 4, no 4; pp 345-376, 1914.

Chesters A.K.

The applicability of dynamic-similarity criteria to isothermal, 1iquid-gas, two-phase flows without mass transfer.

Int. J. Mult 1phase Flow, Vol. 2, pp 191-212, 1975.

Chesters A.K.

A note on the centrifugal scaling of horizontal isothermal, liquid-gas flows without mass transfer.

Int. J. Multiphase Flow, Vol. 3, pp 235-241, 1977.

Chesters A.K.

Fundamental problems in gas-liquid two-phase flow.

Ph. D. Thesis, Delft University of Technology, 1978.

Chrisholm D.

Pressure gradients due to friction during the flow of evaporating two-phase mixtures in smooth tubes and channels.

Int. J. Heat Mass Transfer, Vol. 16, pp 347-358, 1973.

DeGance A.E. and Atherton R.W.

Chemical engineering aspects of two-phase flow.

Chem. Eng., Vol. 77, no 6, pp 95-103, 1970a.

DeGance A.E. and Atherton R.W.

Horizontal-flow correlations.

Chem. Eng., Vol. 77, no 6, pp 135-139, $1970 \mathrm{~b}$.

De La Rue R.E. and Toblas W.

on the conductivity of dispersions.

J. Electrochem. Soc., Vol. 106, pp 827-832, 1959.

Delnaye J.M. and Cognet G.

Measuring techniques in gas-11quid two-phase flows.

Springer-Verlag, Berlin, 1984.

Dorfman L.A.

Hydrodynamic resistance and the heat loss of rotating solids.

Translated by $\mathrm{N}$. Kemmer

O11ver \& Boyd, London, p 175, 1963. 
Dukler A.E., Wicks M. and Cleveland R.G.

Frictional pressure drop in two-phase flow:

A. A comparison of existing correlations for pressure loss and holdup. A.I.Ch.E. J.. Vol. 10, no 1, pp 38-43, 1964 a.

B. An approach through similarity analysis.

A.I.Ch.E. J., Vol. 10, no 1, pp 44-51, 1964 b.

Dijkshoorn A.

Een horizontale opstelling voor twee-fasen stroming. (In Dutch).

Research Report, Dept. of Mech. Eng.

Delft University of Technology, 1985.

Dijkshoorn A.

Het schalen van twee-fasen stroming. (In Dutch).

M. Sc. Thesis, MEAH-Report 64 ,

Delft University of Technology, 1986.

Fricke $\mathrm{H}$. and Morse $\mathrm{S}$.

Phys. Rev., Vol. 25, p 361, 1925.

Friedel L. and Mayinger F.

Scaling of two-phase friction pressure drop.

European two-phase flow meeting, Harwell, 3-7 june 1974.

Friedel L.

Improved friction pressure drop correlation for horizontal and vertical two-phase pipe flow.

European two-phase flow group meeting, paper E2, Ispra Italy, 1979.

Geld C.W.M. van der

On phase distribution transitions in vertical evaporator tubes.

Ph. D. Thesis, Eindhoven Univ. of Technology, 1985.

Govier G. W. and Omer M. M.

Can. J. of Chem. Eng., vol. 40, pp 93-104, 1962.

Gregory G.A. and Scott D.S.

Correlation of liquid slug velocity and frequency in horizontal co-current gas-liquid slug flow.

A.I.Ch.E. J.., Vol. 5, pp 933-935, 1969.

Gregory G.A. and Mattar L.

An in-situ volume fraction sensor for two-phase flow of non-electrolytes. J. of Canadian Petroleum Technology, Vol. 12, pp 48-52, 1973.

Hartog J.P. den

Mechanical vibrations.

McGraw-Hill Book Company, 1947.

Hasted J.B.

Aqueous Dielectrics.

Chapman and Hall, London, 1973. 
Hewitt G.F.

Measurements of two-phase flow parameters.

Academic Press, New York, 1978.

Hewitt G.F.

Pressure drop (chapter 2.2), Vold Fraction (chapter 2.3).

In: "Handbook of multiphase system", Hetsroni G. (Editor).

McGraw-Hill Book Company, 1982.

Hoogendoorn C.J.

Gas-liquid flow in horizontal pipes.

Chem. Eng. Sc., Vol. 9, pp 205-217, 1959.

Jones $0 . C$. and Zuber $\mathrm{N}$.

The interrelation between void fraction fluctuations and flow patterns in two-phase flow.

Int. J. Multiphase Flow, Vol. 2, pp 273-304, 1975.

Kvernvold O., Vindoy V., Sondtvedt T., Saasen A. and Selmer-Olsen S.

Velocity distribution in horizontal slug flow.

Int. J. Multiphase flow, Vol. 10, pp 441-457, 1984.

Lockhart R.W. and Martinelli R.C.

Proposed correlation of data for isothermal two-phase, two-component flow in pipes.

Chem. Eng. Progr., Vol. 45, pp 39-48, 1949.

Mandhane J.M., Gregory G.A. and Aziz K.

A flow pattern map for gas-liquid flow in horizontal pipes.

Int. J. Multiphase Flow, Vol. 1, pp 537-553, 1974.

Mandhane J.M., Gregory G.A. and Aziz K.

Critical evaluation of friction pressure-drop for gas-liquid flow in

horizontal pipes.

J. Petroleum Technology, pp 1348-1358, 1977.

Matsui G.

Identification of flow regimes in vertical gas-liquid two-phase flow using differential pressure fluctuations.

Int. J. Multiphase Flow, Vol. 10, no 6, pp 711-720, 1984.

Maxwell J.C.

A treatise on electricity and magnetism.

Vol. 1. Third edition. Clarendon Press, oxford, 1881.

Mayinger $F$.

Scaling and modelling laws in two-phase flow and boiling heat transfer.

In: "Two-phase flows and heat transfer", Kakaç S. and Mayinger F. (editors). Hemisphere Publishing Corporation, p 129, 1977.

Merilo M., Dechene R.L. and Cichowlas M.W.

Void fraction measurement with a rotating electric field conductance gauge.

J. of Heat transfer, Vol. 99, p 330, 1977. 
Milne-Thomson L.M.

Theoretical Hydrodynamics.

MacMillan, New York, 1960.

\section{Oya T.}

Upward liquid flow in a small tube into which air streams.

Bulletin of the J.S.M.E.. Vol. 14, pp 1320-1339, 1971.

Oya T.

Upward liquid flow in a small tube into which air streams.

Bulletin of the J.S.M.E., Vol. 15, pp 1522-1555, 1972.

Rehman M., Mukerji S.K. and Murti V.G.K.

Effect of a symmetrically placed dielectric tube on the capacitance of a cylindrical cross-capacitor.

PROC. IEE, Vol. 129, Pt. A, no 5, India, july 1982.

Sangani A. and Acrivos A.

The effective conductivity of a periodic array of spheres.

Proc. R. Soc. London A 386, pp 263-275, 1983.

Schlichting $\mathrm{H}$.

Boundary-layer theory.

McGraw-Hill Book Company, 1979.

Scott D.S.

Properties of co-current gas-liquid flow.

In: "Advances in Chemical Engineering", Vol. 4, pp 199-277, Academic Press, New York, 1963.

\section{Shaw R.}

The influence of hole dimensions on static pressure measurements. J. Fluid Mech., Vol. 7, pp 550-564, 1960.

Simpson H.C., Rooney D.H., Gratton E. and Al-Samarral E.

Two-phase flow in large diameter horizontal lines.

European Two-Phase Flow Group Meet ing, Paper H 6, Grenoble, 1977.

Spedding P.L. and Chen J.J.J.

Pressure drop in two-phase gas-liquid flow in inclined pipes.

Int. J. Multiphase Flow, Vol. 8, no 4, pp 407-431, 1982.

Spedding P.L. and Chen J.J.J.

Holdup in two-phase flow.

Int. J. Multiphase flow, Vol. 10; no 3, pp 307-339, 1984.

Taltel $Y$, and Dukler A.E.

A model for predicting flow regime transitions in horizontal and near horizontal gas-liquid flow.

A.I.Ch.E. J., Vol. 22, no 1, pp 47-55, 1976.

Taitel Y. and Dukler A.E.

A model for slug frequency during gas-liquid flow in horizontal and near horizontal pipes.

Int. J. Multiphase flow, Vol. 3, pp 585-596, 1977. 
Taylor G.I.

Stability of a viscous liquid contained between two rotating cylinders. Phil. Trans. Royal Soc. of London 223A, pp 289-343, 1923.

Thomassen B.M.J. and Lans R. v.d.

Bepaling van de schulfspanning aan een roterende cylinder. (In Dutch).

Rapport Moller-Insti tuut Tilburg, 1982.

Tomida T., Yamamoto T., Takebayashi T. and Okazaki T.

Correlation of data on the apparent friction coefficient in upward two-phase flow of air-liquid mixtures.

J. of Chem. Eng. of Japan, Vol. 8, no 2, pp 113-118, 1975.

Vries D.A. de

Het warmtegeleidingsvermogen van de grond. (In Dutch)

Mededeling van de Landbouw Hogeschool te Wageningen, Vol. 52, pp 1-73, 1952.

Wallis G. B.

One dimensional two-phase flow.

McGraw-Hill Book Company, New York, 1969.

Wallis G.B. and Dobson J.E.

The onset of slugging in horizontal stratifled air-water flow.

Int. J. Multiphase flow, Vol. 1, no 1, pp 173-193, 1973.

Weisman J., Duncan D., Gibson J. and Crawford T.

Effects of fluid properties and plpe diameter on two-phase flow patterns in horizontal lines.

Int. J. Multiphase Flow, Vol. 5, pp 437-462, 1979. 


\section{SAMENVATTING}

Het onderzoek beoogt de gelijkvormigheidscriterla voor isotherme gasvloeistof stromingen, die afgeleid zijn van de basisvergelijkingen en randvoorwaaarden voor twee-fasen stromingen, experimenteel te toetsen en tevens het belang van de verschillende beinvloedingsparameters aan te geven. Daartoe is een twee-fasen stroming van lucht en een water-glycerine mengsel in een $50 \mathrm{~mm}$ diameter buls vergeleken met de stroming van water en helium in een $5 \mathrm{~mm}$ diameter buls, die roteert om een parallelle verticale as. Een algemene voorzlening is gebouwd waarmee drukval, gasvolumefractie en stromingspatroon gemeten kunnen worden bij zwaartekrachten tot maximaal 1200 maal de natuurlijke zwaartekracht, in buizen met een diameter tot $10 \mathrm{~mm}$. Het Froude getal, het Weber getal, het Reynolds getal, de gas-vloelstof dlchtheldsverhouding en de gas-vloeistof snelheidsverhouding zijn gelijk in het 50 en $5 \mathrm{~mm}$ systeem. De samendrukbaarheid en viscositeit van het gas zijn nlet volledig geschaald. Veel aandacht is besteed aan de geometrische gelljkvormigheid van de twee test secties: de lengte-diameter verhouding is gelijk, de gas-inlaat geometrie is gelijkvormig en dezelfde uitstroomcondities zijn aangebracht.

Ten behoeve van de schalingsexperimenten is een capacitieve sensor ontwikkeld voor het meten van de geniddelde gasvolumefractie en de bepaling van het.stromingspatroon (uit het tijdsafhankelijke signaal). Omdat hetzelfde type sensor is gebruikt in beide systemen kunnen de twee stromingen gedetailleerd vergeleken worden.

Op een enkele uitzondering na is de overeenkomst tussen de "verschillende" stromingen zeer goed: de verschillen in dimensieloze drukval $z i j n$ kleiner dan 19\%, de gasvolumefractie komt overeen binnen 0.03 en het stromingspatroon is in zeventien van de achtien onderzochte situaties hetzelfde. De frequentie schaalfactor, die bepaald is uit de gemeten plug en slug frequenties komt bijzonder goed overeen met de theoretische waarde. Alleen bij lage gas- en vloeistofdebieten met een stratified stromingspatroon $z i j n$ grote verschillen gemeten die waarschijnlijk veroorzaakt worden door invloeden van specifleke systeem afhankelijke factoren zoals de uitlijning van de test sectie, terugstroming van vloeistof uit de gas-vloelstof scheider en het trillen van de opstelling. 
De dimensleloze groepen zijn zoveel mogelijk afzonderlijk gevarleerd. In de roterende opstelling kan het Froude getal gemakkelifk veranderd worden, terwijl de andere getallen ongewijzigd blijven. Het Froude getal en de gasvloeistof dichtheldsverhouding blijken de meeste invioed te hebben op de stroming. Bij hoge gasdebieten is echter ook een forse invioed van het weber getal gemeten: een twee maal zo hoge waarde geeft een drukvalverlaging van 23\%. De invloed van het Euler getal en de gas-vloeistof dichtheldsverhouding is van minder belang in het onderzochte gebied. De invloed van het Reynolds getal op de gasvolumefractie en het stromingspatroon is gering. De drukval verandert bijna evenredig met de een-fase drukval van de vloeistof.

De metingen zijn vergeleken met bekende correlaties voor drukval, gasvolumefractie en stromingspatroon uit de literatuur. In het algemeen zullen alleen dimensleloze correlaties reële voorspellingen geven. Het homogene "no-slip" model van Dukler (1964b) geeft de beste overeenkomst met de gemeten drukval. De standaard deviatie van $21 \%$ is echter niet verwonderlijk, aangezien de correlatle geen invloeden van speclfieke twee-fasen parameters zoals het Froude getal en het Weber getal toestaat. Voor het voorspellen van het stromingspatroon biedt het model van Taitel en Dukler (1976) de beste perspectieven. Omdat de correlatie dimensieloos is en gebaseerd op fysische modellen, is het toepassingsgebied groter dan dat van andere, vaak niet dimensieloze voorspellings methoden.

Tenslotte $z$ ljn enkele voorbeelden van de schaling van grote diameter leidingen met een twee-fasen stroming van olie en gas onder hoge druk gepresenteerd. B1j een lengte schaalfactor van 1/30 kan gelijkheld van het Froude getal, de gas-vloelstof dichtheldsverhouding en hetzij het Reynolds getal hetzij het weber getal gerealiseerd worden. Samendrukbaarheld en viscositeit van het gas zijn in het algemeen niet volledig geschaald. 


\section{NAWOORD}

Mijn dank gaat uit naar prof.dr.ir. G. Ooms, wiens enthousiaste houding en nauwlettend volgen van het onderzoek een voortdurende steun is geweest. Dank ook aan Dr. A.K. Chesters, die gedurende de eerste jaren van het onderzoek een grote bron van inspiratie is geweest.

Waardering en dank voor de medewerkers en technici van het laboratorium voor Aero- en Hydrodynamica, die kosten noch moeite gespaard hebben on het project te doen slagen. Mijn bijzondere dank gaat uft naar Bert van der Velden voor de vele ontwerpen en technische adviezen en naar de afstudeerders Kees Borst en Arend Dijkshoorn die een belangrijke bijdrage hebben geleverd aan het verkrijgen van de resultaten.

Grote waardering ook voor het personeel van de Centrale Werkplaats, zonder hen zou het project niet mogelijk zijn geweest.

Dank tenslotte aan Birgitte Thomassen voor het typewerk en niet in de laatste plaats je steun.

Het project is mede tot stand gekomen met financiële steun van de Stichting voor de Technische Wetenschappen (STW). 


\section{Curriculum vitae}

\begin{tabular}{|c|c|}
\hline $26-02-1955$ & geboren te Neer. \\
\hline juni 1972 & eindexamen HBS-B te Roermond. \\
\hline $1972-1973$ & 1 jaar HTS natuurkunde te Eindhoven. \\
\hline $1973-1980$ & $\begin{array}{l}\text { studie aan de Technische Hogeschool te Elndhoven, } \\
\text { afd. natuurkunde, afstudeerhoogleraar prof. D.A. de Vries. }\end{array}$ \\
\hline $980-1981$ & $\begin{array}{l}\text { wetenschappelijk medewerker aan de Technische Hogeschool } \\
\text { Eindhoven, afdeling natuurkunde, vakgroep Transportfysica. }\end{array}$ \\
\hline $981-1986$ & wetenschappelijk medewerker aan de Technische Hogeschool \\
\hline
\end{tabular}

Sedert 1 juni 1986 werkzaam bij Océ-Nederland B.V. te Venlo. 